\title{
Ring-Opening of Epoxides by Pendant Silanols
}

\author{
Dr. Someshwar Nagamalla ${ }^{a}$, Professor Joel T. Mague ${ }^{b}$, and Professor Shyam Sathyamoorthi ${ }^{\mathrm{a} *}$ \\ aDepartment of Medicinal Chemistry, University of Kansas, Lawrence, KS, USA \\ ${ }^{b}$ Department of Chemistry, Tulane University, New Orleans, LA, USA
}

\section{Contents}

I. General considerations

II. Procedures for silanol epoxide substrate syntheses

III. Characterization of substrates in Schemes 3-5

IV. Procedures for ring-opening of epoxides

V. Characterization of products in Schemes 3-5

VI. Structural proof

VII. Crystal structure data for 41 (CCDC: 2126173)

VIII. Carbohydrate synthesis procedures and characterization

IX. $\quad{ }^{1} \mathrm{H}$ and ${ }^{13} \mathrm{C}$ NMR Spectra for all new compounds 


\section{General Considerations:}

All reagents were obtained commercially unless otherwise noted. Solvents were purified by passage under $10 \mathrm{psi} \mathrm{N}_{2}$ through activated alumina columns. Infrared (IR) spectra were recorded on a Thermo Scientific ${ }^{\mathrm{TM}}$ Nicolet ${ }^{\mathrm{TM}}$ iS ${ }^{\mathrm{TM}} 5 \mathrm{FT}$-IR Spectrometer; data are reported in frequency of absorption $\left(\mathrm{cm}^{-1}\right)$. NMR spectra were recorded on a Bruker Avance 400 operating at 400 and $100 \mathrm{MHz}$. ${ }^{1} \mathrm{H}$ NMR spectra were recorded at 400 $\mathrm{MHz}$. Data are recorded as: chemical shift in ppm referenced internally using residue solvent peaks, multiplicity $(\mathrm{s}=$ singlet, $\mathrm{d}=$ doublet, $\mathrm{t}=$ triplet, $\mathrm{q}=$ quartet, $\mathrm{m}=$ multiplet or overlap of nonequivalent resonances), integration, coupling constant ( $\mathrm{Hz}) .{ }^{13} \mathrm{C}$ NMR spectra were recorded at $100 \mathrm{MHz}$. Exact mass spectra were recorded using an electrospray ion source (ESI) either in positive mode or negative mode and with a time-of-flight (TOF) analyzer on a Waters LCT PremierTM mass spectrometer and are given in $\mathrm{m} / \mathrm{z}$. TLC was performed on pre-coated glass plates (Merck) and visualized either with a UV lamp ( $254 \mathrm{~nm}$ ) or by dipping into a solution of $\mathrm{KMnO}_{4}-\mathrm{K}_{2} \mathrm{CO}_{3}$ in water followed by heating. Flash chromatography was performed on silica gel (230-400 mesh) or Florisil (60-100 mesh).

\section{Procedures for Silanol Epoxide Substrate Syntheses}

\section{Procedure A}

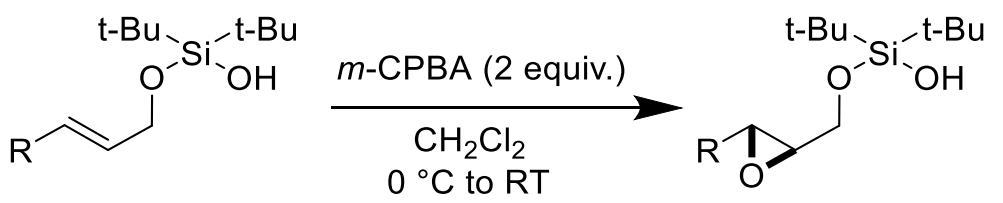

A $50 \mathrm{~mL}$ round-bottom flask was charged with a stir bar, alkenyl silanol substrate, and $\mathrm{CH}_{2} \mathrm{Cl}_{2}(0.1 \mathrm{M}$ final concentration). The homogenous mixture was cooled to $0{ }^{\circ} \mathrm{C}$ using an ice-water bath. $m$-CPBA (2 equivalents) was added in one portion, and the reaction flask was allowed to warm to room temperature over a period of two hours. Following this time, the reaction was quenched by careful addition of saturated aqueous $\mathrm{Na}_{2} \mathrm{~S}_{2} \mathrm{O}_{3}$ and transferred to a separatory funnel with $\mathrm{Et}_{2} \mathrm{O}$. The aqueous layer was removed, and the organic layer was washed with one portion of $0.5 \mathrm{M}$ aqueous $\mathrm{NaOH}$ solution. The organic layer was collected, dried with $\mathrm{MgSO}_{4}$, and concentrated under reduced pressure. The resulting residue was purified by chromatography (generally Florisil 60-100 Mesh but specific conditions are given for each compound).

\section{Procedure B}

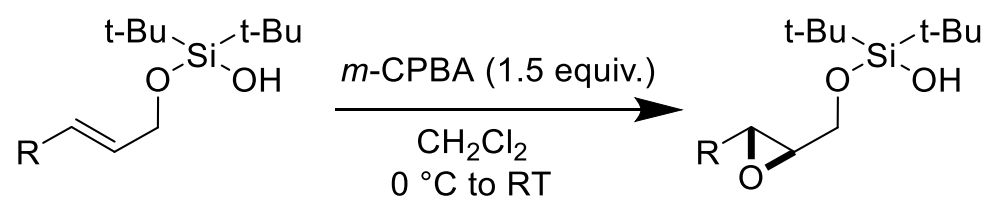

A $50 \mathrm{~mL}$ round-bottom flask was charged with a stir bar, alkenyl silanol substrate, and $\mathrm{CH}_{2} \mathrm{Cl}_{2}(0.1 \mathrm{M}$ final concentration). The homogenous mixture was cooled to $0{ }^{\circ} \mathrm{C}$ using an ice-water bath. $m$-CPBA (1.5 equivalents) was added in one portion, and the reaction flask was allowed to warm to room temperature over a period of two hours. Following this time, the reaction was quenched by careful addition of saturated aqueous $\mathrm{Na}_{2} \mathrm{~S}_{2} \mathrm{O}_{3}$ and transferred to a separatory funnel with $\mathrm{Et}_{2} \mathrm{O}$. The aqueous layer was removed, and the organic layer was washed with one portion of $0.5 \mathrm{M}$ aqueous $\mathrm{NaOH}$ solution. The organic layer was collected, dried with $\mathrm{MgSO}_{4}$, and concentrated under reduced pressure. 
The resulting residue was purified by chromatography (generally Florisil 60-100 Mesh but specific conditions are given for each compound).

\section{Procedure C}
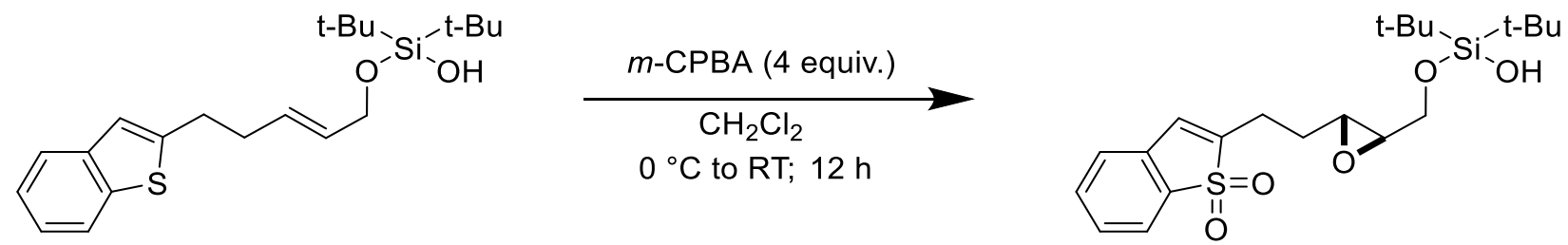

A $50 \mathrm{~mL}$ round-bottom flask was charged with a stir bar, alkenyl silanol substrate $(0.427 \mathrm{mmol}, 161 \mathrm{mg}$, 1 equiv.) and $\mathrm{CH}_{2} \mathrm{Cl}_{2}\left(4.3 \mathrm{~mL}, 0.1 \mathrm{M}\right.$ final concentration). The homogenous mixture was cooled to $0{ }^{\circ} \mathrm{C}$ using an ice-water bath. $m$-CPBA (1.71 mmol, $295 \mathrm{mg}$, 4 equivalents) was added in one portion, and the reaction flask was allowed to warm to room temperature over a period of twelve hours. Following this time, the reaction was quenched by careful addition of saturated aqueous $\mathrm{Na}_{2} \mathrm{~S}_{2} \mathrm{O}_{3}$ and transferred to a separatory funnel with $\mathrm{Et}_{2} \mathrm{O}$. The aqueous layer was removed, and the organic layer was washed with one portion of $0.5 \mathrm{M}$ aqueous $\mathrm{NaOH}$ solution. The organic layer was collected, dried with $\mathrm{MgSO}_{4}$, and concentrated under reduced pressure. The resulting residue was purified using a gradient of 0 to $50 \%$ EtOAc/hexanes on Florisil to yield product as a single diastereomer (white solid, $0.365 \mathrm{mmol}, 155 \mathrm{mg}$, $85 \%$ yield).

\section{Procedure D}
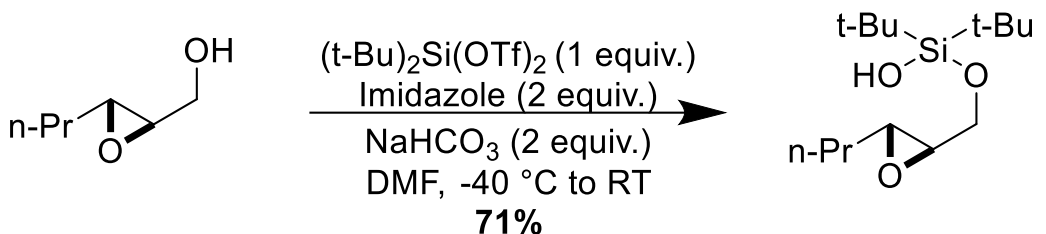

$71 \%$

A $5 \mathrm{~mL}$ microwave vial was charged with stir bar, imidazole ( $1 \mathrm{mmol}, 68 \mathrm{mg}, 2$ equivalents), and $4 \mathrm{~mL}$ of anhydrous DMF (99.8\% extra dry, AcroSeal). The reaction flask was cooled to $0{ }^{\circ} \mathrm{C}$ in an ice-water bath. $(\mathrm{t}-\mathrm{Bu})_{2} \mathrm{Si}(\mathrm{OTf})_{2}(0.5 \mathrm{mmol}, 0.164 \mathrm{~mL}, 1$ equivalent) was added dropwise. The reaction mixture was allowed to warm to room temperature over a period of 20 minutes. Following this time, the reaction mixture was cooled to $-40{ }^{\circ} \mathrm{C}$ using a dry ice/acetonitrile bath. $\mathrm{NaHCO}_{3}(1 \mathrm{mmol}, 84 \mathrm{mg}, 2$ equivalents) was added followed by epoxy-alcohol substrate $(0.5 \mathrm{mmol}, 58 \mathrm{mg}, 1$ equivalent $)$ in an additional $1 \mathrm{~mL}$ of DMF. The flask was sealed and allowed to warm to room temperature over a period of 4 hours. Following this time, the heterogenous mixture was transferred to a separatory funnel with $50 \mathrm{~mL}$ of diethyl ether. The organic layer was washed with $100 \mathrm{~mL}$ of $\mathrm{H}_{2} \mathrm{O}$, collected, dried with $\mathrm{MgSO}_{4}$, and concentrated under reduced pressure. The resulting residue was purified by chromatography on Florisil to yield silanol epoxide product ( $97 \mathrm{mg}, 0.355 \mathrm{mmol}, 71 \%$ yield). 


\section{Substrate Characterization Data}<smiles>CC(C)(C)[Si](O)(OCC1OC1P)C(C)(C)C</smiles>

di-tert-butyl(3-propyloxiran-2-yl) methoxy) silanol

Compound 1: Synthesized using Procedure A on a $1 \mathrm{mmol}$ scale; Purified using a gradient of 0 to $10 \%$ EtOAc/hexanes on Florisil; single diastereomer; (colorless oil, $241 \mathrm{mg}, 88 \%$ yield); ${ }^{1} \mathrm{H} \mathrm{NMR}(400 \mathrm{MHz}$, $\left.\mathrm{CDCl}_{3}\right) \delta 4.12(\mathrm{dd}, J=12.3,2.3 \mathrm{~Hz}, 1 \mathrm{H}), 3.73(\mathrm{~d}, J=5.7 \mathrm{~Hz}, 1 \mathrm{H}), 2.97-2.89(\mathrm{~m}, 2 \mathrm{H}), 1.65-1.41(\mathrm{~m}, 4 \mathrm{H})$, $1.03(\mathrm{~s}, 9 \mathrm{H}), 1.01(\mathrm{~s}, 9 \mathrm{H}), 0.96(\mathrm{t}, J=7.2 \mathrm{~Hz}, 3 \mathrm{H}) . ;{ }^{13} \mathrm{C}\left\{{ }^{1} \mathrm{H}\right\} \mathrm{NMR}\left(101 \mathrm{MHz}, \mathrm{CDCl}_{3}\right) \delta$ 64.2, 59.2, 57.0, 33.7, 27.5, 20.7, 19.4, 14.0.;IR: 3450, 2963, 2860, 1470, 1101, $650.9 \mathrm{~cm}^{-1}$.; HRMS (ESI) m/z: [M + Na+] calculated for $\mathrm{C}_{14} \mathrm{H}_{30} \mathrm{O}_{3} \mathrm{SiNa} 297.1862$, Found 297.1871.<smiles>CC(C)CC1OC1CO[Si](O)(C(C)(C)C)C(C)(C)C</smiles>

di-tert-butyl((3-isobutyloxiran-2-yl)methoxy)silanol

Compound 2: Synthesized using Procedure A on a $0.824 \mathrm{mmol}$ scale; Purified using a gradient of 0 to $10 \%$ EtOAc/hexanes on Florisil; single diastereomer; (colorless oil, $221 \mathrm{mg}, 93 \%$ yield); ${ }^{1} \mathrm{H}$ NMR (400 $\left.\mathrm{MHz}, \mathrm{CDCl}_{3}\right) \delta 4.15(\mathrm{dd}, J=12.3,2.6 \mathrm{~Hz}, 1 \mathrm{H}), 3.76(\mathrm{dd}, J=12.3,5.9 \mathrm{~Hz}, 1 \mathrm{H}), 3.01-2.94(\mathrm{~m}, 1 \mathrm{H}), 2.92(\mathrm{dt}, J$ $=5.9,2.5 \mathrm{~Hz}, 1 \mathrm{H}), 1.88-1.77(\mathrm{~m}, 1 \mathrm{H}), 1.56-1.47(\mathrm{~m}, 1 \mathrm{H}), 1.47-1.35(\mathrm{~m}, 1 \mathrm{H}), 1.05(\mathrm{~s}, 9 \mathrm{H}), 1.04(\mathrm{~s}, 9 \mathrm{H})$, $1.00(\mathrm{~d}, J=3.0 \mathrm{~Hz}, 3 \mathrm{H}), 0.98(\mathrm{~d}, J=3.0 \mathrm{~Hz}, 3 \mathrm{H}) .{ }^{13} \mathrm{C}\left\{{ }^{1} \mathrm{H}\right\} \mathrm{NMR}\left(101 \mathrm{MHz}, \mathrm{CDCl}_{3}\right) \delta$ 63.8, 59.2, 55.8, 40.6, 27.3, 26.3, 22.9, 22.3, 20.6, 20.5. IR 3442, 2951, 2866, 1461, 1361, $775 \mathrm{~cm}^{-1}$; HRMS (APCI) m/z: [M + $\left.\mathrm{H}^{+}\right]$ calculated for C15H33O3Si 289.2193, Found 289.2197.

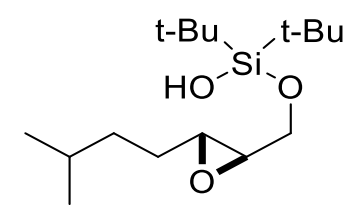

di-tert-butyl((3-isopentyloxiran-2-yl)methoxy)silanol

Compound 3: Synthesized using Procedure A on a $1 \mathrm{mmol}$ scale; Purified using a gradient of 0 to $10 \%$ EtOAc/Hexanes on Florisil; single diastereomer; (colorless oil, $287 \mathrm{mg}, 95 \%$ yield); ${ }^{1} \mathrm{H} \mathrm{NMR} \mathrm{(400} \mathrm{MHz}$, $\left.\mathrm{CDCl}_{3}\right) \delta 4.15(\mathrm{dd}, J=12.3,2.4 \mathrm{~Hz}, 1 \mathrm{H}), 3.79-3.69(\mathrm{~m}, 1 \mathrm{H}), 2.99-2.90(\mathrm{~m}, 2 \mathrm{H}), 1.68-1.54(\mathrm{~m}, 3 \mathrm{H}), 1.42$ - $1.28(\mathrm{~m}, 2 \mathrm{H}), 1.06(\mathrm{~s}, 9 \mathrm{H}), 1.04(\mathrm{~s}, 9 \mathrm{H}), 0.96-0.87(\mathrm{~m}, 6 \mathrm{H}) .{ }^{13} \mathrm{C}\left\{{ }^{1} \mathrm{H}\right\} \mathrm{NMR}\left(101 \mathrm{MHz}, \mathrm{CDCl}_{3}\right) \delta 64.0,59.1$, 57.2, 34.8, 29.4, 27.7, 27.3, 22.5, 22.4, 20.6, 20.5. IR 3437, 2946, 2866, 1473, 1387, 1113, $862 \mathrm{~cm}^{-1}$.; HRMS (ESI) m/z: [M + Na+] calculated for C16H34NaO3Si 325.2169 Found 325.2190. 


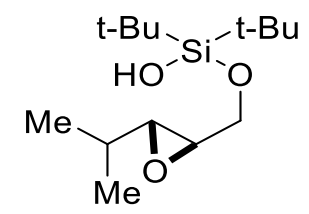

di-tert-butyl((3-isopropyloxiran-2-yl)methoxy)silanol

Compound 4: Synthesized using Procedure A on a $0.595 \mathrm{mmol}$ scale; Purified using a gradient of 0 to 20\% EtOAc/hexanes on Florisil; single diastereomer; (white semisolid, $153 \mathrm{mg}$, 94\% yield); ${ }^{1} \mathrm{H}$ NMR (400 $\left.\mathrm{MHz}, \mathrm{CDCl}_{3}\right) \delta 4.17-4.06(\mathrm{~m}, 1 \mathrm{H}), 3.74(\mathrm{dd}, J=12.3,6.1 \mathrm{~Hz}, 1 \mathrm{H}), 2.99(\mathrm{dt}, J=6.1,2.5 \mathrm{~Hz}, 1 \mathrm{H}$ ), $2.73(\mathrm{dd}, J$ $=6.9,2.4 \mathrm{~Hz}, 1 \mathrm{H}), 1.58(\mathrm{dd}, J=13.6,6.8 \mathrm{~Hz}, 1 \mathrm{H}), 1.04(\mathrm{~m}, 21 \mathrm{H}), 0.98(\mathrm{~d}, J=6.9 \mathrm{~Hz}, 3 \mathrm{H}) .{ }^{13} \mathrm{C}\left\{{ }^{1} \mathrm{H}\right\} \mathrm{NMR}(101$ $\left.\mathrm{MHz}, \mathrm{CDCl}_{3}\right) \delta 64.2,62.2,58.1,30.1,27.4,27.3,20.6,20.5,19.0,18.3$. IR 3448, 2968, 2866, 1473, 1101, $856 \mathrm{~cm}^{-1}$.; HRMS (ESI) m/z: [M + H+] calculated for C14H31O3Si 275.2037, Found 275.2031.

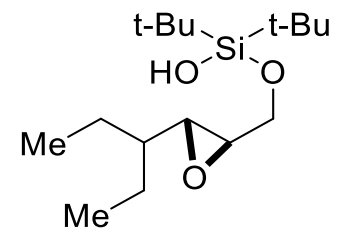

di-tert-butyl((3-(pentan-3-yl)oxiran-2-yl)methoxy)silanol

Compound 5: Synthesized using Procedure A on a $1.67 \mathrm{mmol}$ scale; Purified using a gradient of 0 to $20 \%$ EtOAc/Hexanes on silica gel; single diastereomer; (colorless oil, $440 \mathrm{mg}, 87 \%$ yield); ${ }^{1} \mathrm{H} \mathrm{NMR} \mathrm{(400} \mathrm{MHz,}$ $\left.\mathrm{CDCl}_{3}\right) \delta 4.16(\mathrm{dd}, J=12.3,2.6 \mathrm{~Hz}, 1 \mathrm{H}), 3.76(\mathrm{dd}, J=12.3,6.1 \mathrm{~Hz}, 1 \mathrm{H}), 2.97(\mathrm{dt}, J=6.1,2.5 \mathrm{~Hz}, 1 \mathrm{H}), 2.86(\mathrm{~s}$, $1 \mathrm{H}), 2.74(\mathrm{dd}, J=8.3,2.4 \mathrm{~Hz}, 1 \mathrm{H}), 1.61-1.42(\mathrm{~m}, 3 \mathrm{H}), 1.39-1.24(\mathrm{~m}, 1 \mathrm{H}), 1.15-1.07(\mathrm{~m}, 1 \mathrm{H}), 1.06(\mathrm{~s}$, 9H), $1.04(\mathrm{~s}, 9 \mathrm{H}), 1.00-0.96(\mathrm{~m}, 3 \mathrm{H}), 0.95-0.90(\mathrm{~m}, 3 \mathrm{H}) .{ }^{13} \mathrm{C}\left\{{ }^{1} \mathrm{H}\right\} \mathrm{NMR}\left(101 \mathrm{MHz}, \mathrm{CDCl}_{3}\right) \delta 64.1,60.5$, 58.6, 43.6, 27.3, 25.0, 23.5, 20.6, 20.5, 11.7, 10.9. IR 3442, 2968, 2860, 1473, 1101, $850 \mathrm{~cm}^{-1}$.; HRMS (ESI) $\mathrm{m} / \mathrm{z}:\left[\mathrm{M}+\mathrm{Na}^{+}\right]$calculated for $\mathrm{C} 16 \mathrm{H} 34 \mathrm{NaO} 3 \mathrm{Si} 325.2169$ Found 325.2148 .

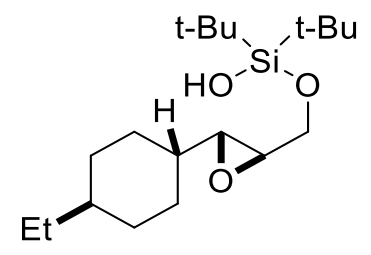

di-tert-butyl((3-((1r,4R)-4-ethylcyclohexyl)oxiran-2-yl)methoxy)silanol

Compound 6: Synthesized using Procedure A on a $0.823 \mathrm{mmol}$ scale; Purified using a gradient of 0 to $15 \%$ EtOAc/hexanes on Florisil; single diastereomer; (colorless oil, $217 \mathrm{mg}, 78 \%$ yield); ${ }^{1} \mathrm{H} \mathrm{NMR} \mathrm{(400}$ $\left.\mathrm{MHz}, \mathrm{CDCl}_{3}\right) \delta 4.14(\mathrm{dd}, J=12.3,2.6 \mathrm{~Hz}, 1 \mathrm{H}), 3.72(\mathrm{dd}, J=12.3,6.2 \mathrm{~Hz}, 1 \mathrm{H}), 3.01(\mathrm{dt}, J=6.2,2.5 \mathrm{~Hz}, 1 \mathrm{H})$, $2.73(\mathrm{dd}, J=6.7,2.4 \mathrm{~Hz}, 1 \mathrm{H}), 1.94-1.86(\mathrm{~m}, 1 \mathrm{H}), 1.86-1.78(\mathrm{~m}, 2 \mathrm{H}), 1.73(\mathrm{dd}, J=12.5,3.2 \mathrm{~Hz}, 1 \mathrm{H}), 1.30$ - $1.08(\mathrm{~m}, 6 \mathrm{H}), 1.06(\mathrm{~s}, 9 \mathrm{H}), 1.04(\mathrm{~s}, 9 \mathrm{H}), 0.96-0.81(\mathrm{~m}, 5 \mathrm{H}) .{ }^{13} \mathrm{C}\left\{{ }^{1} \mathrm{H}\right\} \mathrm{NMR}\left(101 \mathrm{MHz}, \mathrm{CDCl}_{3}\right) \delta 64.3,61.4$, 57.9, 39.8, 39.2, 32.0, 31.8, 29.8, 29.5, 28.8, 27.39, 27.36, 20.6, 20.5, 11.4. IR 3442, 1473, $1113,827 \mathrm{~cm}^{-}$ 1.; HRMS (ESI) m/z: [M + H ] calculated for C19H39O3Si 343.2663, Found 343.2663. 


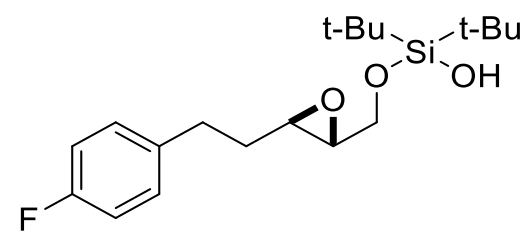

di-tert-butyl((3-(4-fluorophenethyl)oxiran-2-yl)methoxy)silanol

Compound 7: Synthesized using Procedure A on a $0.591 \mathrm{mmol} \mathrm{scale;} \mathrm{Purified} \mathrm{using} \mathrm{a} \mathrm{gradient} \mathrm{of} 0$ to $10 \% \mathrm{EtOAc} /$ hexanes on Florisil; single diastereomer; (colorless oil, $184 \mathrm{mg}, 88 \%$ yield); ${ }^{1} \mathrm{H}$ NMR (600 $\mathrm{MHz}, \mathrm{CDCl}_{3}$ ) $\delta 7.21-7.10(\mathrm{~m}, 2 \mathrm{H}), 7.05-6.97(\mathrm{~m}, 2 \mathrm{H}), 4.11$ (dd, $\left.J=12.4,2.5 \mathrm{~Hz}, 1 \mathrm{H}\right), 3.71$ (dd, $J=12.4$, $5.8 \mathrm{~Hz}, 1 \mathrm{H}$ ), 2.97 (ddd, $J=7.1,5.2,2.3 \mathrm{~Hz}, 1 \mathrm{H}$ ), 2.91 (dt, $J=5.7,2.4 \mathrm{~Hz}, 1 \mathrm{H}$ ), 2.82 (ddd, $J=14.3,8.5,6.1$ $\mathrm{Hz}, 1 \mathrm{H}), 2.73(\mathrm{dt}, J=14.0,8.1 \mathrm{~Hz}, 1 \mathrm{H}), 1.94-1.83(\mathrm{~m}, 2 \mathrm{H}), 1.05(\mathrm{~s}, 9 \mathrm{H}), 1.03(\mathrm{~s}, 9 \mathrm{H}) .{ }^{13} \mathrm{C}\left\{{ }^{1} \mathrm{H}\right\} \mathrm{NMR}(101$ $\left.\mathrm{MHz}, \mathrm{CDCl}_{3}\right) \delta 161.3(\mathrm{~d}, J=240 \mathrm{~Hz}$ ), 136.6, 129.7 (d, $J=7.8 \mathrm{~Hz}$ ), 115.2 (d, $J=21.1 \mathrm{~Hz}$ ), 63.6, 59.3, 55.9, 33.4, 31.4, 27.3, 20.6, 20.5. IR 3442, 2963, 2860, 1513, 1473, 1227, $850 \mathrm{~cm}^{-1}$.; HRMS (APCI) m/z: [M + H ] calculated for C19H32FO3Si 355.2099, Found 355.2105.<smiles>CC1([Mg])OC1CO[Si](O)(CC1CO1)C(C)(C)C</smiles>

3-(4-bromophenethyl) oxiran-2-yl)methoxy)di-tert-butylsilanol

Compound 8: Synthesized using Procedure A on a $1 \mathrm{mmol}$ scale; Purified using a gradient of 0 to $10 \%$ EtOAc/hexanes on Florisil; single diastereomer; (colorless oil, $365 \mathrm{mg}, 88 \%$ yield); ${ }^{1} \mathrm{H}$ NMR $(400 \mathrm{MHz}$, $\left.\mathrm{CDCl}_{3}\right) \delta 7.41(\mathrm{~d}, J=8.4 \mathrm{~Hz}, 2 \mathrm{H}), 7.07(\mathrm{~d}, J=8.4 \mathrm{~Hz}, 2 \mathrm{H}), 4.08(\mathrm{dd}, J=12.4,2.4 \mathrm{~Hz}, 1 \mathrm{H}), 3.69$ (dd, $J=12.4$, $5.8 \mathrm{~Hz}, 1 \mathrm{H}), 2.98-2.85(\mathrm{~m}, 2 \mathrm{H}), 2.79-2.64(\mathrm{~m}, 2 \mathrm{H}), 1.98-1.75(\mathrm{~m}, 2 \mathrm{H}), 1.02(\mathrm{~s}, 9 \mathrm{H}), 1.00(\mathrm{~s}, 9 \mathrm{H}) . ;{ }^{13} \mathrm{C}\left\{{ }^{1} \mathrm{H}\right\}$ $\operatorname{NMR}\left(101 \mathrm{MHz}, \mathrm{CDCl}_{3}\right) \delta$ 140.1, 131.7, 130.3, 120.0, 63.7, 59.4, 56.0, 33.3, 31.8, 27.5, 20.7, 20.6.; IR: 3440, 2932, 2860, 1473, 1136, $650.9 \mathrm{~cm}^{-1}$.; HRMS (ESI) m/z: [M + Na+] calculated for $\mathrm{C}_{19} \mathrm{H}_{31} \mathrm{BrO}_{3} \mathrm{SiNa} 437.1124$, Found 437.1117.<smiles>CC(C)(C)CC1OC1CO[Si](O)(CCCc1cccc(C(F)(F)F)c1)C(C)(C)C</smiles>

di-tert-butyl((3-(3-(trifluoromethyl)phenethyl)oxiran-2-yl)methoxy)silanol

Compound 9: Synthesized using Procedure A on a $0.806 \mathrm{mmol} \mathrm{scale;} \mathrm{Purified} \mathrm{using} \mathrm{a} \mathrm{gradient} \mathrm{of} 0$ to $10 \%$ EtOAc/hexanes on Florisil; single diastereomer; (colorless oil, $241 \mathrm{mg}, 74 \%$ yield); ${ }^{1} \mathrm{H}$ NMR (400 $\left.\mathrm{MHz}, \mathrm{CDCl}_{3}\right) \delta 7.58-7.38(\mathrm{~m}, 4 \mathrm{H}), 4.20-4.03(\mathrm{~m}, 1 \mathrm{H}), 3.73(\mathrm{dd}, J=12.4,5.7 \mathrm{~Hz}, 1 \mathrm{H}), 2.99$ (ddd, J = 6.9, 4.8, 2.3 Hz, 1H), $2.93(\mathrm{dt}, J=5.9,2.6 \mathrm{~Hz}, 1 \mathrm{H}), 2.91-2.77(\mathrm{~m}, 2 \mathrm{H}), 2.02-1.85(\mathrm{~m}, 2 \mathrm{H}), 1.05(\mathrm{~s}, 9 \mathrm{H}), 1.02$ (s, 9H). ${ }^{13} \mathrm{C}\left\{{ }^{1} \mathrm{H}\right\} \mathrm{NMR}\left(101 \mathrm{MHz}, \mathrm{CDCl}_{3}\right) \delta 141.9,131.8,130.8$ (q, J = 32.0 Hz), 128.9, $125.1-124.9(\mathrm{~m})$, $122.8(\mathrm{q}, J=270 \mathrm{~Hz}$ ), 123.1-122.9 (m), 63.4, 59.3, 55.7, 33.1, 32.0, 27.4, 27.3, 20.6, 20.5. IR 3442, 2934, 2860, 1473, 1164, $827 \mathrm{~cm}^{-1}$;; HRMS (ESI) m/z: [M + Na+] calculated for C2OH31F3NaO3Si 427.1887 Found 427.1901. 


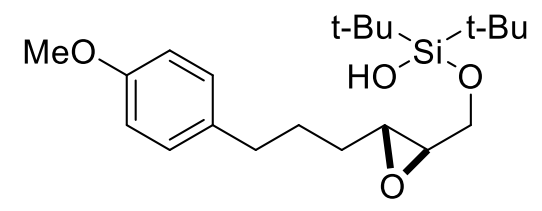

di-tert-butyl((3-(3-(4-methoxyphenyl)propyl)oxiran-2-yl)methoxy)silanol

Compound 10: Synthesized using Procedure A on a $0.851 \mathrm{mmol}$ scale; Purified using a gradient of 0 to $20 \%$ EtOAc/Hexanes on Florisil; single diastereomer; (colorless oil, $260 \mathrm{mg}, 80 \%$ yield); ${ }^{1} \mathrm{H}$ NMR (400 $\left.\mathrm{MHz}, \mathrm{CDCl}_{3}\right) \delta 7.17-7.06(\mathrm{~m}, 2 \mathrm{H}), 6.91-6.81(\mathrm{~m}, 2 \mathrm{H}), 4.20-4.10(\mathrm{~m}, 1 \mathrm{H}), 3.82(\mathrm{~s}, 3 \mathrm{H}), 3.73(\mathrm{dd}, J=$ $12.3,5.9 \mathrm{~Hz}, 1 \mathrm{H}), 3.02-2.88(\mathrm{~m}, 2 \mathrm{H}), 2.63(\mathrm{t}, J=7.5 \mathrm{~Hz}, 2 \mathrm{H}), 1.85-1.69(\mathrm{~m}, 2 \mathrm{H}), 1.69-1.52(\mathrm{~m}, 2 \mathrm{H})$, 1.06 (s, 9H), 1.04 (s, 9H). ${ }^{13} \mathrm{C}\left\{{ }^{1} \mathrm{H}\right\}$ NMR $\left(101 \mathrm{MHz}, \mathrm{CDCl}_{3}\right) \delta$ 157.8, 133.9, 129.2, 113.8, 63.8, 59.0, 56.7, 55.2, 34.5, 31.0, 27.9, 27.3, 20.6, 20.5. IR 3447, 2940, 2854, 1518, 1467, $1119 \mathrm{~cm}^{-1}$.; HRMS (ESI) m/z: [M $+\mathrm{Na}^{+}$] calculated for $\mathrm{C} 21 \mathrm{H} 36 \mathrm{NaO} 4 \mathrm{Si}$ 403.2275 Found 403.2289.

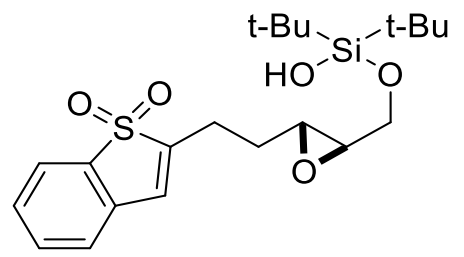

2-(2-(3-(((di-tert-butyl(hydroxy)silyl)oxy)methyl)oxiran-2-yl)ethyl)benzo[b]thiophene 1,1-dioxide

Compound 11: Synthesized using Procedure $\mathrm{C}$ on a $0.427 \mathrm{mmol}$ scale; Purified using a gradient of 0 to $50 \%$ EtOAc/hexanes on Florisil; single diastereomer; (white solid, $155 \mathrm{mg}, 85 \%$ yield); ${ }^{1} \mathrm{H} \mathrm{NMR} \mathrm{(400} \mathrm{MHz,}$ $\left.\mathrm{CDCl}_{3}\right) \delta 7.76-7.70(\mathrm{~m}, 1 \mathrm{H}), 7.57(\mathrm{td}, J=7.5,1.2 \mathrm{~Hz}, 1 \mathrm{H}), 7.49(\mathrm{td}, J=7.6,1.1 \mathrm{~Hz}, 1 \mathrm{H}), 7.33(\mathrm{~d}, J=7.4 \mathrm{~Hz}$, $1 \mathrm{H}), 6.88(\mathrm{q}, J=1.5 \mathrm{~Hz}, 1 \mathrm{H}$ ), 4.15 (dd, $J=12.4,2.6 \mathrm{~Hz}, 1 \mathrm{H}$ ), 3.79 (dd, $J=12.4,5.5 \mathrm{~Hz}, 1 \mathrm{H}$ ), 3.10 (ddd, $J=$ $6.8,4.2,2.3 \mathrm{~Hz}, 1 \mathrm{H}), 3.05(\mathrm{dt}, J=5.2,2.5 \mathrm{~Hz}, 1 \mathrm{H}), 2.87-2.72(\mathrm{~m}, 2 \mathrm{H}), 2.31-2.16(\mathrm{~m}, 1 \mathrm{H}), 1.98-1.82(\mathrm{~m}$, 1H), 1.05 (s, 9H), 1.03 (s, 9H). ${ }^{13} \mathrm{C}\left\{{ }^{1} \mathrm{H}\right\}$ NMR (101 MHz, CDCl $) \delta 143.7,136.7,133.7,131.3,129.6,126.2$, 124.5, 121.5, 63.3, 59.1, 55.4, 29.0, 27.5, 27.3, 21.1, 20.5. IR 3562, 2934, 2860, 1473, 1296, 1141, 827 $\mathrm{cm}^{-1}$.; HRMS (APCl) m/z: [M + $\left.\mathrm{H}^{+}\right]$calculated for C21H33O5SSi+ 425.1818, Found 425.1844.

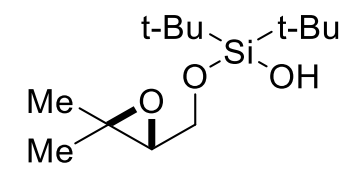

di-tert-butyl((3,3-dimethyloxiran-2-yl)methoxy)silanol

Compound 12: Synthesized using Procedure A on a $0.646 \mathrm{mmol}$ scale; Purified using a gradient of 0 to $10 \%$ EtOAc/hexanes on Florisil; (colorless oil, $152 \mathrm{mg}, 90 \%$ yield); $\left.{ }^{1} \mathrm{H} \mathrm{NMR} \mathrm{(400} \mathrm{MHz,} \mathrm{CDCl}_{3}\right) \delta 4.02$ (ddd, $J=11.9,4.0,1.8 \mathrm{~Hz}, 1 \mathrm{H}), 3.90-3.78(\mathrm{~m}, 1 \mathrm{H}), 3.03-2.95(\mathrm{~m}, 1 \mathrm{H}), 1.35(\mathrm{~d}, J=1.5 \mathrm{~Hz}, 3 \mathrm{H}), 1.32(\mathrm{~d}, J=1.5$ $\mathrm{Hz}, 3 \mathrm{H}), 1.03(\mathrm{~m}, 18 \mathrm{H}) .{ }^{13} \mathrm{C}\left\{{ }^{1} \mathrm{H}\right\} \mathrm{NMR}\left(101 \mathrm{MHz}, \mathrm{CDCl}_{3}\right) \delta 64.3,62.6,27.3,27.2,24.8,20.6,20.4,18.9$. IR 3460, 2968, 2854, 1467, 1393, 1084, $753 \mathrm{~cm}^{-1}$.; HRMS (APCI) m/z: $\left[\mathrm{M}+\mathrm{H}^{+}\right]$calculated for $\mathrm{C} 13 \mathrm{H} 2903 \mathrm{Si}$ 261.1886, Found 261.1906. 
<smiles>CCC1OC1CCO[Si](O)(C(C)(C)C)C(C)(C)C</smiles>

di-tert-butyl(2-(3-ethyloxiran-2-yl)ethoxy)silanol

Compound 13: Synthesized using Procedure A on a $1 \mathrm{mmol}$ scale; Purified using a gradient of 0 to $10 \%$ EtOAc/hexanes on Florisil; single diastereomer; (colorless oil, $246 \mathrm{mg}, 90 \%$ yield); ${ }^{1} \mathrm{H}$ NMR (400 MHz, $\left.\mathrm{CDCl}_{3}\right) \delta 3.99-3.90(\mathrm{~m}, 2 \mathrm{H}), 2.87$ (ddd, $\left.J=6.8,4.7,2.3 \mathrm{~Hz}, 1 \mathrm{H}\right), 2.73(\mathrm{td}, J=5.5,2.4 \mathrm{~Hz}, 1 \mathrm{H}), 1.91-1.78$ $(\mathrm{m}, 1 \mathrm{H}), 1.77-1.64(\mathrm{~m}, 1 \mathrm{H}), 1.63-1.50(\mathrm{~m}, 2 \mathrm{H}), 1.02(\mathrm{~s}, 9 \mathrm{H}), 1.02(\mathrm{~s}, 9 \mathrm{H}), 0.98(\mathrm{~d}, J=7.5 \mathrm{~Hz}, 3 \mathrm{H}) . ;{ }^{13} \mathrm{C}\left\{{ }^{1} \mathrm{H}\right\}$ $\operatorname{NMR}\left(101 \mathrm{MHz}, \mathrm{CDCl}_{3}\right) \delta$ 60.7, 60.3, 56.4, 35.5, 27.6, 27.6, 25.3, 20.6, 10.0.; IR: 3448, 2950, 2860, 1370, $1100,650.9 \mathrm{~cm}^{-1}$.; HRMS (ESI) m/z: [M + Na+] calculated for $\mathrm{C}_{14} \mathrm{H}_{30} \mathrm{O}_{3} \mathrm{SiNa} 297.1862$, Found 297.1840.

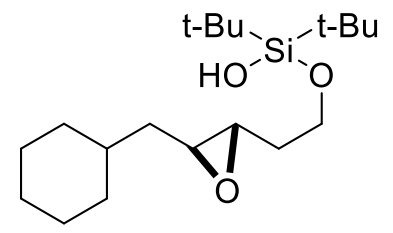

di-tert-butyl(2-(3-(cyclohexylmethyl)oxiran-2-yl)ethoxy)silanol

Compound 14: Synthesized using Procedure A on a $0.898 \mathrm{mmol}$ scale; Purified using reversed-phase HPLC (gradient of $100 \% \mathrm{H}_{2} \mathrm{O}$ with $0.1 \%$ TFA to $100 \% \mathrm{MeCN}$ with $0.1 \%$ TFA over 45 minutes on a Hamilton PRP-1.7 $\mu \mathrm{m}, 21.2 \times 250 \mathrm{~mm}, \mathrm{C} 18$ column); single diastereomer; (colorless oil, $168 \mathrm{mg}, 55 \%$ yield); ${ }^{1} \mathrm{H}$ $\operatorname{NMR}\left(400 \mathrm{MHz}, \mathrm{CDCl}_{3}\right) \delta 4.04-3.88(\mathrm{~m}, 2 \mathrm{H}), 2.91-2.75(\mathrm{~m}, 2 \mathrm{H}), 1.89$ (dddd, $J=14.3,7.3,5.9,4.5 \mathrm{~Hz}$, $1 \mathrm{H}), 1.85-1.63(\mathrm{~m}, 6 \mathrm{H}), 1.55-1.36(\mathrm{~m}, 3 \mathrm{H}), 1.35-1.14(\mathrm{~m}, 3 \mathrm{H}), 1.09-1.02(\mathrm{~m}, 18 \mathrm{H}), 1.02-0.93(\mathrm{~m}$, 2H). ${ }^{13} \mathrm{C}\left\{{ }^{1} \mathrm{H}\right\}$ NMR $\left(101 \mathrm{MHz}, \mathrm{CDCl}_{3}\right) \delta 60.5,58.0,57.0,40.0,35.8,35.4,33.8,33.2,27.6,27.5,26.5,26.4$, 26.3, 20.6. IR 3442, 2940, 2866, 1473, 1101, $827 \mathrm{~cm}^{-1}$; HRMS (ESI) m/z: [M + Na+] calculated for C19H38NaO3Si 365.2482 Found 365.2492.<smiles>CC[C@H]1O[C@@H]1CCO[Si](O)(C(C)(C)C)C(C)(C)C</smiles>

di-tert-butyl(2-(3-ethyloxiran-2-yl)ethoxy)silanol

Compound 15: Synthesized using Procedure A on a $1 \mathrm{mmol}$ scale; Purified using a gradient of 0 to $10 \%$ EtOAc/hexanes on Florisil; single diastereomer; (colorless oil, $232 \mathrm{mg}, 85 \%$ yield); ${ }^{1} \mathrm{H} \mathrm{NMR}$ (400 MHz, $\left.\mathrm{CDCl}_{3}\right) \delta 4.00$ (ddd, $J=6.2,5.4,2.3 \mathrm{~Hz}, 2 \mathrm{H}$ ), $3.13(\mathrm{dt}, J=7.7,4.5 \mathrm{~Hz}, 1 \mathrm{H}$ ), 2.93 (ddd, $J=6.8,6.0,4.3 \mathrm{~Hz}, 1 \mathrm{H}$ ), $2.26(\mathrm{~s}, 1 \mathrm{H}), 1.86$ (dddd, $J=13.9,7.4,6.3,4.6 \mathrm{~Hz}, 1 \mathrm{H}), 1.73-1.52(\mathrm{~m}, 3 \mathrm{H}), 1.07-1.03(\mathrm{~m}, 3 \mathrm{H}), 1.02(\mathrm{~s}$, $18 \mathrm{H}) . ;{ }^{13} \mathrm{C}\left\{{ }^{1} \mathrm{H}\right\} \mathrm{NMR}\left(101 \mathrm{MHz}, \mathrm{CDCl}_{3}\right) \delta 61.3,58.5,55.0,31.1,27.6,27.5,21.4,20.6,10.7 . ;$ IR: 3464, 2945, 2866, 1145, $650.9 \mathrm{~cm}^{-1}$.; HRMS (ESI) m/z: [M+ Na+] calculated for $\mathrm{C}_{14} \mathrm{H}_{30} \mathrm{O}_{3} \mathrm{SiNa} 297.1862$, Found 297.1871. 
<smiles>CC(C)(C)[Si](O)(CC1OC1P)OCC1OC1=P</smiles>

di-tert-butyl(3-propyloxiran-2-yl) methoxy) silanol

Compound 16: Synthesized using Procedure A on a $1 \mathrm{mmol}$ scale; Purified using a gradient of 0 to $10 \%$ EtOAc/hexanes on Florisil; single diastereomer; (colorless oil, $244 \mathrm{mg}, 88 \%$ yield); ${ }^{1} \mathrm{H}$ NMR (400 MHz, $\left.\mathrm{CDCl}_{3}\right) \delta 4.03(\mathrm{dd}, J=12.0,3.8 \mathrm{~Hz}, 1 \mathrm{H}$ ), $3.84(\mathrm{dd}, J=12.0,7.6 \mathrm{~Hz}, 1 \mathrm{H}$ ), 3.16 (ddd, $J=7.6,4.4,3.7 \mathrm{~Hz}, 1 \mathrm{H}$ ), $3.10-3.01(\mathrm{~m}, 1 \mathrm{H}), 1.58-1.47(\mathrm{~m}, 4 \mathrm{H}), 1.03(\mathrm{~s}, 9 \mathrm{H}), 1.03(\mathrm{~s}, 9 \mathrm{H}), 0.99-0.93(\mathrm{~m}, 3 \mathrm{H}) . ;{ }^{13} \mathrm{C}\left\{{ }^{1} \mathrm{H}\right\} \mathrm{NMR}(101$ $\left.\mathrm{MHz}, \mathrm{CDCl}_{3}\right) \delta 62.2,57.9,57.5,30.2,27.5,27.4,20.8,20.6,20.1,14.0$.; IR: 3450, 2936, 2870, 1140, 650.9 $\mathrm{cm}^{-1}$; HRMS (ESI) m/z: [M + Na+] calculated for $\mathrm{C}_{14} \mathrm{H}_{30} \mathrm{O}_{3} \mathrm{SiNa} 297.1862$, Found 297.1867.

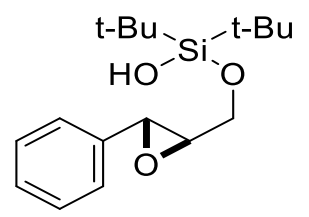

di-tert-butyl(3-phenyloxiran-2-yl)methoxy)silanol

Compound 17: Synthesized using Procedure B on a $0.513 \mathrm{mmol} \mathrm{scale;} \mathrm{Single} \mathrm{diastereomer;} \mathrm{Purified} \mathrm{using}$ a gradient of 0 to $20 \%$ EtOAc/hexanes on Florisil; (colorless oil, $128 \mathrm{mg}, 81 \%$ yield); ${ }^{1} \mathrm{H}$ NMR (400 MHz, $\left.\mathrm{CDCl}_{3}\right) \delta 7.45-7.21(\mathrm{~m}, 5 \mathrm{H}), 4.29(\mathrm{dd}, J=12.4,2.5 \mathrm{~Hz}, 1 \mathrm{H}), 3.99-3.89(\mathrm{~m}, 2 \mathrm{H}), 3.24(\mathrm{dt}, J=4.9,2.4 \mathrm{~Hz}$, 1H), 1.09 (s, 9H), 1.07 (s, 9H). ${ }^{13} \mathrm{C}\left\{{ }^{1} \mathrm{H}\right\} \operatorname{NMR}\left(101 \mathrm{MHz}, \mathrm{CDCl}_{3}\right) \delta$ 136.7, 128.5, 128.3, 125.7, 63.2, 62.9, 56.3, 27.4, 27.3, 20.6, 20.5. IR 3448, 2940, 2860, 1467, 1107, $827 \mathrm{~cm}^{-1}$.; HRMS (ESI) m/z: [M + $\left.\mathrm{Na}^{+}\right]$calculated for $\mathrm{C} 17 \mathrm{H} 28 \mathrm{NaO} 3 \mathrm{Si} 331.1700$ Found 331.1713.

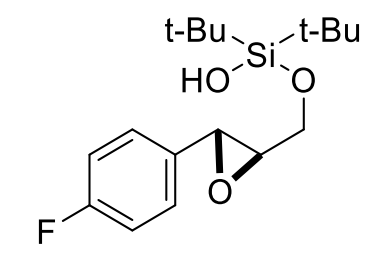

di-tert-butyl((3-(4-fluorophenyl)oxiran-2-yl)methoxy)silanol

Compound 18: Synthesized using Procedure B on a $1 \mathrm{mmol}$ scale; Purified using a gradient of 0 to $10 \%$ EtOAc/Hexanes on Florisil; single diastereomer; (colorless oil, $218 \mathrm{mg}, 67 \%$ yield); ${ }^{1} \mathrm{H} \mathrm{NMR} \mathrm{(600} \mathrm{MHz,}$ $\left.\mathrm{CDCl}_{3}\right) \delta 7.30-7.23(\mathrm{~m}, 2 \mathrm{H}), 7.09-7.03(\mathrm{~m}, 2 \mathrm{H}), 4.27(\mathrm{dd}, J=12.3,2.5 \mathrm{~Hz}, 1 \mathrm{H}), 3.96(\mathrm{dd}, J=12.3,5.0 \mathrm{~Hz}$, $1 \mathrm{H}), 3.91(\mathrm{~d}, J=2.1 \mathrm{~Hz}, 1 \mathrm{H}), 3.20(\mathrm{dt}, J=4.8,2.3 \mathrm{~Hz}, 1 \mathrm{H}), 1.08(\mathrm{~s}, 9 \mathrm{H}), 1.07(\mathrm{~s}, 9 \mathrm{H}) .{ }^{13} \mathrm{C}\left\{{ }^{1} \mathrm{H}\right\} \mathrm{NMR}(101 \mathrm{MHz}$, $\left.\mathrm{CDCl}_{3}\right) \delta 162.7(\mathrm{~d}, J=246.6 \mathrm{~Hz}$ ), 132.5 (d, $J=3.0 \mathrm{~Hz}), 127.4(\mathrm{~d}, J=8.3 \mathrm{~Hz}), 115.5(\mathrm{~d}, J=21.7 \mathrm{~Hz}), 63.0$, 62.9, 55.7, 27.4, 27.3, 20.6, 20.5. IR 3449, 2934, 2860, 1513, $1113 \mathrm{~cm}^{-1}$.; HRMS (ESI) m/z: [M + $\left.\mathrm{Na}^{+}\right]$ calculated for C17H27FNaO3Si 349.1606 Found 349.1634. 


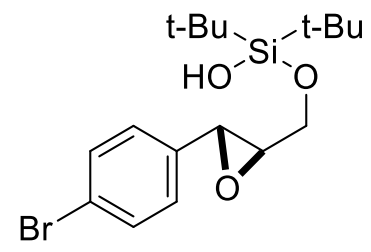

((3-(4-bromophenyl)oxiran-2-yl)methoxy)di-tert-butylsilanol

Compound 19: Synthesized using Procedure B on a $0.445 \mathrm{mmol}$ scale; Purified using a gradient of 0 to

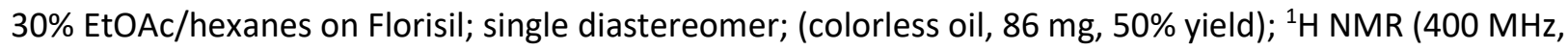
$\left.\mathrm{CDCl}_{3}\right) \delta 7.52-7.48(\mathrm{~m}, 2 \mathrm{H}), 7.20-7.15(\mathrm{~m}, 2 \mathrm{H}), 4.26(\mathrm{dd}, J=12.3,2.6 \mathrm{~Hz}, 1 \mathrm{H}), 3.96$ (dd, $J=12.4,4.9 \mathrm{~Hz}$, $1 \mathrm{H}), 3.88(\mathrm{~d}, J=2.1 \mathrm{~Hz}, 1 \mathrm{H}), 3.18(\mathrm{dt}, J=4.8,2.4 \mathrm{~Hz}, 1 \mathrm{H}), 1.08(\mathrm{~s}, 9 \mathrm{H}), 1.06(\mathrm{~s}, 9 \mathrm{H}) .{ }^{13} \mathrm{C}\left\{{ }^{1} \mathrm{H}\right\} \mathrm{NMR}(101 \mathrm{MHz}$, $\left.\mathrm{CDCl}_{3}\right) \delta 135.9,131.6,127.3,122.1,63.0,62.9,55.6,27.4,27.3,20.6,20.5$. IR 3442, 1934, 2860, 1473, $1113 \mathrm{~cm}^{-1}$; HRMS (ESI) m/z: [M + Na+] calculated for C17H27BrNaO3Si 409.0805 Found 409.0824.<smiles>CC(C)(C)[Si](O)(OCC1OC1c1ccc(Cl)cc1)C(C)(C)C</smiles>

di-tert-butyl((3-(4-chlorophenyl)oxiran-2-yl)methoxy)silanol

Compound 20: Synthesized using Procedure B on a $1.19 \mathrm{mmol} \mathrm{scale}$; Purified using a gradient of 0 to $10 \% \mathrm{EtOAc} /$ hexanes on Florisil; single diastereomer; (colorless oil, $347 \mathrm{mg}, 85 \%$ yield); ${ }^{1} \mathrm{H}$ NMR (400 $\left.\mathrm{MHz}, \mathrm{CDCl}_{3}\right) \delta 7.39-7.31(\mathrm{~m}, 2 \mathrm{H}), 7.28-7.10(\mathrm{~m}, 2 \mathrm{H}), 4.26(\mathrm{dd}, J=12.3,2.6 \mathrm{~Hz}, 1 \mathrm{H}), 3.96(\mathrm{dd}, J=12.3$, $4.9 \mathrm{~Hz}, 1 \mathrm{H}), 3.90(\mathrm{~d}, J=2.1 \mathrm{~Hz}, 1 \mathrm{H}), 3.18(\mathrm{dt}, J=4.8,2.4 \mathrm{~Hz}, 1 \mathrm{H}), 1.08(\mathrm{~s}, 9 \mathrm{H}), 1.06(\mathrm{~s}, 9 \mathrm{H}) .{ }^{13} \mathrm{C}\left\{{ }^{1} \mathrm{H}\right\} \mathrm{NMR}$ $\left(101 \mathrm{MHz}, \mathrm{CDCl}_{3}\right) \delta 135.3,134.0,128.7,127.0,63.0,62.9,55.6,27.4,27.3,20.6,20.5$. IR 3448, 2934, 2860, 1473, $1090 \mathrm{~cm}^{-1}$; HRMS (ESI) m/z: [M + Na+] calculated for C17H27CINaO3Si 365.1310 Found 365.1346 .<smiles>CC(C)(C)[Si](C)(C)O[Si](OCC1OC1c1cccc(Br)c1)(C(C)(C)C)C(C)(C)C</smiles>

((3-(3-bromophenyl)oxiran-2-yl)methoxy)di-tert-butylsilanol

Compound 21: Synthesized using Procedure B on a $0.846 \mathrm{mmol}$ scale; Purified using a gradient of 0 to $20 \%$ EtOAc/Hexanes on Florisil; single diastereomer; (colorless oil, $310 \mathrm{mg}, 95 \%$ yield); ${ }^{1} \mathrm{H}$ NMR (400 $\left.\mathrm{MHz}, \mathrm{CDCl}_{3}\right) \delta 7.51-7.40(\mathrm{~m}, 2 \mathrm{H}), 7.26-7.13(\mathrm{~m}, 2 \mathrm{H}), 4.26(\mathrm{dd}, J=12.4,2.6 \mathrm{~Hz}, 1 \mathrm{H}), 3.96(\mathrm{dd}, J=12.4$, $4.8 \mathrm{~Hz}, 1 \mathrm{H}), 3.89(\mathrm{~d}, J=2.1 \mathrm{~Hz}, 1 \mathrm{H}), 3.18(\mathrm{dt}, J=4.7,2.4 \mathrm{~Hz}, 1 \mathrm{H}), 1.08(\mathrm{~s}, 9 \mathrm{H}), 1.06(\mathrm{~s}, 9 \mathrm{H}) .{ }^{13} \mathrm{C}\left\{{ }^{1} \mathrm{H}\right\} \mathrm{NMR}$ $\left(101 \mathrm{MHz}, \mathrm{CDCl}_{3}\right) \delta 139.3,131.3,130.0,128.6,124.3,122.7,63.1,62.7,55.3,27.4,27.3,20.6,20.5$. IR 3460, 2951, 1467, 1147, $845 \mathrm{~cm}^{-1}$; HRMS (ESI) m/z: [M + Na+] calculated for C17H27BrNaO3Si 409.0805, Found 409.0799. 


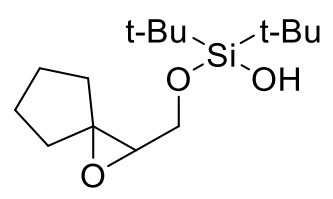

((1-oxaspiro[2.4]heptan-2-yl)methoxy)di-tert-butylsilanol

Compound 22: Synthesized using Procedure A on a $1 \mathrm{mmol}$ scale; Purified using a gradient of 0 to $10 \%$ EtOAc/Hexanes on Florisil; (colorless oil, $283 \mathrm{mg}$, 98\% yield); $\left.{ }^{1} \mathrm{H} \mathrm{NMR} \mathrm{(400} \mathrm{MHz,} \mathrm{CDCl}_{3}\right) \delta 4.05$ (ddd, $\mathrm{J}=$ 12.0, 3.5, $1.5 \mathrm{~Hz}, 1 \mathrm{H}$ ), 3.77 (dd, $J=12.0,7.7 \mathrm{~Hz}, 1 \mathrm{H}$ ), 3.24 (dd, $J=7.7,3.5 \mathrm{~Hz}, 1 \mathrm{H}), 2.00-1.75(\mathrm{~m}, 4 \mathrm{H})$, 1.67 (dddt, $J=12.5,9.4,8.2,6.0 \mathrm{~Hz}, 4 \mathrm{H}), 1.05(\mathrm{~d}, J=0.8 \mathrm{~Hz}, 9 \mathrm{H}), 1.04(\mathrm{~d}, J=0.8 \mathrm{~Hz}, 9 \mathrm{H}) .{ }^{13} \mathrm{C}\left\{{ }^{1} \mathrm{H}\right\} \mathrm{NMR}$ $\left(101 \mathrm{MHz}, \mathrm{CDCl}_{3}\right) \delta 70.1,63.9,61.8,33.7,29.2,27.3,27.2,25.1,24.7,20.6,20.4$. IR 3448, 2946, 2860, 1467, $1136 \mathrm{~cm}^{-1}$.; HRMS (ESI) m/z: [M + Na+] calculated for C15H3ONaO3Si 309.1856 Found 309.1867.

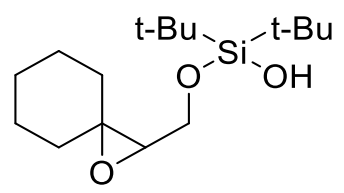

((1-oxaspiro[2.5]octan-2-yl)methoxy)di-tert-butylsilanol

Compound 23: Synthesized using Procedure A on a $1.26 \mathrm{mmol}$ scale; Purified using a gradient of 0 to $10 \%$ EtOAc/hexanes on Florisil; (colorless oil, $361 \mathrm{mg}, 95 \%$ yield); ${ }^{1} \mathrm{H}$ NMR $\left(400 \mathrm{MHz}, \mathrm{CDCl}_{3}\right) \delta 4.04$ (ddd, $J=11.9,4.2,1.9 \mathrm{~Hz}, 1 \mathrm{H}), 3.90(\mathrm{dd}, J=11.9,7.3 \mathrm{~Hz}, 1 \mathrm{H}), 2.98(\mathrm{ddd}, J=7.4,4.1,1.0 \mathrm{~Hz}, 1 \mathrm{H}), 1.81-1.65(\mathrm{~m}$, 2H), $1.64-1.44(\mathrm{~m}, 8 \mathrm{H}), 1.05$ (s, 9H), $1.04(\mathrm{~s}, 9 \mathrm{H}) .{ }^{13} \mathrm{C} \mathrm{NMR}\left(101 \mathrm{MHz}, \mathrm{CDCl}_{3}\right) \delta$ 64.6, 64.0, 62.1, 35.4, 29.6, 27.4, 27.2, 25.5, 24.9, 24.8, 20.6, 20.4. IR 3442, 2963, 2854, 1478, 1130, 1010, $839 \mathrm{~cm}^{-1}$.; HRMS (ESI) $\mathrm{m} / \mathrm{z}:\left[\mathrm{M}+\mathrm{Na}^{+}\right]$calculated for $\mathrm{C} 16 \mathrm{H} 32 \mathrm{NaO} 3 \mathrm{Si} 323.2013$, Found 323.2026.

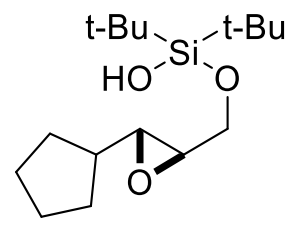

di-tert-butyl((3-cyclopentyloxiran-2-yl)methoxy)silanol

Compound 24: Synthesized using Procedure A on a $1.10 \mathrm{mmol}$ scale; Purified using a gradient of 0 to $20 \% \mathrm{EtOAc/hexanes} \mathrm{on} \mathrm{Florisil;} \mathrm{single} \mathrm{diastereomer;} \mathrm{(colorless} \mathrm{oil,} 264 \mathrm{mg}, 80 \%$ yield); ${ }^{1} \mathrm{H}$ NMR (400 $\left.\mathrm{MHz}, \mathrm{CDCl}_{3}\right) \delta 4.17-4.09(\mathrm{~m}, 1 \mathrm{H}), 3.74(\mathrm{dd}, J=12.2,6.0 \mathrm{~Hz}, 1 \mathrm{H}), 2.98(\mathrm{dt}, J=6.1,2.5 \mathrm{~Hz}, 1 \mathrm{H}), 2.86(\mathrm{dd}, J$ $=6.5,2.4 \mathrm{~Hz}, 1 \mathrm{H}), 1.89(\mathrm{dt}, J=14.7,7.5 \mathrm{~Hz}, 1 \mathrm{H}), 1.79(\mathrm{tdd}, J=12.0,6.2,2.8 \mathrm{~Hz}, 2 \mathrm{H}), 1.70-1.52(\mathrm{~m}, 4 \mathrm{H})$, $1.49-1.30(\mathrm{~m}, 2 \mathrm{H}), 1.05(\mathrm{~s}, 9 \mathrm{H}), 1.04(\mathrm{~s}, 9 \mathrm{H}) .{ }^{13} \mathrm{C}\left\{{ }^{1} \mathrm{H}\right\}$ NMR $\left(101 \mathrm{MHz}, \mathrm{CDCl}_{3}\right) \delta 64.1,60.3,58.5,40.9,29.1$, 28.7, 27.4, 27.3, 25.3, 20.6, 20.5. IR 3431, 2963, 2866, 1473, $1113 \mathrm{~cm}^{-1}$.; HRMS (ESI) m/z: [M + Na+] calculated for $\mathrm{C} 16 \mathrm{H} 32 \mathrm{NaO} 3 \mathrm{Si} 323.2013$ Found 323.2020. 
<smiles>CC(C)O[Si](C)(O)O[Si](C)(C)C</smiles>

di-tert-butyl(3-(3-methyloxiran-2-yl)propoxy)silanol

Compound 49: Synthesized using Procedure A on a $1 \mathrm{mmol}$ scale; Purified using a gradient of 0 to $10 \%$ EtOAc/hexanes on Florisil; single diastereomer; (colorless oil, $232 \mathrm{mg}, 85 \%$ yield); ${ }^{1} \mathrm{H}$ NMR (400 MHz, $\left.\mathrm{CDCl}_{3}\right) \delta 3.91-3.82(\mathrm{~m}, 2 \mathrm{H}), 2.78(\mathrm{dd}, J=5.2,2.3 \mathrm{~Hz}, 1 \mathrm{H}), 2.68(\mathrm{ddd}, J=6.6,3.9,2.3 \mathrm{~Hz}, 1 \mathrm{H}), 2.60(\mathrm{~s}, 1 \mathrm{H})$, $1.79-1.64(\mathrm{~m}, 3 \mathrm{H}), 1.62-1.51(\mathrm{~m}, 1 \mathrm{H}), 1.30(\mathrm{~d}, J=5.2 \mathrm{~Hz}, 3 \mathrm{H}), 1.02(\mathrm{~s}, 9 \mathrm{H}), 1.00(\mathrm{~s}, 9 \mathrm{H}) . ;{ }^{13} \mathrm{C}\left\{{ }^{1} \mathrm{H}\right\} \mathrm{NMR}$ (101 MHz, $\left.\mathrm{CDCl}_{3}\right) \delta$ 62.7, 60.3, 54.6, 29.8, 28.2, 27.7, 27.6, 20.8, 20.6, 17.7.; IR: 3437, 2933, 2860, 1473, 1130, $650.9 \mathrm{~cm}^{-1}$.; HRMS (ESI) m/z: [M + Na+] calculated for $\mathrm{C}_{14} \mathrm{H}_{30} \mathrm{O}_{3} \mathrm{SiNa} 297.1862$, Found 297.1871.<smiles>C[M][Si](O)(OCCC1O[C@H]1C(C)(C)C)O[Si](C)(C)C</smiles>

di-tert-butyl(3-(3-methyloxiran-2-yl)propoxy)silanol

Compound 51: Synthesized using Procedure A on a $1 \mathrm{mmol}$ scale; Purified using a gradient of 0 to $10 \%$ EtOAc/hexanes on Florisil; single diastereomer; (colorless oil, $242 \mathrm{mg}, 88 \%$ yield); ${ }^{1} \mathrm{H}$ NMR (400 MHz, $\left.\mathrm{CDCl}_{3}\right) \delta 3.94-3.85(\mathrm{~m}, 2 \mathrm{H}), 3.10-3.03(\mathrm{~m}, 1 \mathrm{H}), 2.98-2.89(\mathrm{~m}, 1 \mathrm{H}), 2.54(\mathrm{~d}, J=8.9 \mathrm{~Hz}, 1 \mathrm{H}), 1.79-1.60$ $(\mathrm{m}, 4 \mathrm{H}), 1.28(\mathrm{~d}, J=5.4 \mathrm{~Hz}, 3 \mathrm{H}), 1.03(\mathrm{~s}, 9 \mathrm{H}), 1.01(\mathrm{~s}, 9 \mathrm{H}) . ;{ }^{13} \mathrm{C}\left\{{ }^{1} \mathrm{H}\right\} \mathrm{NMR}\left(101 \mathrm{MHz}, \mathrm{CDCl}_{3}\right) \delta$ 62.6, 57.5, 52.8, 30.2, 27.7, 27.6, 23.9, 20.8, 20.5, 13.2.; IR: 3426, 2933, 2875, 1145, $650.9 \mathrm{~cm}^{-1}$;; HRMS (ESI) m/z: [M + Na+] calculated for $\mathrm{C}_{14} \mathrm{H}_{30} \mathrm{O}_{3} \mathrm{SiNa}^{+}$297.1862, Found 297.1875.

\section{Cyclization Procedures}

Procedure A
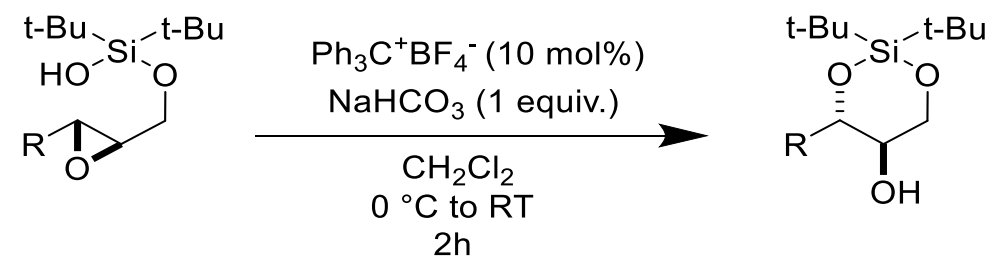

A $5 \mathrm{~mL}$ microwave vial was charged with a stir bar, silanol epoxide substrate $(0.2 \mathrm{mmol}), \mathrm{NaHCO}_{3}(0.2$ mmol, $16.8 \mathrm{mg}, 1$ equivalent) and anhydrous $\mathrm{CH}_{2} \mathrm{Cl}_{2}(1 \mathrm{~mL})$. The heterogeneous mixture was cooled to 0 ${ }^{\circ} \mathrm{C}$ using an ice-water bath. $\mathrm{Ph}_{3} \mathrm{C}^{+} \mathrm{BF}_{4}{ }^{-}(0.02 \mathrm{mmol}, 6.6 \mathrm{mg}, 0.1$ equivalents) was added in one portion, and the sides of the reaction flask were rinsed with an additional $1 \mathrm{~mL}$ of $\mathrm{CH}_{2} \mathrm{Cl}_{2}$ (Final concentration: 0.1 $\mathrm{M})$. The reaction mixture was allowed to warm to room temperature over a period of two hours, diluted with $\mathrm{CH}_{2} \mathrm{Cl}_{2}$, and transferred to a separatory funnel. The organic layer was washed with saturated aqueous $\mathrm{NaHCO}_{3}$, collected, dried with $\mathrm{MgSO}_{4}$, and concentrated under reduced pressure. The resulting 
residue was purified by chromatography on silica gel (specific conditions are associated with each compound).

\section{Procedure B}

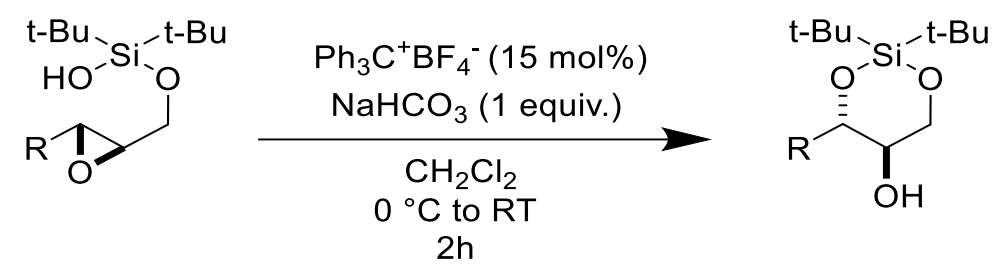

A $5 \mathrm{~mL}$ microwave vial was charged with a stir bar, silanol epoxide substrate $(0.2 \mathrm{mmol}), \mathrm{NaHCO}_{3}(0.2$ mmol, $16.8 \mathrm{mg}, 1$ equivalent) and anhydrous $\mathrm{CH}_{2} \mathrm{Cl}_{2}(1 \mathrm{~mL})$. The heterogeneous mixture was cooled to 0 ${ }^{\circ} \mathrm{C}$ using an ice-water bath. $\mathrm{Ph}_{3} \mathrm{C}^{+} \mathrm{BF}_{4}{ }^{-}(0.03 \mathrm{mmol}, 10 \mathrm{mg}, 0.15$ equivalents) was added in one portion, and the sides of the reaction flask were rinsed with an additional $1 \mathrm{~mL}$ of $\mathrm{CH}_{2} \mathrm{Cl}_{2}$ (Final concentration: 0.1 $\mathrm{M})$. The reaction mixture was allowed to warm to room temperature over a period of two hours, diluted with $\mathrm{CH}_{2} \mathrm{Cl}_{2}$, and transferred to a separatory funnel. The organic layer was washed with saturated aqueous $\mathrm{NaHCO}_{3}$, collected, dried with $\mathrm{MgSO}_{4}$, and concentrated under reduced pressure. The resulting residue was purified by chromatography on silica gel (specific conditions are associated with each compound).

Note: For a $1 \mathrm{mmol}$ scale reaction, the amounts are as follows: silanol epoxide substrate $(1.0 \mathrm{mmol})$, $\mathrm{NaHCO}_{3}$ (1 mmol, $84 \mathrm{mg}, 1$ equivalent), anhydrous $\mathrm{CH}_{2} \mathrm{Cl}_{2}(5 \mathrm{~mL}), \mathrm{Ph}_{3} \mathrm{C}^{+} \mathrm{BF}_{4}{ }^{-}(0.15 \mathrm{mmol}, 50 \mathrm{mg}, 0.15$ equivalents). All other parts of the procedure are identical.

\section{Procedure C}

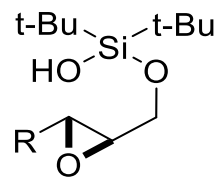

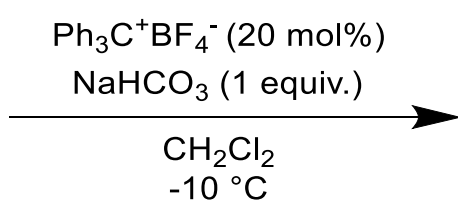

$2 \mathrm{~h}$

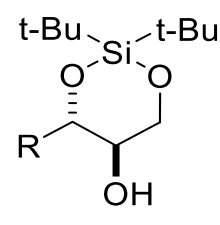

A $5 \mathrm{~mL}$ microwave vial was charged with a stir bar, silanol epoxide substrate $(0.2 \mathrm{mmol}), \mathrm{NaHCO}_{3}(0.2$ $\mathrm{mmol}, 16.8 \mathrm{mg}$, 1 equivalent) and anhydrous $\mathrm{CH}_{2} \mathrm{Cl}_{2}(1 \mathrm{~mL})$. The heterogeneous mixture was cooled to $-10{ }^{\circ} \mathrm{C}$ using a cryogenic cooler. $\mathrm{Ph}_{3} \mathrm{C}^{+} \mathrm{BF}_{4}{ }^{-}(0.04 \mathrm{mmol}, 13 \mathrm{mg}, 0.2$ equivalents) was added in one portion, and the sides of the reaction flask were rinsed with an additional $1 \mathrm{~mL}$ of $\mathrm{CH}_{2} \mathrm{Cl}_{2}$ (Final concentration: 0.1 $\mathrm{M})$. The reaction mixture was allowed to warm to room temperature over a period of two hours, diluted with $\mathrm{CH}_{2} \mathrm{Cl}_{2}$, and transferred to a separatory funnel. The organic layer was washed with saturated aqueous $\mathrm{NaHCO}_{3}$, collected, dried with $\mathrm{MgSO}_{4}$, and concentrated under reduced pressure. The resulting residue was purified by chromatography on silica gel (specific conditions are associated with each compound).

\section{$\underline{\text { Procedure D }}$}



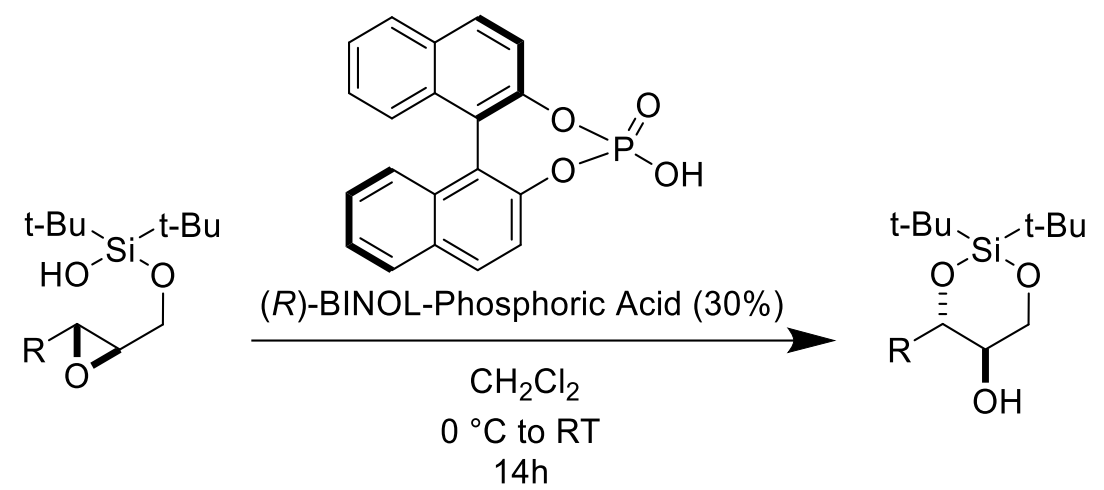

A $5 \mathrm{~mL}$ microwave vial was charged with a stir bar, silanol epoxide substrate $(0.2 \mathrm{mmol})$, and anhydrous $\mathrm{CH}_{2} \mathrm{Cl}_{2}(1 \mathrm{~mL})$. The homogenous mixture was cooled to $0{ }^{\circ} \mathrm{C}$ using an ice-water bath. $(R)$-BINOL-

Phosphoric acid (arbitrarily chosen) $(0.06 \mathrm{mmol}, 21 \mathrm{mg}, 0.3$ equivalents) was added in one portion, and the sides of the reaction flask were rinsed with an additional $1 \mathrm{~mL}$ of $\mathrm{CH}_{2} \mathrm{Cl}_{2}$ (Final concentration: $0.1 \mathrm{M}$ ). The reaction mixture was warmed to room temperature over a period of 14 hours. Following this time, the stir bar was removed, and the reaction mixture was concentrated under reduced pressure. The resulting residue was purified by chromatography on silica gel (specific conditions are associated with each compound).

\section{Procedure $\mathrm{E}$}

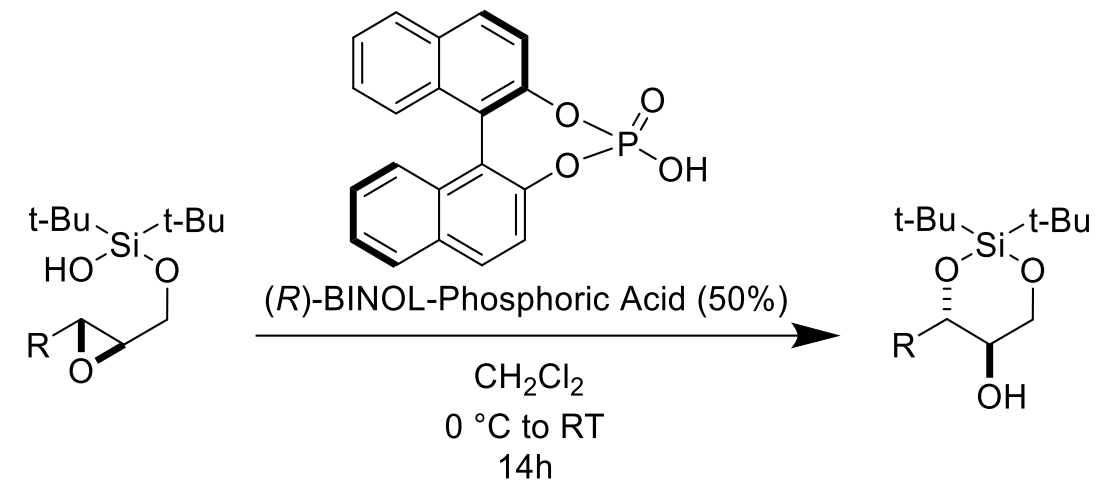

A $5 \mathrm{~mL}$ microwave vial was charged with a stir bar, silanol epoxide substrate $(0.2 \mathrm{mmol})$, and anhydrous $\mathrm{CH}_{2} \mathrm{Cl}_{2}(1 \mathrm{~mL})$. The homogenous mixture was cooled to $0{ }^{\circ} \mathrm{C}$ using an ice-water bath. $(R)$-BINOLPhosphoric acid (arbitrarily chosen) $(0.1 \mathrm{mmol}, 35 \mathrm{mg}, 0.5$ equivalents) was added in one portion, and the sides of the reaction flask were rinsed with an additional $1 \mathrm{~mL}$ of $\mathrm{CH}_{2} \mathrm{Cl}_{2}$ (Final concentration: $0.1 \mathrm{M}$ ). The reaction mixture was warmed to room temperature over a period of 14 hours. Following this time, the stir bar was removed, and the reaction mixture was concentrated under reduced pressure. The resulting residue was purified by chromatography on silica gel (specific conditions are associated with each compound).

\section{Procedure F}




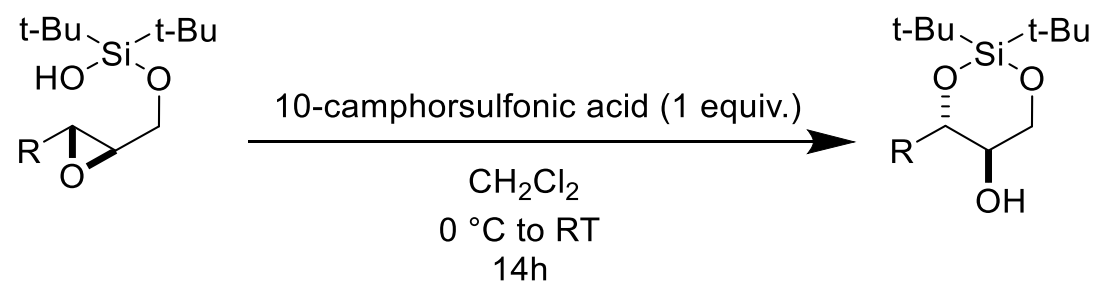

A $5 \mathrm{~mL}$ microwave vial was charged with a stir bar, silanol epoxide substrate $(0.132 \mathrm{mmol})$, and anhydrous $\mathrm{CH}_{2} \mathrm{Cl}_{2}(1 \mathrm{~mL})$. The homogenous mixture was cooled to $0{ }^{\circ} \mathrm{C}$ using an ice-water bath. $10-$ camphorsulfonic acid $(0.132 \mathrm{mmol}, 31 \mathrm{mg}, 1$ equivalent) was added in one portion, and the sides of the reaction flask were rinsed with an additional $0.5 \mathrm{~mL}$ of $\mathrm{CH}_{2} \mathrm{Cl}_{2}$ (Final concentration: $0.09 \mathrm{M}$ ). The reaction mixture was warmed to room temperature over a period of 14 hours. Following this time, the reaction mixture was transferred to a separatory funnel with $\mathrm{CH}_{2} \mathrm{Cl}_{2}$, and the organic layer was washed once with saturated aqueous $\mathrm{NaHCO}_{3}$ solution. The organic layer was collected, dried with $\mathrm{MgSO}_{4}$, and concentrated under reduced pressure. The resulting residue was purified by chromatography on silica gel (specific conditions are associated with each compound).

\section{Product Characterization Data}<smiles>CCC[C@H]1O[Si](C(C)(C)C)(C(C)(C)C)OC[C@H]1O</smiles>

2,2-di-tert-butyl-4-propyl-1,3,2-dioxasilinan-5-ol

Compound 25: Synthesized using Procedure A; Purified using a gradient of 0 to 25\% acetone/DCM on silica gel; single diastereomer; (colorless oil, $45 \mathrm{mg}, 80 \%$ yield); ${ }^{1} \mathrm{H} \mathrm{NMR}\left(400 \mathrm{MHz}, \mathrm{CDCl}_{3}\right) \delta 4.09$ (dd, $J=$ $10.5,4.6 \mathrm{~Hz}, 1 \mathrm{H}), 3.82-3.72(\mathrm{~m}, 2 \mathrm{H}), 3.53(\mathrm{ddd}, J=9.8,8.7,4.5 \mathrm{~Hz}, 1 \mathrm{H}), 1.82-1.71(\mathrm{~m}, 1 \mathrm{H}), 1.65-1.55$ $(\mathrm{m}, 1 \mathrm{H}), 1.51-1.38(\mathrm{~m}, 2 \mathrm{H}), 1.04(\mathrm{~s}, 9 \mathrm{H}), 0.98(\mathrm{~s}, 9 \mathrm{H}), 0.98-0.93(\mathrm{~m}, 3 \mathrm{H}) . ;{ }^{13} \mathrm{C}\left\{{ }^{1} \mathrm{H}\right\} \mathrm{NMR}\left(101 \mathrm{MHz}, \mathrm{CDCl}_{3}\right)$ $\delta$ 78.1, 70.8, 68.8, 36.7, 27.6, 27.2, 22.8, 20.1, 18.0, 14.1.; IR: 3426, 2953, 1145, $650.9 \mathrm{~cm}^{-1}$.; HRMS (ESI) $\mathrm{m} / \mathrm{z}:\left[\mathrm{M}+\mathrm{Na}^{+}\right]$calculated for $\mathrm{C}_{14} \mathrm{H}_{30} \mathrm{O}_{3} \mathrm{SiNa} 297.1862$, Found 297.1850 .<smiles>CC(C)C[C@H]1O[Si](C(C)(C)C)(C(C)(C)C)OC[C@H]1O</smiles>

2,2-di-tert-butyl-4-isobutyl-1,3,2-dioxasilinan-5-ol

Compound 26: Synthesized using Procedure B; Purified using a gradient of 0 to 20\% acetone/DCM on silica gel; single diastereomer; (colorless oil, $43 \mathrm{mg}, 74 \%$ yield); ${ }^{1} \mathrm{H} \mathrm{NMR}\left(400 \mathrm{MHz}, \mathrm{CDCl}_{3}\right) \delta 4.10$ (dd, $J=$ 10.5, $4.5 \mathrm{~Hz}, 1 \mathrm{H}$ ), $3.90-3.85(\mathrm{~m}, 1 \mathrm{H}), 3.85-3.76(\mathrm{~m}, 1 \mathrm{H}), 3.50$ (ddd, $J=10.0,8.7,4.6 \mathrm{~Hz}, 1 \mathrm{H}), 2.00$ (dpd, $J=9.3,6.7,4.5 \mathrm{~Hz}, 1 \mathrm{H}), 1.60(\mathrm{ddd}, J=13.4,9.3,2.3 \mathrm{~Hz}, 1 \mathrm{H}), 1.44-1.36(\mathrm{~m}, 1 \mathrm{H}), 1.07(\mathrm{~s}, 9 \mathrm{H}), 1.03-0.98$ 
(m, 12H), $0.97(\mathrm{~d}, J=6.6 \mathrm{~Hz}, 3 \mathrm{H}) .{ }^{13} \mathrm{C}\left\{{ }^{1} \mathrm{H}\right\} \mathrm{NMR}\left(101 \mathrm{MHz}, \mathrm{CDCl}_{3}\right) \delta 76.6,71.2,68.6,43.8,27.5,27.1,24.0$, 23.8, 22.6, 21.6, 19.9. IR 3374, 2934, 2866, 1473, 1364, $1073 \mathrm{~cm}^{-1}$; HRMS (APCI) m/z: [M + H+] calculated for C15H33O3Si 289.2193, Found 289.2194.<smiles>CC(C)CC[C@H]1O[Si](CC(C)(C)C)(C(C)(C)C)OCC1O</smiles>

2,2-di-tert-butyl-4-isopentyl-1,3,2-dioxasilinan-5-ol

Compound 27: Synthesized using Procedure B; Purified using a gradient of 0 to $20 \%$ acetone/DCM on silica gel; single diastereomer; (colorless oil, $50 \mathrm{mg}, 83 \%$ yield); $\left.{ }^{1} \mathrm{H} \mathrm{NMR} \mathrm{(400} \mathrm{MHz,} \mathrm{CDCl}_{3}\right) \delta 4.10$ (dd, $J=$ 10.5, $4.5 \mathrm{~Hz}, 1 \mathrm{H}$ ), $3.85-3.72(\mathrm{~m}, 2 \mathrm{H}$ ), 3.55 (ddd, $J=10.0,8.7,4.5 \mathrm{~Hz}, 1 \mathrm{H}$ ), 1.85 (dddd, $J=13.0,10.3,4.6$, $2.0 \mathrm{~Hz}, 1 \mathrm{H}), 1.61(\mathrm{dp}, J=13.1,6.6 \mathrm{~Hz}, 1 \mathrm{H}), 1.55-1.37(\mathrm{~m}, 2 \mathrm{H}), 1.37-1.27(\mathrm{~m}, 1 \mathrm{H}), 1.06(\mathrm{~s}, 9 \mathrm{H}), 1.01(\mathrm{~s}$, 9H), 0.94 (dd, $J=6.6,4.0 \mathrm{~Hz}, 6 \mathrm{H}) .{ }^{13} \mathrm{C}\left\{{ }^{1} \mathrm{H}\right\} \mathrm{NMR}\left(101 \mathrm{MHz}, \mathrm{CDCl}_{3}\right) \delta 78.6,70.7,68.6,33.9,32.3,27.9,27.5$, 27.1, 22.8, 22.6, 22.5, 20.0. IR 3340, 2974, 2871, 1473, 1061, $822 \mathrm{~cm}^{-1}$.; HRMS (ESI) m/z: [M + Na+] calculated for $\mathrm{C} 16 \mathrm{H} 34 \mathrm{NaO} 3 \mathrm{Si} 325.2169$ Found 325.2188.<smiles>CC(C)[C@H]1O[Si](C(C)(C)C)(C(C)(C)C)OCC1O</smiles>

2,2-di-tert-butyl-4-isopropyl-1,3,2-dioxasilinan-5-ol

Compound 28: Synthesized using Procedure B; Purified using a gradient of 0 to $30 \%$ Acetone/DCM on silica gel; single diastereomer; (colorless oil, crystallizes upon standing, $39 \mathrm{mg}, 71 \%$ yield); ${ }^{1} \mathrm{H}$ NMR (400 $\left.\mathrm{MHz}, \mathrm{CDCl}_{3}\right) \delta 4.17-4.09(\mathrm{~m}, 1 \mathrm{H}), 3.82-3.66(\mathrm{~m}, 3 \mathrm{H}), 2.10(\mathrm{pd}, J=6.8,1.9 \mathrm{~Hz}, 1 \mathrm{H}), 1.10-1.04(\mathrm{~m}, 12 \mathrm{H})$, $1.02(\mathrm{~s}, 9 \mathrm{H}), 0.95(\mathrm{~d}, J=6.8 \mathrm{~Hz}, 3 \mathrm{H}) .{ }^{13} \mathrm{C}\left\{{ }^{1} \mathrm{H}\right\} \mathrm{NMR}\left(101 \mathrm{MHz}, \mathrm{CDCl}_{3}\right) \delta 81.8,69.2,67.8,29.0,27.6,27.1$, $22.8,20.3,19.5,14.5$. IR 3340, 2963, 2860, 1473, $1050 \mathrm{~cm}^{-1}$; HRMS (APCI) m/z: [M + H $\mathrm{H}^{+}$calculated for C14H31O3Si 275.2037, Found 275.2028.<smiles>CCC(CC)[C@H]1O[Si](C)(C(C)(C)C)OC[C@H]1O</smiles>

2,2-di-tert-butyl-4-(pentan-3-yl)-1,3,2-dioxasilinan-5-ol

Compound 29: Synthesized using Procedure B; purified using a gradient of 0 to $30 \%$ Acetone/DCM on silica gel; single diastereomer; (colorless oil, $31 \mathrm{mg}, 52 \%$ yield); $\left.{ }^{1} \mathrm{H} \mathrm{NMR} \mathrm{(400} \mathrm{MHz,} \mathrm{CDCl}_{3}\right) \delta 4.23-4.10$ $(\mathrm{m}, 1 \mathrm{H}), 3.99-3.92(\mathrm{~m}, 1 \mathrm{H}), 3.90-3.73(\mathrm{~m}, 2 \mathrm{H}), 1.66-1.45(\mathrm{~m}, 4 \mathrm{H}), 1.36-1.25(\mathrm{~m}, 1 \mathrm{H}), 1.09-1.02(\mathrm{~m}$, $12 \mathrm{H}), 1.02-0.90(\mathrm{~m}, 12 \mathrm{H}) .{ }^{13} \mathrm{C}\left\{{ }^{1} \mathrm{H}\right\} \mathrm{NMR}\left(101 \mathrm{MHz}, \mathrm{CDCl}_{3}\right) \delta 78.9,69.4,67.2,42.5,27.6,27.1,22.8,22.4$, 20.3, 20.2, 12.5, 11.8. IR 3402, 2963, 2940, 2860, 1467, $1061 \mathrm{~cm}^{-1}$; HRMS (ESI) m/z: [M + Na+] calculated for C16H34NaO3Si 325.2169 Found 325.2181. 


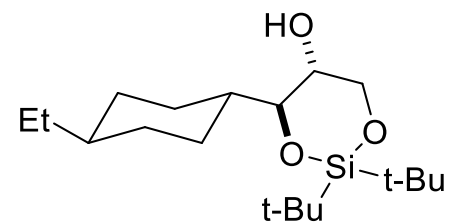

2,2-di-tert-butyl-4-((1r,4S)-4-ethylcyclohexyl)-1,3,2-dioxasilinan-5-ol

Compound 30: Synthesized using Procedure B; Purified using a gradient of 0 to $25 \%$ acetone/DCM on silica gel; single diastereomer; (white semi-solid, $50 \mathrm{mg}, 71 \%$ yield); ${ }^{1} \mathrm{H} \mathrm{NMR}\left(600 \mathrm{MHz}, \mathrm{CDCl}_{3}\right) \delta 4.18-$ $4.10(\mathrm{~m}, 1 \mathrm{H}), 3.81-3.75(\mathrm{~m}, 2 \mathrm{H}), 3.72(\mathrm{dt}, J=7.3,2.0 \mathrm{~Hz}, 1 \mathrm{H}), 1.90-1.79(\mathrm{~m}, 2 \mathrm{H}), 1.76-1.67(\mathrm{~m}, 2 \mathrm{H})$, $1.58(\mathrm{td}, J=9.7,3.4 \mathrm{~Hz}, 2 \mathrm{H}), 1.36(\mathrm{qd}, J=13.0,3.4 \mathrm{~Hz}, 1 \mathrm{H}), 1.24(\mathrm{p}, J=7.2 \mathrm{~Hz}, 2 \mathrm{H}), 1.17-0.92(\mathrm{~m}, 3 \mathrm{H})$, $1.06(\mathrm{~s}, 9 \mathrm{H}), 1.01(\mathrm{~s}, 9 \mathrm{H}), 0.91(\mathrm{t}, J=7.4 \mathrm{~Hz}, 3 \mathrm{H}) .{ }^{13} \mathrm{C}\left\{{ }^{1} \mathrm{H}\right\} \mathrm{NMR}\left(101 \mathrm{MHz}, \mathrm{CDCl}_{3}\right) \delta 81.6,69.3,67.1,39.4$, 39.2, 32.9, 32.5, 30.0, 29.6, 27.6, 27.1, 24.8, 22.8, 20.3, 11.5. IR 3351, 2934, 2854, 1473, $1056 \mathrm{~cm}^{-1}$; HRMS (APCl) m/z: [M + $\mathrm{H}^{+}$] calculated for $\mathrm{C} 19 \mathrm{H} 3903 \mathrm{Si}+343.2663$, Found 343.2658.

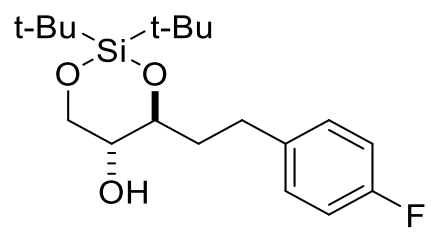

2,2-di-tert-butyl-4-(4-fluorophenethyl)-1,3,2-dioxasilinan-5-ol

Compound 31: Synthesized using Procedure A; Purified using a gradient of 0 to 25\% acetone/DCM on silica gel; single diastereomer; (colorless oil, $51.4 \mathrm{mg}, 72 \%$ yield); ${ }^{1} \mathrm{H} \mathrm{NMR}\left(600 \mathrm{MHz}, \mathrm{CDCl}_{3}\right) \delta 7.24-7.19$ (m, 2H), $7.03-6.96(\mathrm{~m}, 2 \mathrm{H}), 4.10(\mathrm{dd}, J=10.5,4.6 \mathrm{~Hz}, 1 \mathrm{H}), 3.82-3.70(\mathrm{~m}, 2 \mathrm{H}), 3.58$ (ddd, $J=10.0,8.8$, 4.6 Hz, 1H), 2.91 (ddd, $J=13.9,9.2,4.8 \mathrm{~Hz}, 1 \mathrm{H}), 2.76(\mathrm{dt}, J=13.8,8.3 \mathrm{~Hz}, 1 \mathrm{H}$ ), 2.17 (dddd, $J=13.5,9.2$, 7.7, $2.4 \mathrm{~Hz}, 1 \mathrm{H}), 1.75(\mathrm{dtd}, J=13.8,9.1,4.8 \mathrm{~Hz}, 1 \mathrm{H}), 1.07(\mathrm{~s}, 9 \mathrm{H}), 1.04(\mathrm{~s}, 9 \mathrm{H}) .{ }^{13} \mathrm{C}\left\{{ }^{1} \mathrm{H}\right\} \mathrm{NMR}(126 \mathrm{MHz}$, $\left.\mathrm{CDCl}_{3}\right) \delta 161.2(\mathrm{~d}, J=243.1 \mathrm{~Hz}), 137.8(\mathrm{~d}, J=3.3 \mathrm{~Hz}), 129.9(\mathrm{~d}, J=7.7 \mathrm{~Hz}), 115.0(\mathrm{~d}, J=21.0 \mathrm{~Hz}), 77.2$, 70.7, 68.6, 36.5, 30.1, 27.4, 27.1, 22.6, 20.0. IR 3420, 2934, 2860, 1507, 1061, $827 \mathrm{~cm}^{-1}$.; HRMS (APCl) $\mathrm{m} / \mathrm{z}:\left[\mathrm{M}+\mathrm{H}^{+}\right]$calculated for C19H32FO3Si 355.2099, Found 355.2090.<smiles>CC(C)(C)[Si]1(C(C)(C)C)OC[C@@H](O)C(CCc2ccc(Br)cc2)O1</smiles>

4-(4-bromophenethyl)-2,2-di-tert-butyl-1,3,2-dioxasilinan-5-ol

Compound 32: Synthesized using Procedure B; Purified using a gradient of 0 to $20 \%$ acetone/DCM on silica gel; single diastereomer; (colorless oil, $56 \mathrm{mg}, 70 \%$ yield); $\left.{ }^{1} \mathrm{H} \mathrm{NMR} \mathrm{(400} \mathrm{MHz,} \mathrm{CDCl}_{3}\right) \delta 7.43-7.36$ $(\mathrm{m}, 2 \mathrm{H}), 7.14-7.07(\mathrm{~m}, 2 \mathrm{H}), 4.06(\mathrm{dd}, J=10.5,4.6 \mathrm{~Hz}, 1 \mathrm{H}), 3.79-3.67(\mathrm{~m}, 2 \mathrm{H}), 3.54(\mathrm{td}, J=9.4,4.6 \mathrm{~Hz}$, $1 \mathrm{H}), 2.86(\mathrm{ddd}, J=13.9,9.2,4.9 \mathrm{~Hz}, 1 \mathrm{H}), 2.71(\mathrm{dt}, J=13.8,8.3 \mathrm{~Hz}, 1 \mathrm{H}), 2.13$ (dddd, $J=13.5,9.2,7.7,2.5$ $\mathrm{Hz}, 1 \mathrm{H}), 1.78-1.65(\mathrm{~m}, 1 \mathrm{H}), 1.04(\mathrm{~s}, 9 \mathrm{H}), 1.00(\mathrm{~s}, 9 \mathrm{H}) . ;{ }^{13} \mathrm{C}\left\{{ }^{1} \mathrm{H}\right\} \mathrm{NMR}\left(101 \mathrm{MHz}, \mathrm{CDCl}_{3}\right) \delta$ 141.4, 131.5, 130.6, 119.6, 77.3, 70.8, 68.7, 36.4, 30.5, 27.6, 27.3, 22.8, 20.2.; IR: 3436, 2963, 2860, 1475, $650.9 \mathrm{~cm}^{-1}$.; HRMS (ESI) m/z: [M + Na+] calculated for $\mathrm{C}_{19} \mathrm{H}_{31} \mathrm{BrO}_{3} \mathrm{SiNa}$ 437.1124, Found Mass 437.1127. 
<smiles>CC(C)(C)[Si]1(C(C)(C)C)OC[C@@H](O)C(CCc2cccc(C(F)(F)F)c2)O1</smiles>

2,2-di-tert-butyl-4-(3-(trifluoromethyl)phenethyl)-1,3,2-dioxasilinan-5-ol

Compound 33: Synthesized using the Procedure B; Purified using a gradient of 0 to $25 \%$ Acetone/DCM

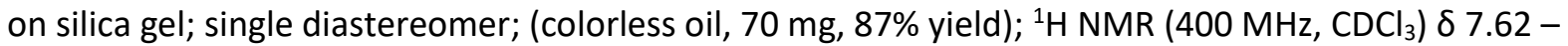
$7.37(\mathrm{~m}, 4 \mathrm{H}), 4.13-4.04(\mathrm{~m}, 1 \mathrm{H}), 3.85-3.69(\mathrm{~m}, 2 \mathrm{H}), 3.59(\mathrm{ddd}, J=10.0,8.7,4.6 \mathrm{~Hz}, 1 \mathrm{H}), 2.99$ (ddd, $J=$ 13.9, 9.0, $5.0 \mathrm{~Hz}, 1 \mathrm{H}), 2.87(\mathrm{dt}, J=13.8,8.2 \mathrm{~Hz}, 1 \mathrm{H}), 2.27-2.18(\mathrm{~m}, 1 \mathrm{H}), 1.79$ (dddd, $J=13.6,9.6,8.7,5.0$ $\mathrm{Hz}, 1 \mathrm{H}), 1.07$ (s, 9H), $1.03(\mathrm{~s}, 9 \mathrm{H}) .{ }^{13} \mathrm{C}\left\{{ }^{1} \mathrm{H}\right\} \mathrm{NMR}\left(126 \mathrm{MHz}, \mathrm{CDCl}_{3}\right) \delta 142.1,130.9,129.7$ (q, $\left.J=30 \mathrm{~Hz}\right)$, 127.7, $124.4(\mathrm{~m}), 122.1$ (q, $J=210 \mathrm{~Hz}), 121.6(\mathrm{~m}), 76.0,69.6,67.5,35.1,29.8,26.4,26.0,21.6,18.9 . \mathrm{IR}$ $302,2934,2860,1330,1073,827 \mathrm{~cm}^{-1}$.; HRMS (ESI) m/z: [M + Na+] calculated for C2OH31F3NaO3Si 427.1887 Found 427.1901.<smiles>COc1ccc(CCCC2O[Si](C(C)(C)C)(C(C)(C)C)OC[C@H]2O)cc1</smiles>

2,2-di-tert-butyl-4-(3-(4-methoxyphenyl)propyl)-1,3,2-dioxasilinan-5-ol

Compound 34: synthesized using Procedure A; Purified using a gradient of 0 to 30\% Acetone/DCM on silica gel; single diastereomer; (colorless oil, $57 \mathrm{mg}, 70 \%$ yield); ${ }^{1} \mathrm{H} \mathrm{NMR}\left(400 \mathrm{MHz}, \mathrm{CDCl}_{3}\right) \delta 7.20-7.10$ $(\mathrm{m}, 2 \mathrm{H}), 6.92-6.76(\mathrm{~m}, 2 \mathrm{H}), 4.10(\mathrm{dd}, J=10.5,4.6 \mathrm{~Hz}, 1 \mathrm{H}), 3.87-3.73(\mathrm{~m}, 5 \mathrm{H}), 3.54$ (ddd, $J=10.1,8.8$, $4.6 \mathrm{~Hz}, 1 \mathrm{H}), 2.71-2.58(\mathrm{~m}, 2 \mathrm{H}), 1.95-1.84(\mathrm{~m}, 2 \mathrm{H}), 1.79-1.67(\mathrm{~m}, 1 \mathrm{H}), 1.54-1.44(\mathrm{~m}, 1 \mathrm{H}), 1.10-1.04$ (m, 9H), $1.01(\mathrm{~s}, 9 \mathrm{H}) .{ }^{13} \mathrm{C}\left\{{ }^{1} \mathrm{H}\right\} \mathrm{NMR}\left(101 \mathrm{MHz}, \mathrm{CDCl}_{3}\right) \delta$ 157.6, 134.7, 129.2, 113.7, 78.1, 70.6, 68.6, 55.2, $34.8,34.0,27.5,27.1,26.8,22.6,20.0$. IR 3442, 2934, 2866, 1513, 1467, 1067, $872 \mathrm{~cm}^{-1}$.; HRMS (ESI) $\mathrm{m} / \mathrm{z}$ : [M-H] calculated for C21H35O4Si 379.2310 Found 379.2351.

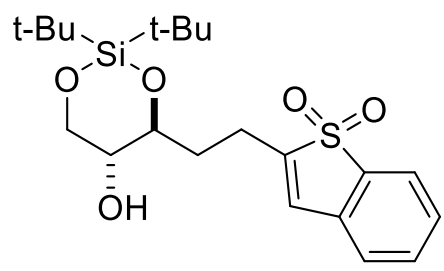

2-(2-(2,2-di-tert-butyl-5-hydroxy-1,3,2-dioxasilinan-4-yl)ethyl)benzo[b]thiophene 1,1-dioxide

Compound 35: Synthesized using Procedure B; Purified using a gradient of 0 to $25 \%$ acetone/DCM on silica gel; single diastereomer; (white solid, $69 \mathrm{mg}, 81 \%$ yield); ${ }^{1} \mathrm{H} \mathrm{NMR}\left(400 \mathrm{MHz}, \mathrm{CDCl}_{3}\right) \delta 7.72$ (dq, $J=$ 7.4, $0.9 \mathrm{~Hz}, 1 \mathrm{H}), 7.54(\mathrm{td}, J=7.5,1.2 \mathrm{~Hz}, 1 \mathrm{H}), 7.46(\mathrm{td}, J=7.5,1.1 \mathrm{~Hz}, 1 \mathrm{H}), 7.30(\mathrm{dt}, J=7.5,0.9 \mathrm{~Hz}, 1 \mathrm{H})$, $6.86(\mathrm{td}, J=1.7,0.8 \mathrm{~Hz}, 1 \mathrm{H}), 4.16-4.05(\mathrm{~m}, 1 \mathrm{H}), 3.89(\mathrm{td}, J=9.0,2.6 \mathrm{~Hz}, 1 \mathrm{H}), 3.79(\mathrm{t}, J=10.3 \mathrm{~Hz}, 1 \mathrm{H})$, 3.59 (ddd, $J=10.1,9.0,4.7 \mathrm{~Hz}, 1 \mathrm{H}$ ), 2.92 (dddd, $J=16.3,9.1,5.0,1.9 \mathrm{~Hz}, 1 \mathrm{H}$ ), 2.76 (dddd, $J=16.4,8.8$, 7.0, 1.7 Hz, 1H), 2.38 (dddd, $J=13.6,9.4,7.1,2.6 \mathrm{~Hz}, 1 \mathrm{H}), 1.91-1.73(\mathrm{~m}, 1 \mathrm{H}), 1.06(\mathrm{~s}, 9 \mathrm{H}), 1.02(\mathrm{~s}, 9 \mathrm{H})$. ${ }^{13} \mathrm{C}\left\{{ }^{1} \mathrm{H}\right\}$ NMR $\left(101 \mathrm{MHz}, \mathrm{CDCl}_{3}\right) \delta$ 145.0, 136.7, 133.6, 131.5, 129.4, 125.7, 124.3, 121.4, 77.3, 70.2, 68.6, 31.6, 27.5, 27.1, 22.6, 19.9. IR 2963, 2860, 1541, 1284, 1147, 1067, $822 \mathrm{~cm}^{-1}$.; HRMS (APCI) m/z: [M + H ] calculated for $\mathrm{C} 21 \mathrm{H} 33 \mathrm{O} 5 \mathrm{SS}+425.1818$, Found 425.1796. 


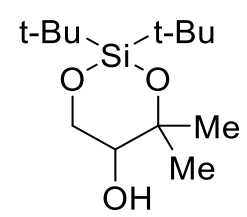

2,2-di-tert-butyl-4,4-dimethyl-1,3,2-dioxasilinan-5-ol

Compound 36: Synthesized using Procedure B; Purified using a gradient of 0 to 25\% acetone/DCM on silica gel; (colorless oil, $38 \mathrm{mg}, 74 \%$ yield); ${ }^{1} \mathrm{H}$ NMR $\left(400 \mathrm{MHz}, \mathrm{CDCl}_{3}\right) \delta 4.02$ (dd, $J=11.1,3.7 \mathrm{~Hz}, 1 \mathrm{H}$ ), 3.93 (dd, $J=11.0,8.7 \mathrm{~Hz}, 1 \mathrm{H}$ ), 3.70 (dd, $J=8.6,3.8 \mathrm{~Hz}, 1 \mathrm{H}), 1.40(\mathrm{~s}, 3 \mathrm{H}), 1.32(\mathrm{~s}, 3 \mathrm{H}), 1.05(\mathrm{~s}, 9 \mathrm{H}), 1.04(\mathrm{~s}, 9 \mathrm{H})$. ${ }^{13} \mathrm{C}\left\{{ }^{1} \mathrm{H}\right\}$ NMR $\left(101 \mathrm{MHz}, \mathrm{CDCl}_{3}\right) \delta 75.8,74.1,64.8,29.5,27.6,27.5,23.5,21.2,20.7$. IR 3448, 2974, 2866, 1473, 1153, $1044 \mathrm{~cm}^{-1}$; HRMS (APCl) m/z: [M + H+] calculated for $\mathrm{C} 13 \mathrm{H} 2903 \mathrm{Si} 261.1886$, Found 261.1896.<smiles>CC[C@H](O)C1CCO[Si](C(C)(C)C)(C(C)(C)C)O1</smiles>

(2,2-di-tert-butyl-1,3,2-dioxasilinan-4-yl) propan-1-ol

Compound 37: Synthesized using Procedure B; Purified using a gradient of 0 to $25 \%$ acetone/DCM on silica gel; single diastereomer; (colorless oil, $33 \mathrm{mg}, 65 \%$ yield); ${ }^{1} \mathrm{H} \mathrm{NMR}\left(400 \mathrm{MHz}, \mathrm{CDCl}_{3}\right) \delta 4.21-4.06(\mathrm{~m}, 2 \mathrm{H})$, 4.02 (ddd, $J=11.3,4.4,2.1 \mathrm{~Hz}, 1 \mathrm{H}$ ), 3.49 (dt, $J=8.5,4.2 \mathrm{~Hz}, 1 \mathrm{H}$ ), 1.92 (dtd, $J=14.1,11.7,5.1 \mathrm{~Hz}, 1 \mathrm{H}), 1.61$ (dq, $J=14.2,2.3 \mathrm{~Hz}, 1 \mathrm{H}), 1.56-1.40(\mathrm{~m}, 2 \mathrm{H}), 1.07-0.97(\mathrm{~m}, 21 \mathrm{H}) . ;{ }^{13} \mathrm{C}\left\{{ }^{1} \mathrm{H}\right\} \mathrm{NMR}\left(101 \mathrm{MHz}, \mathrm{CDCl}_{3}\right) \delta 77.0$, 76.3, 64.6, 30.7, 27.6, 27.3, 24.8, 22.9, 20.1, 10.4.; IR: 3436, 2963, 2880, 1146, $650.9 \mathrm{~cm}^{-1}$.; HRMS (ESI) $\mathrm{m} / \mathrm{z}:\left[\mathrm{M}+\mathrm{Na}^{+}\right]$calculated for $\mathrm{C}_{14} \mathrm{H}_{30} \mathrm{O}_{3} \mathrm{SiNa} 297.1862$, Found 297.1844.

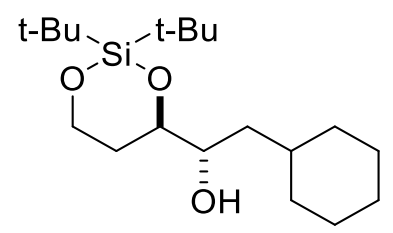

2-cyclohexyl-1-(2,2-di-tert-butyl-1,3,2-dioxasilinan-4-yl)ethan-1-ol

Compound 38: Synthesized with Procedure B but kept at $0{ }^{\circ} \mathrm{C}$ for $45 \mathrm{~min}$.; Purified using a gradient of 0 to $20 \%$ acetone/DCM on silica gel; single diastereomer; (colorless oil, $33 \mathrm{mg}, 50 \%$ yield); ${ }^{1} \mathrm{H}$ NMR (400 $\mathrm{MHz}, \mathrm{CDCl}_{3}$ ) $\delta 4.23-4.05(\mathrm{~m}, 2 \mathrm{H}$ ), 4.00 (ddd, $J=11.3,4.1,2.1 \mathrm{~Hz}, 1 \mathrm{H}$ ), 3.74 (ddd, $J=9.6,4.1,3.2 \mathrm{~Hz}, 1 \mathrm{H}$ ), $2.03-1.96(\mathrm{~m}, 1 \mathrm{H}), 1.96-1.90(\mathrm{~m}, 1 \mathrm{H}), 1.86(\mathrm{ddt}, J=11.4,4.0,2.1 \mathrm{~Hz}, 1 \mathrm{H}), 1.79-1.65(\mathrm{~m}, 4 \mathrm{H}), 1.61(\mathrm{dq}$, $J=14.2,2.2 \mathrm{~Hz}, 1 \mathrm{H}$ ), 1.54 (dddd, $J=15.9,11.7,7.2,3.4 \mathrm{~Hz}, 1 \mathrm{H}), 1.41-1.10(\mathrm{~m}, 4 \mathrm{H}), 1.06(\mathrm{~s}, 9 \mathrm{H}), 1.03(\mathrm{~s}$, $9 \mathrm{H}), 1.01-0.94(\mathrm{~m}, 1 \mathrm{H}), 0.94-0.79(\mathrm{~m}, 1 \mathrm{H}) .{ }^{13} \mathrm{C}\left\{{ }^{1} \mathrm{H}\right\}$ NMR $\left(101 \mathrm{MHz}, \mathrm{CDCl}_{3}\right) \delta 77.5,72.1,64.4,39.4,34.5$, $34.1,32.7,30.2,27.4,27.2,26.6,26.4,26.2,22.8,20.0$. IR 3362, 2929, 2854, 1473, 1113, $827 \mathrm{~cm}^{-1}$.; HRMS (ESI) m/z: [M + Na+] calculated for C19H38NaO3Si 365.2482 Found 365.2502. 
<smiles>CO[C@H](O)[C@H]1CCO[Si](C(C)(C)C)(C(C)(C)C)O1</smiles>

(2,2-di-tert-butyl-1,3,2-dioxasilinan-4-yl) propan-1-ol

Compound 39: Synthesized using Procedure B; Purified using a gradient of 0 to 25\% acetone/DCM on silica gel; single diastereomer; (colorless oil, $35 \mathrm{mg}, 60 \%$ yield); ${ }^{1} \mathrm{H} \mathrm{NMR}\left(400 \mathrm{MHz}, \mathrm{CDCl}_{3}\right) \delta 4.17-4.06$ $(\mathrm{m}, 2 \mathrm{H}), 3.95-3.83(\mathrm{~m}, 1 \mathrm{H}), 3.30(\mathrm{dt}, J=9.5,5.2 \mathrm{~Hz}, 1 \mathrm{H}), 2.65(\mathrm{~s}, 1 \mathrm{H}), 1.92(\mathrm{dtd}, J=14.2,10.9,6.3 \mathrm{~Hz}$, 1H), $1.64-1.47(\mathrm{~m}, 2 \mathrm{H}), 1.42(\mathrm{ddt}, J=13.7,8.2,7.3 \mathrm{~Hz}, 1 \mathrm{H}), 1.02(\mathrm{dd}, J=13.6,2.4 \mathrm{~Hz}, 21 \mathrm{H}) . ;{ }^{13} \mathrm{C}\left\{{ }^{1} \mathrm{H}\right\} \mathrm{NMR}$ $\left(101 \mathrm{MHz}, \mathrm{CDCl}_{3}\right) \delta$ 77.1, 76.6, 64.2, 32.7, 27.5, 27.3, 25.7, 22.9, 20.0, 10.1.; IR: 3446, 2973, 2890, 1150, $651 \mathrm{~cm}^{-1}$.; HRMS (ESI) m/z: [M + Na+] calculated for $\mathrm{C}_{14} \mathrm{H}_{30} \mathrm{O}_{3} \mathrm{SiNa} 297.1862$, Found 297.1872.<smiles>CCCC1O[Si](C(C)(C)C)(C(C)(C)C)OC[C@H]1O</smiles>

2,2-di-tert-butyl-4-propyl-1,3,2-dioxasilinan-5-ol

Compound 40: Synthesized using Procedure C; Purified using a gradient of 0 to $25 \%$ acetone/DCM on silica gel; single diastereomer; (colorless oil, $23 \mathrm{mg}, 40 \%$ yield); ${ }^{1} \mathrm{H} \mathrm{NMR}\left(500 \mathrm{MHz}, \mathrm{CDCl}_{3}\right) \delta 4.27-4.21(\mathrm{~m}, 1 \mathrm{H})$, $4.13(\mathrm{ddd}, J=12.0,6.1,3.7 \mathrm{~Hz}, 2 \mathrm{H}), 3.48(\mathrm{~s}, 1 \mathrm{H}), 2.41-2.17(\mathrm{~m}, 1 \mathrm{H}), 1.74-1.64(\mathrm{~m}, 1 \mathrm{H}), 1.55-1.33(\mathrm{~m}$, 3H), $1.06(\mathrm{~m}, 18 \mathrm{H}), 0.95(\mathrm{td}, J=7.1,1.7 \mathrm{~Hz}, 3 \mathrm{H}) . ;{ }^{13} \mathrm{C} \mathrm{NMR}\left(126 \mathrm{MHz}, \mathrm{CDCl}_{3}\right) \delta 76.1,71.0,70.3,36.3,28.1$, 27.1, 23.3, 20.8, 18.7, 14.1.; IR: 3426, 2957, 1473, 1159, $650.9 \mathrm{~cm}^{-1}$.; HRMS (ESI) m/z: [M + Na+] calculated for $\mathrm{C}_{14} \mathrm{H}_{30} \mathrm{O}_{3} \mathrm{SiNa}^{+}$297.1862, Found 297.1861.<smiles>CC(C)(C)[Si]1(C(C)(C)C)OC[C@@H](O)C(c2ccccc2)O1</smiles>

\section{2,2-di-tert-butyl-4-phenyl-1,3,2-dioxasilinan-5-ol}

Compound 41: Synthesized using Procedure D; Single diastereomer; Purified using a gradient of 0 to $30 \% \mathrm{EtOAc/hexanes}$ on silica gel; (crystalline solid, $48 \mathrm{mg}, 80 \%$ yield); $\left.{ }^{1} \mathrm{H} \mathrm{NMR} \mathrm{(400} \mathrm{MHz}, \mathrm{CDCl}_{3}\right) \delta 7.56-$ $7.33(\mathrm{~m}, 5 \mathrm{H}), 4.81(\mathrm{~d}, J=9.1 \mathrm{~Hz}, 1 \mathrm{H}), 4.25(\mathrm{dd}, J=10.6,4.8 \mathrm{~Hz}, 1 \mathrm{H}), 4.00(\mathrm{t}, J=10.4 \mathrm{~Hz}, 1 \mathrm{H}), 3.69$ (dddd, $J$ $=10.4,9.1,4.8,1.4 \mathrm{~Hz}, 1 \mathrm{H}), 1.14(\mathrm{~s}, 9 \mathrm{H}), 1.13(\mathrm{~s}, 9 \mathrm{H}) .{ }^{13} \mathrm{C}\left\{{ }^{1} \mathrm{H}\right\} \mathrm{NMR}\left(101 \mathrm{MHz}, \mathrm{CDCl}_{3}\right) \delta 141.1,128.6$, $128.3,126.9,80.7,71.6,68.6,27.6,27.3,22.8,20.3$. IR 3420, 2964, 2860, 1472, 1124, $827 \mathrm{~cm}^{-1}$.; HRMS (ESI) $\mathrm{m} / \mathrm{z}:\left[\mathrm{M}+\mathrm{Na}^{+}\right]$calculated for $\mathrm{C} 17 \mathrm{H} 28 \mathrm{NaO} 3 \mathrm{Si} 331.1700$, Found 331.1729. 


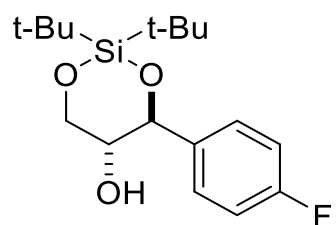

2,2-di-tert-butyl-4-(4-fluorophenyl)-1,3,2-dioxasilinan-5-ol

Compound 42: Synthesized using Procedure E; Purified using a gradient of 0 to 20\% EtOAc/hexanes on silica gel; single diastereomer; (white solid, $41 \mathrm{mg}, 63 \%$ yield); ${ }^{1} \mathrm{H} \mathrm{NMR}\left(400 \mathrm{MHz}, \mathrm{CDCl}_{3}\right) \delta 7.53-7.38$ $(\mathrm{m}, 2 \mathrm{H}), 7.17-7.02(\mathrm{~m}, 2 \mathrm{H}), 4.79(\mathrm{~d}, J=9.1 \mathrm{~Hz}, 1 \mathrm{H}), 4.23(\mathrm{dd}, J=10.6,4.9 \mathrm{~Hz}, 1 \mathrm{H}), 3.98(\mathrm{t}, J=10.4 \mathrm{~Hz}$, 1H), 3.65 (ddd, $J=10.2,9.1,4.9 \mathrm{~Hz}, 1 \mathrm{H}), 1.13(\mathrm{~s}, 9 \mathrm{H}), 1.12(\mathrm{~s}, 9 \mathrm{H}) .{ }^{13} \mathrm{C}\left\{{ }^{1} \mathrm{H}\right\} \mathrm{NMR}\left(101 \mathrm{MHz}, \mathrm{CDCl}_{3}\right) \delta 162.6$ (d, $J=246.5 \mathrm{~Hz}$ ), 137.0 (d, J = 3.1 Hz), 128.5 (d, J = 8.1 Hz), 115.4 (d, $J=21.4 \mathrm{~Hz}$ ), 80.0, 71.6, 68.6, 27.6, 27.2, 22.8, 20.3. IR 2946, 2866, 1518, $1061 \mathrm{~cm}^{-1}$.; HRMS (ESI) m/z: [M-H] calculated for C17H26FO3Si 325.1641, Found 325.1633.<smiles>CC(C)(C)[Si]1(C(C)(C)C)OC[C@@H](O)C(c2ccc(Br)cc2)O1</smiles>

4-(4-bromophenyl)-2,2-di-tert-butyl-1,3,2-dioxasilinan-5-ol

Compound 43: Synthesized using Procedure D on a $0.189 \mathrm{mmol}$ scale; Purified using a gradient of 0 to $20 \% \mathrm{EtOAc/hexanes} \mathrm{on} \mathrm{silica} \mathrm{gel;} \mathrm{single} \mathrm{diastereomer;} \mathrm{(colorless} \mathrm{oil,} 62 \mathrm{mg}, 85 \%$ yield); ${ }^{1} \mathrm{H}$ NMR (400 $\left.\mathrm{MHz}, \mathrm{CDCl}_{3}\right) \delta 7.60-7.52(\mathrm{~m}, 2 \mathrm{H}), 7.43-7.32(\mathrm{~m}, 2 \mathrm{H}), 4.78(\mathrm{~d}, J=9.1 \mathrm{~Hz}, 1 \mathrm{H}), 4.23(\mathrm{dd}, J=10.6,4.8 \mathrm{~Hz}$, $1 \mathrm{H}), 3.98(\mathrm{t}, J=10.4 \mathrm{~Hz}, 1 \mathrm{H}), 3.65(\mathrm{ddd}, J=10.2,9.1,4.8 \mathrm{~Hz}, 1 \mathrm{H}), 1.12(\mathrm{~m}, 18 \mathrm{H}) .{ }^{13} \mathrm{C}\left\{{ }^{1} \mathrm{H}\right\} \mathrm{NMR}(101 \mathrm{MHz}$, $\left.\mathrm{CDCl}_{3}\right) \delta 140.3,131.6,128.6,122.1,80.0,71.5,68.6,27.5,27.2,22.8,20.3$. IR 3420, 2934, 2860, 1473, $1124,1073,827 \mathrm{~cm}^{-1}$; HRMS (ESI) m/z: [M + Na ${ }^{+}$calculated for $\mathrm{C} 17 \mathrm{H} 27 \mathrm{BrNaO} S \mathrm{Si} 409.0805$ Found 409.0839 .<smiles>CC(C)(C)[Si]1(C(C)(C)C)OC[C@@H](O)C(c2ccc(Cl)cc2)O1</smiles>

2,2-di-tert-butyl-4-(4-chlorophenyl)-1,3,2-dioxasilinan-5-ol

Compound 44: Synthesized using Procedure D; Purified using a gradient of 0 to $20 \%$ EtOAc/hexanes on silica gel; single diastereomer; (colorless oil, crystallizes upon standing; $57 \mathrm{mg} ; 82 \%$ yield); ${ }^{1} \mathrm{H}$ NMR (400 $\left.\mathrm{MHz}, \mathrm{CDCl}_{3}\right) \delta 7.43(\mathrm{~d}, J=8.6 \mathrm{~Hz}, 2 \mathrm{H}), 7.41-7.37(\mathrm{~m}, 2 \mathrm{H}), 4.79(\mathrm{~d}, J=9.1 \mathrm{~Hz}, 1 \mathrm{H}), 4.22(\mathrm{dd}, J=10.6,4.8$ $\mathrm{Hz}, 1 \mathrm{H}), 3.98(\mathrm{t}, J=10.4 \mathrm{~Hz}, 1 \mathrm{H}), 3.64(\mathrm{ddd}, J=10.2,9.1,4.8 \mathrm{~Hz}, 1 \mathrm{H}), 1.12(\mathrm{~s}, 9 \mathrm{H}), 1.12(\mathrm{~s}, 9 \mathrm{H}) .{ }^{13} \mathrm{C}\left\{{ }^{1} \mathrm{H}\right\}$ NMR $\left(101 \mathrm{MHz}, \mathrm{CDCl}_{3}\right) \delta 139.7,134.0,128.6,128.2,80.0,71.5,68.6,27.5,27.2,22.8,20.3$. IR 2934, 2860, 1455, $827 \mathrm{~cm}^{-1}$; HRMS (ESI) m/z: [M + Na $]$ calculated for C17H27CINaO3Si 365.1310 Found 365.1341 . 
<smiles>CC(C)(C)[Si]1(C(C)(C)C)OC[C@@H](O)C(c2cccc(Br)c2)O1</smiles>

Compound 45: Synthesized using Procedure E; Purified using a gradient of 0 to $20 \%$ EtOAc/Hexanes on silica gel; (colorless foam, $66 \mathrm{mg}, 85 \%$ yield); ${ }^{1} \mathrm{H}$ NMR $\left(600 \mathrm{MHz}, \mathrm{CDCl}_{3}\right) \delta 7.64(\mathrm{t}, J=1.8 \mathrm{~Hz}, 1 \mathrm{H}), 7.50$ (ddd, $J=7.9,2.1,1.1 \mathrm{~Hz}, 1 \mathrm{H}), 7.42(\mathrm{dd}, J=7.8,1.5 \mathrm{~Hz}, 1 \mathrm{H}), 7.30(\mathrm{t}, J=7.8 \mathrm{~Hz}, 1 \mathrm{H}), 4.78(\mathrm{~d}, J=9.1 \mathrm{~Hz}, 1 \mathrm{H})$, $4.23(\mathrm{dd}, J=10.6,4.8 \mathrm{~Hz}, 1 \mathrm{H}), 3.98(\mathrm{t}, J=10.4 \mathrm{~Hz}, 1 \mathrm{H}), 3.71-3.57(\mathrm{~m}, 1 \mathrm{H}), 1.13(\mathrm{~s}, 9 \mathrm{H}), 1.12(\mathrm{~s}, 9 \mathrm{H})$. ${ }^{13} \mathrm{C}\left\{{ }^{1} \mathrm{H}\right\}$ NMR $\left(101 \mathrm{MHz}, \mathrm{CDCl}_{3}\right) \delta 143.6,131.2,130.1,130.0,125.5,122.6,79.9,71.4,68.6,27.5,27.2$, 22.8, 20.3. IR 2934, 2860, 1541, 1473, 1061, $827 \mathrm{~cm}^{-1}$.; HRMS (ESI) m/z: [M + Na ${ }^{+}$calculated for C17H27BrNaO3Si 409.0805, Found 409.0799.

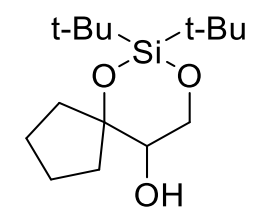

7,7-di-tert-butyl-6,8-dioxa-7-silaspiro[4.5]decan-10-ol

Compound 46: Synthesized using Procedure E; Purified using a gradient of 0 to 20\% EtOAc/hexanes on silica gel; (colorless oil, $31 \mathrm{mg}, 54 \%$ yield); ${ }^{1} \mathrm{H} \mathrm{NMR}\left(400 \mathrm{MHz}, \mathrm{CDCl}_{3}\right) \delta 4.10$ (dd, J = 11.1, $3.0 \mathrm{~Hz}, 1 \mathrm{H}$ ), 3.93 (dd, $J=11.0,7.5 \mathrm{~Hz}, 1 \mathrm{H}), 3.76(\mathrm{dd}, J=7.6,2.9 \mathrm{~Hz}, 1 \mathrm{H}), 1.97-1.72(\mathrm{~m}, 6 \mathrm{H}), 1.68-1.59(\mathrm{~m}, 2 \mathrm{H}), 1.06(\mathrm{~s}$, 9H), 1.04 (s, 9H). $\left.{ }^{13} \mathrm{C} \mathrm{NMR} \mathrm{(101} \mathrm{MHz,} \mathrm{CDCl}_{3}\right) \delta 87.5,72.2,66.2,39.3,34.6,27.6,27.4,23.9,23.8,21.2$, 21.0. IR 3442, 2963, 2860, 1473, 1061, $822 \mathrm{~cm}^{-1}$; HRMS (ESI) m/z: [M + Na+] calculated for C15H30NaO3Si 309.1856 Found 309.1867.<smiles>CC(C)(C)[Si]1(C(C)(C)C)OCC(O)C2(CCCCC2)O1</smiles>

2,2-di-tert-butyl-1,3-dioxa-2-silaspiro[5.5]undecan-5-ol

Compound 47: Synthesized using Procedure E; Purified using a gradient of 0 to $20 \%$ EtOAc/Hexanes on silica gel; (white solid, $37 \mathrm{mg}, 60 \%$ yield); ${ }^{1} \mathrm{H} \mathrm{NMR}\left(400 \mathrm{MHz}, \mathrm{CDCl}_{3}\right) \delta 4.08-3.86(\mathrm{~m}, 2 \mathrm{H}), 3.62$ (dd, $J=$ 7.7, 3.7 Hz, 1H), $1.91-1.40(\mathrm{~m}, 10 \mathrm{H}), 1.07(\mathrm{~s}, 9 \mathrm{H}), 1.04(\mathrm{~s}, 9 \mathrm{H}) .{ }^{13} \mathrm{C} \mathrm{NMR}\left(101 \mathrm{MHz}, \mathrm{CDCl}_{3}\right) \delta$ 76.4, 74.3, $64.3,37.0,30.8,27.7,27.5,25.9,21.3,21.2,21.0,20.9$. IR 3460, 2934, 2849, 1473, 1056, $827 \mathrm{~cm}^{-1}$.; HRMS (ESI) m/z: [M + $\left.\mathrm{Na}^{+}\right]$calculated for C16H32NaO3Si 323.2013, Found 323.2036. 


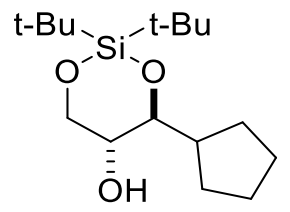

2,2-di-tert-butyl-4-cyclopentyl-1,3,2-dioxasilinan-5-ol

Compound 48: Synthesized using Procedures E $(0.132 \mathrm{mmol}$ scale) and $\mathrm{F}(0.2 \mathrm{mmol}$ scale); purified using a gradient of 0 to $30 \%$ Acetone/DCM on silica gel after Procedure E; single diastereomer; (colorless oil, $17 \mathrm{mg}, 43 \%$ yield); ${ }^{1} \mathrm{H} \mathrm{NMR}\left(400 \mathrm{MHz}, \mathrm{CDCl}_{3}\right) \delta 4.15$ (ddt, $\left.J=10.5,4.6,1.5 \mathrm{~Hz}, 1 \mathrm{H}\right), 3.89$ (ddt, $J=8.9,3.4$, $1.6 \mathrm{~Hz}, 1 \mathrm{H}), 3.85-3.74(\mathrm{~m}, 1 \mathrm{H}), 3.74-3.61(\mathrm{~m}, 1 \mathrm{H}), 2.38-2.25(\mathrm{~m}, 1 \mathrm{H}), 1.65(\mathrm{~m}, 8 \mathrm{H}), 1.14-1.04(\mathrm{~m}$, 9H), $1.04-0.88(\mathrm{~m}, 9 \mathrm{H}) .{ }^{13} \mathrm{C}\left\{{ }^{1} \mathrm{H}\right\} \mathrm{NMR}\left(101 \mathrm{MHz}, \mathrm{CDCl}_{3}\right) \delta 80.0,69.6,69.0,41.5,28.6,27.6,27.1,26.0$, 25.9, 25.1, 22.7, 20.3. IR 3340, 2940, 2849, 1473, $1044 \mathrm{~cm}^{-1}$; HRMS (ESI) m/z: [M + $\left.\mathrm{Na}^{+}\right]$) calculated for C16H32NaO3Si 323.2013 Found 323.1985.<smiles>CC(C)[Si](O)(OC(C)(C)C)C(C)(C)C</smiles>

di-tert-butyl(tetrahydrofuran-2-yl)ethoxy)silanol, erythro isomer

Compound 50: Synthesized using Procedure B on a 0.2 and $1 \mathrm{mmol} \mathrm{scale;} \mathrm{Purified} \mathrm{using} \mathrm{a} \mathrm{gradient} \mathrm{of} 0$ to 2\% acetone/DCM on silica gel; single diastereomer; (colorless oil, $0.2 \mathrm{mmol}$ scale: $36 \mathrm{mg} ; 1 \mathrm{mmol}$ scale: $178 \mathrm{mg}, 65 \%$ yield); ${ }^{1} \mathrm{H} \mathrm{NMR}\left(400 \mathrm{MHz}, \mathrm{CDCl}_{3}\right) \delta 4.41$ (dd, $\left.J=6.5,2.5 \mathrm{~Hz}, 1 \mathrm{H}\right), 3.97-3.91(\mathrm{~m}, 1 \mathrm{H}), 3.79-$ $3.71(\mathrm{~m}, 1 \mathrm{H}), 3.65(\mathrm{ddd}, J=8.5,4.7,2.7 \mathrm{~Hz}, 1 \mathrm{H}), 1.98-1.88(\mathrm{~m}, 2 \mathrm{H}), 1.93-1.84(\mathrm{~m}, 1 \mathrm{H}), 1.75(\mathrm{dd}, J=5.4$, $2.4 \mathrm{~Hz}, 1 \mathrm{H}), 1.15(\mathrm{~d}, J=6.5 \mathrm{~Hz}, 3 \mathrm{H}), 1.04(\mathrm{~s}, 9 \mathrm{H}), 0.99(\mathrm{~s}, 9 \mathrm{H}) . ;{ }^{13} \mathrm{C}\left\{{ }^{1} \mathrm{H}\right\} \mathrm{NMR}\left(101 \mathrm{MHz}, \mathrm{CDCl}_{3}\right) \delta 84.2,68.1$, 67.7, 27.9, 27.7, 25.2, 23.2, 21.2, 20.5, 20.3; IR: 3436, 2963, 2860, 1475, $650.9 \mathrm{~cm}^{-1}$.; HRMS (ESI) m/z: [M $\left.+\mathrm{Na}^{+}\right]$calculated for $\mathrm{C}_{14} \mathrm{H}_{30} \mathrm{O}_{3} \mathrm{SiNa}^{+} 297.1862$, Found 297.1881.<smiles>CC(O[Si](C)(C)C)[C@H]1CCCO1</smiles>

di-tert-butyl(tetrahydrofuran-2-yl)ethoxy)silanol, threo isomer

Compound 52: Synthesized using Procedure B on a $0.2 \mathrm{mmol}$ scale; Purified using a gradient of 0 to $2 \%$ acetone/DCM on silica gel; single diastereomer; (colorless oil, $36 \mathrm{mg}, 65 \%$ yield); ${ }^{1} \mathrm{H} \mathrm{NMR}\left(600 \mathrm{MHz}, \mathrm{CDCl}_{3}\right.$ ) $\delta 3.91(\mathrm{dq}, J=8.0,6.3 \mathrm{~Hz}, 1 \mathrm{H}), 3.87-3.76(\mathrm{~m}, 2 \mathrm{H}), 3.65-3.58(\mathrm{~m}, 1 \mathrm{H}), 1.96-1.81(\mathrm{~m}, 3 \mathrm{H}), 1.43-1.33$ $(\mathrm{m}, 1 \mathrm{H}), 1.12(\mathrm{~d}, J=6.3 \mathrm{~Hz}, 3 \mathrm{H}), 1.02(\mathrm{~s}, 9 \mathrm{H}), 0.98(\mathrm{~s}, 9 \mathrm{H}) . ;{ }^{13} \mathrm{C}\left\{{ }^{1} \mathrm{H}\right\} \mathrm{NMR}\left(101 \mathrm{MHz}, \mathrm{CDCl}_{3}\right) \delta$ 85.4, 72.5, 67.9, 28.5, 27.7, 27.4, 26.1, 21.1, 20.3, 20.2.; IR: 3465, 2963, 2860, 1102, $651 \mathrm{~cm}^{-1}$.; HRMS (ESI) m/z: [M + Na+] calculated for $\mathrm{C}_{14} \mathrm{H}_{30} \mathrm{O}_{3} \mathrm{SiNa} 297.1862$, Found 297.1863 . 


\section{Structural Proof}

As we assembled the substrate scope for this project, a constant issue which plagued characterization was the possibility of either exo or endo opening of the epoxide in the cyclization reaction. In this section, for each class of epoxy-silanol, we present our reasoning for our structural assignments.

Structural Class I: silanol epoxides derived from trans-allylic silanols

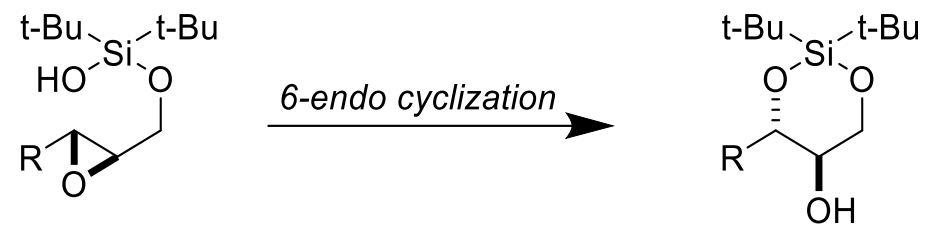

Reasoning: Crystal structure of $\mathbf{4 1}$. See section VII. Others were assigned by analogy.

Structural Class II: silanol epoxides derived from trans-homoallylic silanols

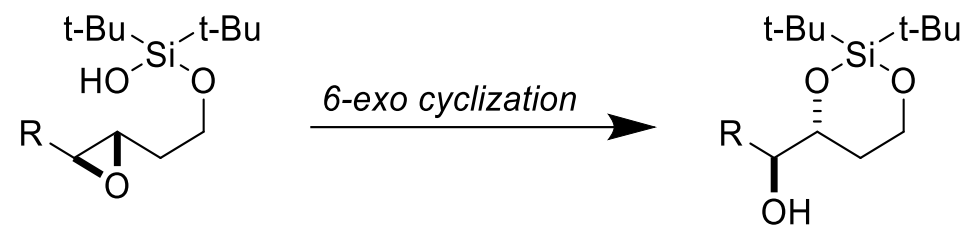

Reasoning: We prepared the acetate of $\mathbf{3 7}$<smiles>CC[C@H](O)C1CCO[Si](C(C)(C)C)(C(C)(C)C)O1</smiles>

37

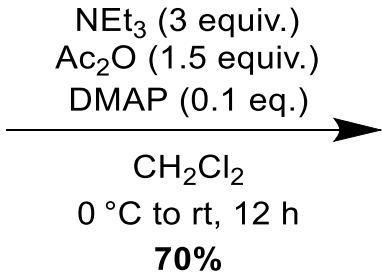<smiles>CC[C@H](OC(C)=O)C1CCO[Si](CC(C)(C)C)(C(C)(C)C)O1</smiles>

S1

A $10 \mathrm{~mL}$ round-bottom flask was charged with a stir-bar, 37 (17 mg, $0.062 \mathrm{mmol}, 1$ equivalent) and $2 \mathrm{~mL}$ of $\mathrm{CH}_{2} \mathrm{Cl}_{2}$. The reaction flask was cooled to $0{ }^{\circ} \mathrm{C}$ using an ice-water bath. Subsequently, $\mathrm{NEt}_{3}(26 \mu \mathrm{L}, 0.186$ mmol, 3 equiv.), $\mathrm{Ac}_{2} \mathrm{O}$ (9 $\mu \mathrm{L}, 0.093 \mathrm{mmol}, 1.5$ equiv.), and DMAP (1 mg, cat.) were added. The reaction mixture was allowed to warm to room temperature over a period of $12 \mathrm{~h}$. Then, the reaction was quenched with $4 \mathrm{~mL}$ of saturated aqueous $\mathrm{NaHCO}_{3}$ solution. The mixture was transferred to a separatory funnel, the organic layer was removed, and then the aqueous layer was extracted with $\mathrm{CH}_{2} \mathrm{Cl}_{2}(2 \times 5 \mathrm{~mL})$. The combined organic layers were washed with $10 \mathrm{~mL}$ of saturated aqueous $\mathrm{NaCl}$ solution (brine), dried over anhydrous $\mathrm{Na}_{2} \mathrm{SO}_{4}$, and the solvent was removed in vacuo. The resulting residue was purified through silica gel column chromatography (hexane/ethyl acetate $=80: 20)$ to afford $14 \mathrm{mg}(0.044 \mathrm{mmol}, 70 \%)$ of S1 as a colorless oil.

S1 (1-(2,2-di-tert-butyl-1,3,2-dioxasilinan-4-yl)propyl acetate): $\left.{ }^{1} \mathrm{H} \mathrm{NMR} \mathrm{(400} \mathrm{MHz,} \mathrm{CDCl}_{3}\right) \delta 4.78$ (dddd, $J=$ 7.8, 5.8, 3.9, $1.3 \mathrm{~Hz}, 1 \mathrm{H}), 4.20-4.02(\mathrm{~m}, 3 \mathrm{H}), 2.06(\mathrm{~d}, J=1.3 \mathrm{~Hz}, 3 \mathrm{H}), 1.85$ (ddd, $J=12.7,5.0,2.7 \mathrm{~Hz}, 1 \mathrm{H}$ ), $1.79-1.68(\mathrm{~m}, 1 \mathrm{H}), 1.69-1.53(\mathrm{~m}, 2 \mathrm{H}), 1.02(\mathrm{~s}, 9 \mathrm{H}), 0.98(\mathrm{~s}, 9 \mathrm{H}), 0.92(\mathrm{td}, J=7.5,1.4 \mathrm{~Hz}, 3 \mathrm{H}) . ;{ }^{13} \mathrm{C}\left\{{ }^{1} \mathrm{H}\right\}$ NMR $\left(101 \mathrm{MHz}, \mathrm{CDCl}_{3}\right) \delta 170.8,78.1,74.4,64.3,32.3,27.5,27.2,23.2,22.9,21.3,20.0,9.5 . ;$ IR: 2957, 2860, 1747, 1090, $650.9 \mathrm{~cm}^{-1}$; HRMS (ESI) m/z: [M + $\mathrm{H}^{+}$] calculated for $\mathrm{C}_{16} \mathrm{H}_{33} \mathrm{O}_{4} \mathrm{Si} 317.2148$, Found 317.2135 . 
The two structural possibilities are:

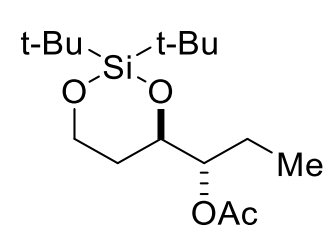

S1

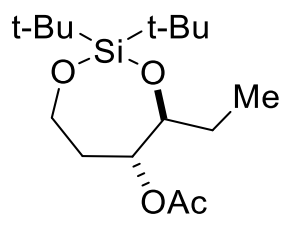

S2

There is a strong HMBC correlation between proton vicinal to the acetate group and the methyl group of the alkyl chain making S2 unlikely.

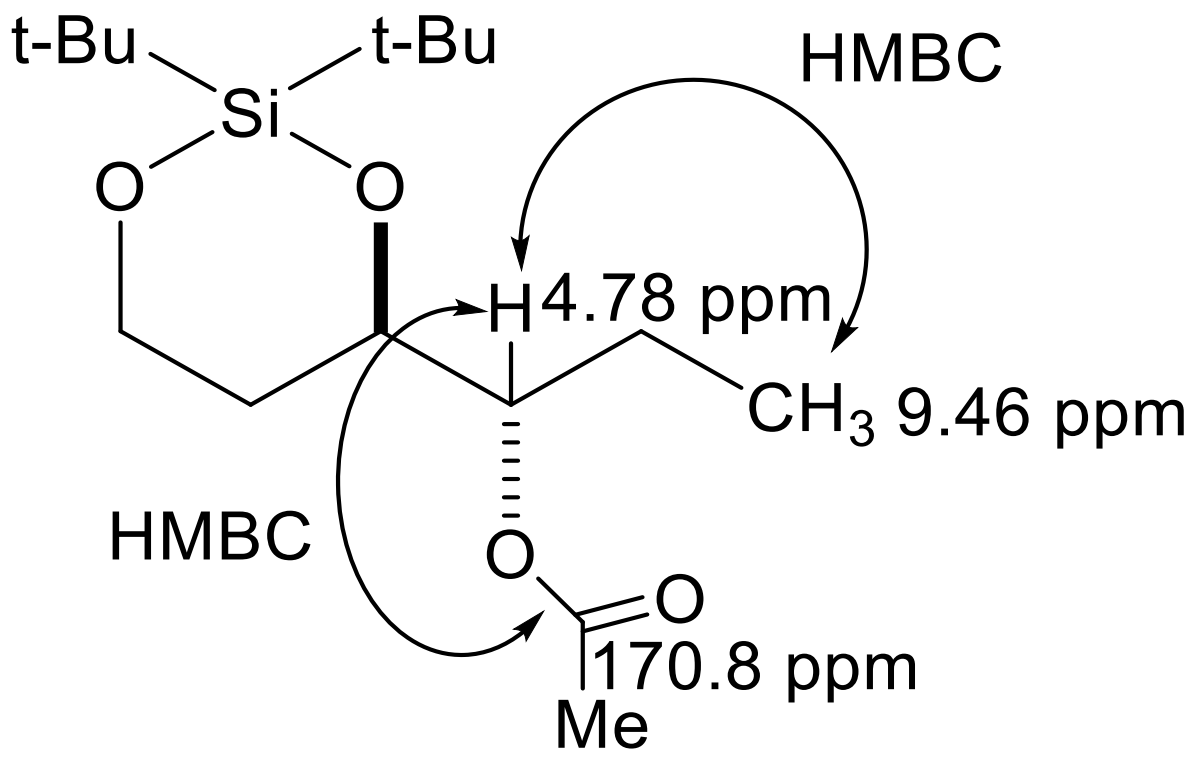


${ }^{1} \mathrm{H}$ NMR of $\mathbf{S 1}\left(400 \mathrm{MHz}, \mathrm{CDCl}_{3}\right)$

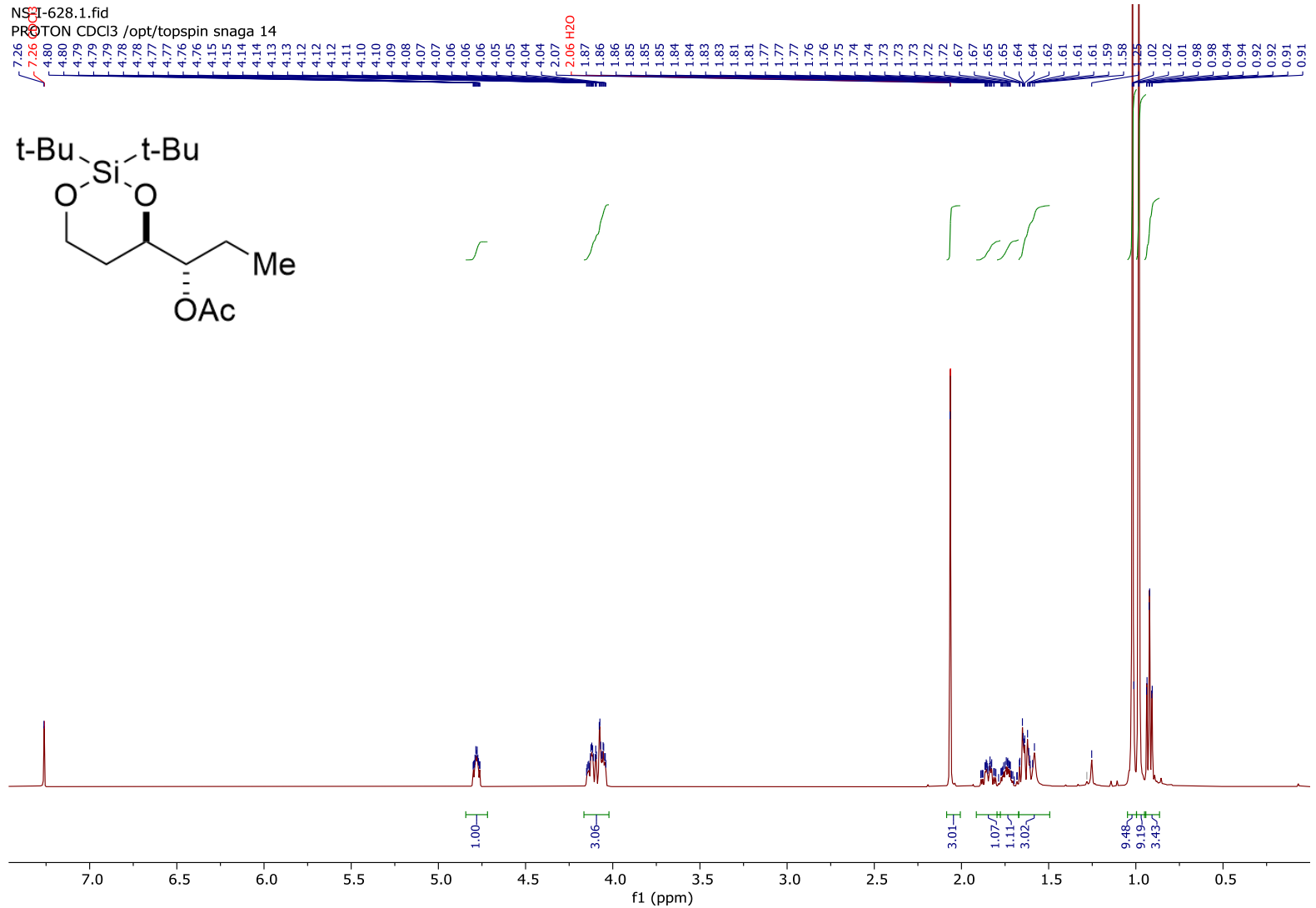

${ }^{13} \mathrm{C}\left\{{ }^{1} \mathrm{H}\right\}$ NMR of $\mathbf{S 1}\left(100 \mathrm{MHz}, \mathrm{CDCl}_{3}\right)$ NS-I-628.2.fid

C13CPD_SIENA CDCI3 /opt/topspin snaga 14 i<smiles>CC[C@H](OC(C)=O)C1CCO[Si](C(C)(C)C)(C(C)(C)C)O1</smiles>

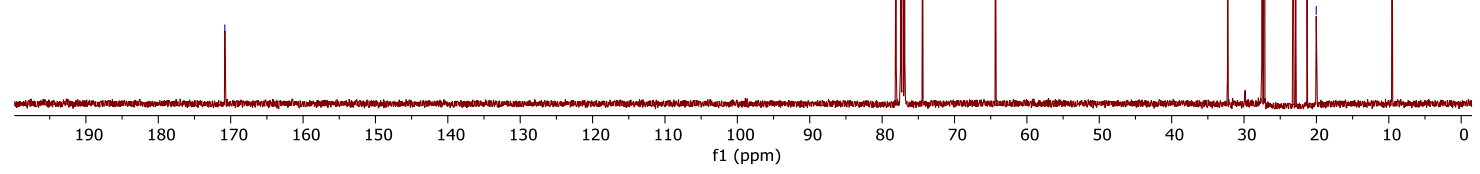


COSY of $\mathbf{S 1}\left(\mathrm{CDCl}_{3}\right)$

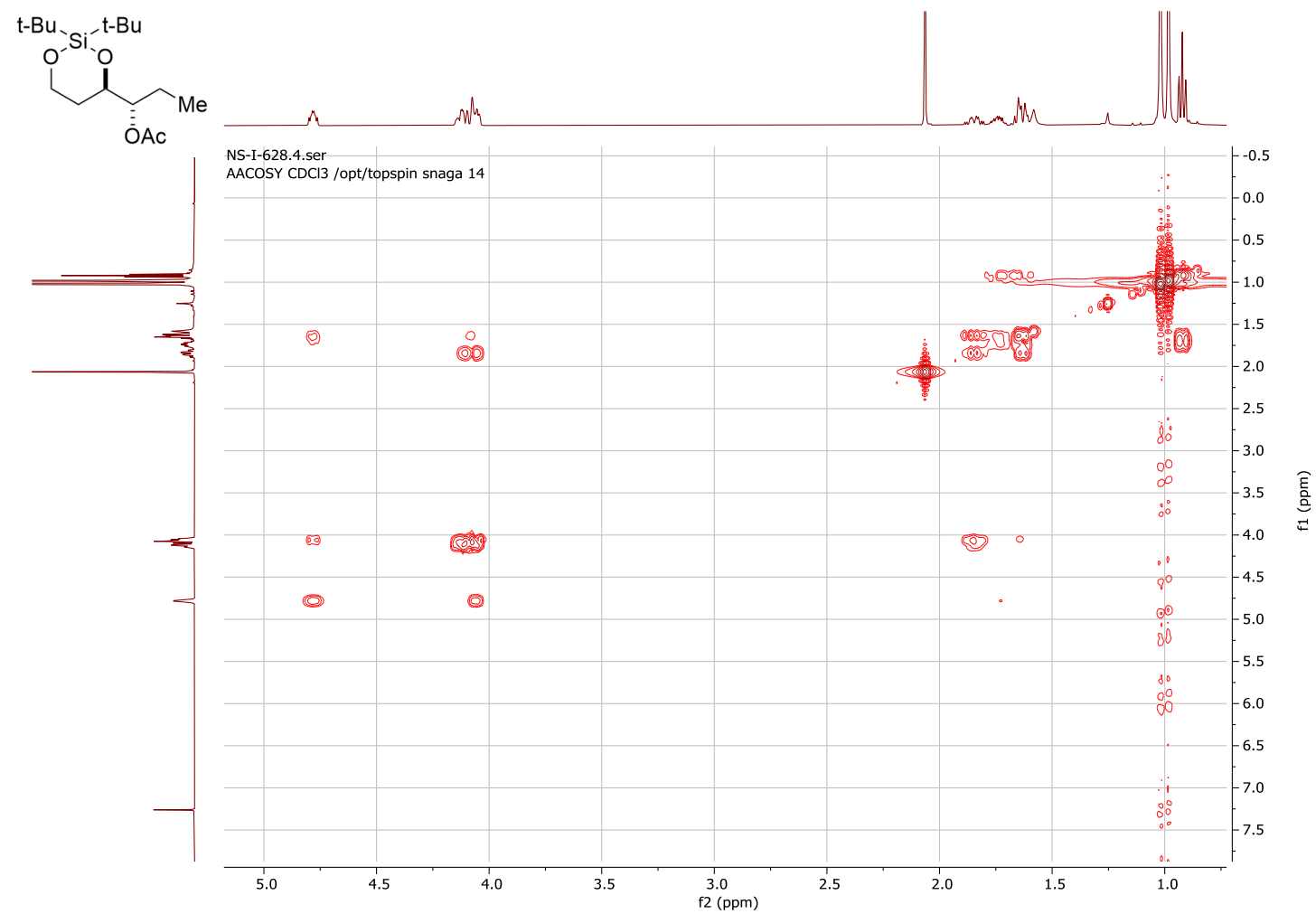

$\mathrm{HSQC}$ of $\mathrm{S} 1\left(\mathrm{CDCl}_{3}\right)$

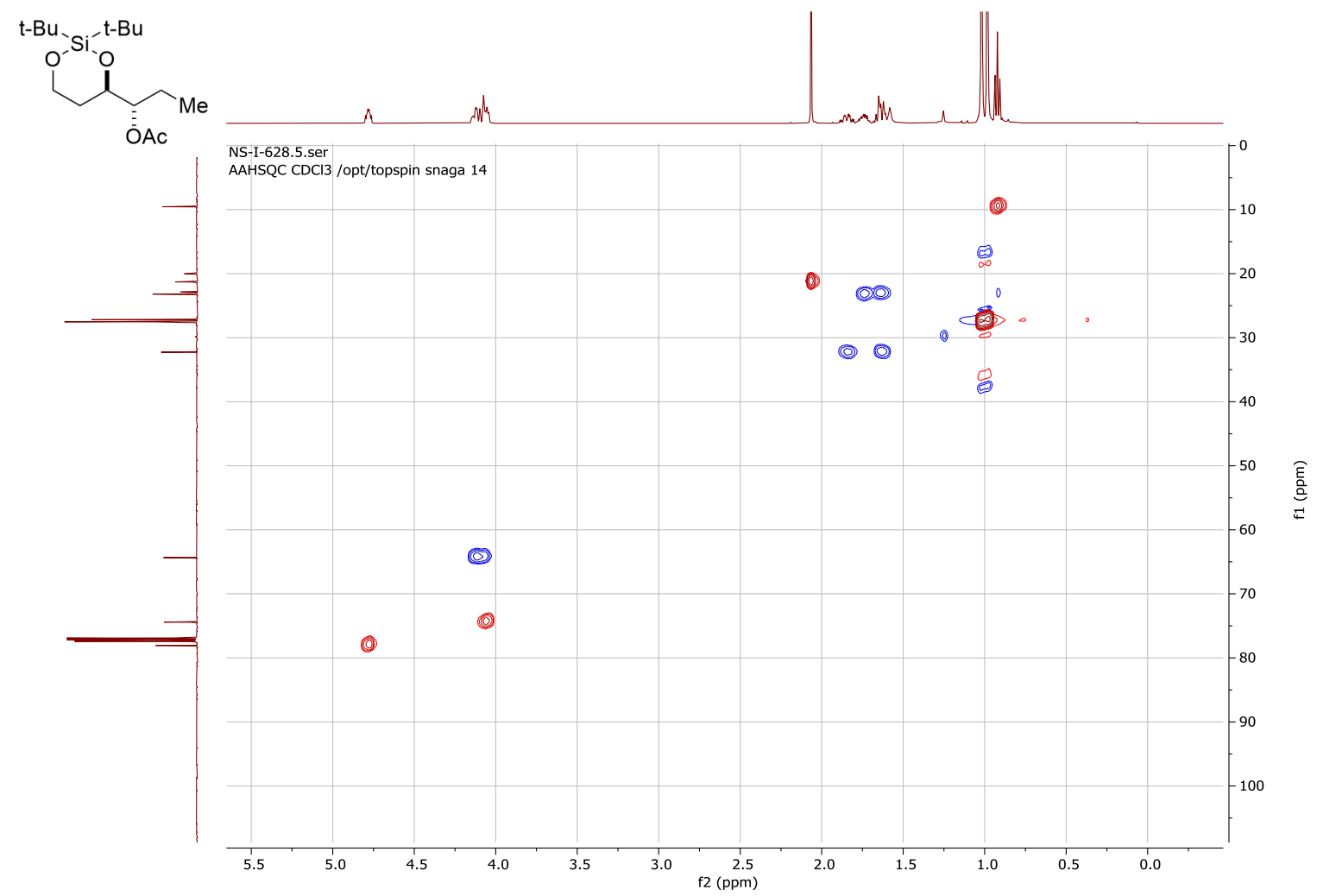




\section{$\mathrm{HMBC}$ of $\mathbf{S 1}\left(\mathrm{CDCl}_{3}\right)$}

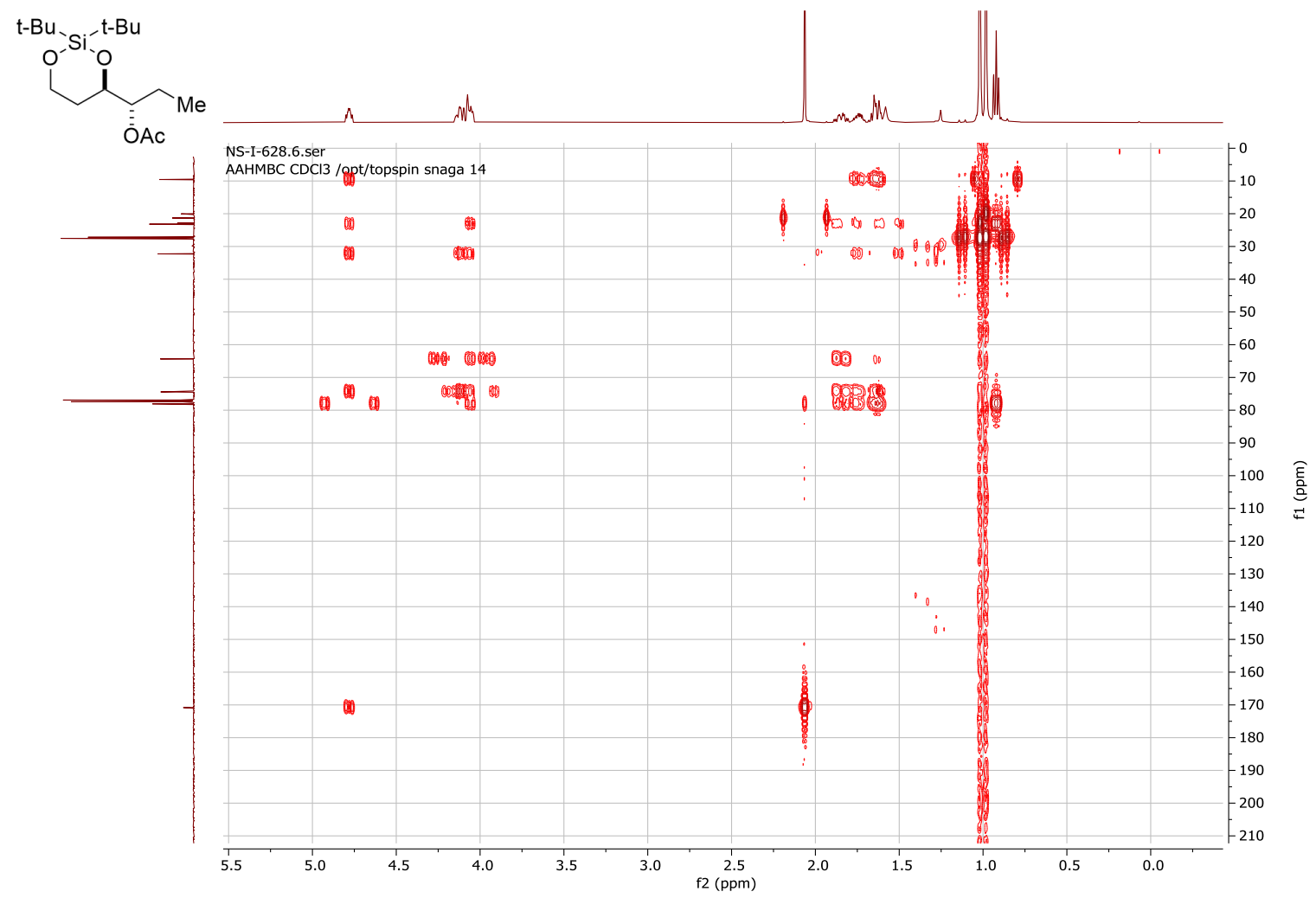

Structural Class III: silanol epoxides derived from cis-homoallylic silanols

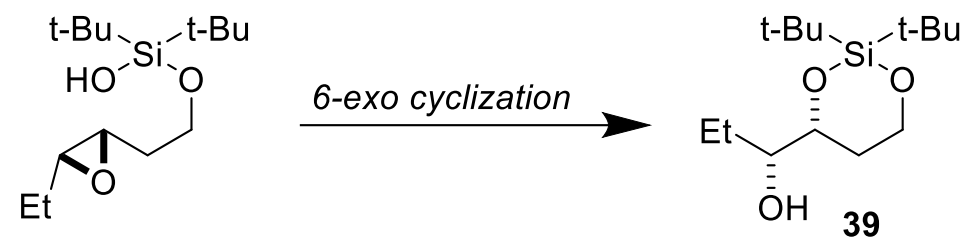

Reasoning: The two most reasonable structural possibilities are:<smiles>CC[C@H](O)[C@H]1CCO[Si](C(C)(C)C)(C(C)(C)C)O1</smiles>

39 vs.<smiles>CC[C@H]1O[Si](C(C)(C)C)(C(C)(C)C)OCC[C@H]1O</smiles>

S3

We are able to assign the proton vicinal to the hydroxyl group unambiguously via a COSY correlation. This proton has a strong $\mathrm{HMBC}$ correlation with the methyl group of the alkyl chain, making $\mathbf{3 9}$ likely. 


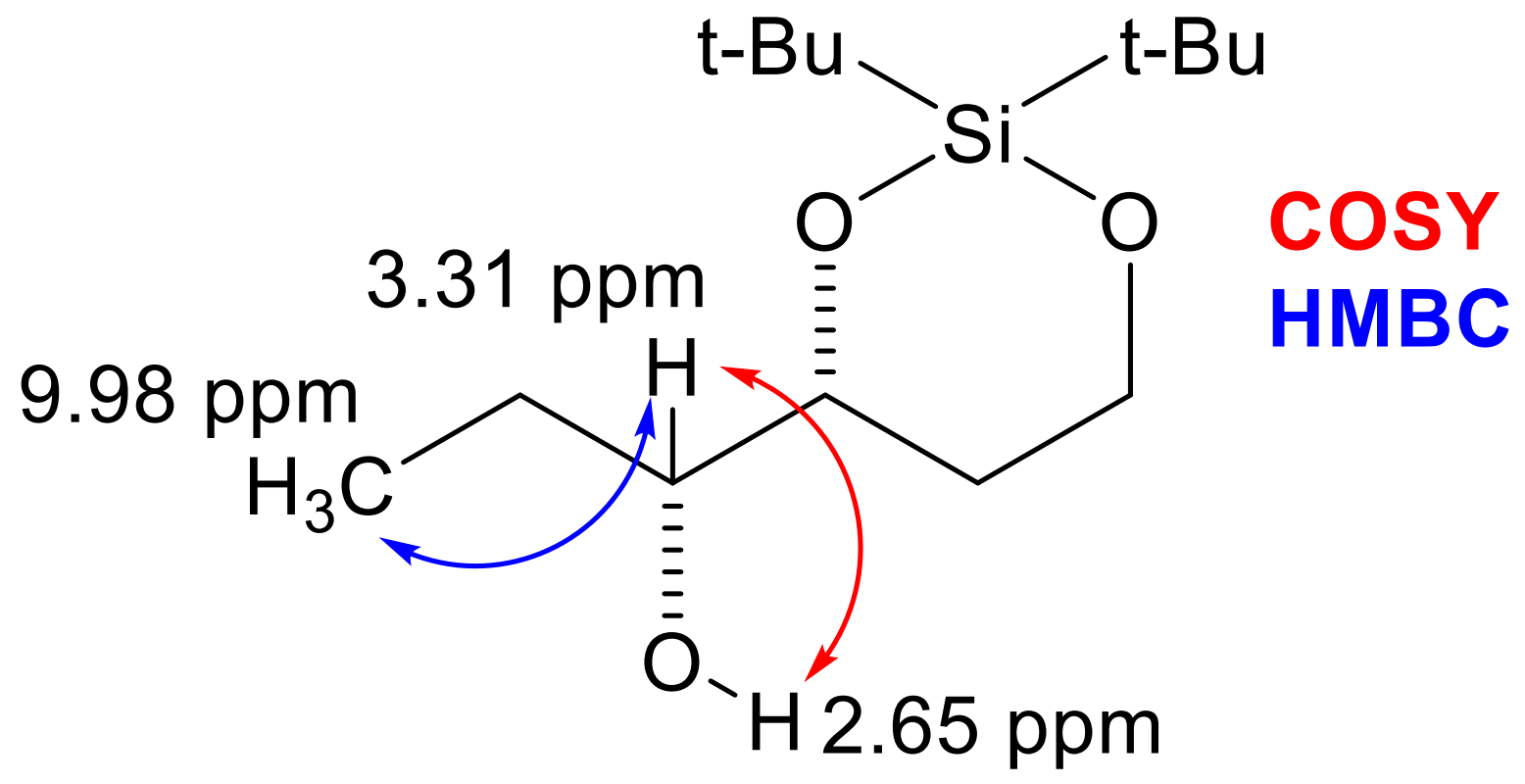

${ }^{1} \mathrm{H}$ NMR of $39\left(400 \mathrm{MHz}, \mathrm{CDCl}_{3}\right)$

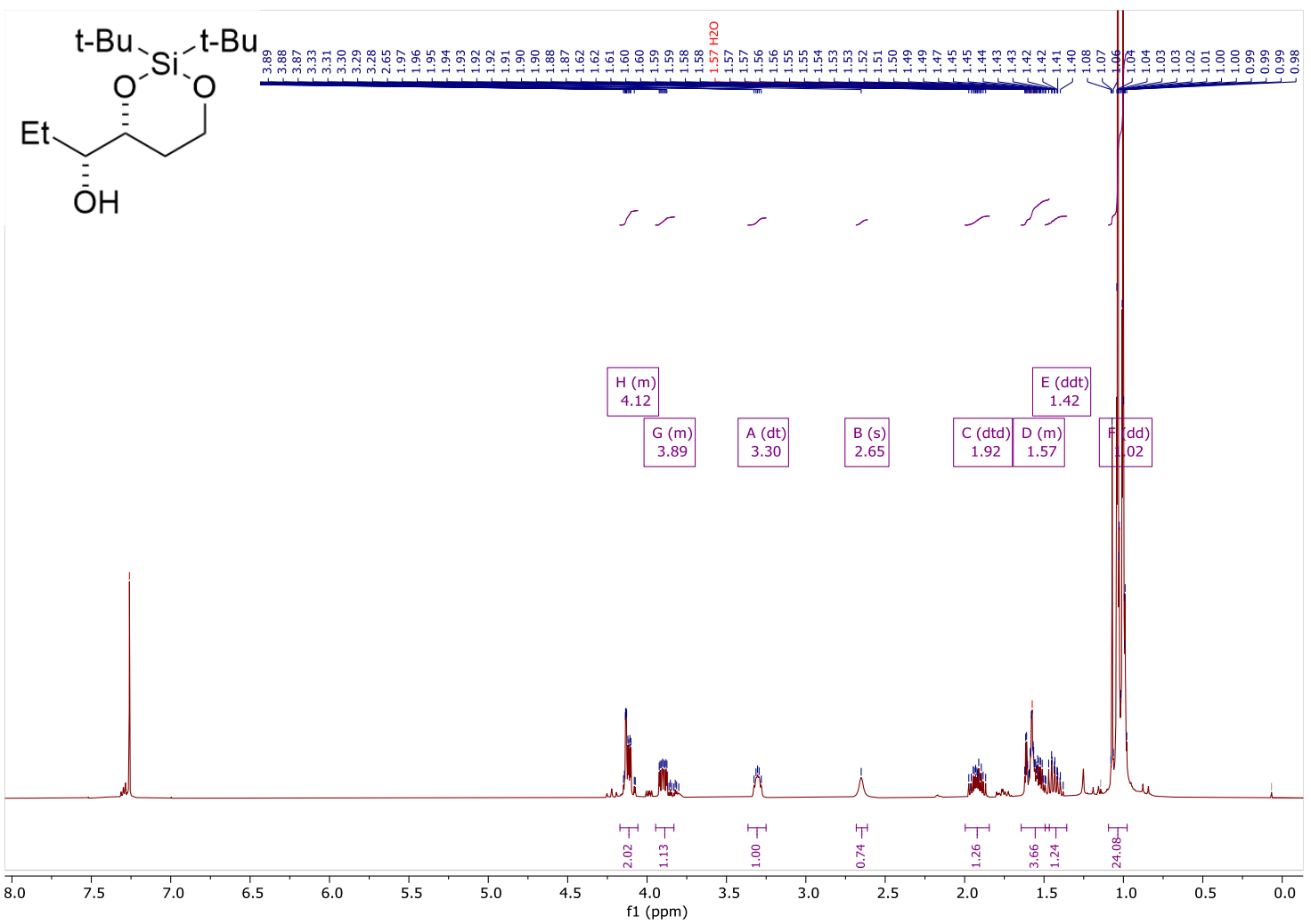


${ }^{13} \mathrm{C}$ NMR of $39\left(100 \mathrm{MHz}, \mathrm{CDCl}_{3}\right)$<smiles>CC[C@H](O)[C@H]1CCO[Si](C(C)(C)C)(C(C)(C)C)O1</smiles>

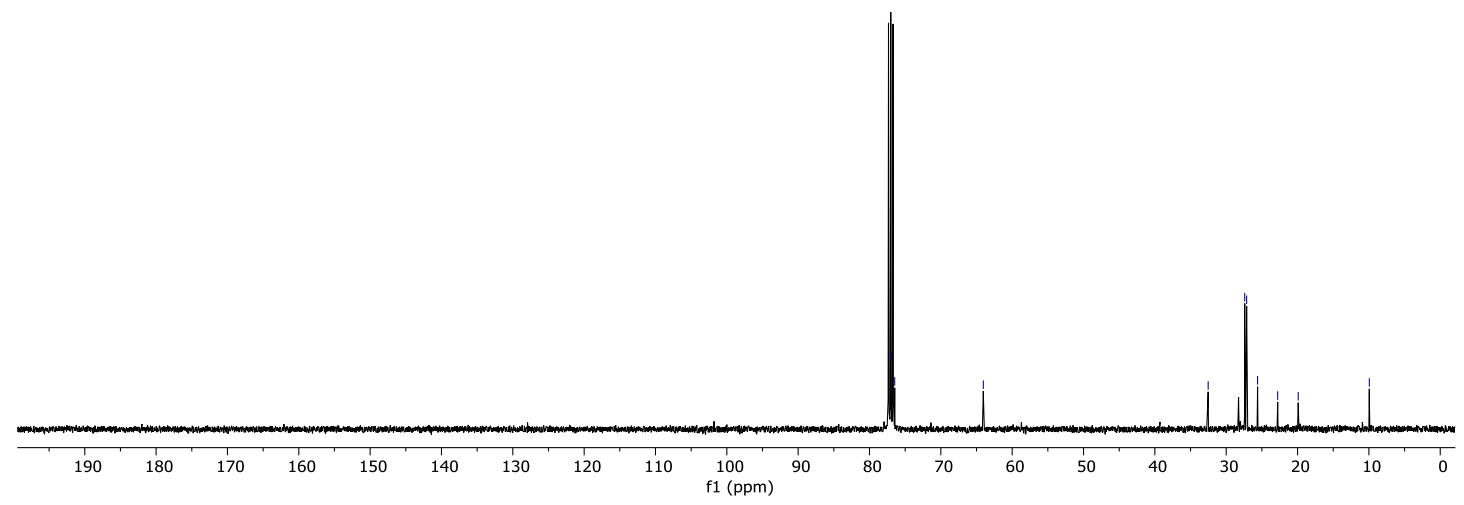

COSY of $39\left(\mathrm{CDCl}_{3}\right)$

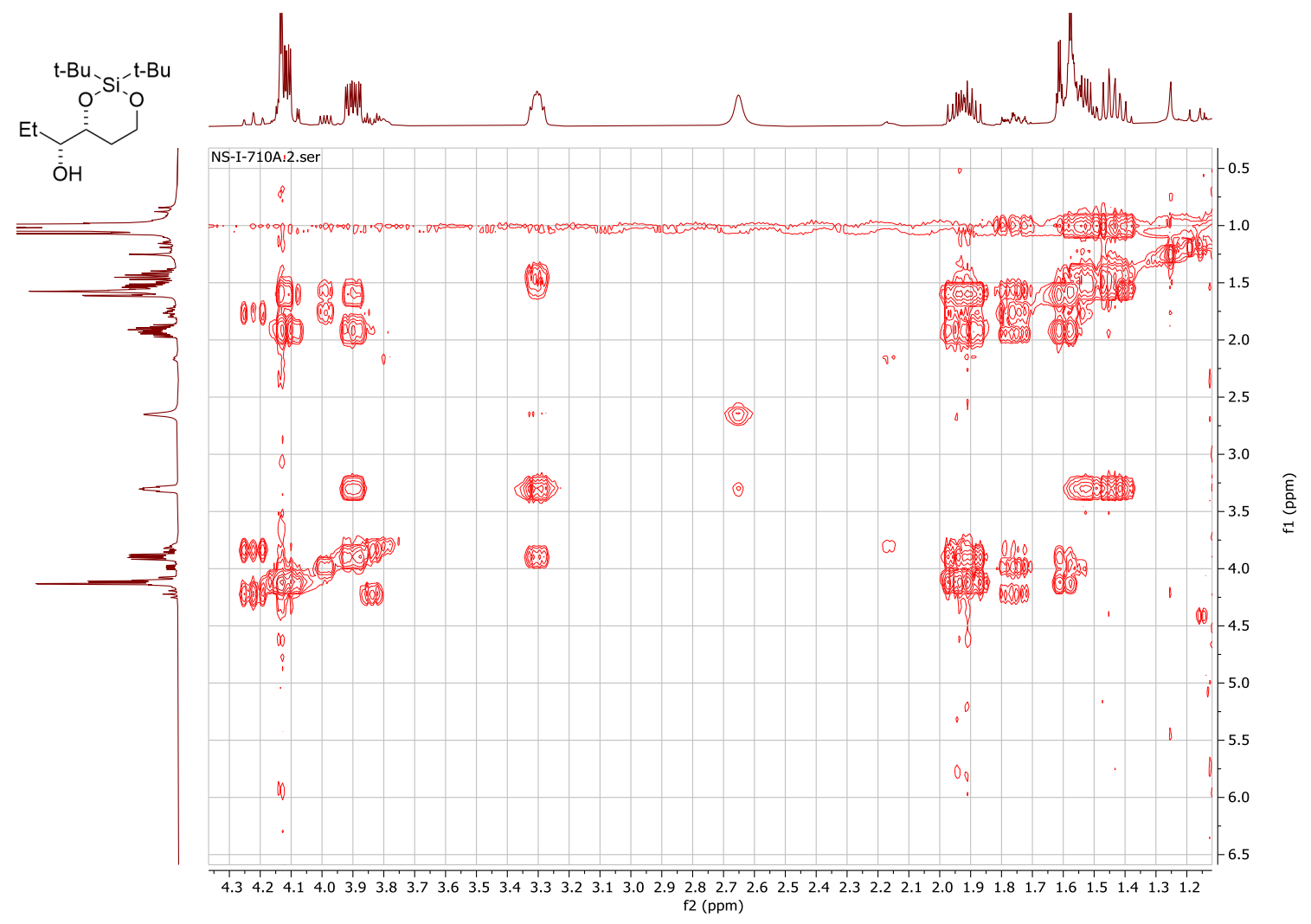



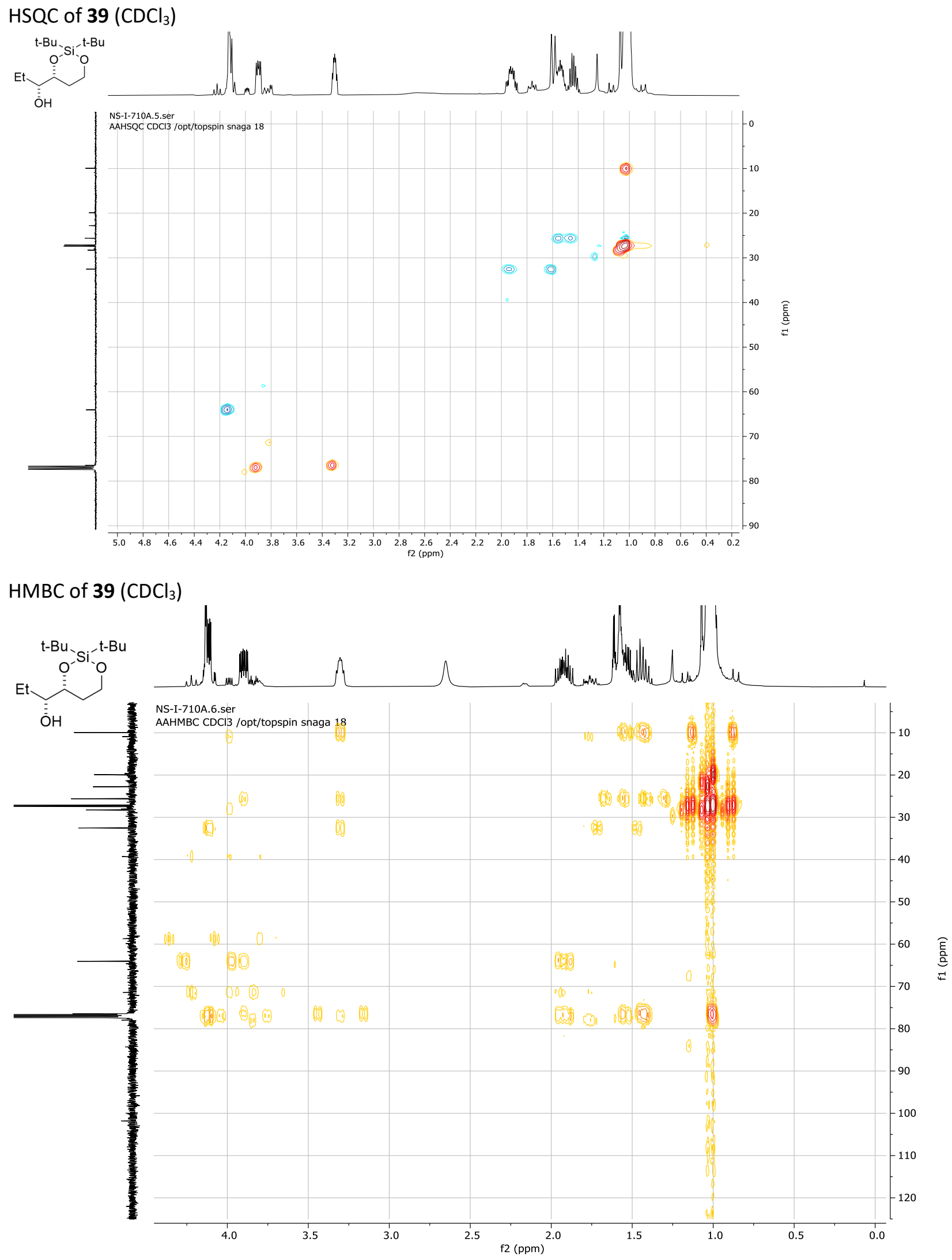
$\underline{\text { Structural Class IV: silanol epoxides derived from cis-allylic alcohols }}$

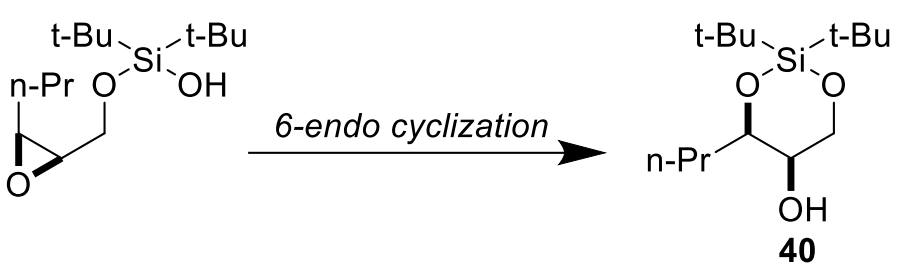

Reasoning: The two most likely structural possibilities are:<smiles>CCCCC(O)C1CO[Si](C(C)(C)C)(C(C)(C)C)OC1C(O)C(O)C1CO[Si](C(C)(C)C)(C(C)(C)C)O1</smiles>

We are able to assign the proton vicinal to the hydroxyl group unambiguously via a COSY correlation. In the COSY, this proton correlates with both sets of protons vicinal to the silanoxy group, making $\mathbf{4 0}$ likely.

\section{COSY correlations}

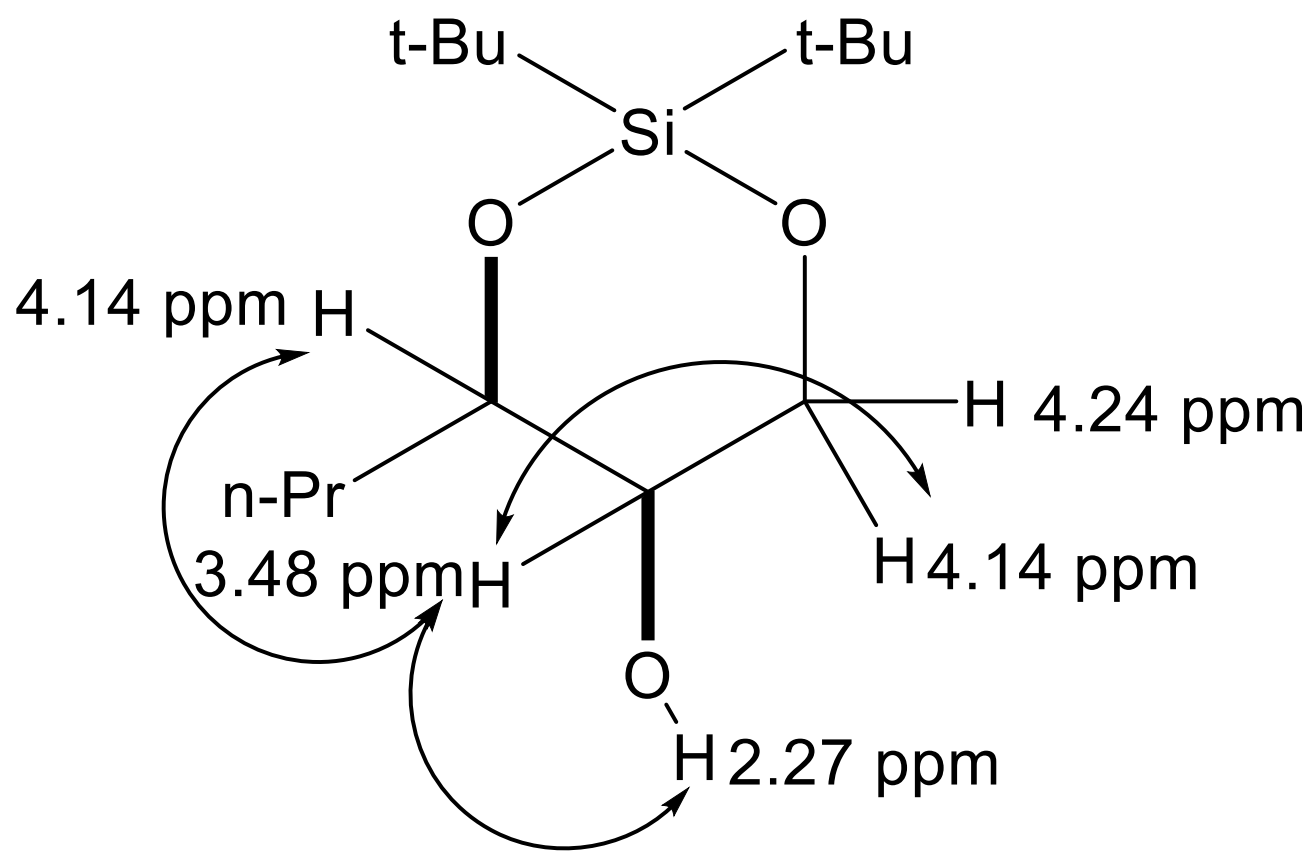


${ }^{1} \mathrm{H}$ NMR of $40\left(400 \mathrm{MHz}, \mathrm{CDCl}_{3}\right)$

NS-I I-12.1. fid

PROFON CDCl3 /opt/topspin snaga 53

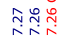

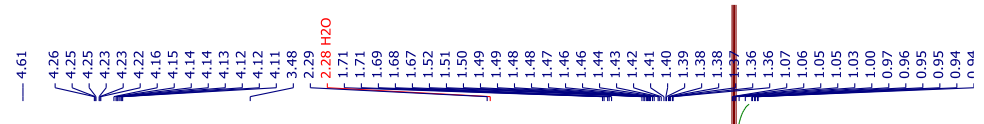

$\overbrace{\mathrm{OH}}^{\mathrm{t}-\mathrm{Bu}}$

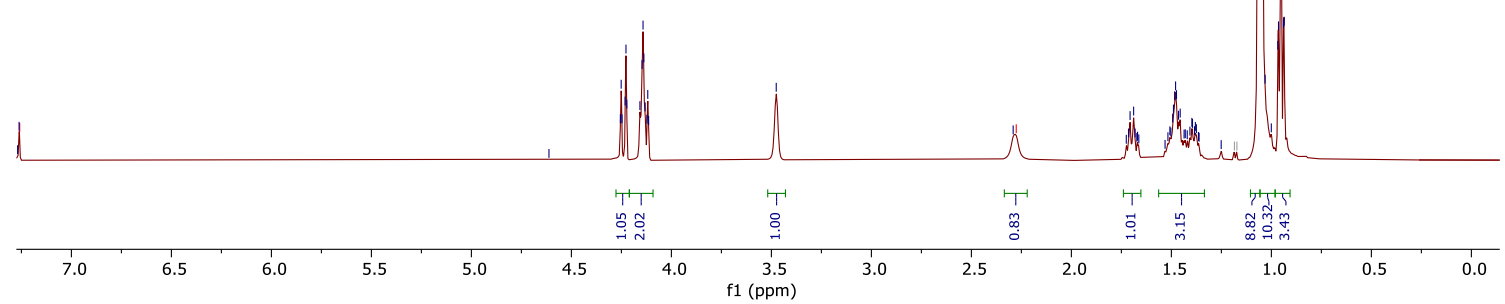

${ }^{13} \mathrm{C}$ NMR of $40\left(100 \mathrm{MHz}, \mathrm{CDCl}_{3}\right)$

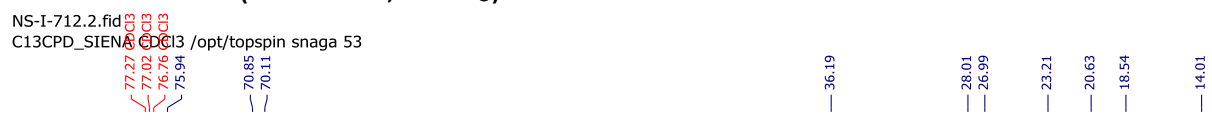<smiles>CCCCC(C)[Si]1(C(C)(C)C)OCC(O)C([13CH])O1</smiles>

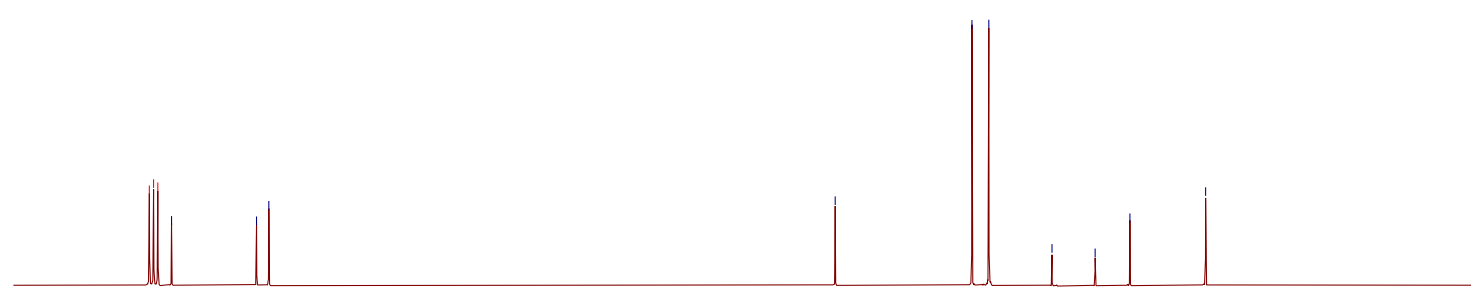

$\begin{array}{lllllllllllllllllll}75 & 80 & 75 & 70 & 65 & 60 & 55 & 50 & 45 & \begin{array}{r}10 \\ \mathrm{f} 1(\mathrm{ppm})\end{array} & 35 & 30 & 25 & 20 & 15 & 10 & 5 & 0\end{array}$ 


\section{COSY of $\mathbf{4 0}\left(\mathrm{CDCl}_{3}\right)$}

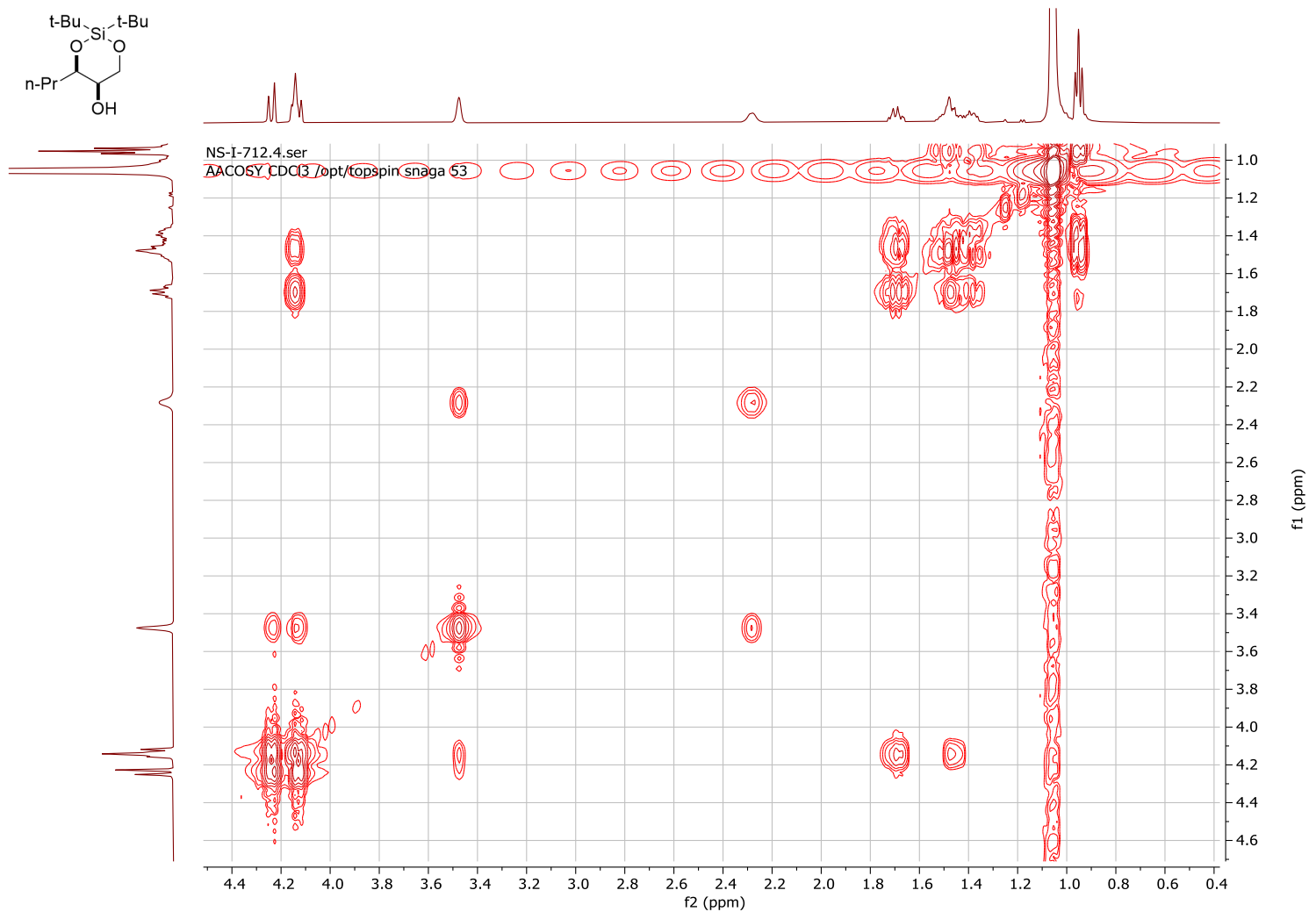

$\mathrm{HSQC}$ of $40\left(\mathrm{CDCl}_{3}\right)$

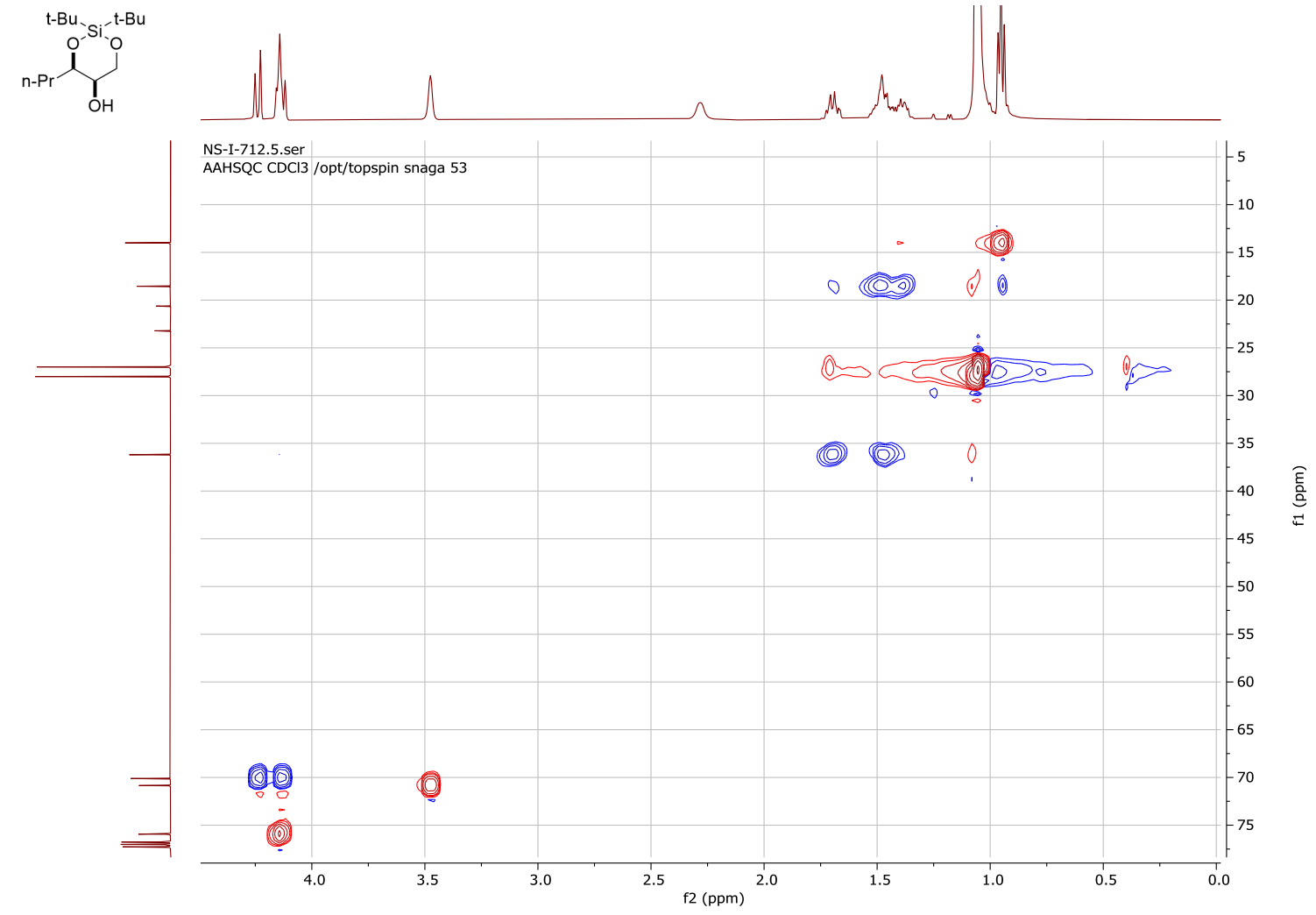


Structural Class V: silanol epoxides derived from 4-alkenyl silanols

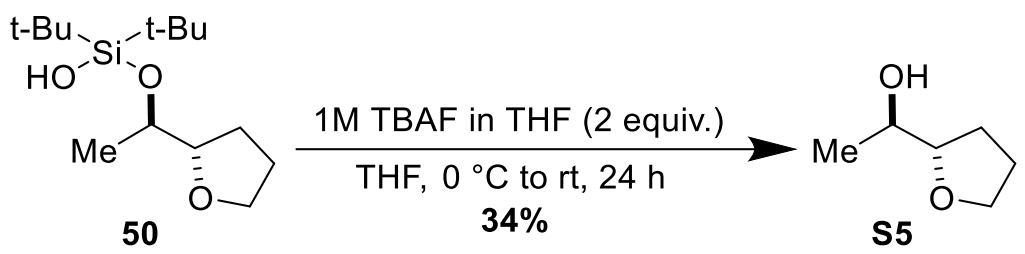

A $25 \mathrm{~mL}$ round-bottom flask was charged with a stir-bar, $\mathbf{5 0}(0.274 \mathrm{~g}, 1 \mathrm{mmol})$, and anhydrous THF $\left(5 \mathrm{~mL}\right.$ ). The reaction flask was cooled to $0^{\circ} \mathrm{C}$ using an ice-water bath. Subsequently, TBAF (1M solution in THF, $2 \mathrm{~mL}, 2 \mathrm{mmol}, 2$ equiv.) was added dropwise. The reaction mixture was allowed to warm to room temperature over a period of $24 \mathrm{~h}$. After this time, the reaction was diluted with EtOAc $(10 \mathrm{~mL})$, transferred to a separatory funnel, and washed once with saturated aqueous $\mathrm{NH}_{4} \mathrm{Cl}$ solution $(10 \mathrm{~mL})$. The organic layer was separated, and the aqueous layer was extracted with two additional portions of EtOAc $(2 \times 10 \mathrm{~mL})$. The combined organic layers were washed with brine solution $(15 \mathrm{~mL})$, dried over anhydrous $\mathrm{Na}_{2} \mathrm{SO}_{4}$, and the solvent was removed in vacuo. The resulting residue was purified through silica gel column chromatography (hexane/ethyl acetate $=30: 70)$ to afford $40 \mathrm{mg}(0.34 \mathrm{mmol}, 34 \%)$ of $\mathbf{S 5}$ as a colorless oil.

${ }^{1} \mathrm{H} \mathrm{NMR}\left(400 \mathrm{MHz}, \mathrm{CDCl}_{3}\right) \delta 3.99(\mathrm{qd}, J=6.5,3.5 \mathrm{~Hz}, 1 \mathrm{H}), 3.91(\mathrm{dt}, J=8.3,6.6 \mathrm{~Hz}, 1 \mathrm{H}), 3.85-3.74(\mathrm{~m}, 2 \mathrm{H})$, $2.05(\mathrm{~s}, 1 \mathrm{H}), 1.98-1.79(\mathrm{~m}, 4 \mathrm{H}), 1.16(\mathrm{~d}, J=6.5 \mathrm{~Hz}, 3 \mathrm{H}) . ;{ }^{13} \mathrm{C}\left\{{ }^{1} \mathrm{H}\right\} \mathrm{NMR}\left(101 \mathrm{MHz}, \mathrm{CDCl}_{3}\right) \delta 83.1,68.8,68.2$, 26.2, 24.6, 18.4. FT-IR: 3500, 2834, 1090, $690 \mathrm{~cm}^{-1}$. Matches what is reported in: Angew. Chem. Int. Ed. 2017, 56, 12619-12623.<smiles>CC(O)C1CCCO1</smiles>

S5

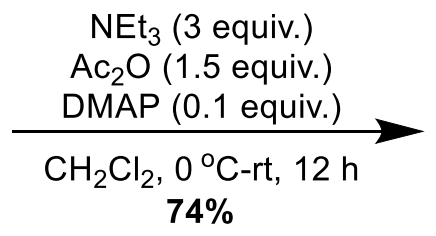

$74 \%$<smiles>CC(=O)OC(C)C1CCCO1</smiles>

s6

A $10 \mathrm{~mL}$ round-bottom flask was charged with a stir-bar, $\mathbf{S 5}$ (20 $\mathrm{mg}, 0.172 \mathrm{mmol})$, and $1 \mathrm{~mL}$ of anhydrous $\mathrm{CH}_{2} \mathrm{Cl}_{2}$. The reaction mixture was cooled to $0{ }^{\circ} \mathrm{C}$ using an ice-water bath. $\mathrm{NEt}_{3}(72 \mu \mathrm{L}, 0.52$ mmol, 3 equiv.), Ac ${ }_{2} \mathrm{O}(25 \mu \mathrm{L}, 0.26 \mathrm{mmol}, 1.5$ equiv.), and DMAP ( $1 \mathrm{mg}$, cat.) were subsequently added. The reaction mixture was warmed to room temperature over a period of $12 \mathrm{~h}$. Then, the reaction was quenched saturated $\mathrm{NaHCO}_{3}(4 \mathrm{~mL})$. The mixture was transferred to a separatory funnel, the organic layer was removed, and then the aqueous layer was extracted with $\mathrm{CH}_{2} \mathrm{Cl}_{2}(2 \times 5 \mathrm{~mL})$. The combined organic layers were washed with $10 \mathrm{~mL}$ of saturated aqueous $\mathrm{NaCl}$ solution (brine), dried over anhydrous $\mathrm{Na}_{2} \mathrm{SO}_{4}$, and the solvent was removed in vacuo. The resulting residue was purified through silica gel column chromatography (hexane/ethyl acetate $=70: 30)$ to afford $20 \mathrm{mg}(0.11 \mathrm{mmol}, 74 \%)$ of $\mathbf{S 6}$ as a colorless oil.

S6 is a known compound and has been previously characterized in the literature (J. Org. Chem. 2008, 73, 2270-2274.)

\begin{tabular}{|l|l|}
\hline Observed ${ }^{1} \mathrm{H}$ NMR shifts $\left(\mathrm{CDCl}_{3}, 400 \mathrm{MHz}\right)$ & Reported ${ }^{1} \mathrm{H}$ NMR shifts $\left(\mathrm{CDCl}_{3}, 300 \mathrm{MHz}\right)$ \\
\hline $4.92(\mathrm{dd}, J=6.4,4.8 \mathrm{~Hz}, 1 \mathrm{H})$ & $4.96-4.88(\mathrm{~m}, 1 \mathrm{H})$ \\
\hline $3.94-3.73(\mathrm{~m}, 3 \mathrm{H})$ & $3.93-3.74(\mathrm{~m}, 3 \mathrm{H})$ \\
\hline $2.05(\mathrm{~s}, 3 \mathrm{H})$ & $2.05(\mathrm{~s}, 3 \mathrm{H})$ \\
\hline
\end{tabular}




\begin{tabular}{|l|l|}
\hline $2.01-1.82(\mathrm{~m}, 3 \mathrm{H})$ & $1.97-1.84(\mathrm{~m}, 3 \mathrm{H})$ \\
\hline $1.74-1.64(\mathrm{~m}, 1 \mathrm{H})$ & $1.72-1.66(\mathrm{~m}, 1 \mathrm{H})$ \\
\hline $1.22(\mathrm{~d}, J=6.4 \mathrm{~Hz}, 3 \mathrm{H})$ & $1.23(\mathrm{~d}, J=6.4 \mathrm{~Hz}, 3 \mathrm{H})$ \\
\hline
\end{tabular}

${ }^{1} \mathrm{H}$ NMR spectrum of $\mathbf{S 6}\left(\mathrm{CDCl}_{3}, 400 \mathrm{MHz}\right)$

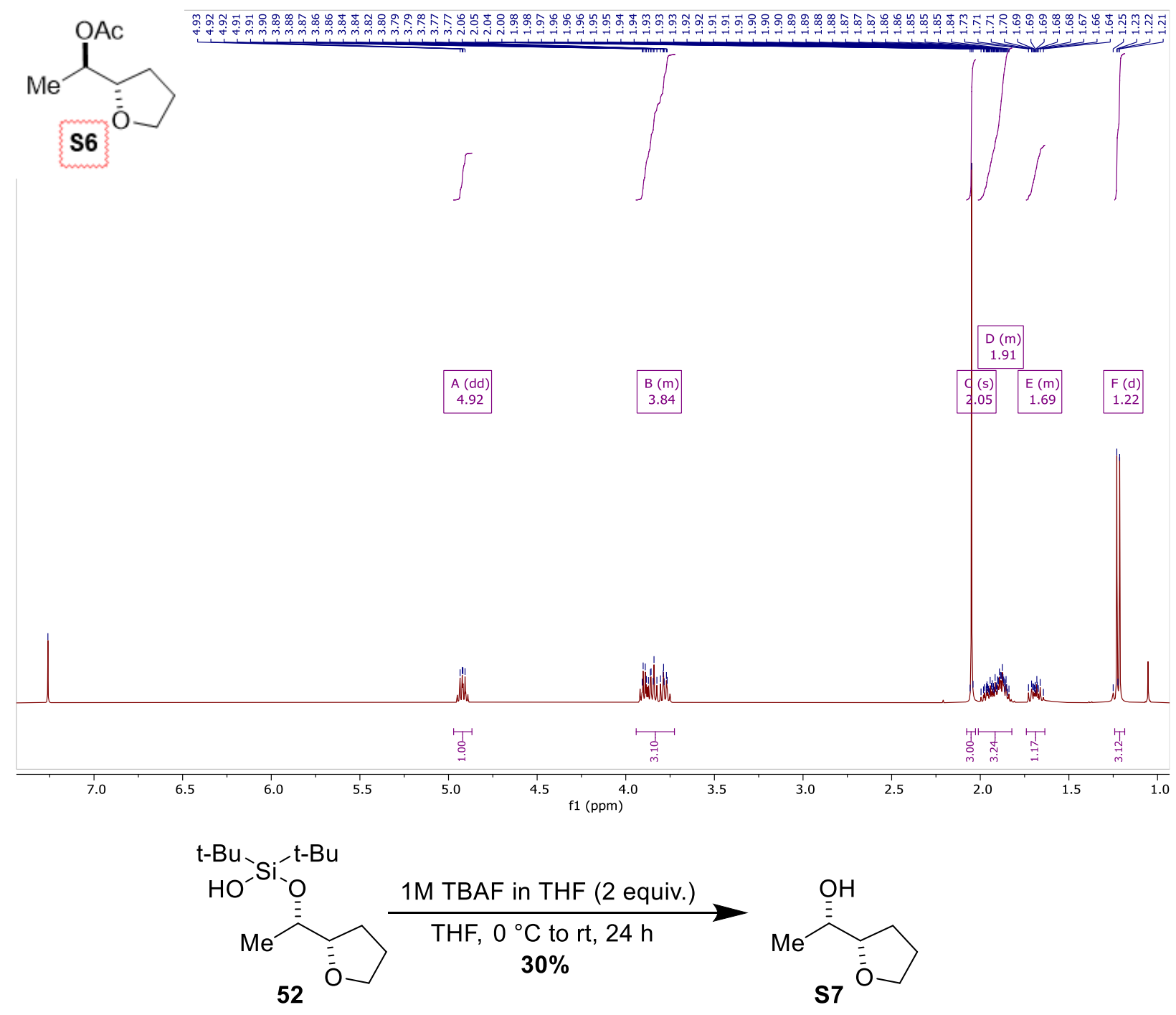

A $25 \mathrm{~mL}$ round-bottom flask was charged with a stir-bar, $\mathbf{5 2}(80 \mathrm{mg}, 0.291 \mathrm{mmol})$, and anhydrous THF ( $2 \mathrm{~mL}$ ). The reaction flask was cooled to $0{ }^{\circ} \mathrm{C}$ using an ice-water bath. Subsequently, TBAF (1M solution in THF, $0.58 \mathrm{~mL}, 0.58 \mathrm{mmol}, 2$ equiv.) was added dropwise. The reaction mixture was allowed to warm to room temperature over a period of $24 \mathrm{~h}$. After this time, the reaction was diluted with EtOAc $(10 \mathrm{~mL})$, transferred to a separatory funnel, and washed once with saturated aqueous $\mathrm{NH}_{4} \mathrm{Cl}$ solution $(10 \mathrm{~mL})$. The organic layer was separated, and the aqueous layer was extracted with two additional portions of EtOAc $(2 \times 10 \mathrm{~mL})$. The combined organic layers were washed with brine solution $(15 \mathrm{~mL})$, dried over anhydrous $\mathrm{Na}_{2} \mathrm{SO}_{4}$, and the solvent was removed in vacuo. The resulting residue was purified through silica gel column chromatography (hexane/ethyl acetate $=30: 70)$ to afford $10 \mathrm{mg}(0.086 \mathrm{mmol}, 30 \%)$ of $\mathbf{S 7}$ as a colorless oil. 
S7 is a known compound and has been previously characterized in J. Org. Chem. 1995, 60, 169-175.

\begin{tabular}{|l|l|}
\hline${ }^{1} \mathrm{H} \mathrm{NMR}\left(400 \mathrm{MHz}, \mathrm{CDCl}_{3}\right)$ & Reported ${ }^{1} \mathrm{H} \mathrm{NMR}\left(400 \mathrm{MHz} \mathrm{CDCl}_{3}\right)$ \\
\hline $3.88-3.75(\mathrm{~m}, 2 \mathrm{H})$ & $3.83(\mathrm{~m}, 2 \mathrm{H})$ \\
\hline $3.72-3.61(\mathrm{~m}, 1 \mathrm{H})$ & $3.7-3.5(\mathrm{~m}, 2 \mathrm{H})$ \\
\hline $3.57(\mathrm{dq}, J=7.2,6.3 \mathrm{~Hz}, 1 \mathrm{H})$ & \\
\hline $1.97-1.86(\mathrm{~m}, 3 \mathrm{H})$ & $1.90(\mathrm{~m}, 3 \mathrm{H})$ \\
\hline $1.62-1.52(\mathrm{~m}, 1 \mathrm{H})$ & $1.55(\mathrm{~m}, 1 \mathrm{H})$ \\
\hline $1.15(\mathrm{~d}, J=6.2 \mathrm{~Hz}, 3 \mathrm{H})$ & $1.15(\mathrm{~d}, J=6.0 \mathrm{~Hz}, 3 \mathrm{H})$ \\
\hline
\end{tabular}

${ }^{1} \mathrm{H} \mathrm{NMR}\left(\mathrm{CDCl}_{3}\right)$

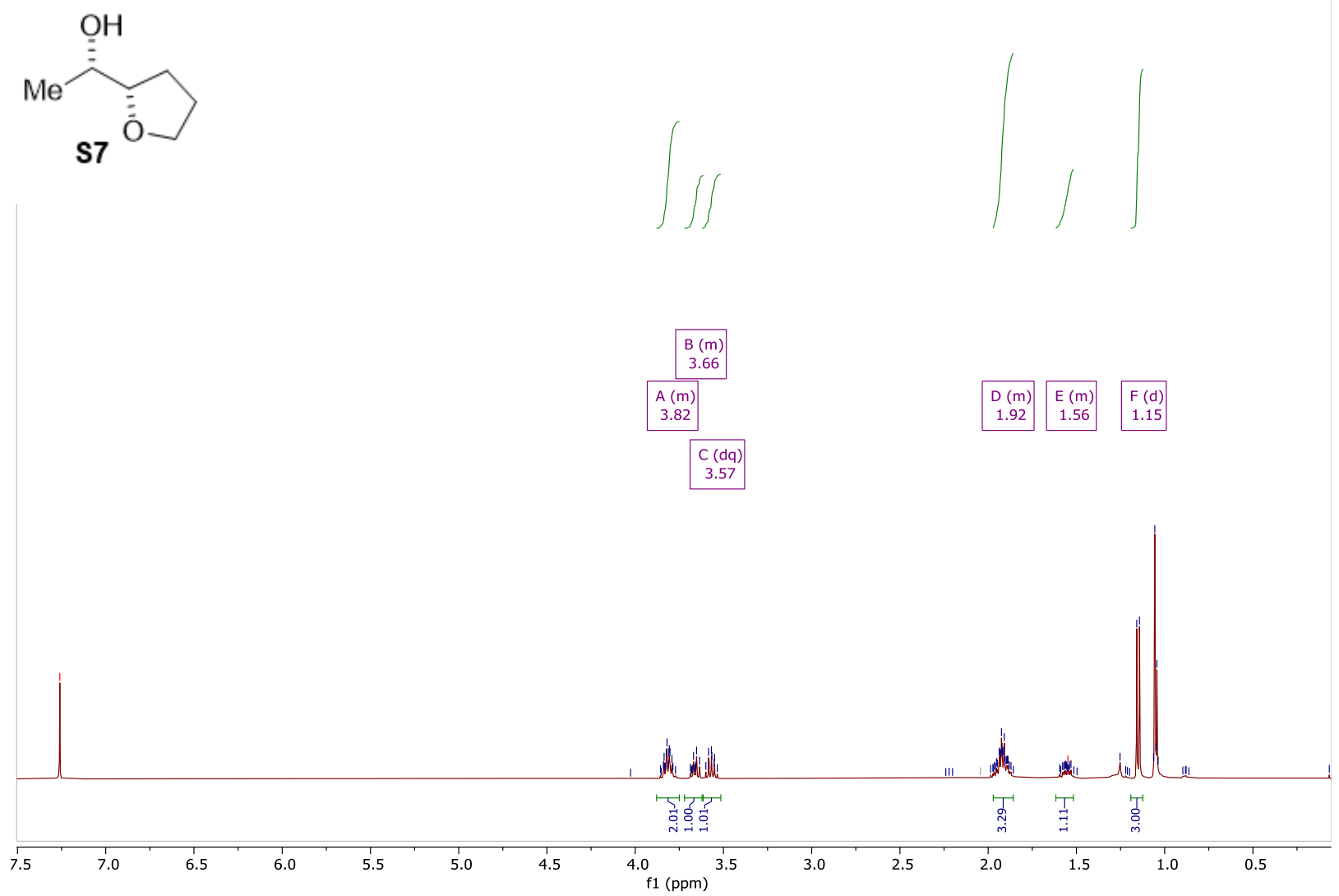


VII. Crystal Structure Data for 41 (CCDC: 2126173)

Crystals suitable for X-ray diffraction grown from DMSO.<smiles>CC(C)(C)[Si]1(C(C)(C)C)COC([C@H](O)CO[Si](C(C)(C)C)(C(C)(C)C)C(C)(C)C)CO1</smiles>

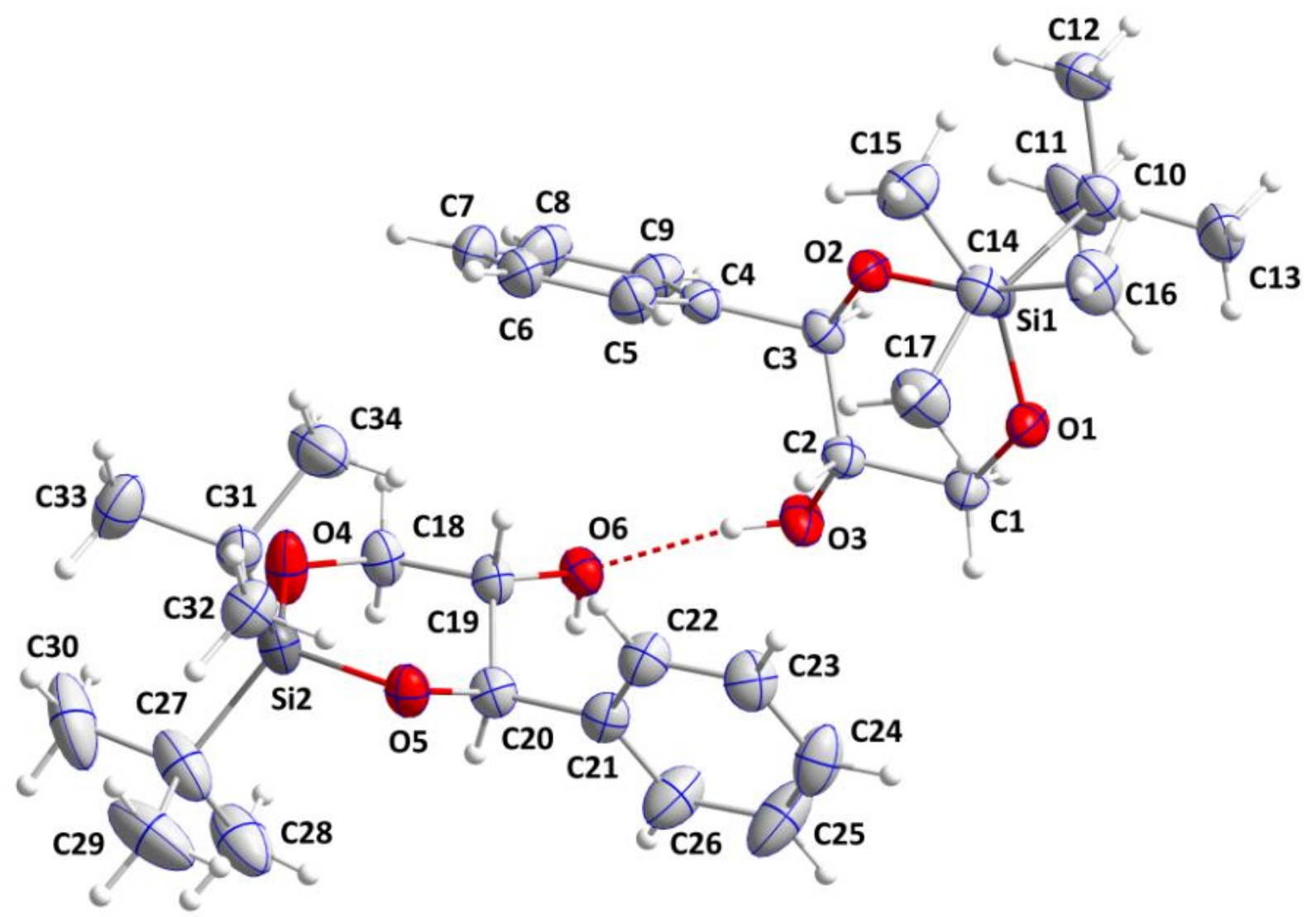

Note: Ellipsoid Contour probability levels are $\mathbf{5 0 \% .}$

\section{Crystal Structure Report for JTM1507}

A colorless, plate-like specimen of $\mathrm{C}_{17} \mathrm{H}_{28} \mathrm{O}_{3} \mathrm{Si}$, approximate dimensions $0.061 \mathrm{~mm} \times 0.207 \mathrm{~mm}$ $x 0.274 \mathrm{~mm}$, was used for the X-ray crystallographic analysis. The X-ray intensity data were measured on a Bruker D8 QUEST PHOTON 3 diffractometer system equipped with a fine-focus sealed tube (MoK $\alpha, \lambda=$ $0.71073 \AA$ ) and a graphite monochromator. 


\section{Table 1: Data collection details for JTM1507.}

$\begin{array}{ccccccccccccc}\text { Axis } & \mathbf{d x} / \mathbf{m m} & \mathbf{2 \theta} /{ }^{\circ} & \boldsymbol{\omega}^{\circ}{ }^{\circ} & \boldsymbol{\varphi}^{\circ}{ }^{\circ} & \chi{ }^{\circ} & \text { Width} /{ }^{\circ} & \text { Frames } & \text { Time/s } & \text { Wavelength/A } & \text { Voltage/kV } & \text { Current/mA } \\ \text { Omega } & 45.068 & 17.45 & -160.37 & 0.00 & 54.80 & 0.50 & 352 & 20.00 & 0.71076 & 50 & 30.0 \\ \text { Omega } & 45.068 & 11.63 & -166.18 & 144.00 & 54.80 & 0.50 & 352 & 20.00 & 0.71076 & 50 & 30.0 \\ \text { Omega } & 45.068 & 11.63 & 9.82 & 72.00 & 54.80 & 0.50 & 352 & 20.00 & 0.71076 & 50 & 30.0 \\ \text { Omega } & 45.068 & 11.63 & -166.18 & -72.00 & 54.80 & 0.50 & 352 & 20.00 & 0.71076 & 50 & 30.0 \\ \text { Phi } & 45.068 & 11.63 & -166.56 & 0.00 & 54.80 & 0.50 & 720 & 20.00 & 0.71076 & 50 & 30.0 \\ \text { Phi } & 45.068 & 11.63 & 9.82 & 0.00 & 54.80 & 0.50 & 720 & 20.00 & 0.71076 & 50 & 30.0 \\ \text { Omega } & 45.068 & 11.63 & -166.18 & -144.00 & 54.80 & 0.50 & 352 & 20.00 & 0.71076 & 50 & 30.0\end{array}$

A total of 3200 frames were collected. The total exposure time was 17.78 hours. The frames were integrated with the Bruker SAINT software package using a Bruker SAINT( Bruker, 2020) algorithm. The integration of the data using a monoclinic unit cell yielded a total of 152776 reflections to a maximum $\theta$ angle of $29.22^{\circ}(0.73 \AA$ resolution $)$, of which 9674 were independent (average redundancy 15.792, completeness $=99.5 \%, \mathrm{R}_{\text {int }}=4.24 \%, \mathrm{R}_{\text {sig }}=2.04 \%$ ) and $7552(78.06 \%)$ were greater than $2 \sigma\left(\mathrm{F}^{2}\right)$. The final cell constants of $\underline{\mathrm{a}}=17.3378(11) \AA, \underline{\mathrm{b}}=9.4226(6) \AA, \underline{\mathrm{c}}=22.1008(15) \AA, \beta=96.770(2)^{\circ}$, volume $=3585.4(4) \AA^{3}$, are based upon the refinement of the XYZ-centroids of 9375 reflections above $20 \sigma(\mathrm{I})$ with $6.000^{\circ}<2 \theta<58.40^{\circ}$. Data were corrected for absorption effects using the Numerical mu Calculated method (SADABS). The ratio of minimum to maximum apparent transmission was 0.957 . The calculated minimum and maximum transmission coefficients (based on crystal size) are 0.9630 and 0.9920 .

The structure was solved and refined using the Bruker SHELXTL Software Package, using the space group $\mathrm{P} 121 / \mathrm{n}$, with $\mathrm{Z}=8$ for the formula unit, $\mathrm{C}_{17} \mathrm{H}_{28} \mathrm{O}_{3} \mathrm{Si}$. The final anisotropic fullmatrix least-squares refinement on $\mathrm{F}^{2}$ with 399 variables converged at $\mathrm{R} 1=4.51 \%$, for the observed data and $w \mathrm{R} 2=12.74 \%$ for all data. The goodness-of-fit was 1.047 . The largest peak in the final difference electron density synthesis was $0.608 \mathrm{e}^{-} / \AA^{3}$ and the largest hole was $-0.412 \mathrm{e}^{-}$ $/ \AA^{3}$ with an RMS deviation of $0.044 \mathrm{e}^{-} / \AA^{3}$. On the basis of the final model, the calculated density 
was $1.143 \mathrm{~g} / \mathrm{cm}^{3}$ and $\mathrm{F}(000), 1344 \mathrm{e}^{-}$.

Table 2. Sample and crystal data for JTM1507.

Identification code
Chemical formula
Formula weight
Temperature
Wavelength
Crystal size
Crystal habit
Crystal system
Space group
Unit cell dimensions

Volume

Z

Density (calculated)

Absorption coefficient

F(000)

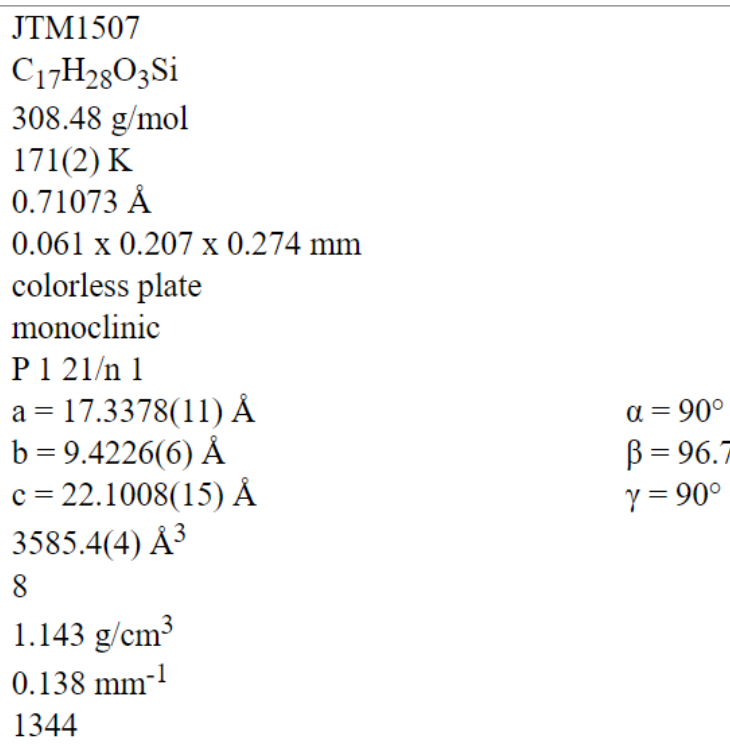

Table 3. Data collection and structure refinement for JTM1507.

\begin{tabular}{|c|c|}
\hline Diffractometer & Bruker D8 QUEST PHOTON 3 diffractometer \\
\hline Radiation source & fine-focus sealed tube $(\mathrm{MoK} \alpha, \lambda=0.71073 \AA)$ \\
\hline Theta range for data collection & 2.35 to $29.22^{\circ}$ \\
\hline Index ranges & $-23<=\mathrm{h}<=23,-12<=\mathrm{k}<=12,-30<=1<=30$ \\
\hline Reflections collected & 152776 \\
\hline Independent reflections & $9674[\mathrm{R}($ int $)=0.0424]$ \\
\hline Coverage of independent reflections & $99.5 \%$ \\
\hline Absorption correction & Numerical mu Calculated \\
\hline Max. and min. transmission & 0.9920 and 0.9630 \\
\hline Structure solution technique & direct methods \\
\hline Structure solution program & SHELXT/5 (Sheldrick, 2015a) \\
\hline Refinement method & Full-matrix least-squares on $\mathrm{F}^{2}$ \\
\hline Refinement program & SHELXL 2018/3 (Sheldrick, 2015b) \\
\hline Function minimized & $\Sigma \mathrm{w}\left(\mathrm{F}_{\mathrm{o}}^{2}-\mathrm{F}_{\mathrm{c}}^{2}\right)^{2}$ \\
\hline Data / restraints / parameters & $9674 / 2 / 399$ \\
\hline Goodness-of-fit on $\mathbf{F}^{2}$ & 1.047 \\
\hline$\Delta / \sigma_{\max }$ & 0.001 \\
\hline Final $\mathbf{R}$ indices & $\mathrm{R} 1=0.0451, \mathrm{wR} 2=0.1161$ \\
\hline & $\mathrm{R} 1=0.0604, \mathrm{wR} 2=0.1274$ \\
\hline Weighting scheme & $\begin{array}{l}\mathrm{w}=1 /\left[\sigma^{2}\left(\mathrm{~F}_{\mathrm{o}}^{2}\right)+(0.0593 \mathrm{P})^{2}+1.3504 \mathrm{P}\right] \\
\text { where } \mathrm{P}=\left(\mathrm{F}_{\mathrm{o}}^{2}+2 \mathrm{~F}_{\mathrm{c}}^{2}\right) / 3\end{array}$ \\
\hline Largest diff. peak and hole & 0.608 and $-0.412 \mathrm{e}^{-3}$ \\
\hline R.M.S. deviation from mean & $0.044 \mathrm{e}^{-3}$ \\
\hline
\end{tabular}




\section{Table 4. Atomic coordinates and equivalent isotropic atomic displacement parameters $\left(\AA^{2}\right)$ for JTM1507.}

$\mathrm{U}(\mathrm{eq})$ is defined as one third of the trace of the orthogonalized $\mathrm{U}_{\mathrm{ij}}$ tensor.

$$
\begin{array}{lllll}
\mathbf{x} / \mathbf{a} & \mathbf{y} / \mathbf{b} & \mathbf{z} / \mathbf{c} & \mathbf{U}(\mathbf{e q})
\end{array}
$$

$\begin{array}{lllll}\text { Si1 } & 0.41202(2) & 0.64845(4) & 0.77070(2) & 0.02711(9)\end{array}$

$\begin{array}{llllll}\mathrm{O} 1 & 0.48704(6) & 0.55816(11) & 0.75214(4) & 0.0332(2)\end{array}$

$\begin{array}{llllll}\mathrm{O} 2 & 0.36045(5) & 0.69349(11) & 0.70611(4) & 0.0306(2)\end{array}$

$\begin{array}{llllll}\mathrm{O} 3 & 0.47541(6) & 0.57216(13) & 0.58694(4) & 0.0380(2)\end{array}$

$\begin{array}{llllll}\text { C1 } & 0.50867(8) & 0.55337(17) & 0.69196(6) & 0.0343(3)\end{array}$

$\begin{array}{llllll}\mathrm{C} 2 & 0.44106(8) & 0.56774(15) & 0.64208(6) & 0.0289(3)\end{array}$

$\begin{array}{llllll}\text { C3 } & 0.39229(8) & 0.70136(14) & 0.64931(6) & 0.0291(3)\end{array}$

$\begin{array}{llllll}\mathrm{C} 4 & 0.32643(8) & 0.71762(15) & 0.59882(6) & 0.0299(3)\end{array}$

$\begin{array}{llllll}\text { C5 } & 0.26006(9) & 0.63509(16) & 0.59722(7) & 0.0363(3)\end{array}$

$\begin{array}{llllll}\text { C6 } & 0.20028(9) & 0.65031(17) & 0.55033(8) & 0.0418(3)\end{array}$

$\begin{array}{lllll}\text { C7 } & 0.20635(10) & 0.7480(2) & 0.50470(7) & 0.0469(4)\end{array}$

$\begin{array}{lllll}\text { C8 } & 0.27147(11) & 0.8320(2) & 0.50616(8) & 0.0488(4)\end{array}$

C9 $0.33154(9) \quad 0.81793(16) 0.55327(7) \quad 0.0383(3)$

$\begin{array}{lllll}\mathrm{C} 10 & 0.44948(9) & 0.81524(16) & 0.81178(7) & 0.0371(3)\end{array}$

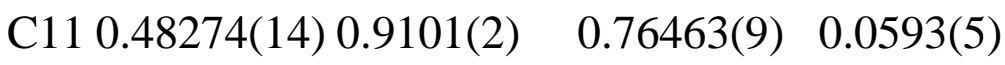

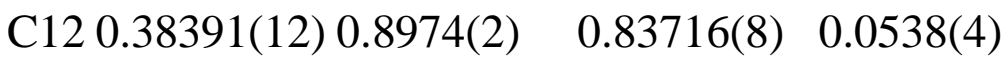

$\begin{array}{llll}\text { C13 0.51342(11) 0.7836(2) } & 0.86392(8) & 0.0520(4)\end{array}$

$\begin{array}{llll}\text { C14 0.34879(8) } & 0.52627(16) & 0.81105(6) & 0.0340(3)\end{array}$

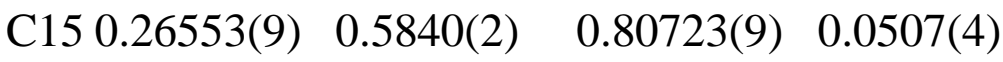

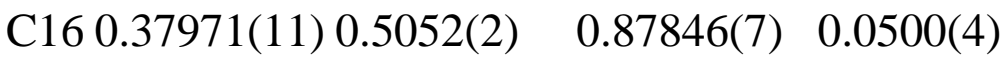

C17 0.34613(11) 0.38153(18) 0.77864(8) $0.0474(4)$

$\begin{array}{lllll}\mathrm{S} i 2 & 0.21281(2) & 0.23474(5) & 0.39083(2) & 0.03549(11)\end{array}$

$\begin{array}{llllll}\mathrm{O} 4 & 0.22707(7) & 0.40187(13) & 0.37400(5) & 0.0499(3)\end{array}$

$\begin{array}{llllll}\text { O5 } & 0.28197(6) & 0.19094(11) & 0.44555(5) & 0.0348(2)\end{array}$

$\begin{array}{lllll}\text { O6 } & 0.40030(6) & 0.51437(11) & 0.47412(4) & 0.0321(2)\end{array}$

C18 0.29389(9) $0.48115(16) 0.39652(6) \quad 0.0347(3)$ 


\begin{tabular}{llcc}
\multicolumn{1}{c}{$\mathbf{x} / \mathbf{a}$} & $\mathbf{y} / \mathbf{b}$ & $\mathbf{z} / \mathbf{c}$ & $\mathbf{U}(\mathbf{e q})$ \\
C19 $0.33330(8)$ & $0.42927(15)$ & $0.45781(6)$ & $0.0312(3)$ \\
C20 $0.35309(8)$ & $0.27067(15)$ & $0.45725(6)$ & $0.0330(3)$ \\
C21 $0.39772(8)$ & $0.22222(15)$ & $0.51658(7)$ & $0.0339(3)$ \\
C22 $0.36350(9)$ & $0.21142(18)$ & $0.57004(7)$ & $0.0401(3)$ \\
C23 $0.40755(10)$ & $0.17391(18)$ & $0.62424(7)$ & $0.0439(4)$ \\
C24 $0.48569(10)$ & $0.1468(2)$ & $0.62549(8)$ & $0.0502(4)$ \\
C25 0.51982(11) $0.1568(3)$ & $0.57270(10)$ & $0.0656(6)$ \\
C26 0.47588(10) & $0.1941(2)$ & $0.51825(8)$ & $0.0518(4)$ \\
C27 0.22131(12) & $0.1283(2)$ & $0.31971(8)$ & $0.0557(5)$ \\
C28 0.30705(13) $0.1353(3)$ & $0.30699(10)$ & $0.0686(6)$ \\
C29 0.19609(17) & $0.9743(3)$ & $0.32499(12)$ & $0.0845(8)$ \\
C30 0.17143(15) & $0.1959(3)$ & $0.26466(9)$ & $0.0864(9)$ \\
C31 0.11802(9) & $0.22417(16)$ & $0.42439(7)$ & $0.0369(3)$ \\
C32 0.10486(10) & $0.07915(18)$ & $0.45214(8)$ & $0.0442(4)$ \\
C33 0.04884(11) & $0.2586(2)$ & $0.37642(10)$ & $0.0581(5)$ \\
C34 0.12050(12) & $0.3362(2)$ & $0.47496(10)$ & $0.0578(5)$
\end{tabular}

\section{Table 5. Bond lengths $(\AA)$ for JTM1507.}

Si1-O1 1.6461(10) Si1-O2 1.6481(10)

Si1-C14 1.8849(14) Si1-C10 1.8913(15)

O1-C1 1.4244(16) O2-C3 1.4315(15)

O3-C2 1.4186(15) O3-H3A 0.841(9)

C1-C2 $1.5175(19) \mathrm{C} 1-\mathrm{H} 1 \mathrm{~A} \quad 0.990000$

C1-H1B $0.990000 \quad$ C2-C3 1.5355(19)

$\mathrm{C} 2-\mathrm{H} 2 \quad 1.000000 \quad \mathrm{C} 3-\mathrm{C} 4 \quad 1.5073(18)$

$\mathrm{C} 3-\mathrm{H} 3 \quad 1.000000 \quad \mathrm{C} 4-\mathrm{C} 5 \quad 1.386(2)$

C4-C9 1.391(2) C5-C6 1.384(2)

C5-H5 $0.950000 \quad \mathrm{C} 6-\mathrm{C} 7 \quad 1.379(3)$

C6-H6 $0.950000 \quad \mathrm{C} 7-\mathrm{C} 8 \quad 1.376(3)$

C7-H7 $0.950000 \quad$ C8-C9 $1.389(2)$

C8-H8 $\quad 0.950000 \quad$ C9-H9 0.950000 

C10-C13 1.531(2) C10-C12 1.535(2)
$\begin{array}{lll}\mathrm{C} 10-\mathrm{C} 11 & 1.536(2) & \mathrm{C} 11- \\ \mathrm{H} 11 \mathrm{~A} & 0.980000\end{array}$
$\begin{array}{llll}\mathrm{C} 11- & 0.980000 & \mathrm{C} 11- & 0.980000 \\ \mathrm{H} 11 \mathrm{~B} & & \end{array}$
$\begin{array}{llll}\mathrm{C} 12- & 0.980000 & \begin{array}{l}\mathrm{C} 12- \\ \mathrm{H} 12 \mathrm{~A}\end{array} & 0.980000\end{array}$
$\begin{array}{llll}\mathrm{C} 12- & 0.980000 & \mathrm{C} 13- & \\ \mathrm{H} 12 \mathrm{C} & & 0.980000\end{array}$
$\begin{array}{llll}\mathrm{C} 13- & 0.980000 & \mathrm{C}^{\mathrm{C}} 13- & 0.980000 \\ \mathrm{H} 13 \mathrm{~B} & & \end{array}$
C14-C16 1.536(2) C14-C15 1.536(2)
$\begin{array}{lll}\mathrm{C} 14-\mathrm{C} 17 & 1.539(2) & \mathrm{C} 15- \\ \mathrm{H} 15 \mathrm{~A} & 0.980000\end{array}$
$\begin{array}{llll}\mathrm{C} 15- & 0.980000 & \mathrm{C} 15- & 0.980000 \\ \mathrm{H} 15 \mathrm{~B} & & \mathrm{H} 15 \mathrm{C} & \end{array}$
$\begin{array}{llll}\text { C16- } & 0.980000 & \text { C16- } & \\ \text { H16A } & & 0.980000\end{array}$
$\begin{array}{llll}\mathrm{C} 16- & 0.980000 & \mathrm{C} 17- & \\ \mathrm{H} 16 \mathrm{C} & & & \\ \mathrm{H} 17 \mathrm{~A} & 0.980000\end{array}$
$\begin{array}{llll}\text { C17- } & 0.980000 & \text { C17- } & 0.980000 \\ \text { H17B } & & \text { H17C } & \end{array}$
$\mathrm{Si} 2-\mathrm{O} 4 \quad 1.6435(12) \mathrm{Si} 2-\mathrm{O} 5 \quad 1.6518(11)$
Si2-C31 1.8837(16) Si2-C27 1.8849(18)
O4-C18 1.4185(17) O5-C20 1.4411(17)
O6-C19 1.4225(16) O6-H6A 0.834(9)
$\begin{array}{lll}\mathrm{C} 18-\mathrm{C} 19 & 1.5240(19) & \mathrm{C} 18- \\ \mathrm{H} 18 \mathrm{~A} & 0.990000\end{array}$
C18-
H18B $0.990000 \quad \mathrm{C} 19-\mathrm{C} 201.534(2)$
C19-H19 1.000000 C20-C21 1.512(2)
C20-H20 1.000000 C21-C26 1.377(2)
C21-C22 1.387(2) C22-C23 1.388(2)
C22-H22 0.950000 C23-C24 1.376(3)
C23-H23 0.950000 C24-C25 1.372(3)
C24-H24 0.950000 C25-C26 1.391(3) 


\begin{tabular}{|c|c|c|c|}
\hline 25 & 50000 & C26-H & 000 \\
\hline 27-C29 & $1.524(3)$ & C27-C30 & $1.545(3)$ \\
\hline C27-C28 & $1.546(3)$ & $\begin{array}{l}\text { C28- } \\
\text { H28A }\end{array}$ & 0.980000 \\
\hline $\begin{array}{l}\text { C28- } \\
\mathrm{H} 28 \mathrm{~B}\end{array}$ & 0.980000 & $\begin{array}{l}\mathrm{C} 28- \\
\mathrm{H} 28 \mathrm{C}\end{array}$ & 0.980000 \\
\hline $\begin{array}{l}\text { C29- } \\
\text { H29A }\end{array}$ & 0.980000 & $\begin{array}{l}\text { C29- } \\
\text { H29B }\end{array}$ & 0.980000 \\
\hline $\begin{array}{l}\mathrm{C} 29- \\
\mathrm{H} 29 \mathrm{C}\end{array}$ & 0.980000 & $\begin{array}{l}\text { C30- } \\
\text { H30A }\end{array}$ & 0.980000 \\
\hline $\begin{array}{l}\text { C30- } \\
\text { H30B }\end{array}$ & 0.980000 & $\begin{array}{l}\text { C30- } \\
\text { H30C }\end{array}$ & 0.980000 \\
\hline C31-C32 & $1.526(2)$ & C31-C34 & $1.535(2)$ \\
\hline $31-\mathrm{C} 33$ & $1.539(2)$ & $\begin{array}{l}\text { C32- } \\
\text { H32A }\end{array}$ & 0.980000 \\
\hline $\begin{array}{l}\text { C32- } \\
\text { H32B }\end{array}$ & 0.980000 & $\begin{array}{l}\text { C32- } \\
\text { H32C }\end{array}$ & 0.980000 \\
\hline $\begin{array}{l}\text { C33- } \\
\text { H33A }\end{array}$ & 0.980000 & $\begin{array}{l}\text { C33- } \\
\text { H33B }\end{array}$ & 0.980000 \\
\hline $\begin{array}{l}\text { C33- } \\
\text { H33C }\end{array}$ & 0.980000 & $\begin{array}{l}\text { C34- } \\
\text { H34A }\end{array}$ & 0.980000 \\
\hline $\begin{array}{l}\text { C34- } \\
\text { H34B }\end{array}$ & 0.980000 & $\begin{array}{l}\text { C34- } \\
\text { H34C }\end{array}$ & 0.980000 \\
\hline
\end{tabular}

\section{Table 6. Bond angles $\left(^{\circ}\right)$ for JTM1507.}

O1-Si1-O2 106.33(5) O1-Si1-C14 108.84(6)

O2-Si1-C14 106.30(6) O1-Si1-C10 108.21(6)

$\begin{array}{lll}\text { O2-Si1-C10 108.81(6) } & \begin{array}{l}\text { C14-Si1- } \\ \text { C10 }\end{array} & \text { 117.80(7) }\end{array}$

C1-O1-Si1 123.48(9) C3-O2-Si1 123.15(8)

C2-O3-H3A 112.1(15) O1-C1-C2 114.19(11)

O1-C1-H1A 108.700000 C2-C1-H1A 108.700000

O1-C1-H1B 108.700000 C2-C1-H1B 108.700000 


\begin{tabular}{|c|c|c|}
\hline H1B & $107.600000 \mathrm{O} 3-$ & $105.08(1$ \\
\hline $2-\mathrm{C} 3$ & 110.94(11) C1-C2-C3 & $112.66(11)$ \\
\hline $3-\mathrm{C} 2-\mathrm{H} 2$ & $109.400000 \mathrm{C} 1-\mathrm{C} 2-\mathrm{H} 2$ & 109.400000 \\
\hline $\mathrm{C} 3-\mathrm{C}$ & 109. & 1) \\
\hline$-\mathrm{C} 2$ & 0) $\mathrm{C} 4$ & \\
\hline $\mathrm{H} 3$ & 108 & 000 \\
\hline $\mathrm{C} 2-\mathrm{C} 3-\mathrm{H} 3$ & 108.900000 C5-C4-C9 & (13) \\
\hline $5-C 4-C 3$ & $121.07(12) \mathrm{C} 9-\mathrm{C}$ & (13) \\
\hline $\mathrm{C} 4$ & 4) $\mathrm{C6}$ & 11 \\
\hline H5 & 119 & \\
\hline H6 & 119 & 000 \\
\hline 8-C7-C6 & 120.01(15) C8-C7-H7 & 0000 \\
\hline $\mathrm{H} 7$ & 120. & 12 \\
\hline C7-C8-H8 & 8-H8 & 11 \\
\hline $\mathrm{C} 4$ & 120.07(15) C8-C9-H9 & 12 \\
\hline H9 & $000 \begin{array}{l}\mathrm{C} 13-\mathrm{C} 10- \\
\mathrm{C} 12\end{array}$ & (13) \\
\hline $\begin{array}{l}\text { C13-C10- } \\
\text { C11 }\end{array}$ & 109.12(15) $\begin{array}{l}\text { C12-C10- } \\
\mathrm{C} 11\end{array}$ & $10^{7}$ \\
\hline $\begin{array}{l}\text { C13-C10- } \\
\text { Si1 }\end{array}$ & 112.13(11) $\begin{array}{l}\text { C12-C10- } \\
\text { Si1 }\end{array}$ & 11 \\
\hline $\begin{array}{l}\text { C11-C10- } \\
\text { Si1 }\end{array}$ & 107.03(10) $\begin{array}{l}\mathrm{C} 10-\mathrm{C} 11- \\
\mathrm{H} 11 \mathrm{~A}\end{array}$ & 00 \\
\hline $\begin{array}{l}\text { C10-C11- } \\
\text { H11B }\end{array}$ & $109.500000 \begin{array}{l}\mathrm{H} 11 \mathrm{~A}-\mathrm{C} 11- \\
\mathrm{H} 11 \mathrm{~B}\end{array}$ & 100 \\
\hline $\begin{array}{l}\text { C10-C11- } \\
\mathrm{H} 11 \mathrm{C}\end{array}$ & $109.500000 \begin{array}{l}\mathrm{H} 11 \mathrm{~A}-\mathrm{C} 11- \\
\mathrm{H} 11 \mathrm{C}\end{array}$ & 109.500000 \\
\hline $\begin{array}{l}\text { H11B-C11- } \\
\text { H11C }\end{array}$ & 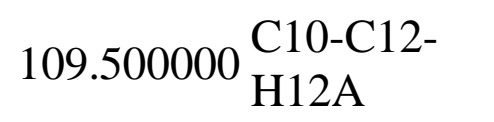 & 109.500000 \\
\hline $\begin{array}{l}\text { C10-C12- } \\
\text { H12B }\end{array}$ & $109.500000 \begin{array}{l}\mathrm{H} 12 \mathrm{~A}-\mathrm{C} 12- \\
\mathrm{H} 12 \mathrm{~B}\end{array}$ & 109.500000 \\
\hline $\begin{array}{l}\mathrm{C} 10-\mathrm{C} 12- \\
\mathrm{H} 12 \mathrm{C}\end{array}$ & $109.500000 \begin{array}{l}\mathrm{H} 12 \mathrm{~A}-\mathrm{C} 12- \\
\mathrm{H} 12 \mathrm{C}\end{array}$ & 109.500000 \\
\hline $\begin{array}{l}\mathrm{H} 12 \mathrm{~B}-\mathrm{C} 12- \\
\mathrm{H} 12 \mathrm{C}\end{array}$ & $109.500000 \begin{array}{l}\mathrm{C} 10-\mathrm{C} 13- \\
\mathrm{H} 13 \mathrm{~A}\end{array}$ & 109.500000 \\
\hline
\end{tabular}




\begin{tabular}{|c|c|c|c|}
\hline 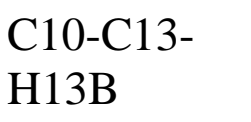 & 109.500000 & 3- & 109 \\
\hline $\begin{array}{l}10-\mathrm{C} 13- \\
13 \mathrm{C}\end{array}$ & 109.500000 & $\begin{array}{l}\text { H13A-C13- } \\
\text { H13C }\end{array}$ & 109 \\
\hline $\begin{array}{l}\text { H13B-C13- } \\
\text { H13C }\end{array}$ & 109.500000 & $\begin{array}{l}\text { C16-C14- } \\
\text { C15 }\end{array}$ & $108.52(13)$ \\
\hline $\begin{array}{l}\text { C16-C14- } \\
\text { C17 }\end{array}$ & $108.96(13)$ & $\begin{array}{l}\text { C15-C14- } \\
\text { C17 }\end{array}$ & 108.18 \\
\hline $\begin{array}{l}\text { 16-C14- } \\
\text { i1 }\end{array}$ & $112.46(11)$ & $\begin{array}{l}\text { C15-C14- } \\
\text { Si1 }\end{array}$ & $110.67(11)$ \\
\hline $\begin{array}{l}17-\mathrm{C} 14- \\
\text { i1 }\end{array}$ & 107. & $\begin{array}{l}\text { C14-C15- } \\
\text { H15A }\end{array}$ & 000 \\
\hline $\begin{array}{l}\text { C14-C15- } \\
\text { H15B }\end{array}$ & 10950 & $\begin{array}{l}\text { H15A-C15- } \\
\text { H15B }\end{array}$ & 00 \\
\hline C15- & 109.500000 & $\begin{array}{l}\mathrm{A}-\mathrm{C} 15- \\
\mathrm{C}\end{array}$ & 000 \\
\hline C15- & 000 & $\begin{array}{l}\text { C14-C16- } \\
\text { H16A }\end{array}$ & 000 \\
\hline & 109.500000 & $\begin{array}{l}\text { A-C16- } \\
B\end{array}$ & 109 \\
\hline 16- & 109 & $\begin{array}{l}\mathrm{H} 16 \mathrm{~A}-\mathrm{C} \\
\mathrm{H} 16 \mathrm{C}\end{array}$ & 109.500000 \\
\hline C16- & 109 & $\begin{array}{l}\text { C14-C17- } \\
\text { H17A }\end{array}$ & 109 \\
\hline $\begin{array}{l}\text { C14-C17- } \\
\text { H17B }\end{array}$ & 109.500000 & $\begin{array}{l}\text { A-C17- } \\
B\end{array}$ & 0000 \\
\hline $\begin{array}{l}\text { C14-C17- } \\
\text { H17C }\end{array}$ & 109.500000 & $\begin{array}{l}\text { H17A-C17- } \\
\text { H17C }\end{array}$ & 109.500000 \\
\hline $\begin{array}{l}\text { H17B-C17- } \\
\text { H17C }\end{array}$ & 109. & O4-Si2-O5 & $106.83(5)$ \\
\hline O4-Si2-C31 & $107.54(7)$ & O5-Si2-C31 & 106 \\
\hline $\mathrm{O} 4-\mathrm{Si} 2-\mathrm{C} 27$ & $107.00(8)$ & O5-Si2-C27 & $110.87(8)$ \\
\hline $\begin{array}{l}\text { C31-Si2- } \\
\text { C27 }\end{array}$ & (7) & $\mathrm{C} 1$ & 12 \\
\hline C20-O5-S & $122.21(9)$ & $\begin{array}{l}\text { C19-O6- } \\
\text { H6A }\end{array}$ & $109.5(15)$ \\
\hline
\end{tabular}




\begin{tabular}{|c|c|c|c|}
\hline C19 & $113.90(11)$ & $\begin{array}{l}\text { O4-C18- } \\
\text { H18A }\end{array}$ & 108.80000 \\
\hline C18 & 88.800000 & $\begin{array}{l}\text { O4-C18- } \\
\mathrm{H} 18 \mathrm{~B}\end{array}$ & 108.8 \\
\hline 18 & 108.800000 & $\begin{array}{l}\text { H18A-C18- } \\
\text { H18B }\end{array}$ & 107. \\
\hline $6-\mathrm{C} 19-$ & 08.15(11) & $\begin{array}{l}\text { O6-C19- } \\
\text { C20 }\end{array}$ & $112.00(11)$ \\
\hline $\begin{array}{l}\text { C18-C19- } \\
\text { C20 }\end{array}$ & $12.50(11)$ & $\begin{array}{l}\text { O6-C19- } \\
\text { H19 }\end{array}$ & 108.000000 \\
\hline & & $\begin{array}{l}\text { C20-C19- } \\
\text { H19 }\end{array}$ & 108.000000 \\
\hline C21 & (11) & $\begin{array}{l}\text { O5-C20- } \\
\text { C19 }\end{array}$ & 108 \\
\hline 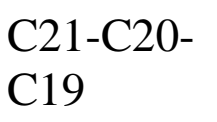 & 12) & $\begin{array}{l}\text { O5-C20- } \\
\mathrm{H} 20\end{array}$ & 000 \\
\hline $20-$ & 000 & $-\mathrm{C} 20-$ & 000 \\
\hline C21- & (14) & $\begin{array}{l}\text { C26-C21- } \\
\text { C20 }\end{array}$ & 4) \\
\hline $\begin{array}{l}22-\mathrm{C} 21- \\
20\end{array}$ & $22.29(13)$ & $\begin{array}{l}\text { C21-C22- } \\
\text { C23 }\end{array}$ & 15) \\
\hline & $11 \mathrm{c}$ & $-\mathrm{C} 22-$ & 119 \\
\hline $\begin{array}{l}\text { C24-C23- } \\
\text { C22 }\end{array}$ & $120.34(15)$ & $\begin{array}{l}\mathrm{C} 24-\mathrm{C} 23- \\
\mathrm{H} 23\end{array}$ & 119.800000 \\
\hline $\begin{array}{l}\text { C22-C23- } \\
\mathrm{H} 23\end{array}$ & 119.800000 & $\begin{array}{l}\mathrm{C} 25-\mathrm{C} 24- \\
\mathrm{C} 23\end{array}$ & $119.50(16)$ \\
\hline $\begin{array}{l}\mathrm{C} 25-\mathrm{C} 24- \\
\mathrm{H} 24\end{array}$ & 120.300000 & $\begin{array}{l}\mathrm{C} 23-\mathrm{C} 24- \\
\mathrm{H} 24\end{array}$ & 120.300000 \\
\hline $\begin{array}{l}\text { C24-C25- } \\
\text { C26 }\end{array}$ & $120.37(16)$ & $\begin{array}{l}\mathrm{C} 24-\mathrm{C} 25- \\
\mathrm{H} 25\end{array}$ & 119.800000 \\
\hline $\begin{array}{l}\text { C26-C25- } \\
\text { H25 }\end{array}$ & 119.800000 & $\begin{array}{l}\text { C21-C26- } \\
\text { C25 }\end{array}$ & $120.58(16)$ \\
\hline $\begin{array}{l}\text { C21-C26- } \\
\text { H26 }\end{array}$ & 119.700000 & $\begin{array}{l}\text { C25-C26- } \\
\mathrm{H} 26\end{array}$ & 119.700000 \\
\hline $\begin{array}{l}\text { C29-C27- } \\
\text { C30 }\end{array}$ & 108.31(19) & $\begin{array}{l}\text { C29-C27- } \\
\text { C28 }\end{array}$ & 110.2 \\
\hline
\end{tabular}




\begin{tabular}{|c|c|c|c|}
\hline $\mathrm{C} 28$ & $107.46(17)$ & $\begin{array}{l}\text { C29-C } \\
\mathrm{Si} 2\end{array}$ & $113.04(1$ \\
\hline 27 & $110.23(17)$ & $\begin{array}{l}\mathrm{C} 28-\mathrm{C} 27- \\
\mathrm{Si} 2\end{array}$ & $107.46(1$ \\
\hline 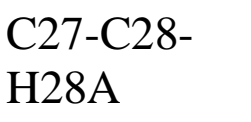 & 0 & $\begin{array}{l}\mathrm{C} 27-\mathrm{C} 28- \\
\mathrm{H} 28 \mathrm{~B}\end{array}$ & 0 \\
\hline & 109 & $\begin{array}{l}\mathrm{C} 27-\mathrm{C} 28- \\
\mathrm{H} 28 \mathrm{C}\end{array}$ & 00 \\
\hline & 109.500000 & $\begin{array}{l}\mathrm{H} 28 \mathrm{~B}-\mathrm{C} 28- \\
\mathrm{H} 28 \mathrm{C}\end{array}$ & 109. \\
\hline & 000 & $\begin{array}{l}\text { C27-C29- } \\
\mathrm{H} 29 \mathrm{~B}\end{array}$ & 109 \\
\hline & & $\begin{array}{l}\mathrm{C} 27 \\
\mathrm{H} 29\end{array}$ & 109. \\
\hline & 000 & $\begin{array}{l}\mathrm{H} 29 \\
\mathrm{H} 29\end{array}$ & 109 \\
\hline & 1 & $\begin{array}{l}\mathrm{C} 27 \\
\mathrm{H} 3 \mathrm{C}\end{array}$ & 00 \\
\hline 30- & & $\begin{array}{l}\mathrm{C} 27 \\
\mathrm{H} 3 \mathrm{C}\end{array}$ & 10 \\
\hline & 000 & $\begin{array}{l}\mathrm{H} 3 \mathrm{C} \\
\mathrm{H} 3 \mathrm{C}\end{array}$ & 1 \\
\hline $1-$ & 14) & $\begin{array}{l}\text { C32-C } \\
\text { C33 }\end{array}$ & t) \\
\hline $1-$ & (15) & $\begin{array}{l}\text { C32- } \\
\text { Si2 }\end{array}$ & 11 \\
\hline - & $107.43(11)$ & $\begin{array}{l}\mathrm{C} 33 \\
\mathrm{Si} 2\end{array}$ & 11 \\
\hline C31- & & $\begin{array}{l}\mathrm{C} 31 \\
\mathrm{H} 32\end{array}$ & 109.500000 \\
\hline C32- & & $\begin{array}{l}\mathrm{C} 31 \\
\mathrm{H} 32\end{array}$ & 109.500000 \\
\hline $\begin{array}{l}\text { H32A-C32- } \\
\text { H32C }\end{array}$ & 109.500000 & $\begin{array}{l}\text { H32B-C32- } \\
\text { H32C }\end{array}$ & 00000 \\
\hline $\begin{array}{l}\text { C31-C33- } \\
\text { H33A }\end{array}$ & 109.500000 & $\begin{array}{l}\text { C31-C33- } \\
\text { H33B }\end{array}$ & 00000 \\
\hline $\begin{array}{l}\text { H33A-C33- } \\
\text { H33B }\end{array}$ & .50 & $\begin{array}{l}\text { C31-C33- } \\
\text { H33C }\end{array}$ & 109.500000 \\
\hline
\end{tabular}



$\begin{array}{lll}\text { H33A-C33- } & 109.500000 & \text { H33B-C33- } \\ \text { H33C } & 109.500000\end{array}$
$\begin{array}{lll}\text { C31-C34- } & 109.500000 & \text { C31-C34- } \\ \text { H34A } & 109.500000\end{array}$
$\begin{array}{lll}\text { H34A-C34- } & 109.500000 & \begin{array}{l}\text { C31-C34- } \\ \text { H34C }\end{array} \\ \text { H34B } & 109.500000\end{array}$
$\begin{array}{lll}\text { H34A-C34- } & 109.500000 & \text { H34B-C34- } \\ \text { H34C } & 109.500000\end{array}$

\section{Table 7. Torsion angles $\left({ }^{\circ}\right)$ for} JTM1507.

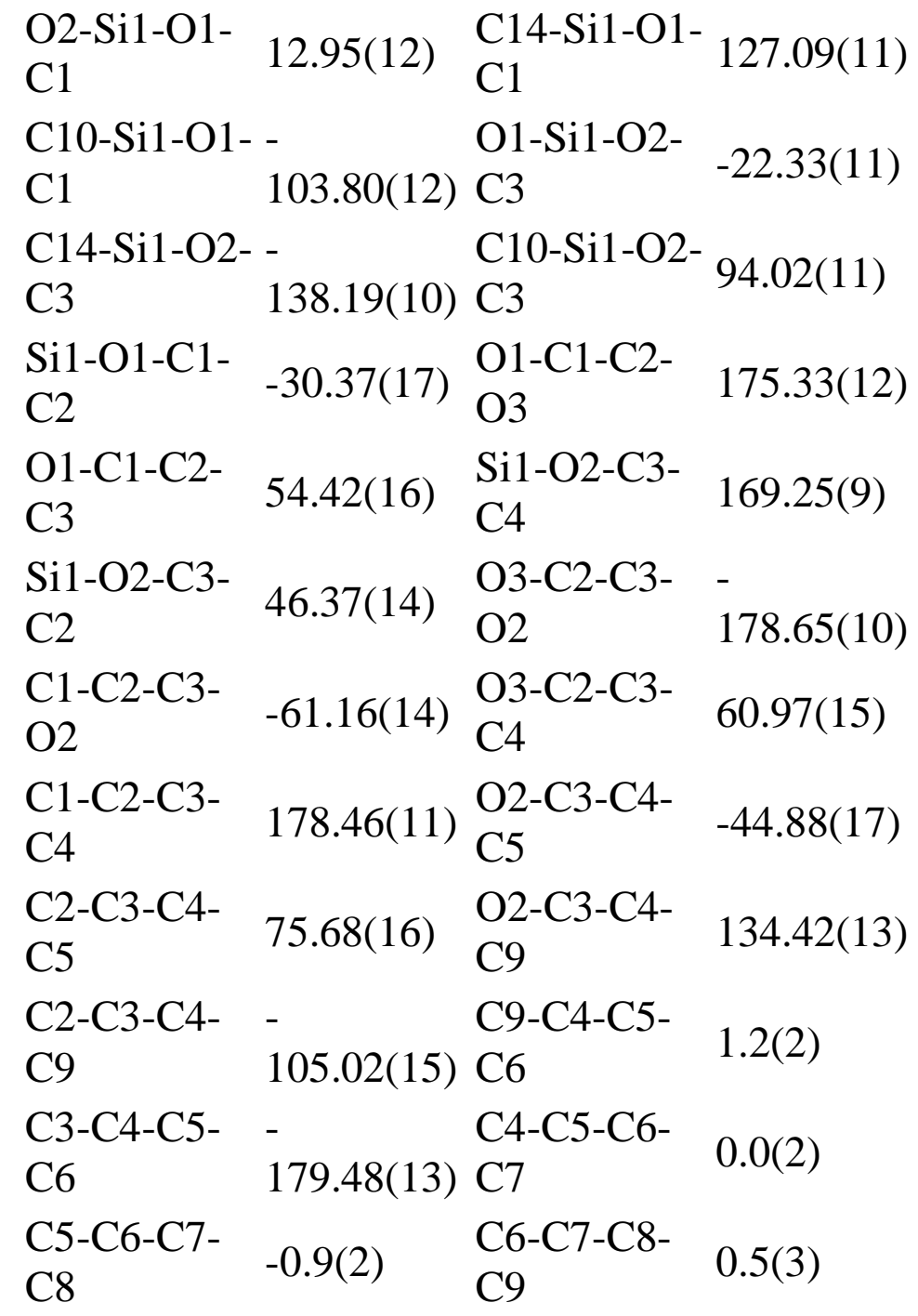




$$
\begin{aligned}
& \begin{array}{llll}
\mathrm{C} 7-\mathrm{C} 8-\mathrm{C} 9- & \text { 0.7(2) } & \mathrm{C} 5-\mathrm{C} 4-\mathrm{C} 9- & -1.6(2) \\
\mathrm{C} 4 & & \mathrm{C} 8 &
\end{array} \\
& \begin{array}{lll}
\text { C3-C4-C9- } & \text { 179.11(14) } & \text { O1-Si1-C10- } \\
\text { C } 8 & & \text {-50.98(13) }
\end{array} \\
& \begin{array}{llll}
\text { O2-Si1-C10- } & \text { C14-Si1- } & \text { 72.91(14) } \\
\text { C13 } & \text { 166.11(11) } & \text { C10-C13 }
\end{array} \\
& \begin{array}{lll}
\text { O1-Si1-C10- } & \text { O2-Si1-C10- } \\
\text { C12 } & \text { 173.50(11) } \mathrm{C} 12
\end{array} \\
& \begin{array}{lll}
\text { C14-Si1- } & \text {-49.61(14) } & \text { O1-Si1-C10- } \\
\text { C11 } & 68.65(14)
\end{array}
\end{aligned}
$$

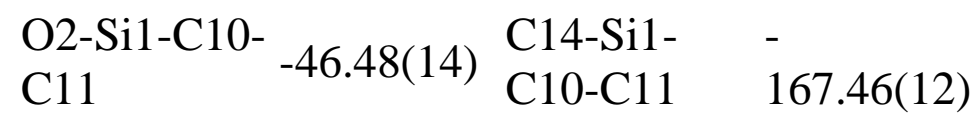

$$
\begin{aligned}
& \begin{array}{lll}
\text { O1-Si1-C14- } & \text { 80.51(12) } & \text { O2-Si1-C14- - } \\
\text { C16 } & \text { C16 } & \text { 165.33(11) }
\end{array} \\
& \begin{array}{llll}
\text { C10-Si1- } & -43.07(14) & \text { O1-Si1-C14- - } \\
\text { C15 } & \text { 157.94(11) }
\end{array} \\
& \begin{array}{lll}
\mathrm{O} 2-\mathrm{Si1}-\mathrm{C} 14- & \text {-43.78(12) } & \begin{array}{l}
\mathrm{C} 10-\mathrm{Si1}- \\
\mathrm{C} 14-\mathrm{C} 15
\end{array}
\end{array} \\
& \begin{array}{ll}
\text { O1-Si1-C14- } & \text {-39.72(12) } \\
\text { C17 } & \text { C17-Si1-C14- }
\end{array} \\
& \begin{array}{lll}
\text { C10-Si1- } & - & \text { O5-Si2-O4- } \\
\text { C14-C17 } & \text { 163.30(11) } \mathrm{C} 18 &
\end{array} \\
& \text { C31-Si2-O4- 123.45(13) C27-Si2-O4- - } \\
& \text { C18 123.45(13) C18 109.77(14) } \\
& \begin{array}{llll}
\mathrm{O} 4-\mathrm{Si} 2-\mathrm{O} 5- & & \mathrm{C} 31-\mathrm{Si} 2-\mathrm{O} 5- \\
\mathrm{C} 20 & -19.86(12) & \mathrm{C} 20 & 134.75(11)
\end{array} \\
& \begin{array}{ll}
\text { C27-Si2-O5- 96.38(12) } & \mathrm{Si2}-\mathrm{O} 4-\mathrm{C} 18- \\
\mathrm{C} 20 & \mathrm{C} 19
\end{array}
\end{aligned}
$$

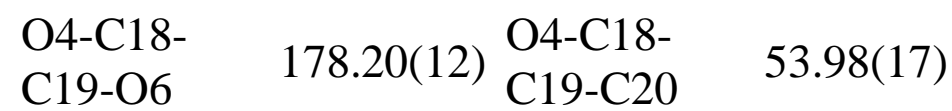

$$
\begin{aligned}
& \begin{array}{ll}
\text { Si2-O5-C20- 169.54(9) } & \begin{array}{l}
\text { Si2-O5-C20- } \\
\text { C219 }
\end{array}
\end{array} \\
& \begin{array}{lll}
\text { O6-C19- } & \text { 175.14(10) } \begin{array}{l}
\text { C18-C19- } \\
\text { C20-O5 }
\end{array} & -62.78(14)
\end{array} \\
& \begin{array}{llll}
\mathrm{O} 6-\mathrm{C} 19- & 53.48(15) & \mathrm{C} 18-\mathrm{C} 19- & \text { 175.56(11) } \\
\mathrm{C} 20-\mathrm{C} 21 & &
\end{array} \\
& \begin{array}{lll}
\mathrm{O} 5-\mathrm{C} 20- & 132.79(15) & \begin{array}{l}
\mathrm{C} 19-\mathrm{C} 20- \\
\mathrm{C} 21-\mathrm{C} 26
\end{array} \\
\mathrm{C} 21-\mathrm{C} 26 & & 106.10(17)
\end{array}
\end{aligned}
$$




\begin{tabular}{|c|c|c|c|}
\hline $\begin{array}{l}\mathrm{O} 5-\mathrm{C} 20- \\
\mathrm{C} 21-\mathrm{C} 22\end{array}$ & $-50.26(19)$ & $\begin{array}{l}\text { C19-C20- } \\
\text { C21-C22 }\end{array}$ & $70.85(18)$ \\
\hline $\begin{array}{l}\text { C26-C21- } \\
\text { C22-C23 }\end{array}$ & $0.5(2)$ & $\begin{array}{l}\text { C20-C21- } \\
\text { C22-C23 }\end{array}$ & $\begin{array}{l}- \\
176.49(15)\end{array}$ \\
\hline $\begin{array}{l}\text { C21-C22- } \\
\text { C23-C24 }\end{array}$ & $-0.1(3)$ & $\begin{array}{l}\text { C22-C23- } \\
\text { C24-C25 }\end{array}$ & $-0.1(3)$ \\
\hline $\begin{array}{l}\mathrm{C} 23-\mathrm{C} 24- \\
\mathrm{C} 25-\mathrm{C} 26\end{array}$ & $0.0(3)$ & $\begin{array}{l}\mathrm{C} 22-\mathrm{C} 21- \\
\mathrm{C} 26-\mathrm{C} 25\end{array}$ & $-0.6(3)$ \\
\hline $\begin{array}{l}\text { C20-C21- } \\
\text { C26-C25 }\end{array}$ & $176.48(18)$ & $\begin{array}{l}\mathrm{C} 24-\mathrm{C} 25- \\
\mathrm{C} 26-\mathrm{C} 21\end{array}$ & $0.3(3)$ \\
\hline $\begin{array}{l}\text { O4-Si2-C27- } \\
\text { C29 }\end{array}$ & $\begin{array}{l}- \\
170.30(17)\end{array}$ & $\begin{array}{l}\text { O5-Si2-C27- } \\
\text { C29 }\end{array}$ & $73.56(18)$ \\
\hline $\begin{array}{l}\text { C31-Si2- } \\
\text { C27-C29 }\end{array}$ & $-49.5(2)$ & $\begin{array}{l}\text { O4-Si2-C27- } \\
\text { C30 }\end{array}$ & $-48.93(15)$ \\
\hline $\begin{array}{l}\text { O5-Si2-C27- } \\
\text { C30 }\end{array}$ & 165.07(13) & $\begin{array}{l}\text { C31-Si2- } \\
\text { C27-C30 }\end{array}$ & $71.85(15)$ \\
\hline $\begin{array}{l}\text { O4-Si2-C27- } \\
\text { C28 }\end{array}$ & $67.89(17)$ & $\begin{array}{l}\text { O5-Si2-C27- } \\
\text { C28 }\end{array}$ & $-48.25(17)$ \\
\hline $\begin{array}{l}\text { C31-Si2- } \\
\text { C27-C28 }\end{array}$ & $\begin{array}{l}- \\
171.32(14)\end{array}$ & $\begin{array}{l}\text { O4-Si2-C31- } \\
\text { C32 }\end{array}$ & $169.97(10)$ \\
\hline $\begin{array}{l}\text { O5-Si2-C31- } \\
\text { C32 }\end{array}$ & $-55.57(12)$ & $\begin{array}{l}\text { C27-Si2- } \\
\text { C31-C32 }\end{array}$ & $69.53(14)$ \\
\hline $\begin{array}{l}\text { O4-Si2-C31- } \\
\text { C34 }\end{array}$ & $-50.65(13)$ & $\begin{array}{l}\text { O5-Si2-C31- } \\
\text { C34 }\end{array}$ & $63.75(13)$ \\
\hline $\begin{array}{l}\text { C27-Si2- } \\
\text { C31-C34 }\end{array}$ & $\begin{array}{l}- \\
171.15(13)\end{array}$ & $\begin{array}{l}\text { O4-Si2-C31- } \\
\text { C33 }\end{array}$ & $67.40(13)$ \\
\hline $\begin{array}{l}\text { O5-Si2-C31- } \\
\text { C33 }\end{array}$ & $\begin{array}{l}- \\
178.19(12)\end{array}$ & $\begin{array}{l}\text { C27-Si2- } \\
\text { C31-C33 }\end{array}$ & $-53.10(15)$ \\
\hline
\end{tabular}

\section{Table 8. Anisotropic atomic displacement parameters $\left(\AA^{2}\right)$ for JTM1507.}

The anisotropic atomic displacement factor exponent takes the form: $-2 \pi^{2}\left[h^{2} a^{* 2} U_{11}+\ldots+2 h k a^{*} b^{*} U_{12}\right]$
$\mathrm{U}_{11}$
$\mathbf{U}_{22}$
$\mathbf{U}_{33}$
$\mathbf{U}_{23}$
$\mathbf{U}_{13}$
$\mathrm{U}_{12}$

Si1 $0.03034(18) 0.02981(18) 0.02140(17)_{0.00020(13)}^{-} 0.00398(13) 0.00062(14)$ 


\section{$\begin{array}{llllll}\mathbf{U}_{11} & \mathbf{U}_{22} & \mathbf{U}_{33} & \mathbf{U}_{23} & \mathbf{U}_{13} & \mathbf{U}_{12}\end{array}$}

$\begin{array}{lllllll}\mathrm{O} 1 & 0.0328(5) & 0.0425(5) & 0.0243(4) & 0.0005(4) & 0.0039(4) & 0.0058(4)\end{array}$

$\begin{array}{lllllll}\mathrm{O} 2 & 0.0336(5) & 0.0366(5) & 0.0221(4) & 0.0005(4) & 0.0057(4) & 0.0053(4)\end{array}$

$\begin{array}{lllllll}\mathrm{O} 3 & 0.0316(5) & 0.0595(7) & 0.0237(5) & -0.0063(4) & 0.0068(4) & 0.0000(5)\end{array}$

$\begin{array}{lllllll}\mathrm{C} 1 & 0.0284(6) & 0.0484(8) & 0.0266(6) & -0.0023(6) & 0.0052(5) & 0.0037(6)\end{array}$

$\begin{array}{lllllll}\mathrm{C} 2 & 0.0295(6) & 0.0343(7) & 0.0237(6) & -0.0022(5) & 0.0060(5) & 0.0001(5)\end{array}$

$\begin{array}{lllllll}\mathrm{C} 3 & 0.0346(7) & 0.0305(6) & 0.0226(6) & 0.0001(5) & 0.0051(5) & -0.0015(5)\end{array}$

$\begin{array}{lllllll}\mathrm{C} 4 & 0.0343(7) & 0.0315(6) & 0.0240(6) & -0.0018(5) & 0.0041(5) & 0.0042(5)\end{array}$

$\begin{array}{lllllll}\text { C5 } & 0.0408(8) & 0.0327(7) & 0.0347(7) & 0.0021(6) & 0.0014(6) & -0.0011(6)\end{array}$

$\begin{array}{lllllll}\mathrm{C} 6 & 0.0379(8) & 0.0391(8) & 0.0462(9) & -0.0101(7) & -0.0041(6) & 0.0019(6)\end{array}$

$\begin{array}{lllllll}\text { C7 } & 0.0479(9) & 0.0549(10) & 0.0351(8) & -0.0052(7) & -0.0075(7) & 0.0181(8)\end{array}$

$\begin{array}{lllllll}\mathrm{C} 8 & 0.0556(10) & 0.0533(10) & 0.0376(8) & 0.0167(7) & 0.0062(7) & 0.0181(8)\end{array}$

$\begin{array}{lllllll}\text { C9 } & 0.0397(8) & 0.0374(7) & 0.0390(8) & 0.0061(6) & 0.0095(6) & 0.0051(6)\end{array}$

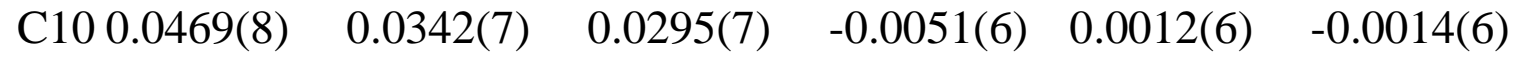

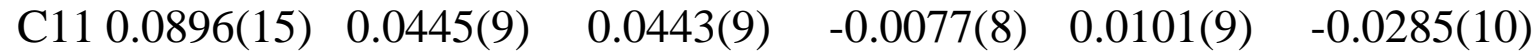

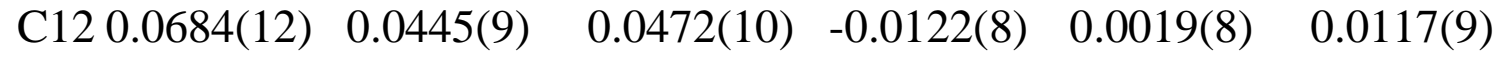

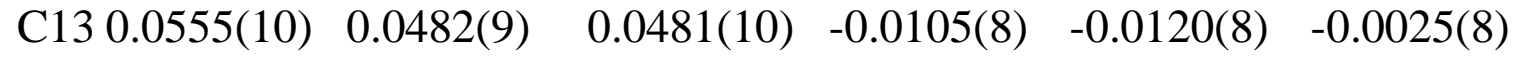

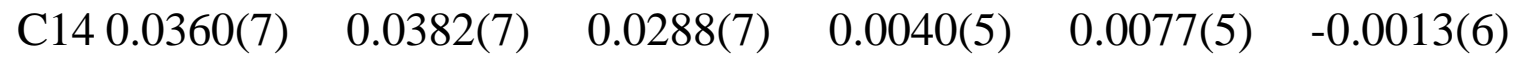

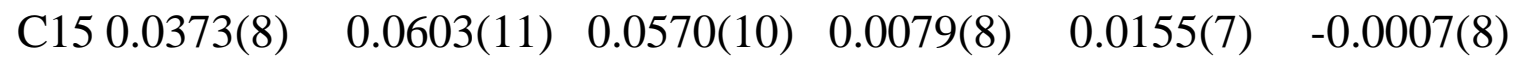

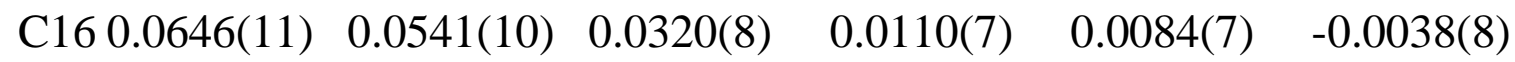

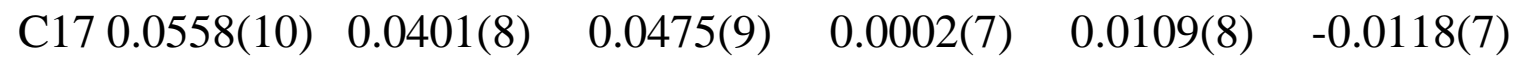

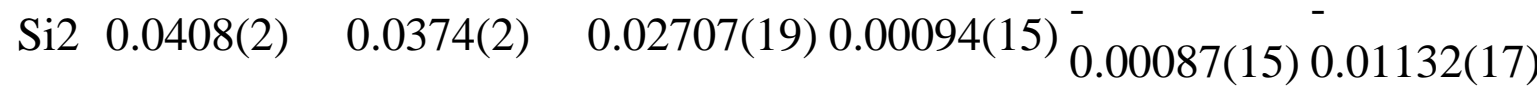

$\begin{array}{lllllll}\mathrm{O} 4 & 0.0561(7) & 0.0461(6) & 0.0419(6) & 0.0142(5) & -0.0175(5) & -0.0204(5)\end{array}$

$\begin{array}{lllllll}\mathrm{O} 5 & 0.0360(5) & 0.0317(5) & 0.0357(5) & 0.0021(4) & 0.0002(4) & -0.0072(4)\end{array}$

$\begin{array}{lllllll}\mathrm{O} 6 & 0.0308(5) & 0.0368(5) & 0.0291(5) & -0.0040(4) & 0.0058(4) & -0.0062(4)\end{array}$

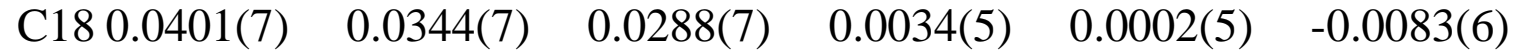

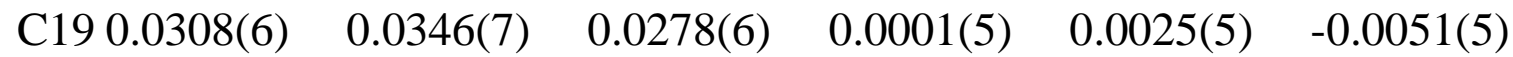

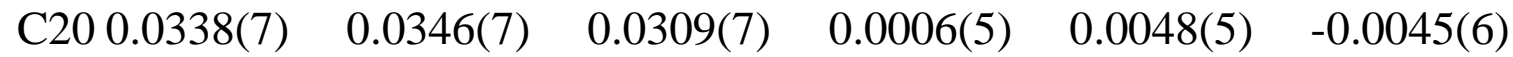

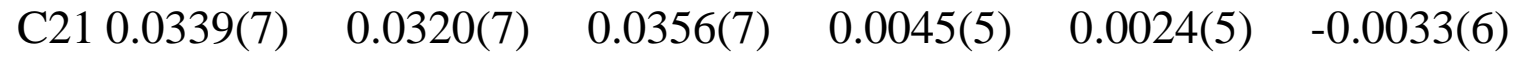

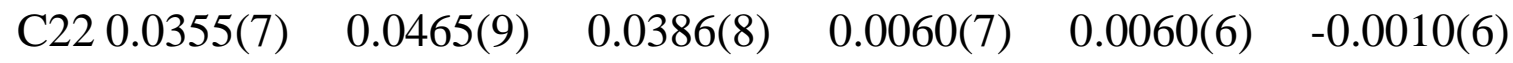

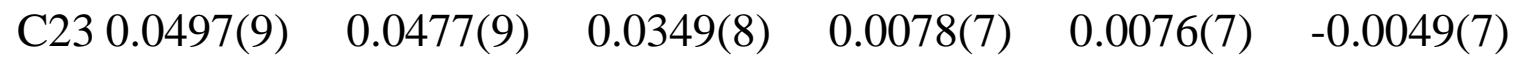

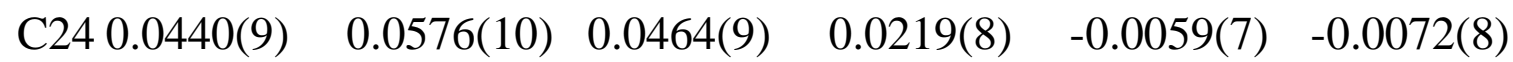

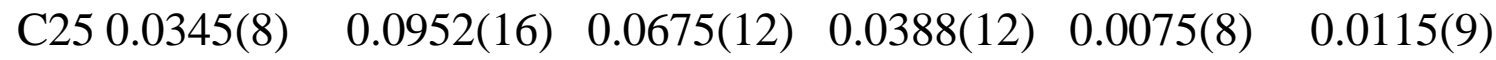

$\begin{array}{lllllll}\mathrm{C} 26 & 0.0414(9) & 0.0681(12) & 0.0478(9) & 0.0227(9) & 0.0131(7) & 0.0099(8)\end{array}$ 
$\begin{array}{llllll}\mathbf{U}_{11} & \mathbf{U}_{22} & \mathbf{U}_{33} & \mathbf{U}_{23} & \mathbf{U}_{13} & \mathbf{U}_{12}\end{array}$

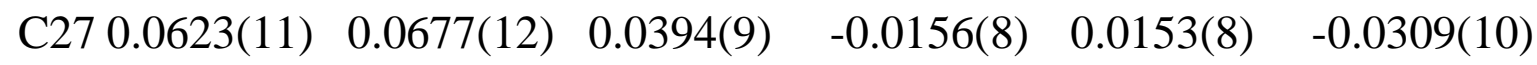

$\begin{array}{lllllll}\mathrm{C} 28 & 0.0654(12) & 0.0924(16) & 0.0513(11) & -0.0274(11) & 0.0210(9) & -0.0228(12)\end{array}$

$\begin{array}{lllllll}\mathrm{C} 29 & 0.113(2) & 0.0683(14) & 0.0799(16) & -0.0378(12) & 0.0459(15) & -0.0372(14)\end{array}$

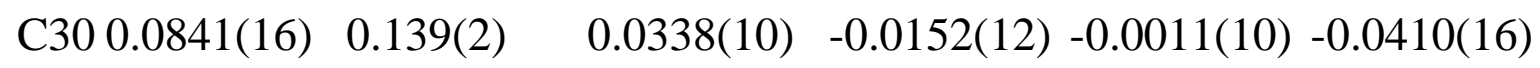

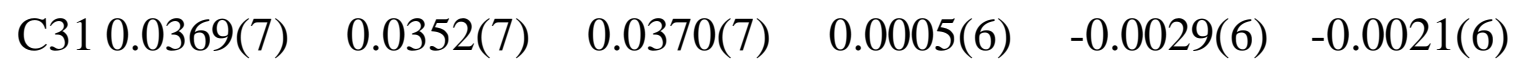

$\begin{array}{lllllll}\mathrm{C} 32 & 0.0426(8) & 0.0461(9) & 0.0443(9) & 0.0090(7) & 0.0071(7) & -0.0015(7)\end{array}$

$\begin{array}{lllllll}\text { C33 0.0482(10) } & 0.0587(11) & 0.0633(12) & 0.0133(9) & -0.0110(9) & 0.0064(8)\end{array}$

$\begin{array}{lllllll}\text { C34 0.0544(11) } & 0.0574(11) & 0.0628(12) & -0.0200(9) & 0.0123(9) & -0.0053(9)\end{array}$

\section{Table 9. Hydrogen atomic}

coordinates and isotropic

atomic displacement

parameters $\left(\AA^{2}\right)$ for

JTM1507.

\section{$\begin{array}{llll}\mathbf{x} / \mathbf{a} & \mathbf{y} / \mathbf{b} & \mathbf{z} / \mathbf{c} & \mathbf{U}(\mathbf{e q})\end{array}$}

H3A $0.4435(10) 0.552(2) 0.5565(7) 0.065(7)$

$\begin{array}{lllll}\text { H1A } & 0.5354 & 0.4623 & 0.6865 & 0.041000 \\ \text { H1B } & 0.5462 & 0.6307 & 0.6874 & 0.041000 \\ \text { H2 } & 0.4071 & 0.4819 & 0.6421 & 0.035000 \\ \text { H3 } & 0.4267 & 0.7866 & 0.6497 & 0.035000 \\ \text { H5 } & 0.2556 & 0.5676 & 0.6285 & 0.044000 \\ \text { H6 } & 0.1550 & 0.5933 & 0.5496 & 0.050000 \\ \text { H7 } & 0.1656 & 0.7574 & 0.4723 & 0.056000 \\ \text { H8 } & 0.2754 & 0.8998 & 0.4749 & 0.059000 \\ \text { H9 } & 0.3761 & 0.8769 & 0.5544 & 0.046000 \\ \text { H11A } 0.5032 & 0.9977 & 0.7844 & 0.089000 \\ \text { H11B } 0.4416 & 0.9335 & 0.7319 & 0.089000 \\ \text { H11C } 0.5246 & 0.8597 & 0.7476 & 0.089000 \\ \text { H12A } 0.4036 & 0.9893 & 0.8529 & 0.081000 \\ \text { H12B } 0.3651 & 0.8427 & 0.8702 & 0.081000 \\ \text { H12C } 0.3412 & 0.9125 & 0.8046 & 0.081000 \\ \text { H13A } 0.5326 & 0.8729 & 0.8828 & 0.078000\end{array}$




\begin{tabular}{lllll} 
& $\mathbf{x} / \mathbf{a}$ & $\mathbf{y} / \mathbf{b}$ & \multicolumn{1}{c}{$\mathbf{z} / \mathbf{c}$} & \multicolumn{1}{c}{$\mathbf{U}(\mathbf{e q})$} \\
H13B & 0.5562 & 0.7336 & 0.8479 & 0.078000 \\
H13C 0.4923 & 0.7241 & 0.8944 & 0.078000 \\
H15A 0.2323 & 0.5144 & 0.8246 & 0.076000 \\
H15B 0.2457 & 0.6014 & 0.7645 & 0.076000 \\
H15C 0.2655 & 0.6730 & 0.8302 & 0.076000 \\
H16A 0.3483 & 0.4334 & 0.8963 & 0.075000 \\
H16B 0.3767 & 0.5951 & 0.9003 & 0.075000 \\
H16C 0.4339 & 0.4736 & 0.8817 & 0.075000 \\
H17A 0.3112 & 0.3177 & 0.7974 & 0.071000 \\
H17B 0.3984 & 0.3405 & 0.7824 & 0.071000 \\
H17C 0.3271 & 0.3942 & 0.7354 & 0.071000 \\
H6A & $0.4326(10)$ & $0.500(2)$ & $0.4497(8)$ & $0.064(6)$ \\
H18A & 0.2788 & 0.5817 & 0.4006 & 0.042000 \\
H18B 0.3318 & 0.4770 & 0.3664 & 0.042000 \\
H19 & 0.2967 & 0.4448 & 0.4889 & 0.037000 \\
H20 & 0.3855 & 0.2524 & 0.4235 & 0.040000 \\
H22 & 0.3096 & 0.2298 & 0.5696 & 0.048000 \\
H23 & 0.3836 & 0.1669 & 0.6606 & 0.053000 \\
H24 & 0.5158 & 0.1214 & 0.6626 & 0.060000 \\
H25 & 0.5738 & 0.1381 & 0.5733 & 0.079000 \\
H26 & 0.5000 & 0.2002 & 0.4819 & 0.062000 \\
H28A 0.3124 & 0.0888 & 0.2681 & 0.103000 \\
H28B 0.3399 & 0.0869 & 0.3398 & 0.103000 \\
H28C 0.3231 & 0.2348 & 0.3051 & 0.103000 \\
H29A 0.2011 & -0.0754 & 0.2867 & 0.127000 \\
H29B 0.1418 & -0.0288 & 0.3333 & 0.127000 \\
H29C 0.2291 & -0.0719 & 0.3583 & 0.127000 \\
H30A 0.1811 & 0.1466 & 0.2273 & 0.130000 \\
H30B 0.1852 & 0.2963 & 0.2616 & 0.130000 \\
H30C 0.1163 & 0.1878 & 0.2702 & 0.130000 \\
H32A 0.0571 & 0.0814 & 0.4719 & 0.066000 \\
H32B 0.1490 & 0.0558 & 0.4824 & 0.066000 \\
H32C 0.1000 & 0.0070 & 0.4200 & 0.066000
\end{tabular}




$\begin{array}{rrrc}\mathbf{x} / \mathbf{a} & \mathbf{y} / \mathbf{b} & \mathbf{z} / \mathbf{c} & \mathbf{U}(\mathbf{e q}) \\ \text { H33A 0.0014 } & 0.2662 & 0.3963 & 0.087000 \\ \text { H33B 0.0427 } & 0.1827 & 0.3459 & 0.087000 \\ \text { H33C 0.0584 } & 0.3487 & 0.3565 & 0.087000 \\ \text { H34A 0.0716 } & 0.3343 & 0.4931 & 0.087000 \\ \text { H34B 0.1278 } & 0.4304 & 0.4577 & 0.087000 \\ \text { H34C 0.1637 } & 0.3156 & 0.5064 & 0.087000\end{array}$

\section{Table 10. Hydrogen bond distances $(\AA)$ and angles $\left(^{\circ}\right)$ for JTM1507.}

\section{Donor- Acceptor- Donor- H H Acceptor Angle}
$\mathrm{O} 3-$
$\mathrm{H} 3 \mathrm{~A} \cdots \mathrm{O} 6$
$0.841(9) 1.918(12)$
$2.7284(14) 161 .(2)$
O6- $\mathrm{H} 6 \mathrm{~A} \cdots \mathrm{O} 3 \# 1$ $0.834(9) 1.990(11) 2.7957(14) 162 .(2)$

Symmetry transformations used to generate equivalent atoms:

$$
\# 1-\mathrm{x}+1,-\mathrm{y}+1,-\mathrm{z}+1
$$

\section{Carbohydrate Synthesis Procedures and Characterization}

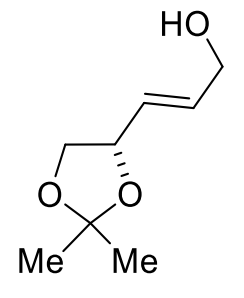

$(+)-53$

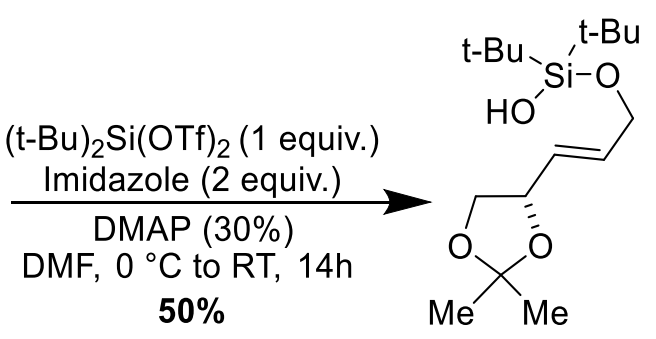

$(+)-54$

A $250 \mathrm{~mL}$ round bottom flask with a magnetic stir bar was charged with imidazole $(0.946 \mathrm{~g}, 13.92 \mathrm{mmol}$, 2 equiv.) and $50 \mathrm{~mL}$ of DMF. After the flask was cooled to $0{ }^{\circ} \mathrm{C}$ using an ice-water bath, di-tert-butylsilyl bis-(trifluoromethanesulfonate) $(2.3 \mathrm{~mL}, 6.96 \mathrm{mmol}, 1$ equiv.) was added dropwise. Following addition, the flask was removed from the ice-water bath, and the clear, colorless solution was stirred for 30 minutes at room temperature. Following this time, the reaction flask was again cooled to $0{ }^{\circ} \mathrm{C}$ and $(+)-53(1.1 \mathrm{~g}$, $6.96 \mathrm{mmol}, 1$ equiv.) was added dropwise followed by $\operatorname{DMAP}(0.254 \mathrm{~g}, 2.08 \mathrm{mmol}, 0.3$ equiv.). The reaction was warmed to room temperature over a period of $14 \mathrm{~h}$. Following this time, the reaction mixture was diluted with ethyl acetate and transferred to a separatory funnel. The solution was washed once with 50 
$\mathrm{mL}$ with $1 \mathrm{M}$ aqueous $\mathrm{NaHCO}_{3}$ solution and then with $400 \mathrm{~mL}$ of cold $\mathrm{H}_{2} \mathrm{O}$. The organic layer was collected, dried with $\mathrm{Na}_{2} \mathrm{SO}_{4}$, and concentrated under reduced pressure. The resulting residue was purified through silica gel column chromatography (DCM/Acetone $=99.8: 0.2)$ to afford $(+)-54(1 \mathrm{~g}, 3.16 \mathrm{mmol}, 50 \%)$ as a colorless oil.

(+)-54 ((S,E)-di-tert-butyl((3-(2,2-dimethyl-1,3-dioxolan-4-yl)allyl)oxy)silanol): [ $\alpha]_{\mathrm{D}}=+22.8$ (c 1.6, $\left.\mathrm{CHCl}_{3}\right)$;; ${ }^{1} \mathrm{H}$ NMR $\left(400 \mathrm{MHz}, \mathrm{CDCl}_{3}\right) \delta 5.89$ (dtd, $\left.J=15.4,4.5,0.8 \mathrm{~Hz}, 1 \mathrm{H}\right), 5.73(\mathrm{ddt}, J=15.3,7.5,1.7 \mathrm{~Hz}, 1 \mathrm{H}), 4.59-$ $4.49(\mathrm{~m}, 1 \mathrm{H}), 4.36$ (ddd, $J=4.6,1.8,0.7 \mathrm{~Hz}, 2 \mathrm{H}), 4.09$ (dd, $J=8.1,6.1 \mathrm{~Hz}, 1 \mathrm{H}), 3.59(\mathrm{t}, J=7.9 \mathrm{~Hz}, 1 \mathrm{H}), 1.43$ (s, 3H), $1.39(\mathrm{~s}, 3 \mathrm{H}), 1.02(\mathrm{~s}, 18 \mathrm{H}) . ;{ }^{13} \mathrm{C}\left\{{ }^{1} \mathrm{H}\right\} \mathrm{NMR}\left(101 \mathrm{MHz}, \mathrm{CDCl}_{3}\right) \delta 133.8,127.2,109.4,77.3,69.6,63.2$, 27.6, 26.8, 26.1, 20.7.; IR: 3430, 2934, 2860, 1473, $651 \mathrm{~cm}^{-1}$; HRMS (ESI) m/z: [M + $\mathrm{H}^{+}$] calculated for $\mathrm{C}_{16} \mathrm{H}_{33} \mathrm{O}_{4} \mathrm{Si} 317.2148$ Found 317.2160.
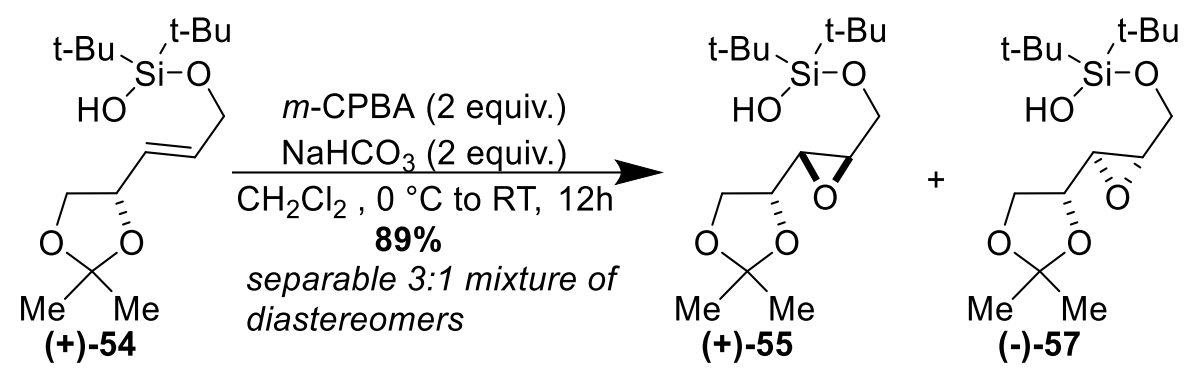

A $50 \mathrm{~mL}$ round-bottom flask was charged with a stir-bar, (+)-54 (0.563 g, 1.78 mmol, 1 equiv.), $\mathrm{NaHCO}_{3}$ ( $0.300 \mathrm{~g}, 3.57 \mathrm{mmol}, 2$ equiv.), and $\mathrm{CH}_{2} \mathrm{Cl}_{2}\left(18 \mathrm{~mL}\right.$ ). The flask was cooled to $0{ }^{\circ} \mathrm{C}$ using an ice-water bath. $m$-CPBA ( $0.613 \mathrm{~g}, 3.55 \mathrm{mmol}, 2$ equiv.) was added in one portion. The heterogenous mixture was allowed to warm to room temperature over a period of 12 hours. Following this time, the mixture was transferred to a separatory funnel with $50 \mathrm{~mL}$ of diethyl ether and washed with one portion of $0.5 \mathrm{M}$ aqueous $\mathrm{NaOH}$ solution. The organic layer was collected, dried with $\mathrm{MgSO}_{4}$, and concentrated under reduced pressure. The resulting residue was purified by chromatography on silica gel (gradient of 0 to $100 \%$ diethyl ether/hexanes) to yield (+)-55 and (-)-57 in a ratio of 3:1 (530 mg, 89\% combined yield).

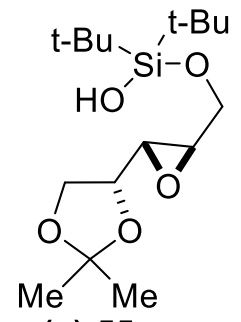

$(+)-55$

di-tert-butyl(((2R,3S)-3-((R)-2,2-dimethyl-1,3-dioxolan-4-yl)oxiran-2-yl)methoxy)silanol

Compound 55 (major diastereomer): $[\alpha]_{\mathrm{D}}=+13.1^{\circ}\left(\mathrm{c}=1, \mathrm{CHCl}_{3}\right) ;{ }^{1} \mathrm{H} \mathrm{NMR}\left(400 \mathrm{MHz}, \mathrm{CDCl}_{3}\right) \delta 4.22-4.09$ $(\mathrm{m}, 2 \mathrm{H}), 3.98-3.90(\mathrm{~m}, 2 \mathrm{H}), 3.85(\mathrm{dd}, J=12.3,5.4 \mathrm{~Hz}, 1 \mathrm{H}), 3.12$ (ddd, $J=5.3,3.0,2.2 \mathrm{~Hz}, 1 \mathrm{H}), 3.09-3.03$ $(\mathrm{m}, 1 \mathrm{H}), 1.48(\mathrm{~s}, 3 \mathrm{H}), 1.39(\mathrm{~s}, 3 \mathrm{H}), 1.05(\mathrm{~s}, 9 \mathrm{H}), 1.04(\mathrm{~s}, 9 \mathrm{H}) .{ }^{13} \mathrm{C} \mathrm{NMR}\left(101 \mathrm{MHz}, \mathrm{CDCl}_{3}\right) \delta$ 109.9, 75.5, 66.9, $63.2,57.7,56.1,27.4,27.3,26.5,25.2,20.6,20.5$. IR 3499, 2974, 1478, 1387, $1010 \mathrm{~cm}^{-1}$.; HRMS (ESI) $\mathrm{m} / \mathrm{z}:\left[\mathrm{M}+\mathrm{Na}^{+}\right]$calculated for $\mathrm{C} 16 \mathrm{H} 32 \mathrm{NaO} 5 \mathrm{Si} 355.1911$ Found 355.1902. 


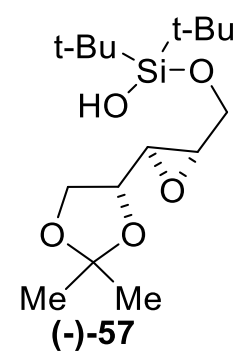

di-tert-butyl(((2S,3R)-3-((R)-2,2-dimethyl-1,3-dioxolan-4-yl)oxiran-2-yl)methoxy)silanol

Compound 57 (minor diastereomer): $[\alpha]_{D}=-5.11^{\circ}\left(\mathrm{c}=1.9, \mathrm{CHCl}_{3}\right) ;{ }^{1} \mathrm{H} \mathrm{NMR}\left(400 \mathrm{MHz}, \mathrm{CDCl}_{3}\right) \delta 4.21-$ $4.06(\mathrm{~m}, 3 \mathrm{H}), 3.92-3.79(\mathrm{~m}, 2 \mathrm{H}), 3.17(\mathrm{dt}, J=5.1,2.5 \mathrm{~Hz}, 1 \mathrm{H}), 3.14-3.03(\mathrm{~m}, 1 \mathrm{H}), 1.45(\mathrm{~s}, 3 \mathrm{H}), 1.39(\mathrm{~s}$, 3H), 1.05 (s, 9H), 1.04 (s, 9H). $\left.{ }^{13} \mathrm{C} \mathrm{NMR} \mathrm{(101} \mathrm{MHz,} \mathrm{CDCl}{ }_{3}\right) \delta$ 110.0, 75.1, 66.0, 62.8, 55.99, 55.97, 27.4, 27.3, 26.3, 25.5, 20.5. IR 3477, 2934, 2860, 1473, 1376, 1147, $1096 \mathrm{~cm}^{-1}$.; HRMS (ESI) m/z: [M + Na $\left.{ }^{+}\right]$ calculated for C16H32NaO5Si 355.1911, Found 355.1919.

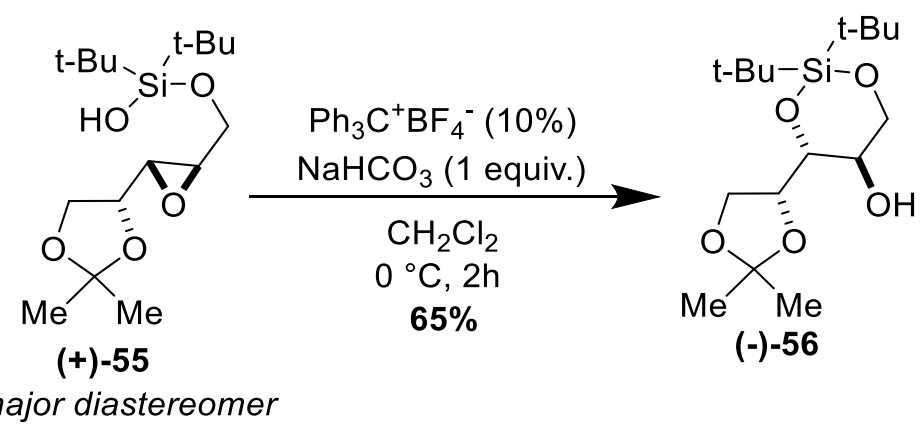

A $5 \mathrm{~mL}$ microwave vial was charged with a stir bar, (+)-55 (66 mg, $0.2 \mathrm{mmol})$ and $\mathrm{NaHCO}_{3}(17 \mathrm{mg}, 0.2$ mmol, 1 equiv.). $2 \mathrm{~mL}$ of dry $\mathrm{CH}_{2} \mathrm{Cl}_{2}$ was added, and the reaction flask was cooled to $0{ }^{\circ} \mathrm{C}$ with an icewater bath. $\mathrm{Ph}_{3} \mathrm{C}^{+} \mathrm{BF}_{4}{ }^{-}(6.6 \mathrm{mg}, 0.02 \mathrm{mmol}, 0.1$ equiv.) was added to the reaction flask. Upon addition, the color of the reaction immediately changed to bright yellow. Following addition, the flask was sealed, and the reaction temperature was maintained at $0{ }^{\circ} \mathrm{C}$ for $2 \mathrm{~h}$. Following this period, the seal was broken, the reaction was diluted with $\mathrm{CH}_{2} \mathrm{Cl}_{2}(2 \mathrm{~mL})$ and quenched with saturated aqueous $\mathrm{NaHCO}_{3}$ solution. The mixture was allowed to stir for 5 minutes during which time the reaction color changed from bright yellow to colorless. The mixture was then transferred to a separatory funnel, and the organic layer was separated. The aqueous layer was extracted with two additional portions of $\mathrm{CH}_{2} \mathrm{Cl}_{2}(2 \times 5 \mathrm{~mL})$. The combined organic layers were washed with brine solution $(5 \mathrm{~mL})$, dried over anhydrous $\mathrm{Na}_{2} \mathrm{SO}_{4}$, and the solvent was removed in vacuo. The resulting residue was purified through silica gel column chromatography $\left(\mathrm{CH}_{2} \mathrm{Cl}_{2} /\right.$ Acetone $\left.=80: 20\right)$ to afford $42 \mathrm{mg}(0.126 \mathrm{mmol}, 65 \%)$ of $(-)-56$ as a colorless oil.

(-)-56 ((4R,5R)-2,2-di-tert-butyl-4-((R)-2,2-dimethyl-1,3-dioxolan-4-yl)-1,3,2-dioxasilinan-5-ol): $[\alpha]_{D}=$ $-20.9^{\circ}$ (c = 1, $\mathrm{CHCl}_{3}$ ).; ${ }^{1} \mathrm{H} \mathrm{NMR}\left(400 \mathrm{MHz}, \mathrm{CDCl}_{3}\right) \delta 4.80$ (ddd, $\left.J=6.4,4.5,1.9 \mathrm{~Hz}, 1 \mathrm{H}\right), 4.70$ (dd, J = 6.4, 2.3 $\mathrm{Hz}, 1 \mathrm{H}), 4.12-4.00(\mathrm{~m}, 2 \mathrm{H}), 3.95(\mathrm{dd}, J=10.3,2.0 \mathrm{~Hz}, 1 \mathrm{H}), 3.88(\mathrm{~d}, J=4.4 \mathrm{~Hz}, 2 \mathrm{H}), 1.51(\mathrm{~s}, 3 \mathrm{H}), 1.34(\mathrm{~s}$, 3H), $1.01(\mathrm{~s}, 9 \mathrm{H}), 1.00(\mathrm{~s}, 9 \mathrm{H}) . ;{ }^{13} \mathrm{C}\left\{{ }^{1} \mathrm{H}\right\} \mathrm{NMR}\left(101 \mathrm{MHz}, \mathrm{CDCl}_{3}\right) \delta 113.1,85.5,82.0,81.3,73.6,64.0,27.6$, 27.5, 26.9, 25.2, 20.7, 20.6.; IR: 3420, 2934, 2860, 1130, 1090, $656 \mathrm{~cm}^{-1}$;; HRMS (ESI) m/z: [M + $\left.\mathrm{Na}^{+}\right]$ calculated for $\mathrm{C}_{16} \mathrm{H}_{32} \mathrm{O}_{5} \mathrm{SiNa} 355.1917$ Found 355.1927. 


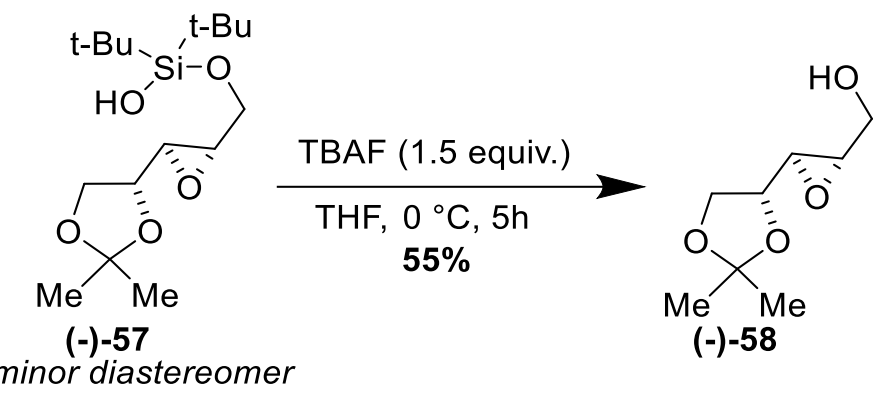

A $10 \mathrm{~mL}$ round-bottom flask was charged with a stir-bar, (-)-57 (74 mg, $0.222 \mathrm{mmol}, 1$ equiv.), and anhydrous THF ( $2 \mathrm{~mL}$ ). The flask was cooled to $0{ }^{\circ} \mathrm{C}$ using a cryogenic cooler. TBAF ( $1 \mathrm{M}$ in THF, $0.350 \mathrm{~mL}$, 1.5 equiv.) was added dropwise. The homogenous mixture was stirred at $0{ }^{\circ} \mathrm{C}$ for 5 hours. Following this time, the reaction mixture was diluted with EtOAc, transferred to a separatory funnel, and washed with one portion of saturated aqueous $\mathrm{NH}_{4} \mathrm{Cl}$ solution. The organic layer was collected, dried with $\mathrm{MgSO}_{4}$, and concentrated under reduced pressure. The resulting residue was purified by chromatography on silica gel (gradient of 0 to $100 \%$ EtOAc/hexanes) to yield (-)-58 (colorless oil, $21 \mathrm{mg}, 0.121 \mathrm{mmol}, 55 \%$ yield).

58 is a known compound. We have compared our measurements with those reported in Org. Lett. 2019, $21,7741-7745$. Note that the NMR solvent was $\mathrm{CDCl}_{3}$ and shifts are in ppm.

\begin{tabular}{|l|l|l|l|}
\hline${ }^{1} \mathrm{H}$ NMR Shifts & Reported ${ }^{1} \mathrm{H}$ NMR Shifts & $\begin{array}{l}{ }^{13} \mathrm{C} \text { NMR } \\
\text { Shifts }\end{array}$ & Reported ${ }^{13} \mathrm{C}$ NMR Shifts \\
\hline $4.15-4.02(\mathrm{~m}, 2 \mathrm{H})$ & $4.15-4.08(\mathrm{~m}, 2 \mathrm{H})$ & 110.2 & 110.2 \\
\hline $3.98-3.89(\mathrm{~m}, 1 \mathrm{H})$ & $\begin{array}{l}3.96(\mathrm{ddd}, \mathrm{J}=12.7,5.0,2.3 \mathrm{~Hz}, \\
1 \mathrm{H})\end{array}$ & 75.3 & 75.3 \\
\hline $3.89-3.80(\mathrm{~m}, 1 \mathrm{H})$ & $3.90-3.81(\mathrm{~m}, 1 \mathrm{H})$ & 66.1 & 66.2 \\
\hline $3.67(\mathrm{dd}, J=12.8,3.9 \mathrm{~Hz}, 1 \mathrm{H})$ & $\begin{array}{l}3.68(\mathrm{ddd}, \mathrm{J}=12.7,7.7,3.7 \mathrm{~Hz}, \\
1 \mathrm{H})\end{array}$ & 60.9 & 60.9 \\
\hline $3.15(\mathrm{dt}, \mathrm{J}=3.9,2.3 \mathrm{~Hz}, 1 \mathrm{H})$ & $3.15(\mathrm{dt}, \mathrm{J}=3.7,2.3 \mathrm{~Hz}, 1 \mathrm{H})$ & 55.5 & 55.5 \\
\hline $3.10(\mathrm{dt}, \mathrm{J}=6.1,3.0 \mathrm{~Hz}, 1 \mathrm{H})$ & $3.11(\mathrm{dd}, \mathrm{J}=4.8,2.3 \mathrm{~Hz}, 1 \mathrm{H})$ & 55.1 & 55.1 \\
\hline $1.42(\mathrm{~s}, 3 \mathrm{H})$ & $1.43(\mathrm{~s}, 3 \mathrm{H})$ & 26.5 & 26.5 \\
\hline $1.36(\mathrm{~s}, 3 \mathrm{H})$ & $1.36(\mathrm{~s}, 3 \mathrm{H})$ & 25.7 & 25.7 \\
\hline
\end{tabular}

IX. ${ }^{1} \mathrm{H}$ and ${ }^{13} \mathrm{C}$ NMR Spectra for all new compounds (All spectra are recorded in $\mathrm{CDCl}_{3}$ ). 


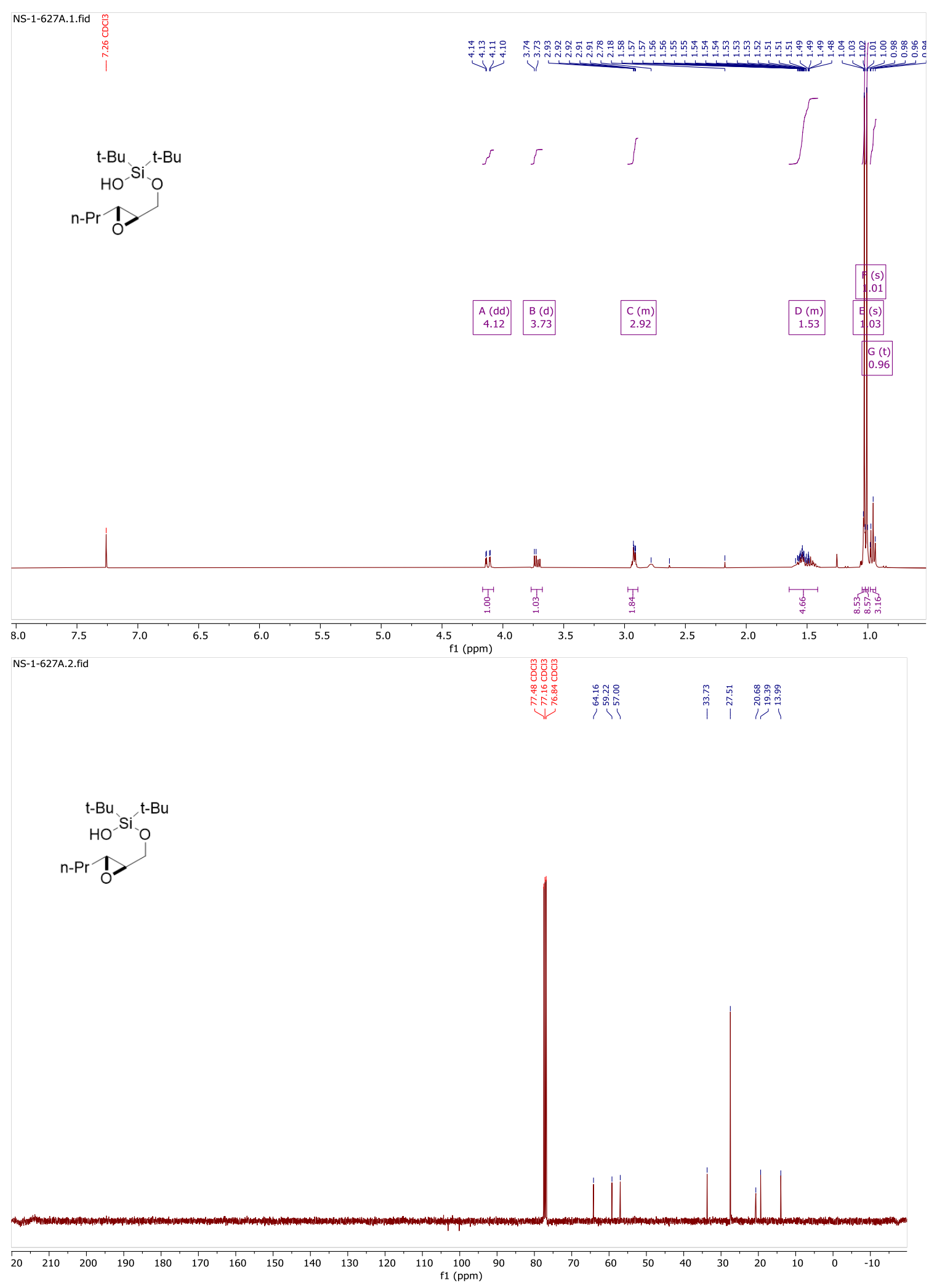

Compound 1 ( ${ }^{1} \mathrm{H}$ NMR: $400 \mathrm{MHz},{ }^{13} \mathrm{C}\left\{{ }^{1} \mathrm{H}\right\}$ NMR: $100 \mathrm{MHz}$ ) 
Compound $2\left({ }^{1} \mathrm{H} N M R: 400 \mathrm{MHz},{ }^{13} \mathrm{C}\left\{{ }^{1} \mathrm{H}\right\}\right.$ NMR: $100 \mathrm{MHz}$ )
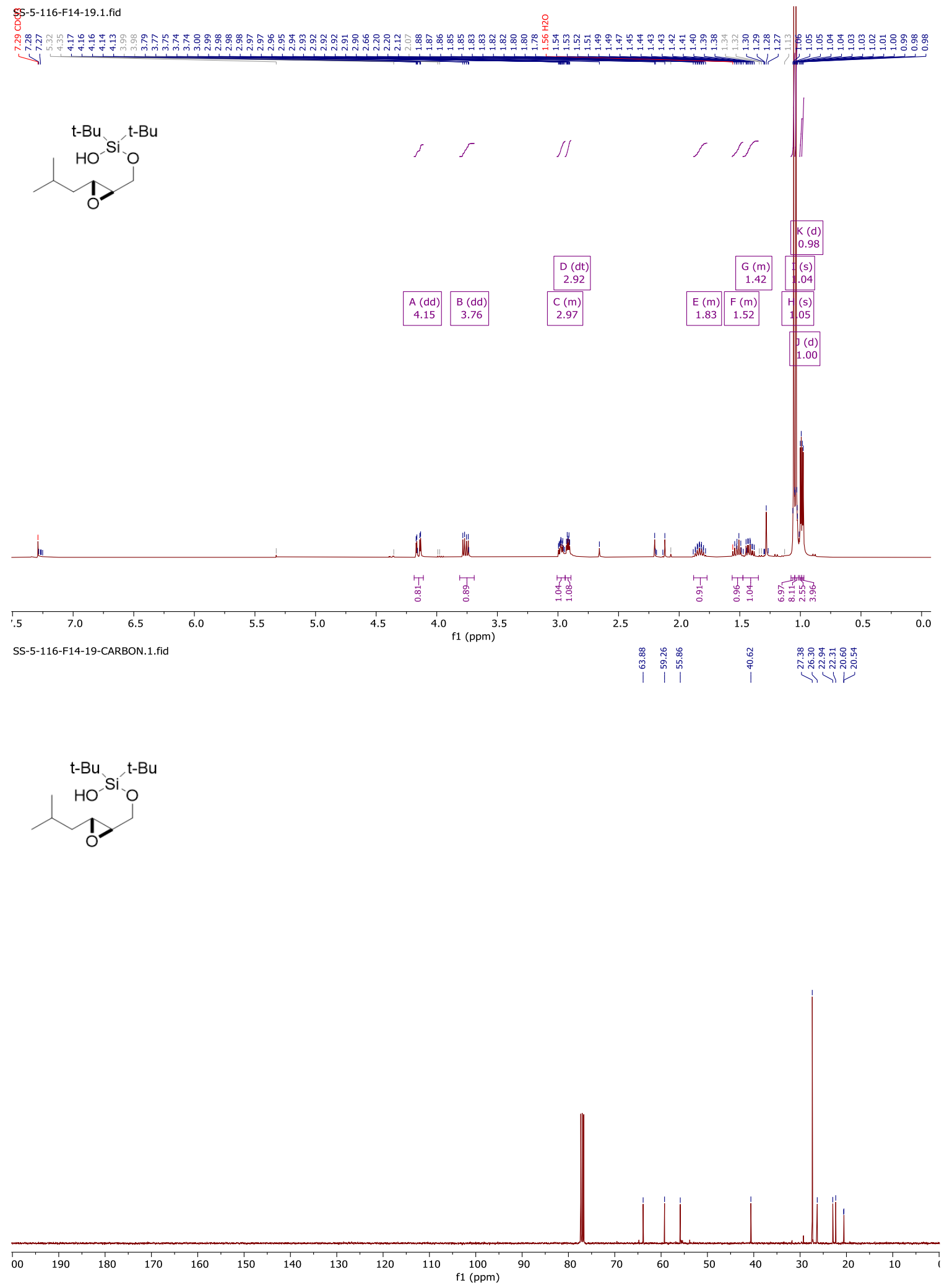
Compound 3 ( ${ }^{1} \mathrm{H}$ NMR: $400 \mathrm{MHz},{ }^{13} \mathrm{C}\left\{{ }^{1} \mathrm{H}\right\}$ NMR: $100 \mathrm{MHz}$ )
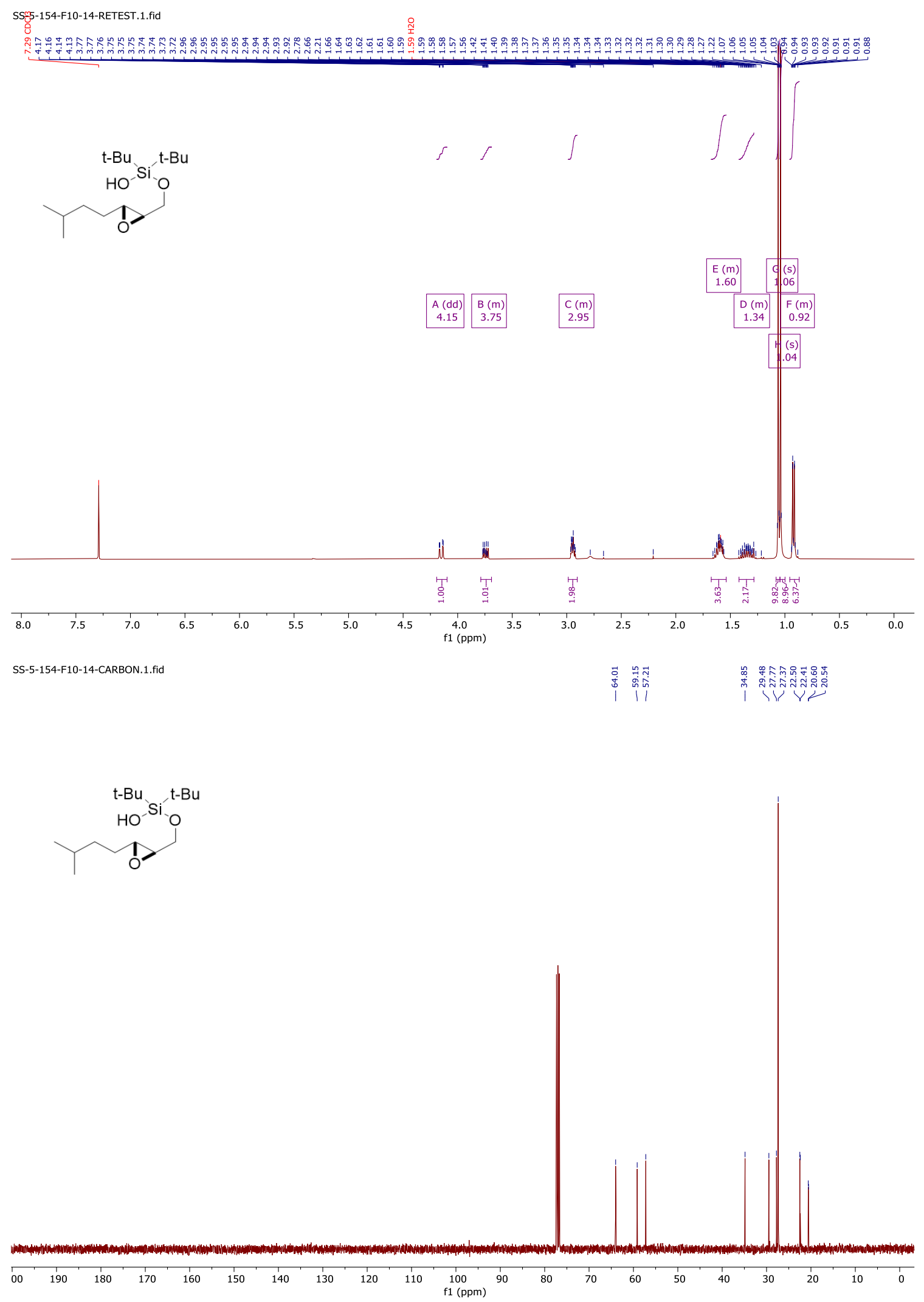
Compound 4 ( ${ }^{1} \mathrm{H}$ NMR: $400 \mathrm{MHz},{ }^{13} \mathrm{C}\left\{{ }^{1} \mathrm{H}\right\}$ NMR: $100 \mathrm{MHz}$ )

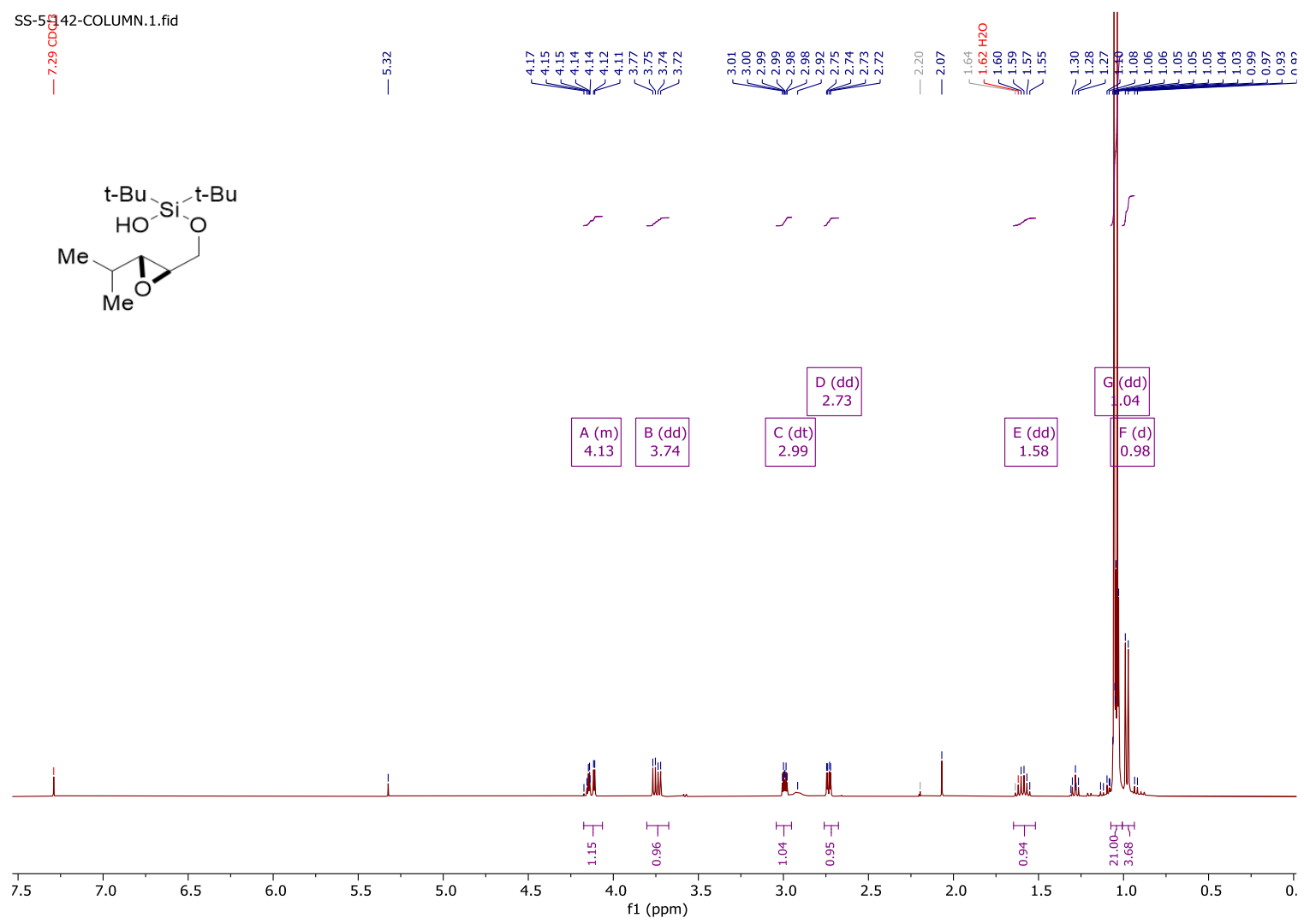

SS-5-142-COLUMN-CARBON.1.fid
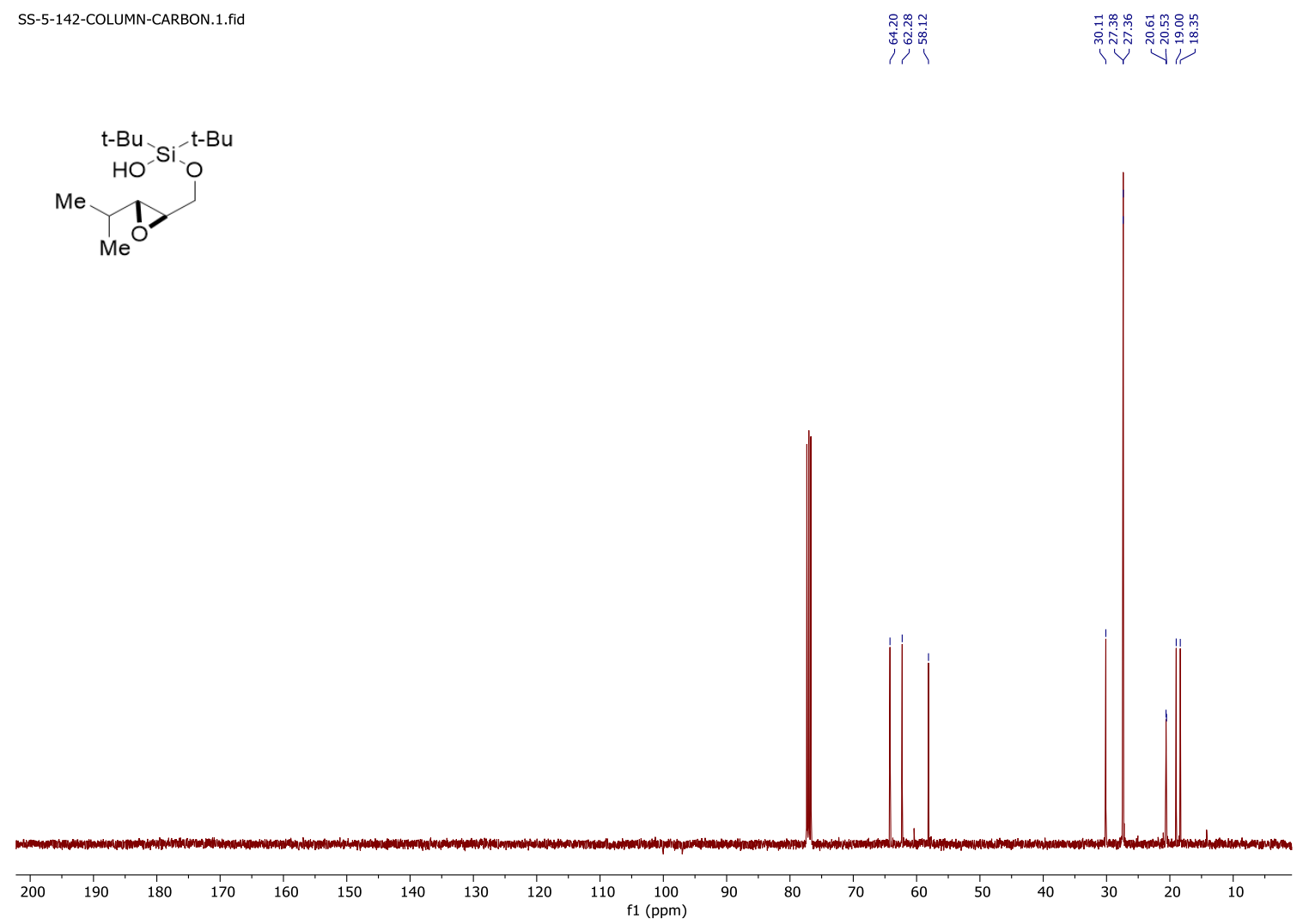
Compound 5 ( ${ }^{1} \mathrm{H}$ NMR: $400 \mathrm{MHz},{ }^{13} \mathrm{C}\left\{{ }^{1} \mathrm{H}\right\}$ NMR: $100 \mathrm{MHz}$ )

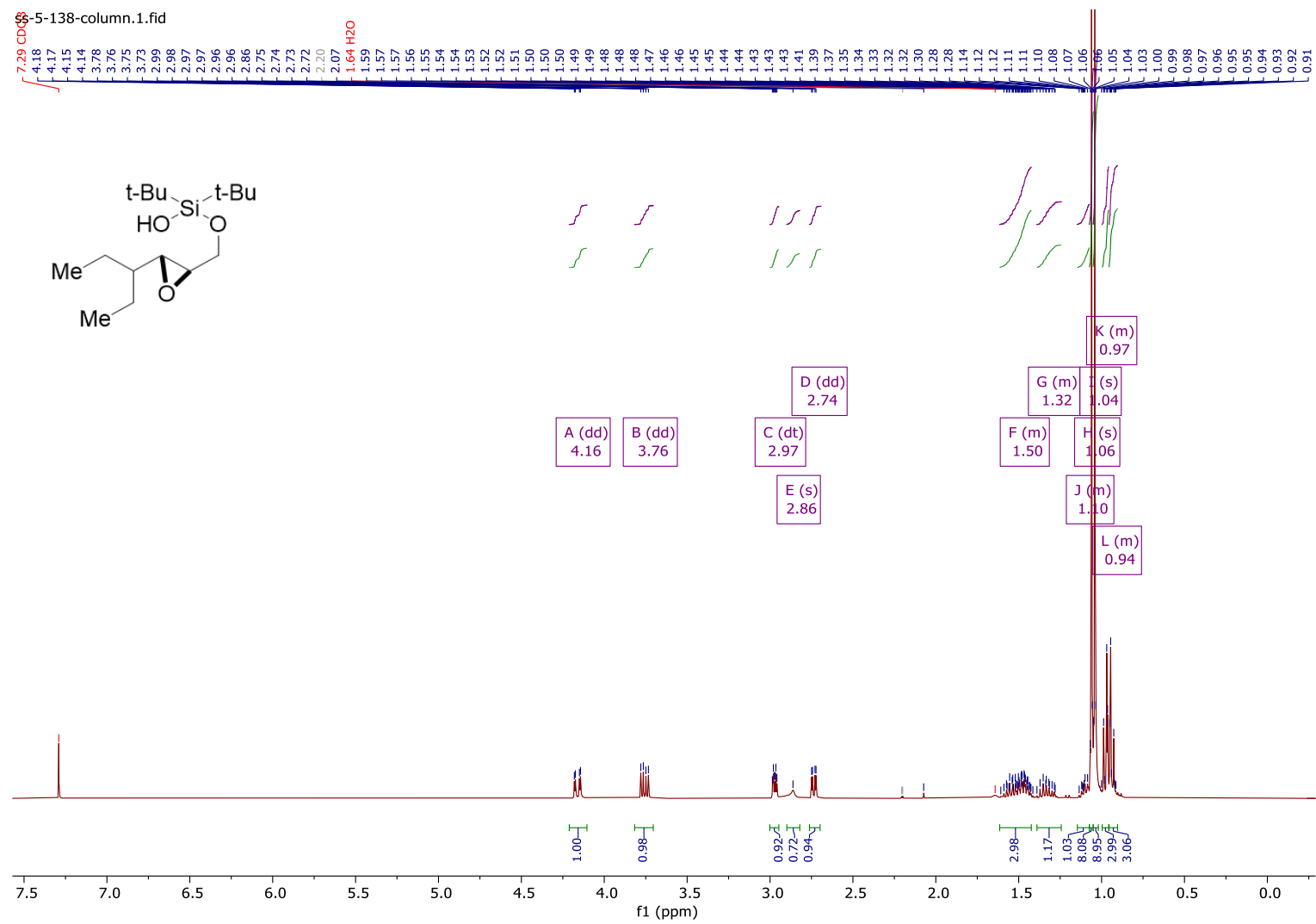

ss-5-138-column-carbon.1.fid

।

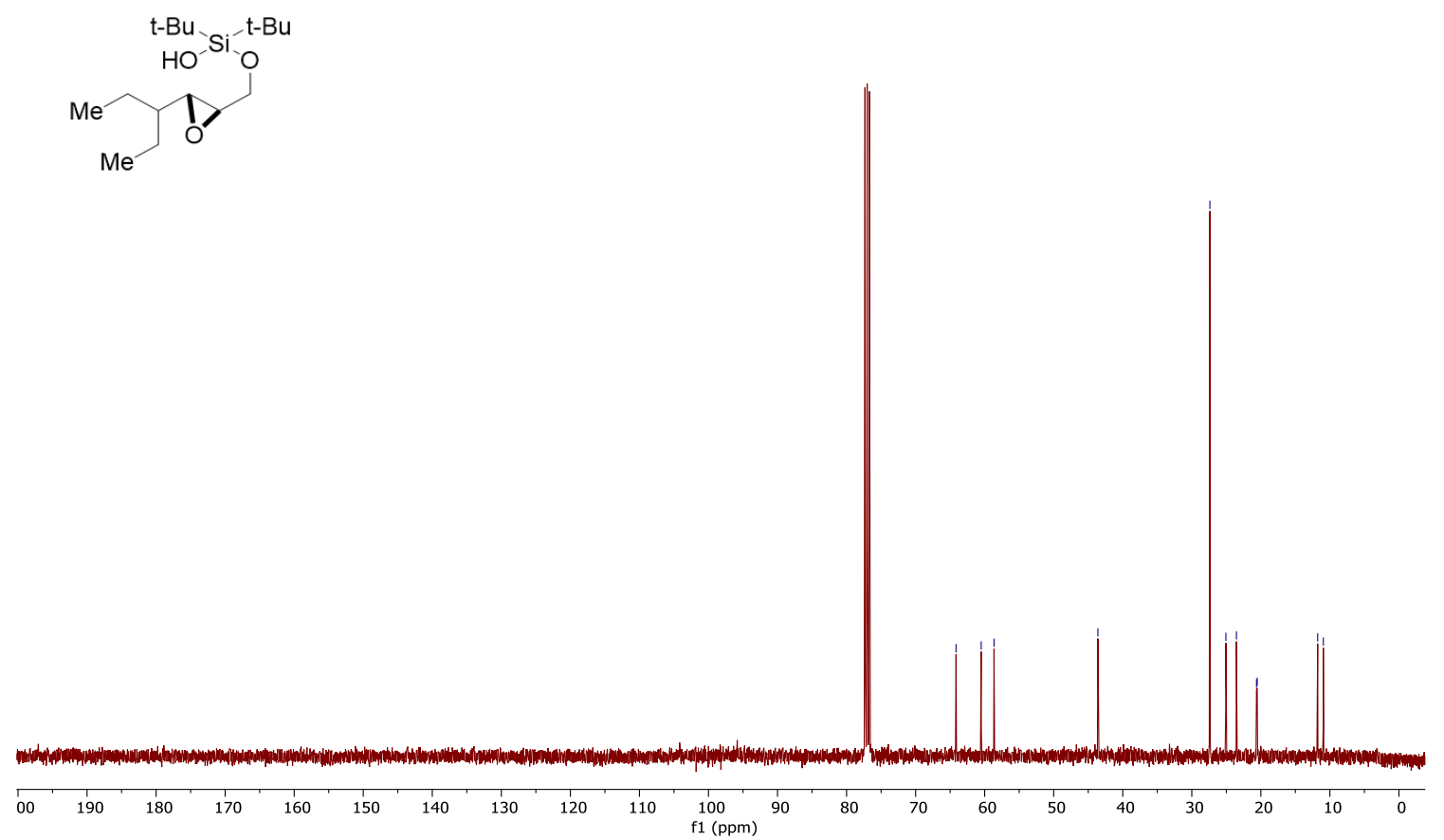


Compound $6\left({ }^{1} \mathrm{H}\right.$ NMR: $400 \mathrm{MHz},{ }^{13} \mathrm{C}\left\{{ }^{1} \mathrm{H}\right\}$ NMR: $100 \mathrm{MHz}$ )
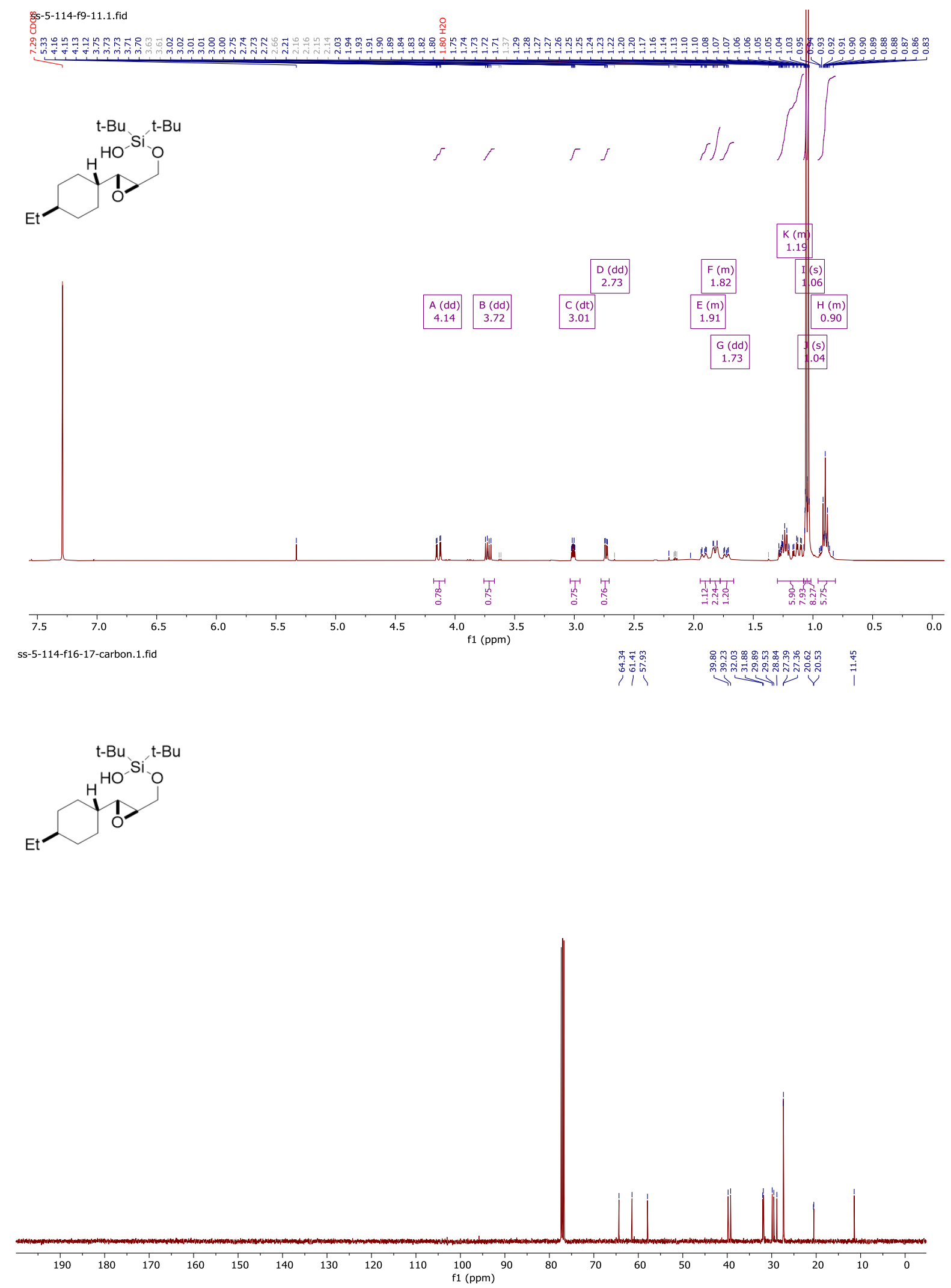
Compound $7\left({ }^{1} \mathrm{H} N M R: 400 \mathrm{MHz},{ }^{13} \mathrm{C}\left\{{ }^{1} \mathrm{H}\right\}\right.$ NMR: $\left.100 \mathrm{MHz}\right)$

ร్-5-110-f17-20.1.fid

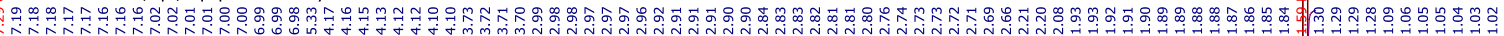

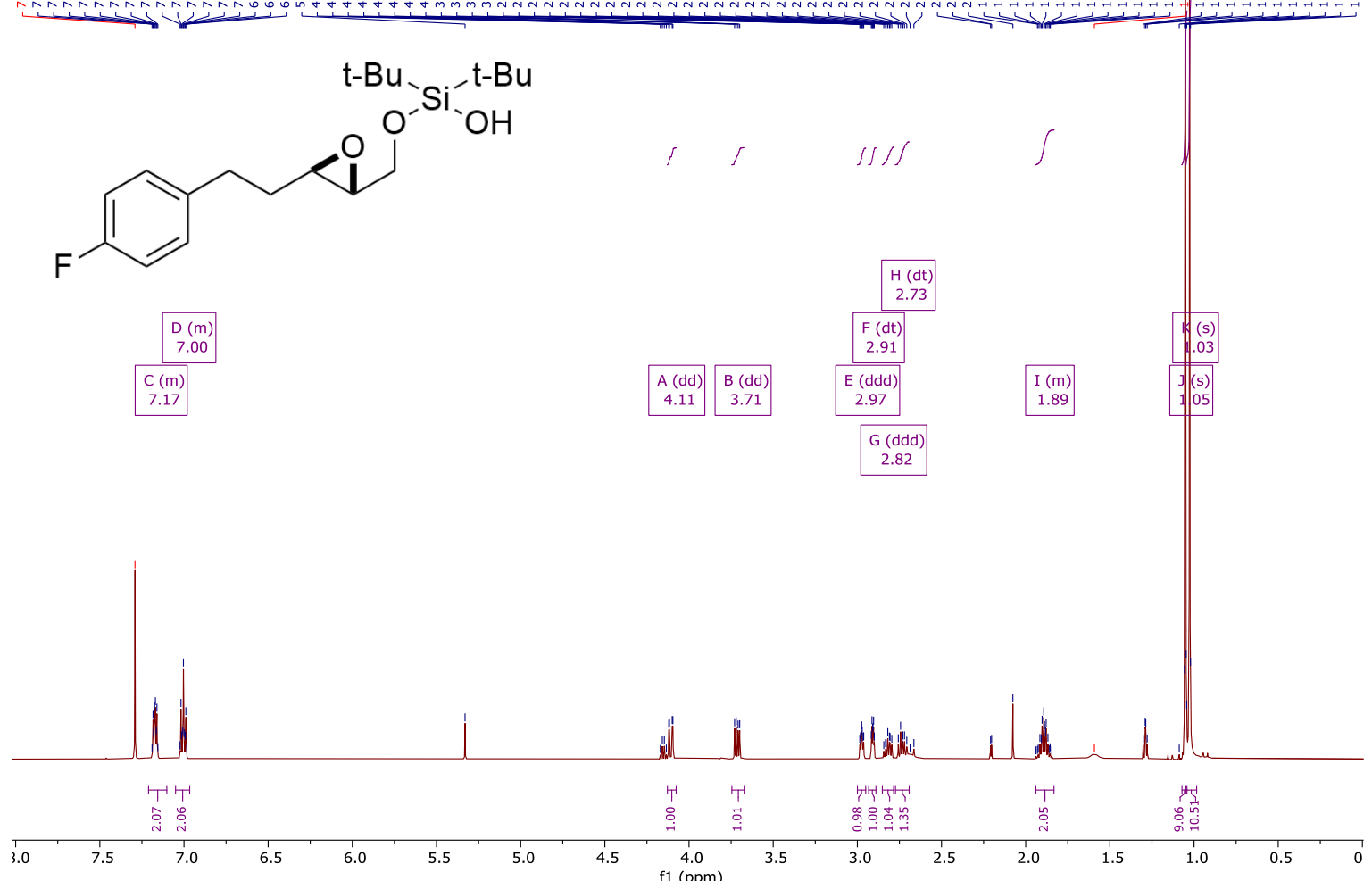

SS-5-110-CARBON.1.fid

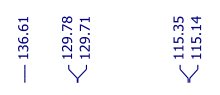

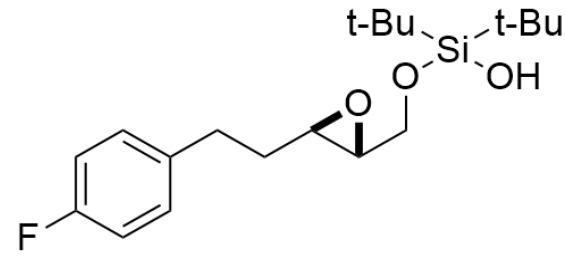
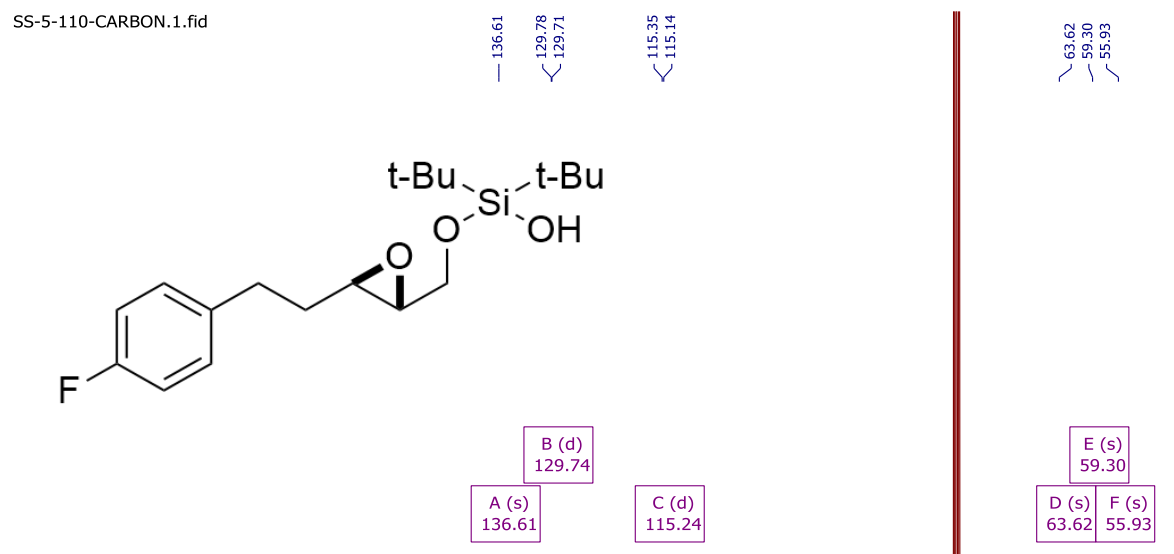

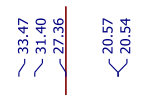

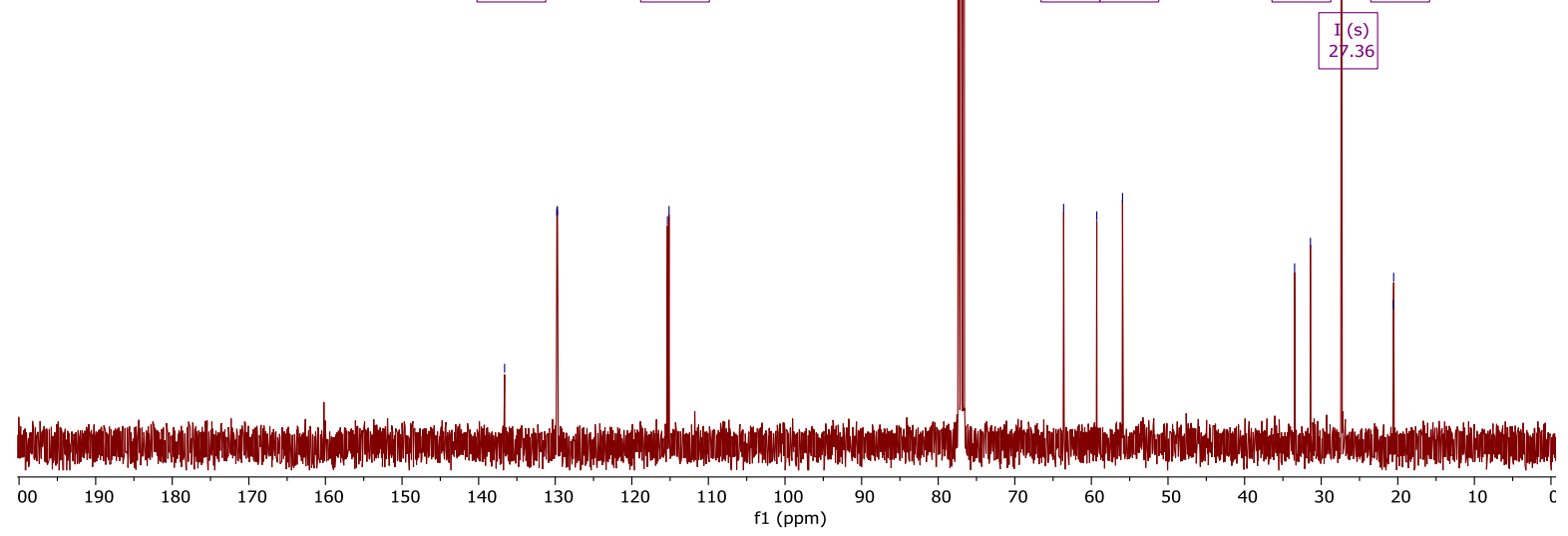


Compound $8\left({ }^{1} \mathrm{H}\right.$ NMR: $400 \mathrm{MHz},{ }^{13} \mathrm{C}\left\{{ }^{1} \mathrm{H}\right\}$ NMR: $100 \mathrm{MHz}$ )

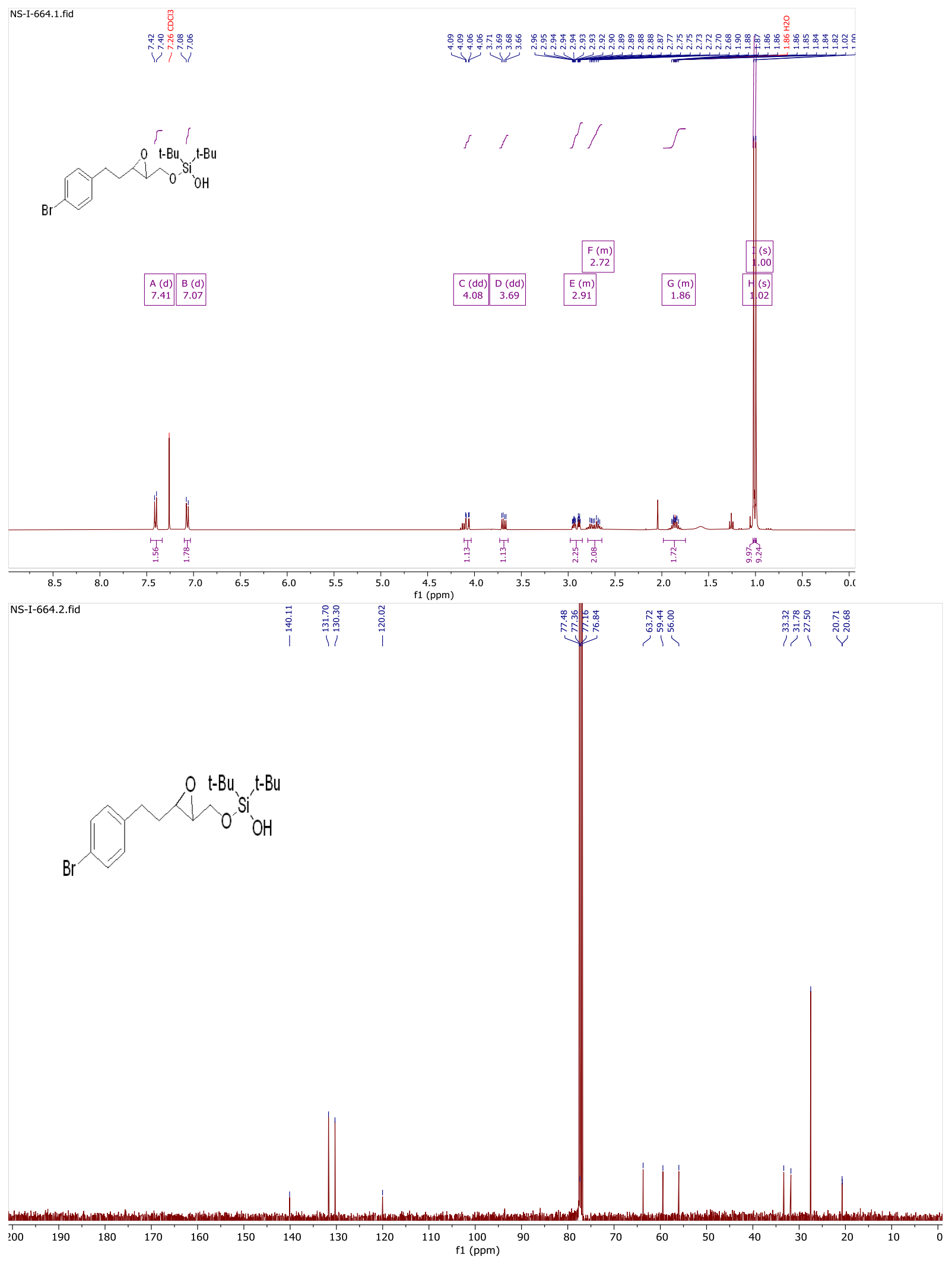


Compound 9 ( ${ }^{1} \mathrm{H}$ NMR: $400 \mathrm{MHz},{ }^{13} \mathrm{C}\left\{{ }^{1} \mathrm{H}\right\}$ NMR: $100 \mathrm{MHz}$ )

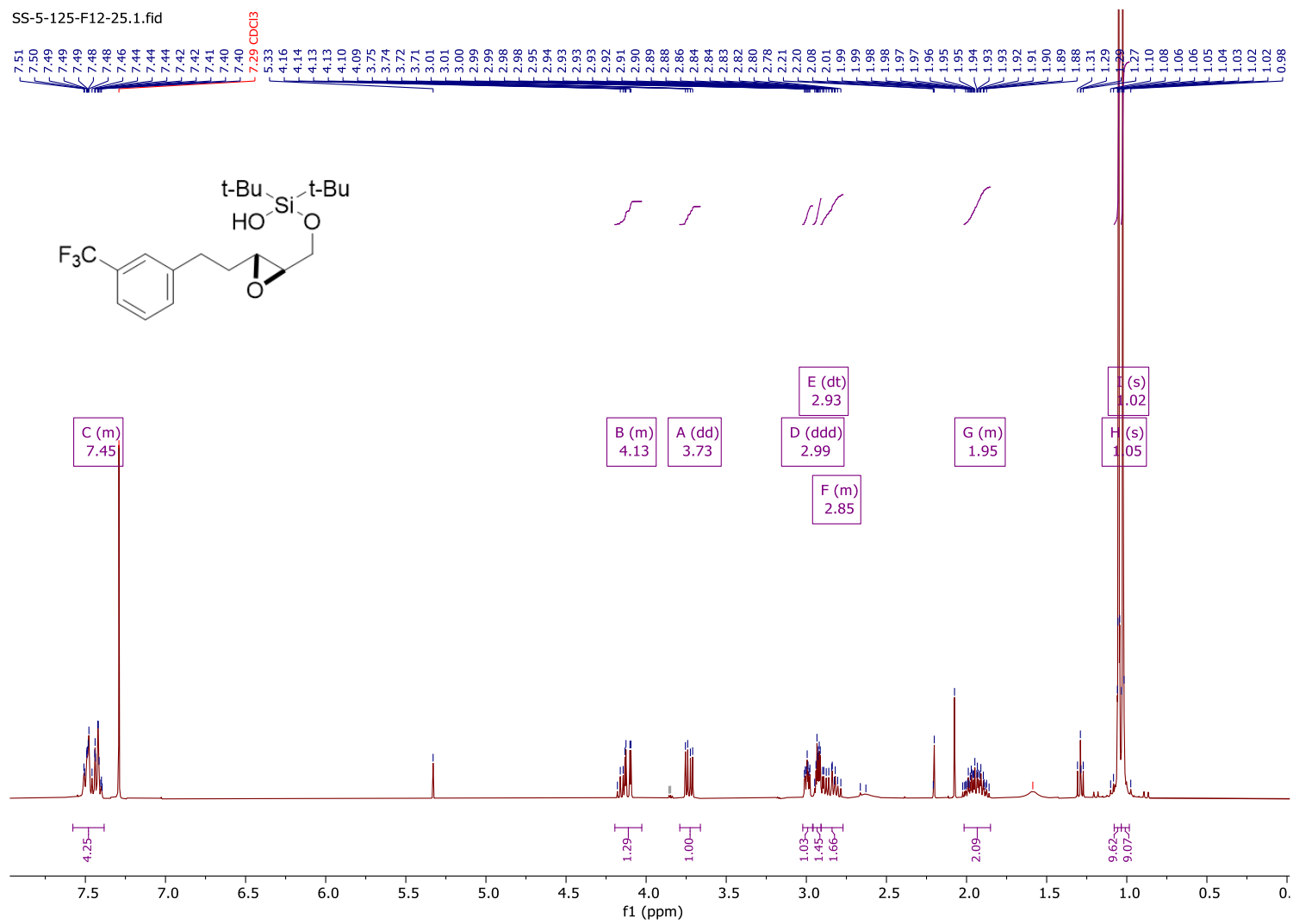

ss-5-125-carbon.1.fid

مै

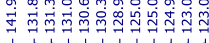

i

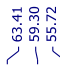

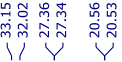
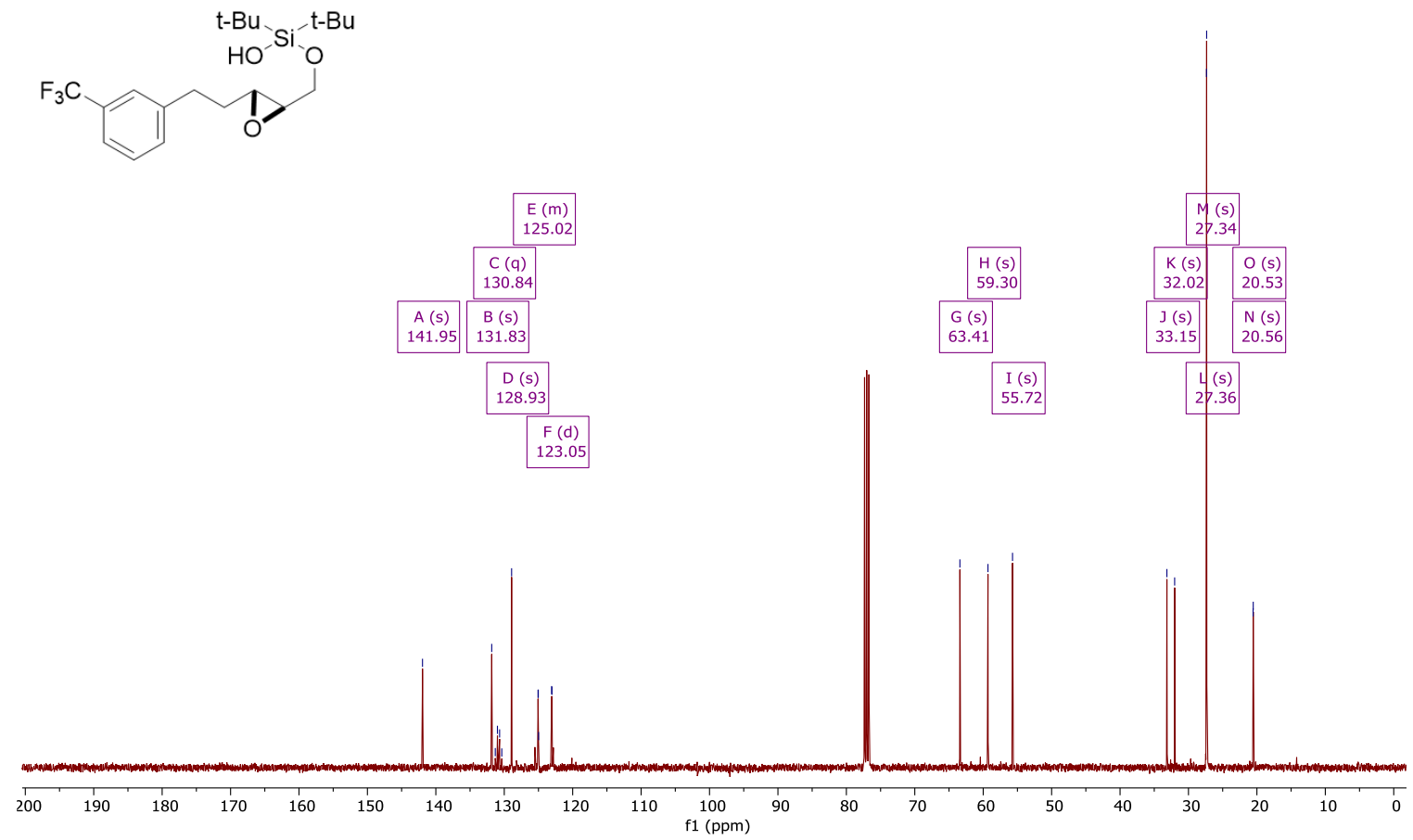
Compound 10 ( ${ }^{1} \mathrm{H}$ NMR: $400 \mathrm{MHz},{ }^{13} \mathrm{C}\left\{{ }^{1} \mathrm{H}\right\}$ NMR: $100 \mathrm{MHz}$ )
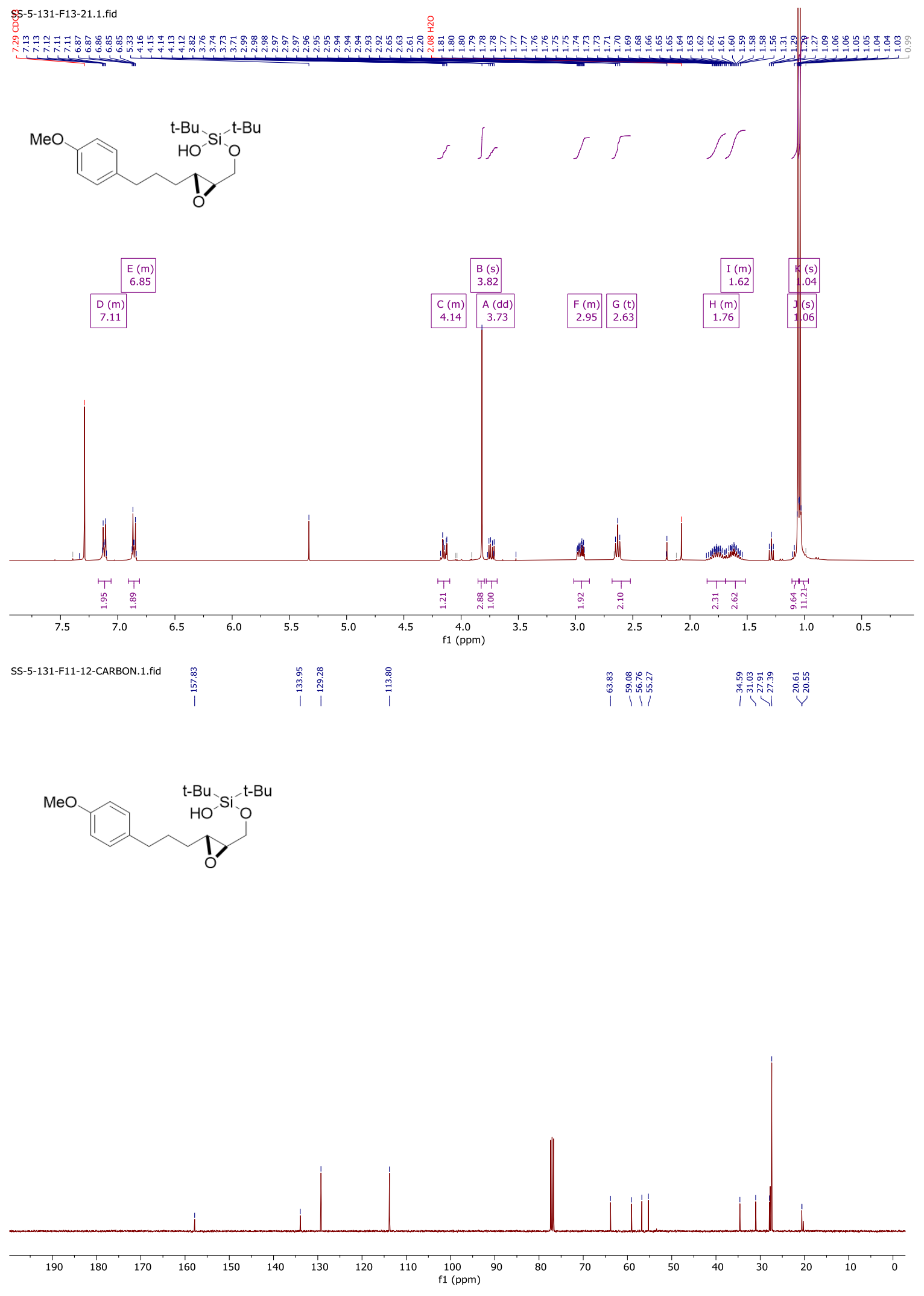

S68 


\section{Compound $11\left({ }^{1} \mathrm{H}\right.$ NMR: $400 \mathrm{MHz},{ }^{13} \mathrm{C}\left\{{ }^{1} \mathrm{H}\right\}$ NMR: $\left.100 \mathrm{MHz}\right)$}
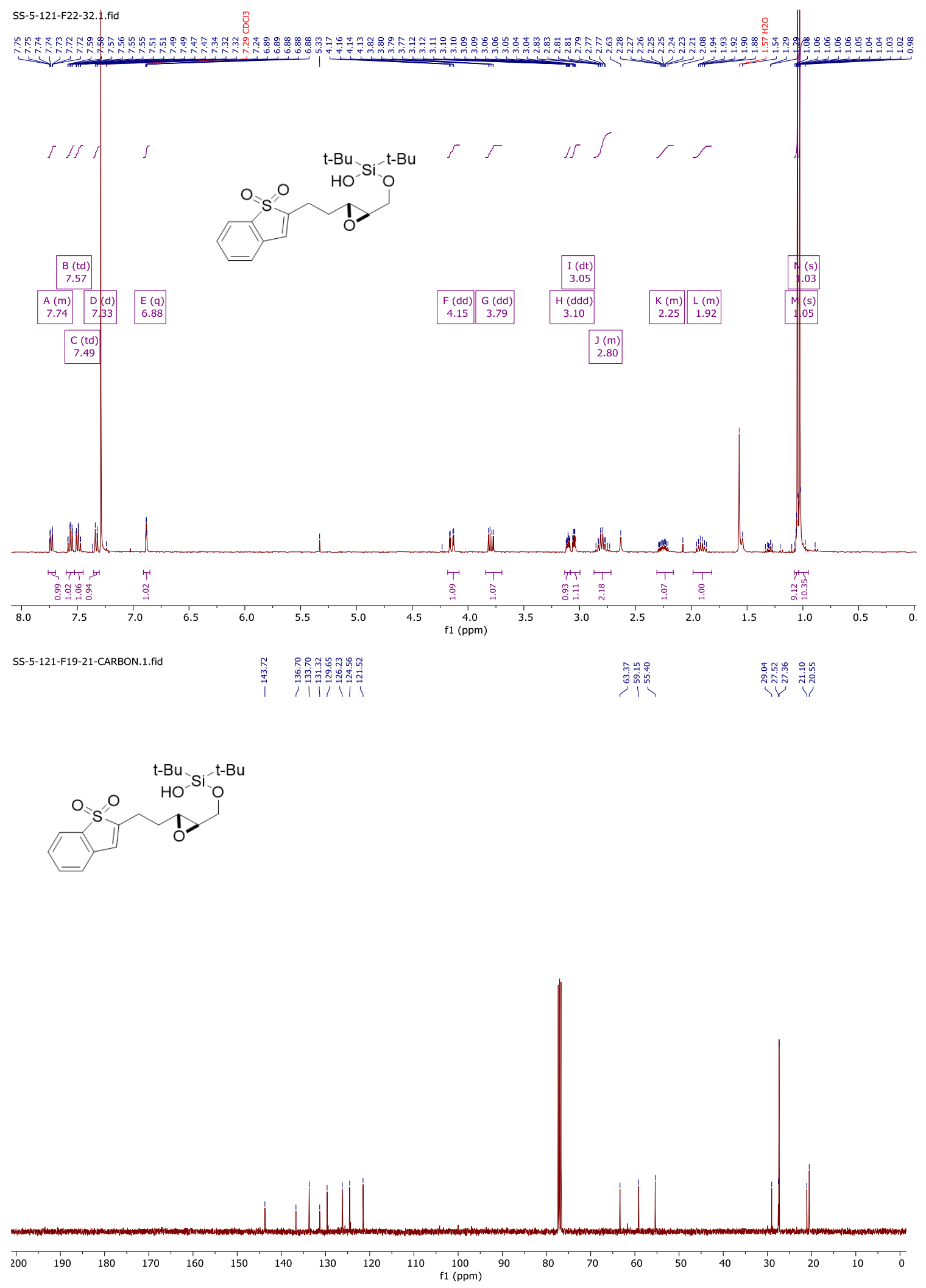
Compound $12\left({ }^{1} \mathrm{H}\right.$ NMR: $400 \mathrm{MHz},{ }^{13} \mathrm{C}\left\{{ }^{1} \mathrm{H}\right\}$ NMR: $\left.100 \mathrm{MHz}\right)$
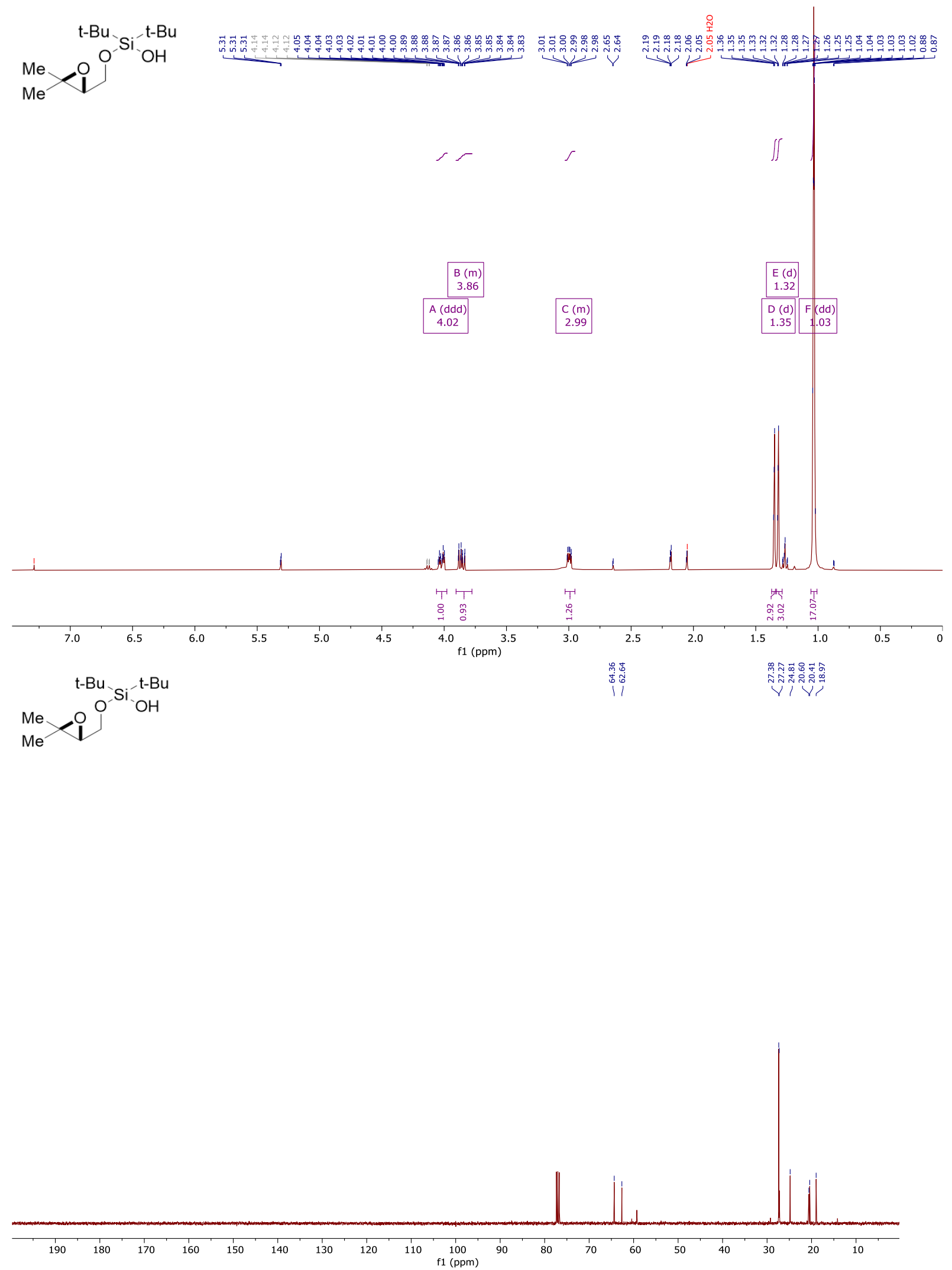
Compound $13\left({ }^{1} \mathrm{H}\right.$ NMR: $400 \mathrm{MHz},{ }^{13} \mathrm{C}\left\{{ }^{1} \mathrm{H}\right\}$ NMR: $\left.100 \mathrm{MHz}\right)$
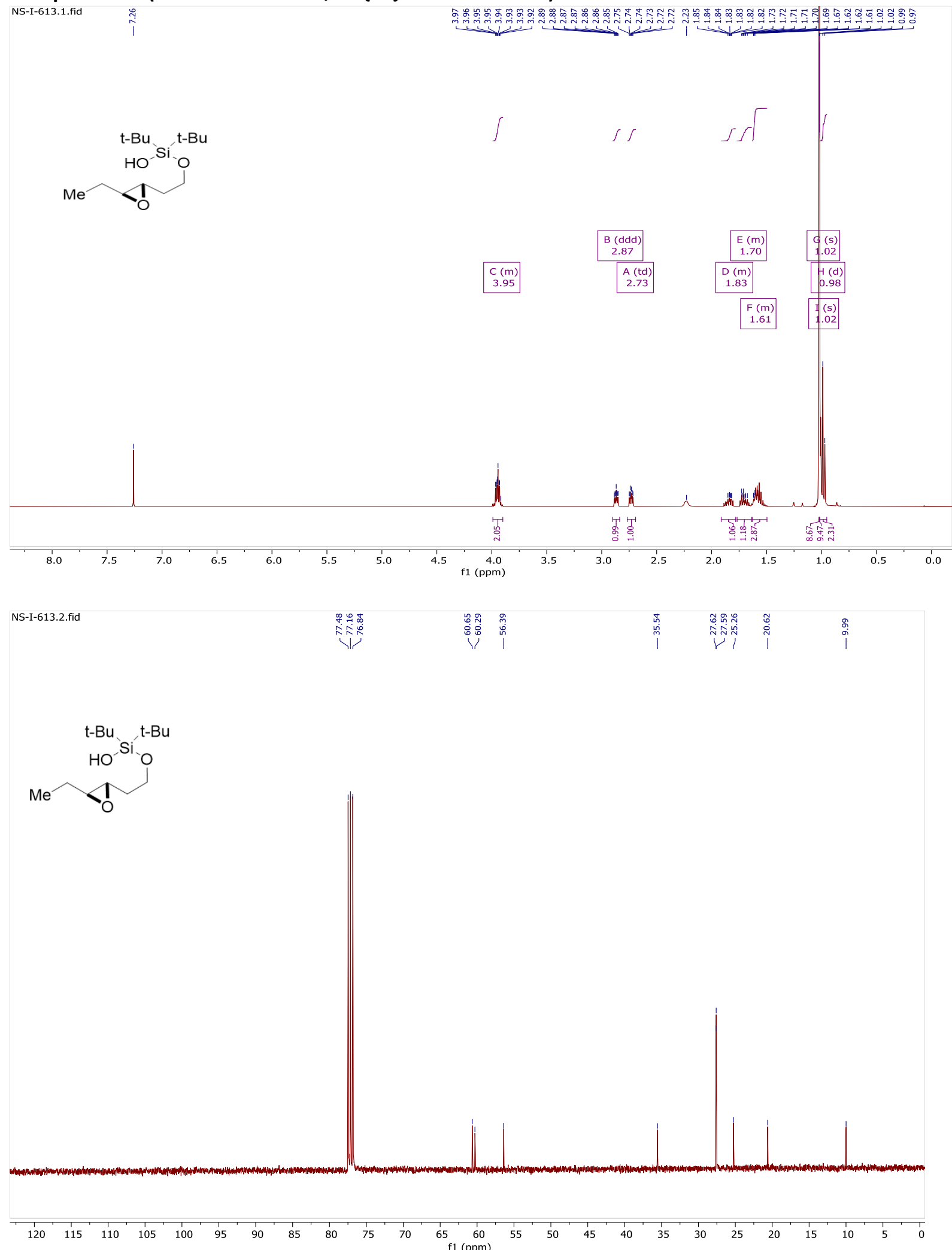
Compound $14\left({ }^{1} \mathrm{H}\right.$ NMR: $400 \mathrm{MHz},{ }^{13} \mathrm{C}\left\{{ }^{1} \mathrm{H}\right\}$ NMR: $\left.100 \mathrm{MHz}\right)$
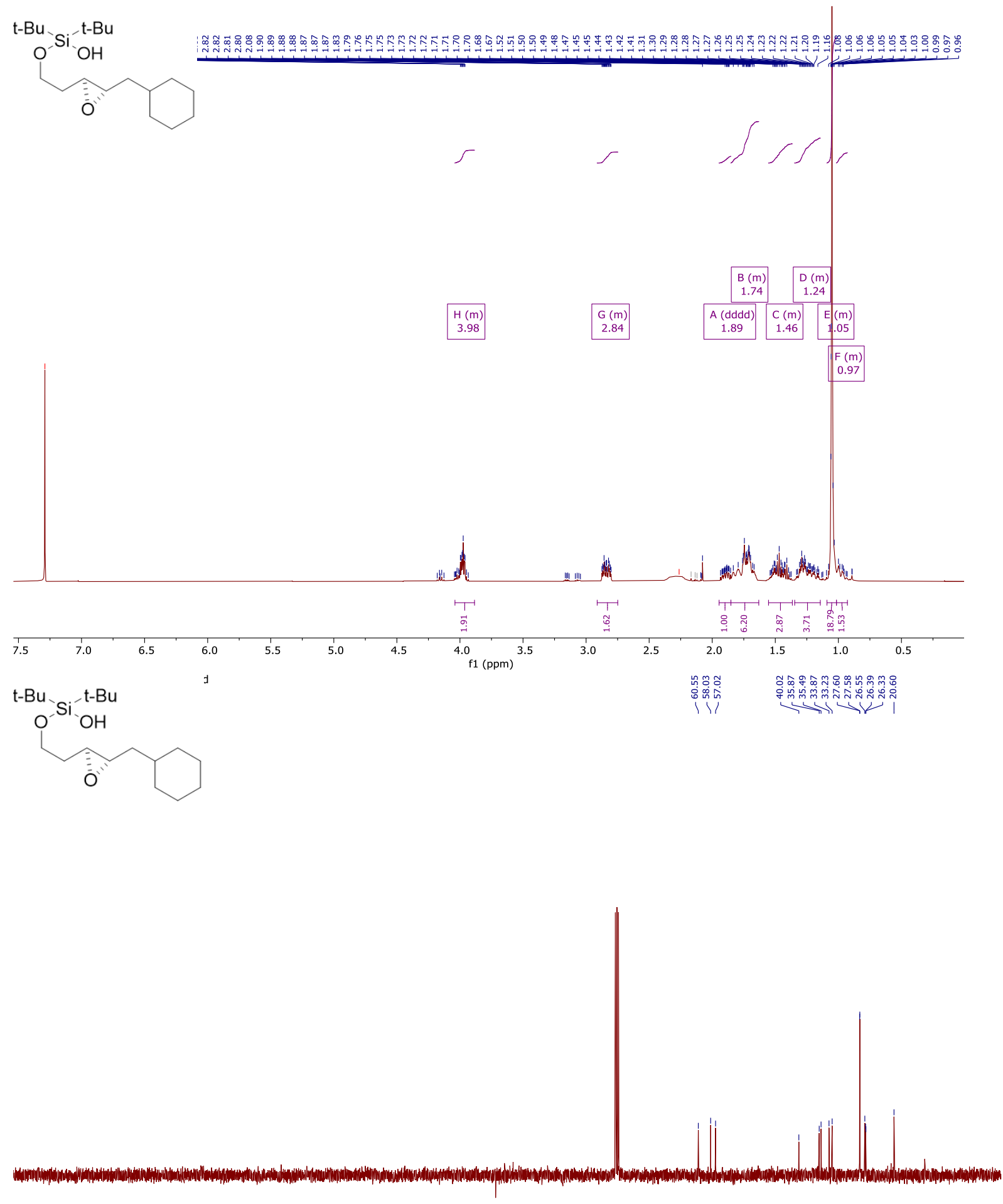

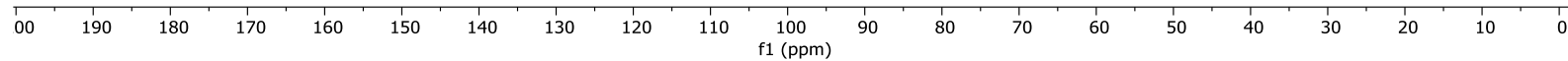


Compound $15\left({ }^{1} \mathrm{H}\right.$ NMR: $400 \mathrm{MHz},{ }^{13} \mathrm{C}\left\{{ }^{1} \mathrm{H}\right\}$ NMR: $\left.100 \mathrm{MHz}\right)$
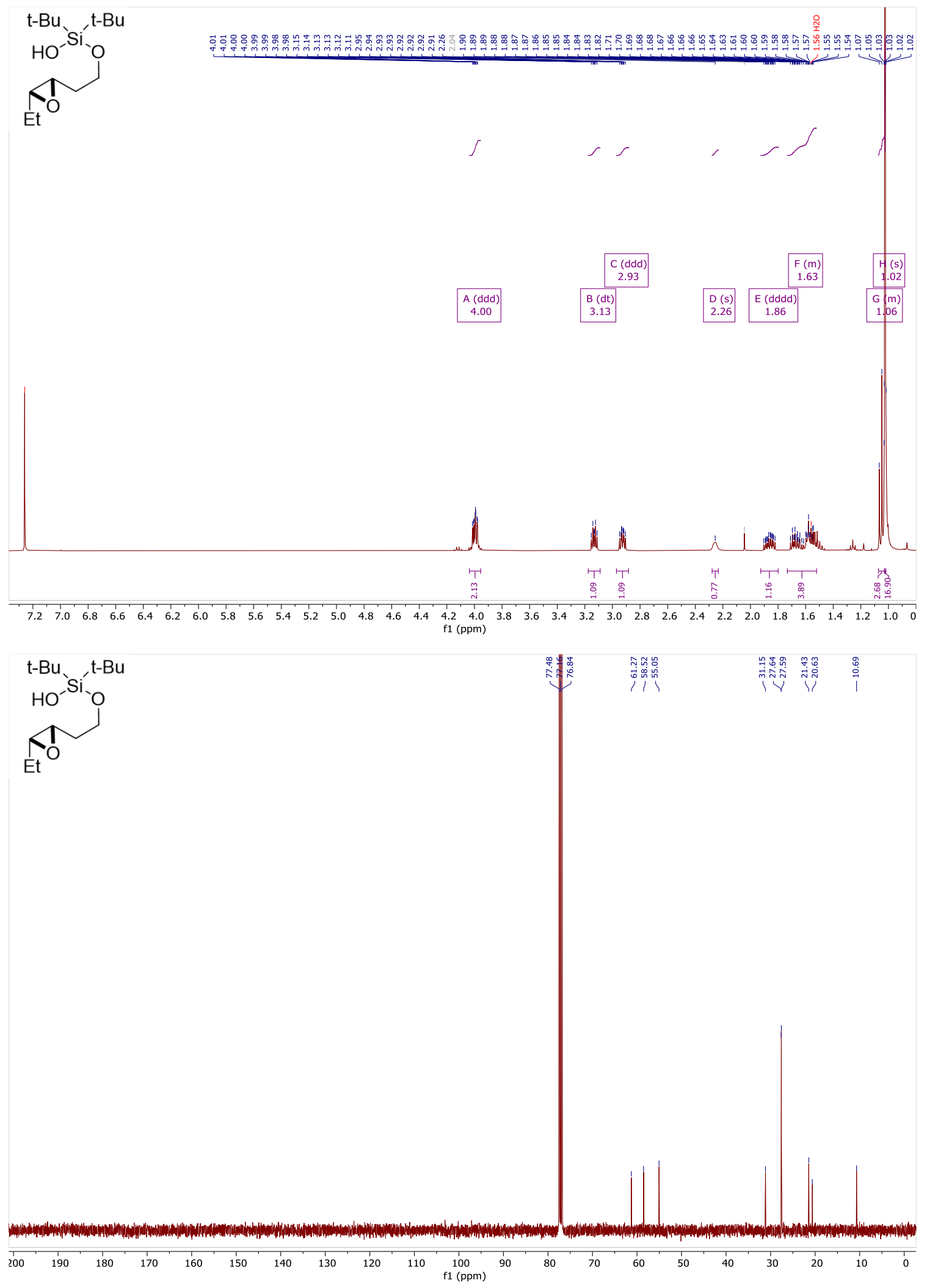
Compound $16\left({ }^{1} \mathrm{H}\right.$ NMR: $400 \mathrm{MHz},{ }^{13} \mathrm{C}\left\{{ }^{1} \mathrm{H}\right\}$ NMR: $\left.100 \mathrm{MHz}\right)$

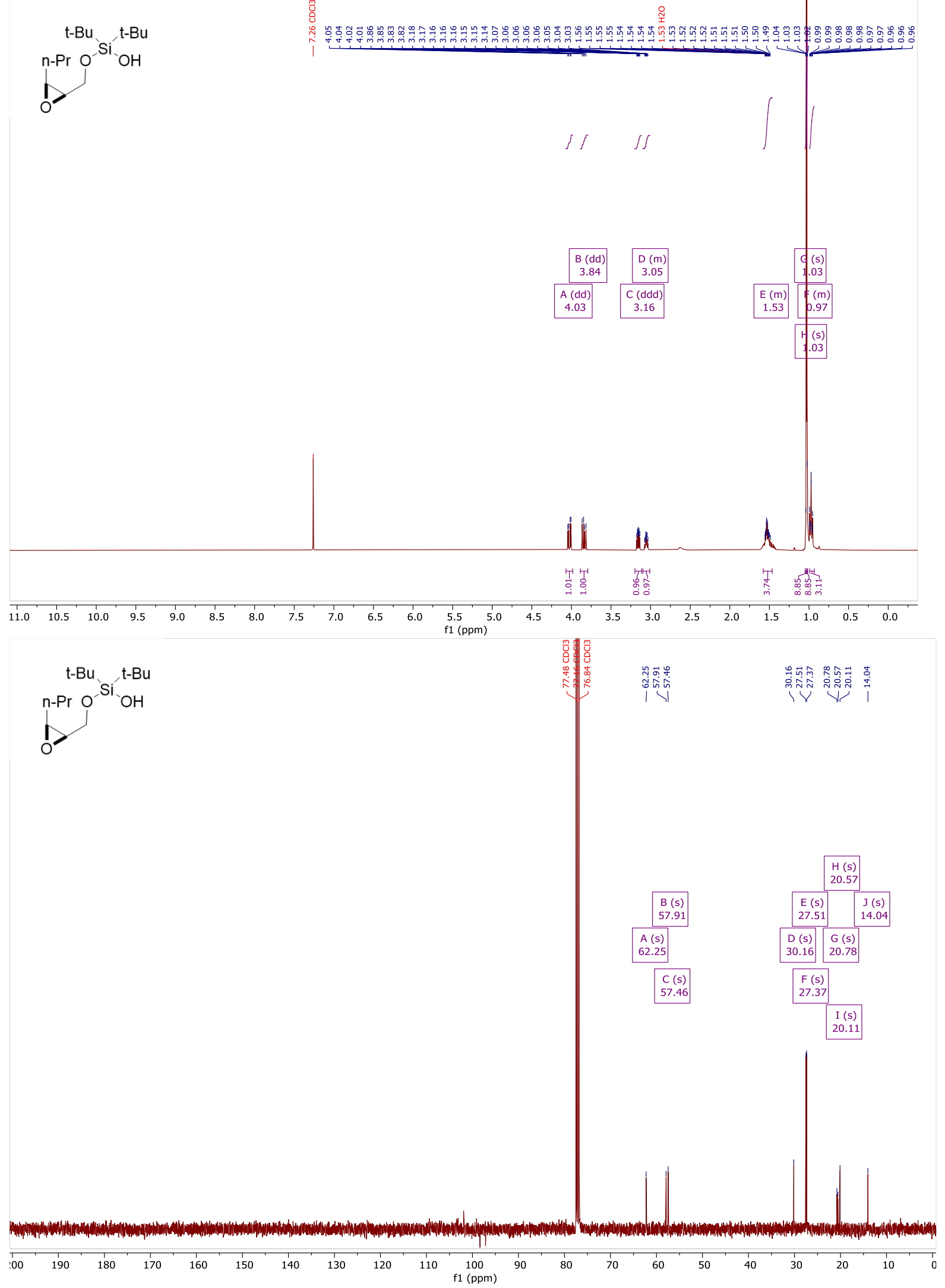


Compound $17\left({ }^{1} \mathrm{H}\right.$ NMR: $400 \mathrm{MHz},{ }^{13} \mathrm{C}\left\{{ }^{1} \mathrm{H}\right\}$ NMR: $100 \mathrm{MHz}$ )
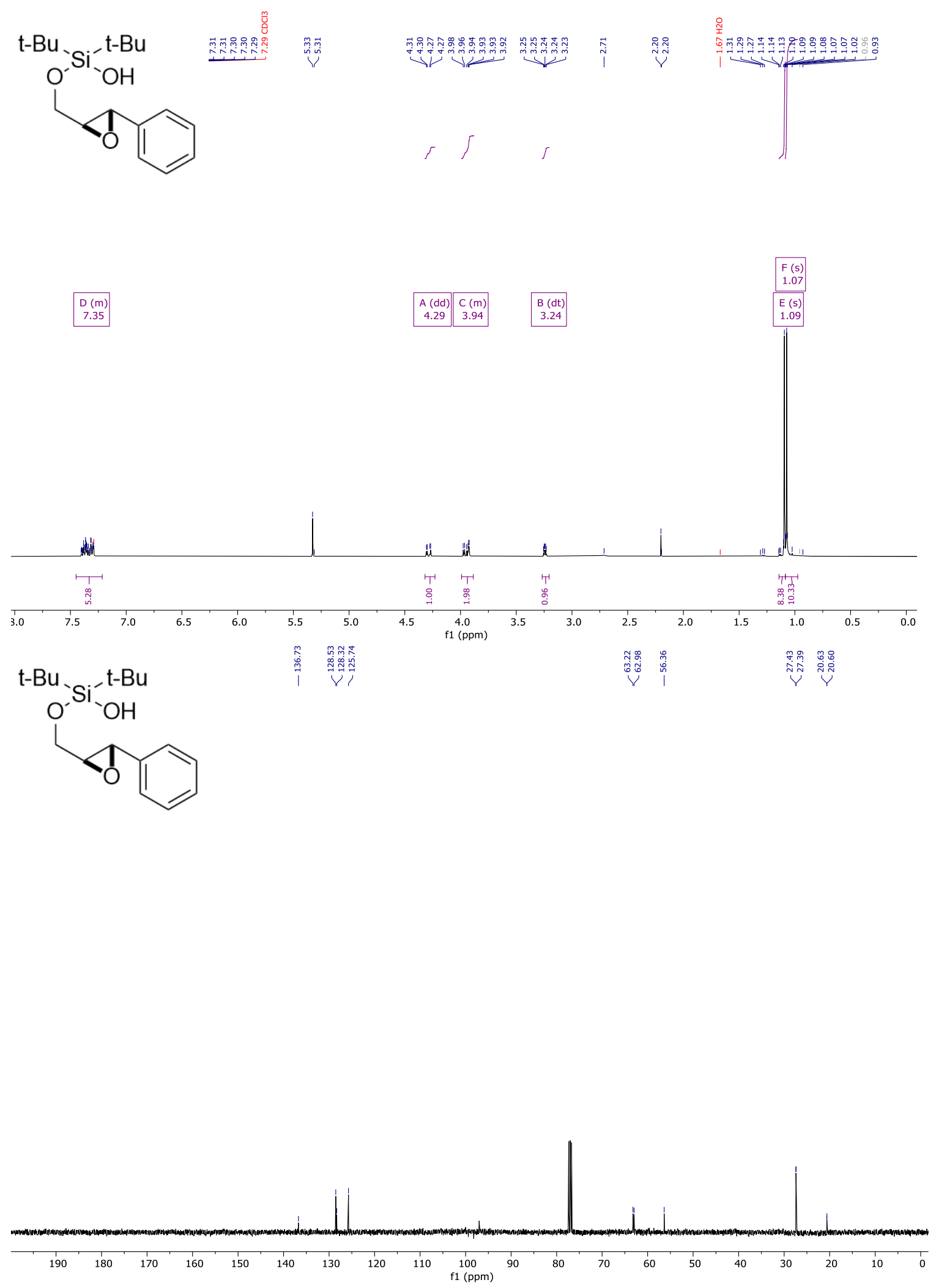


\section{Compound $18\left({ }^{1} \mathrm{H}\right.$ NMR: $400 \mathrm{MHz},{ }^{13} \mathrm{C}\left\{{ }^{1} \mathrm{H}\right\}$ NMR: $\left.100 \mathrm{MHz}\right)$}
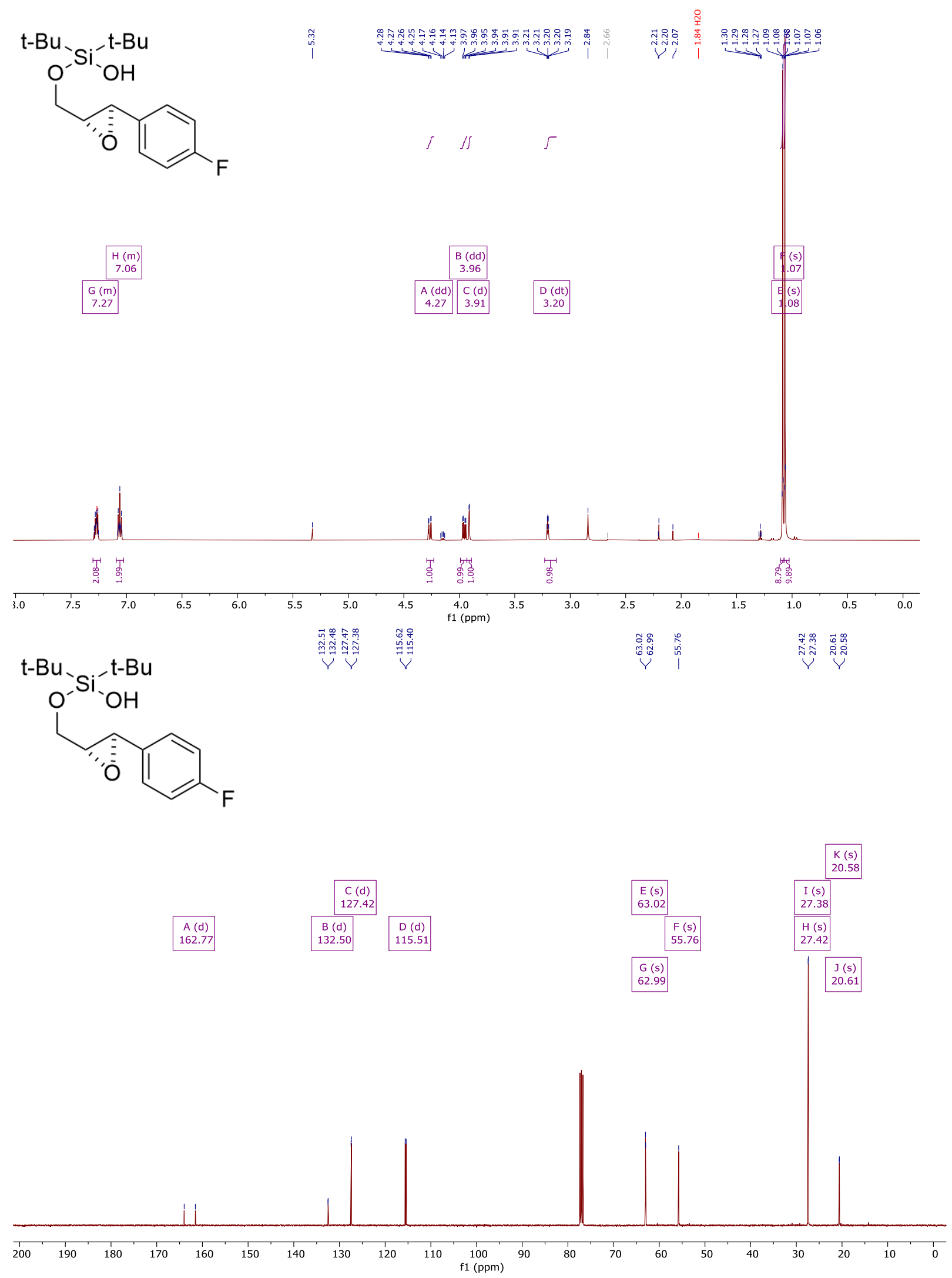
Compound $19\left({ }^{1} \mathrm{H}\right.$ NMR: $400 \mathrm{MHz},{ }^{13} \mathrm{C}\left\{{ }^{1} \mathrm{H}\right\}$ NMR: $\left.100 \mathrm{MHz}\right)$
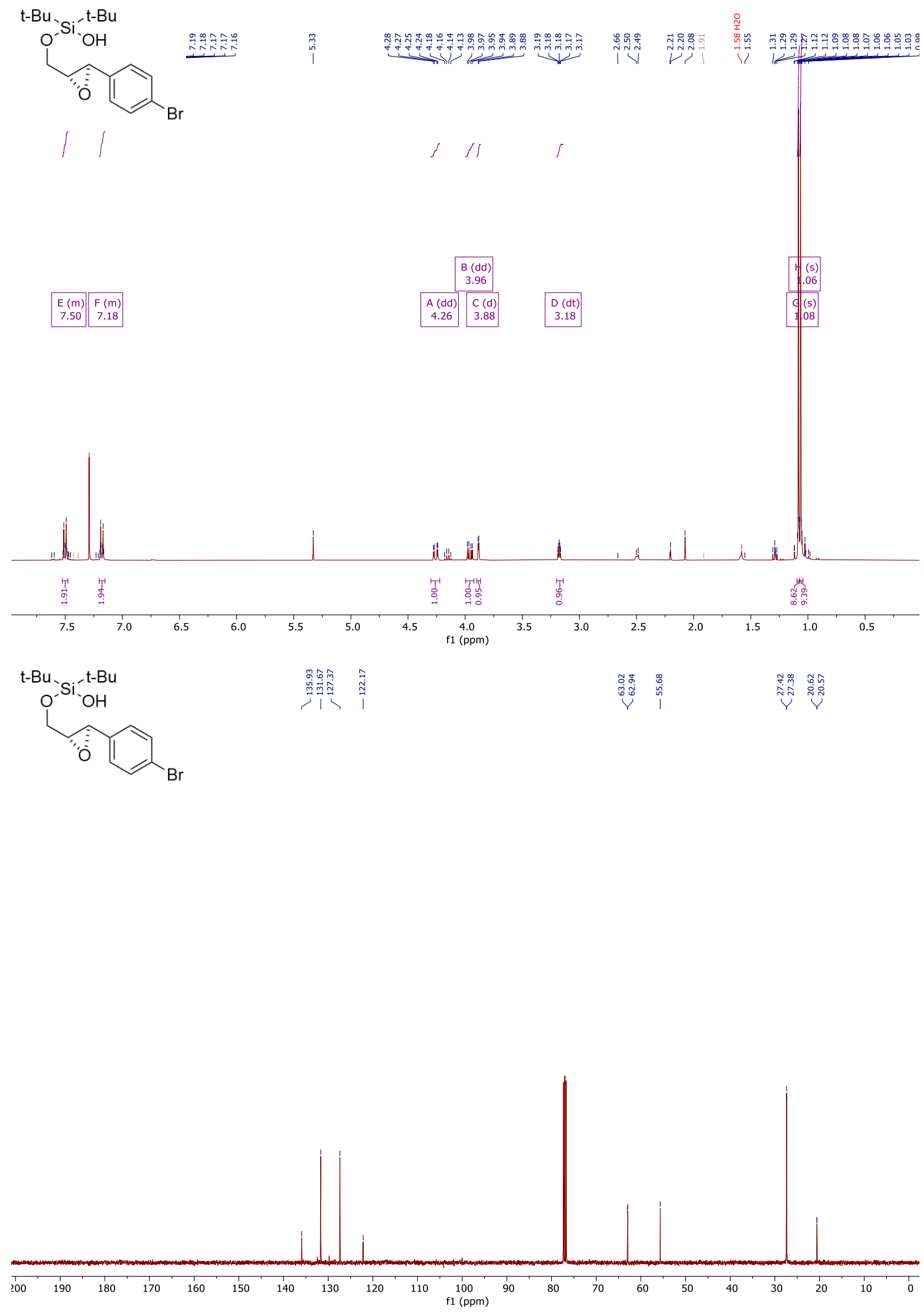
Compound 20 ( ${ }^{1} \mathrm{H}$ NMR: $400 \mathrm{MHz},{ }^{13} \mathrm{C}\left\{{ }^{1} \mathrm{H}\right\}$ NMR: $100 \mathrm{MHz}$ )

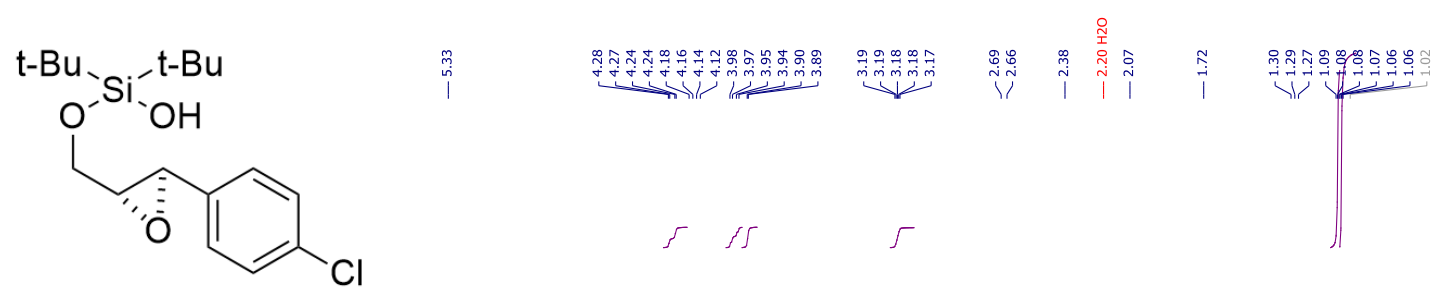

$$
\begin{array}{|c|c|c|c|c|}
\hline F(m) \\
7.23 \\
\hline E(m) \\
7.34 \\
\hline
\end{array}
$$
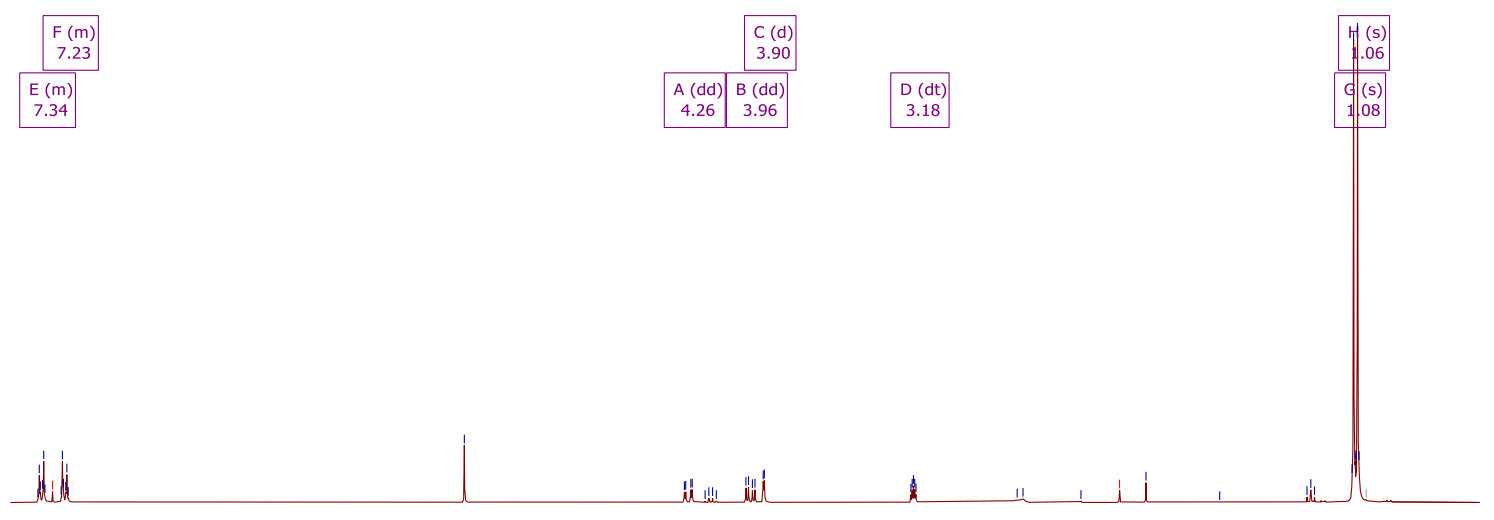

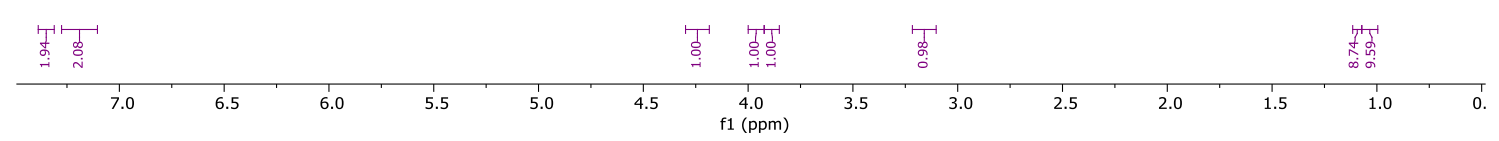<smiles>CC(C)(C)C[Si](O)(OC[C@H]1O[C@@H]1c1ccc(Cl)cc1)C(C)(C)C</smiles>

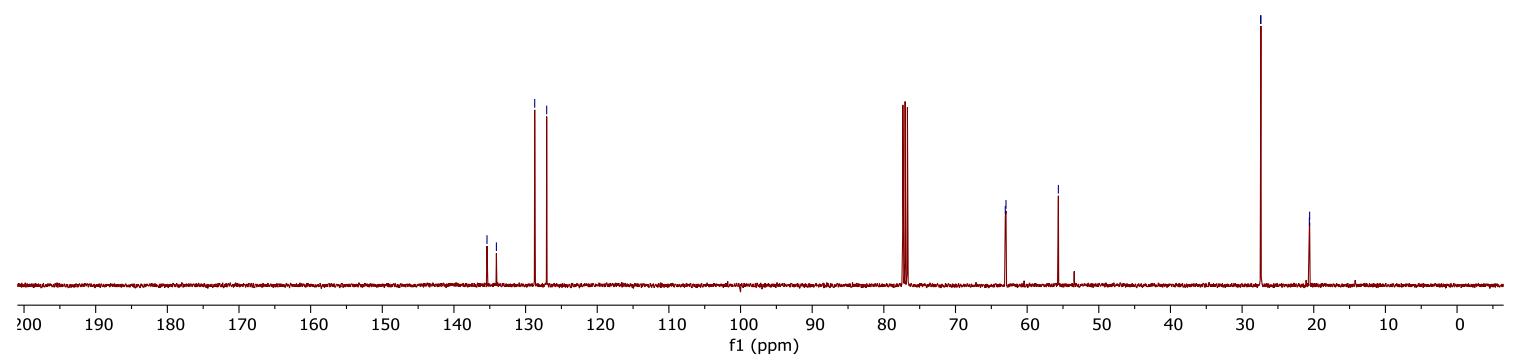


Compound $21\left({ }^{1} \mathrm{H}\right.$ NMR: $400 \mathrm{MHz},{ }^{13} \mathrm{C}\left\{{ }^{1} \mathrm{H}\right\}$ NMR: $\left.100 \mathrm{MHz}\right)$
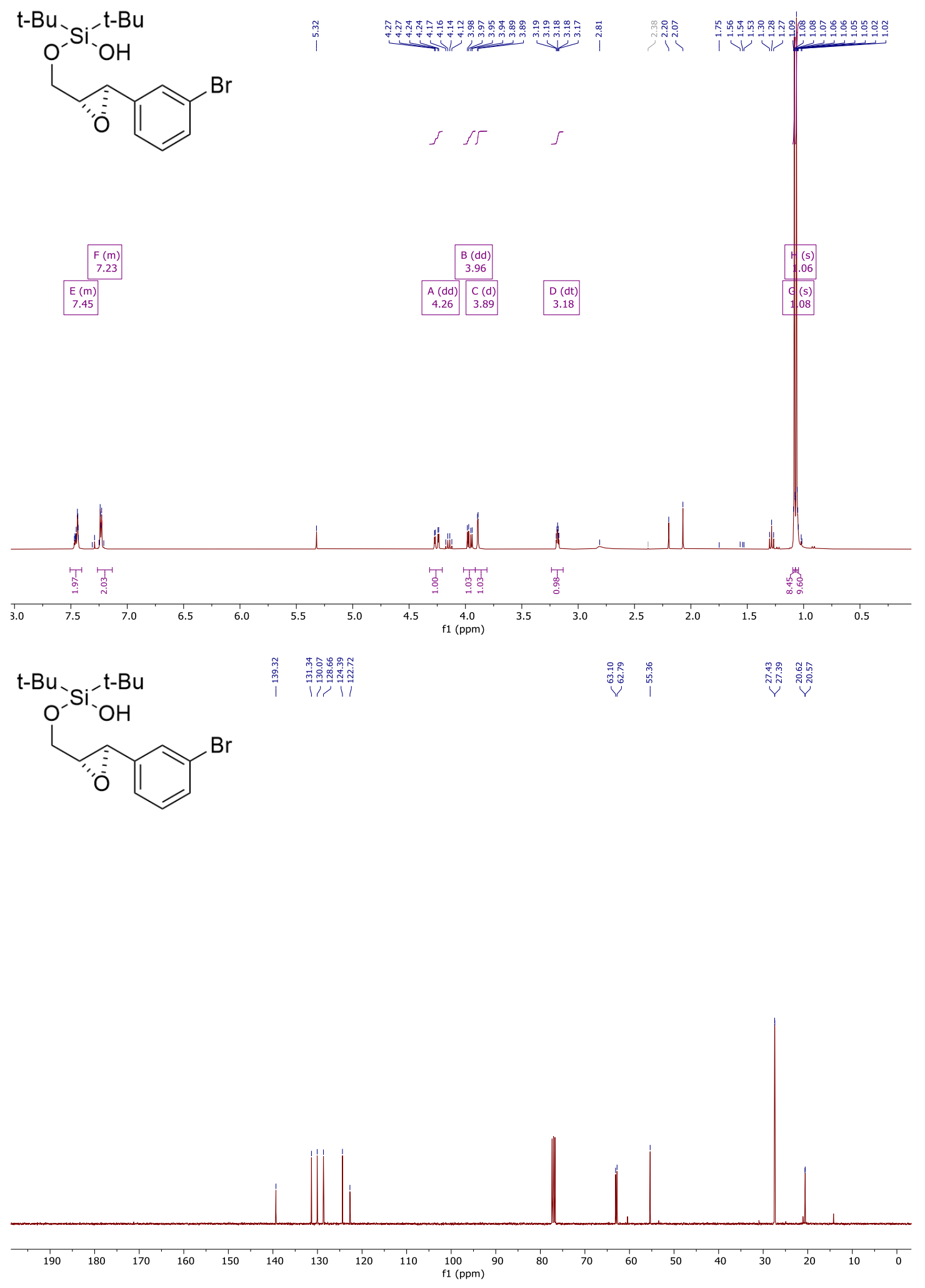
Compound $22\left({ }^{1} \mathrm{H}\right.$ NMR: $400 \mathrm{MHz},{ }^{13} \mathrm{C}\left\{{ }^{1} \mathrm{H}\right\}$ NMR: $\left.100 \mathrm{MHz}\right)$

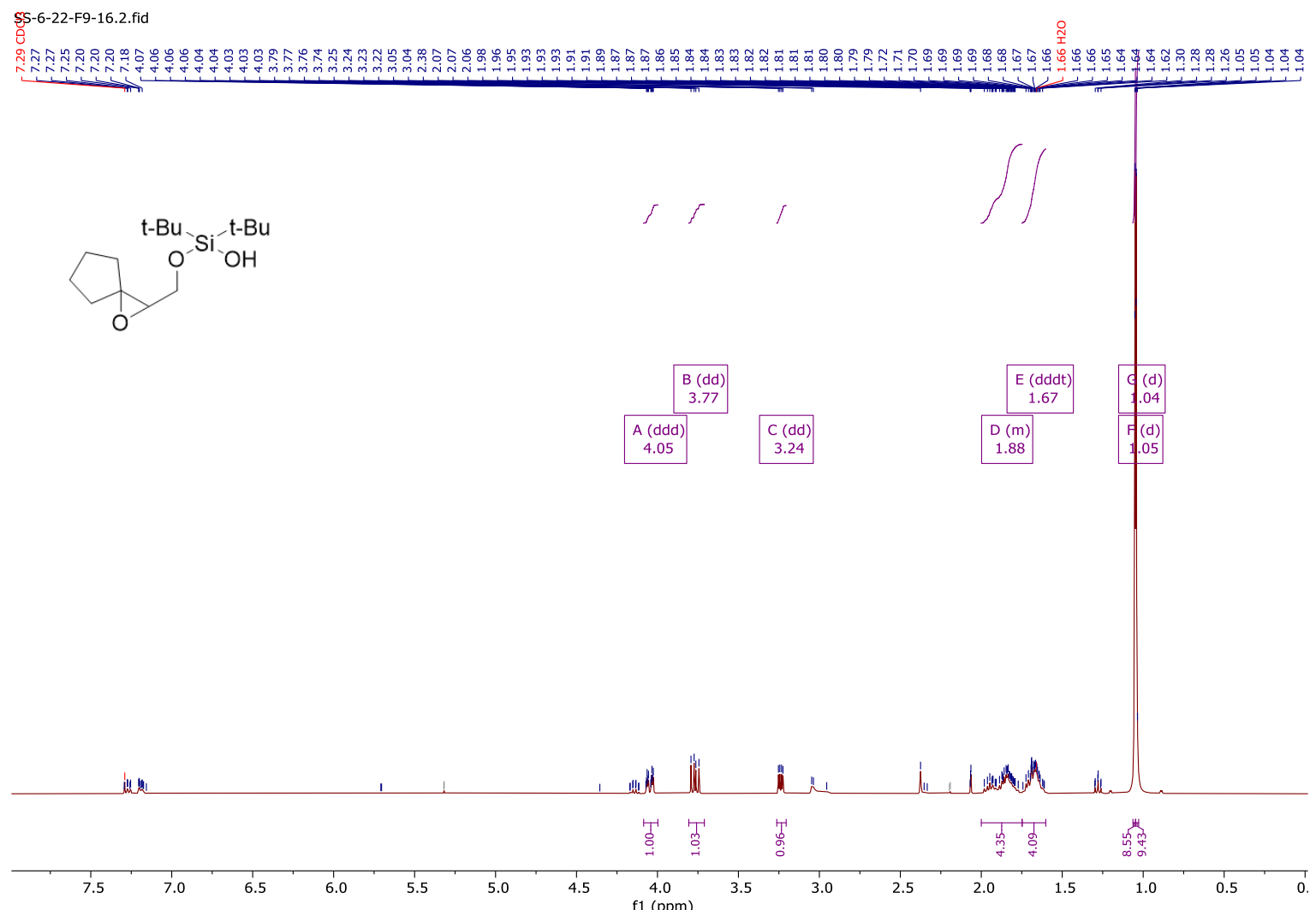

SS-6-22-F9-16-CARBON.2.fid

$a_{0}^{\mathrm{t}-\mathrm{Bu}} \mathrm{O}_{-\mathrm{Si}}^{\mathrm{-t}-\mathrm{Bu}}$

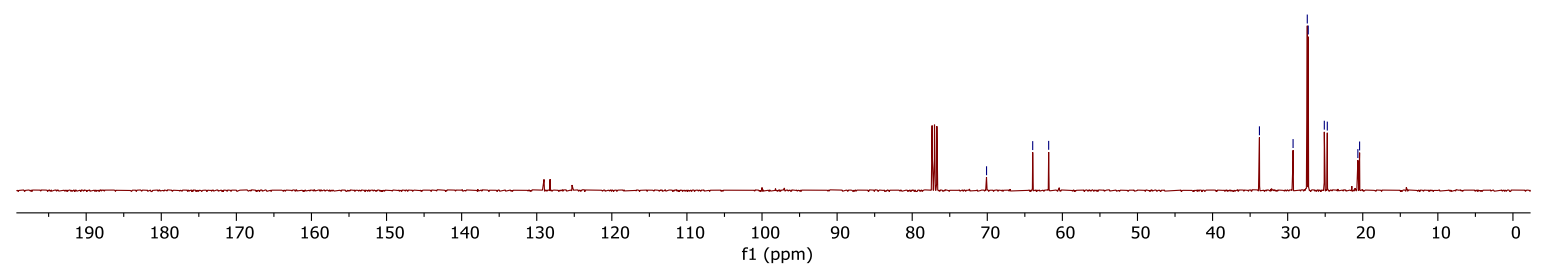


Compound $23\left({ }^{1} \mathrm{H}\right.$ NMR: $400 \mathrm{MHz},{ }^{13} \mathrm{C}\left\{{ }^{1} \mathrm{H}\right\}$ NMR: $\left.100 \mathrm{MHz}\right)$

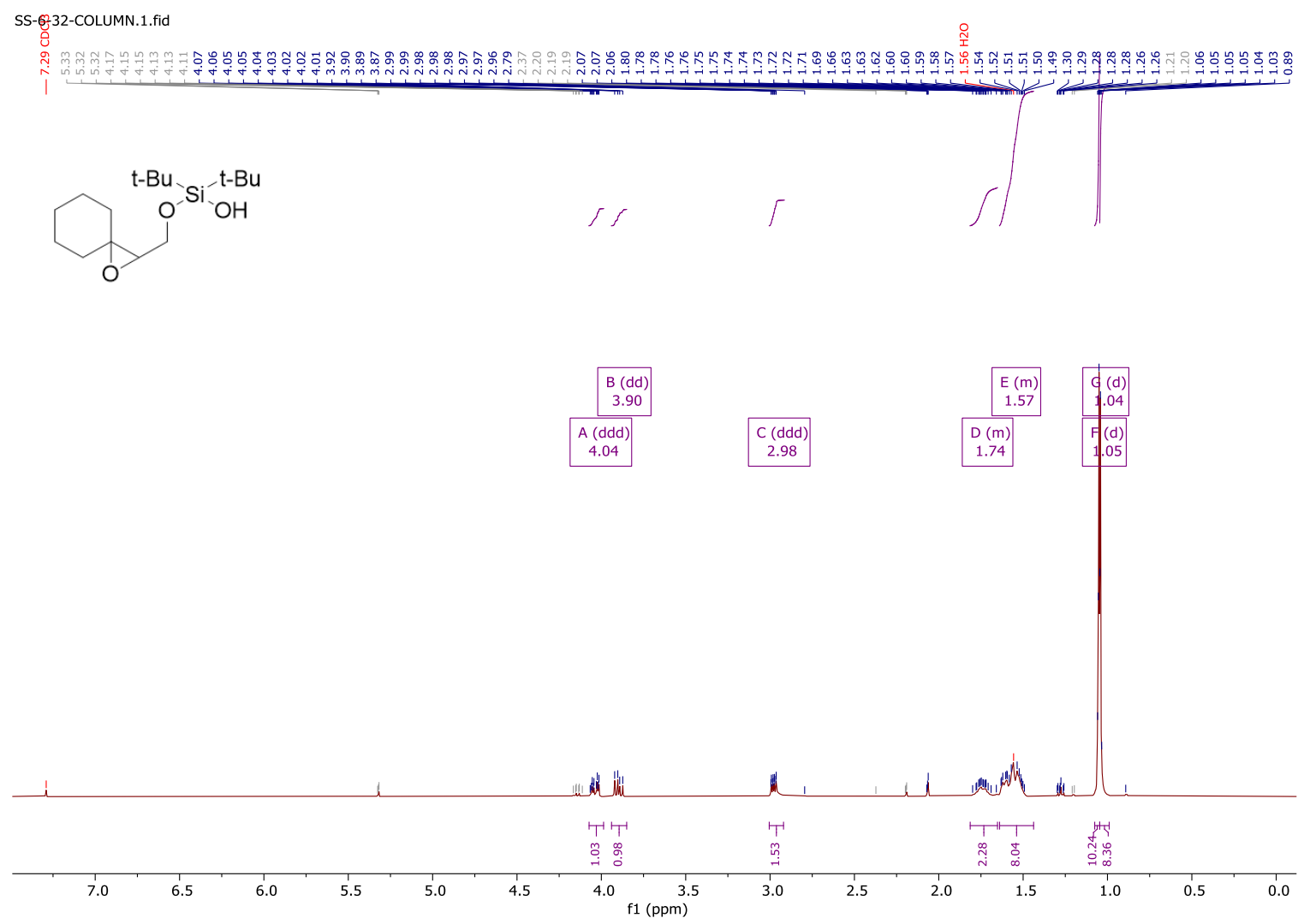

SS-6-32-COLUMN-CARBON.1.fid

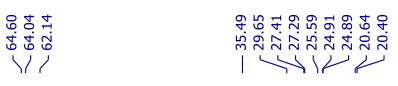

$\overbrace{0}^{\mathrm{t}-\mathrm{Bu}} \mathrm{O}^{-\mathrm{Si}} \mathrm{OH}^{\mathrm{t}-\mathrm{Bu}}$

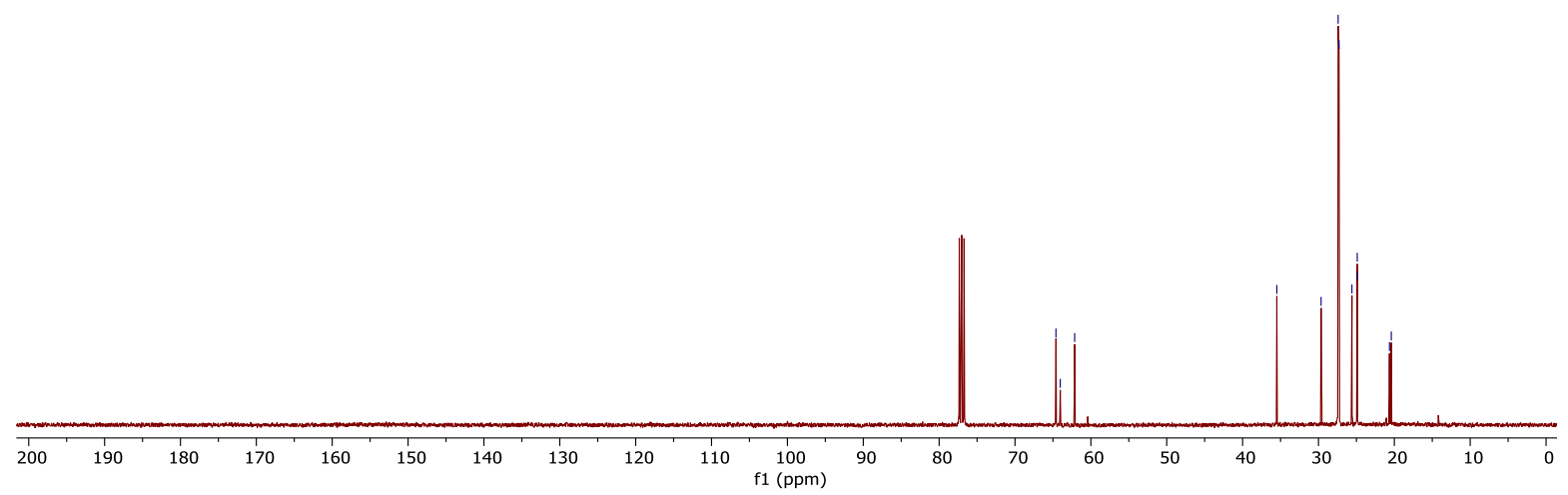


Compound $24\left({ }^{1} \mathrm{H}\right.$ NMR: $400 \mathrm{MHz},{ }^{13} \mathrm{C}\left\{{ }^{1} \mathrm{H}\right\}$ NMR: $\left.100 \mathrm{MHz}\right)$

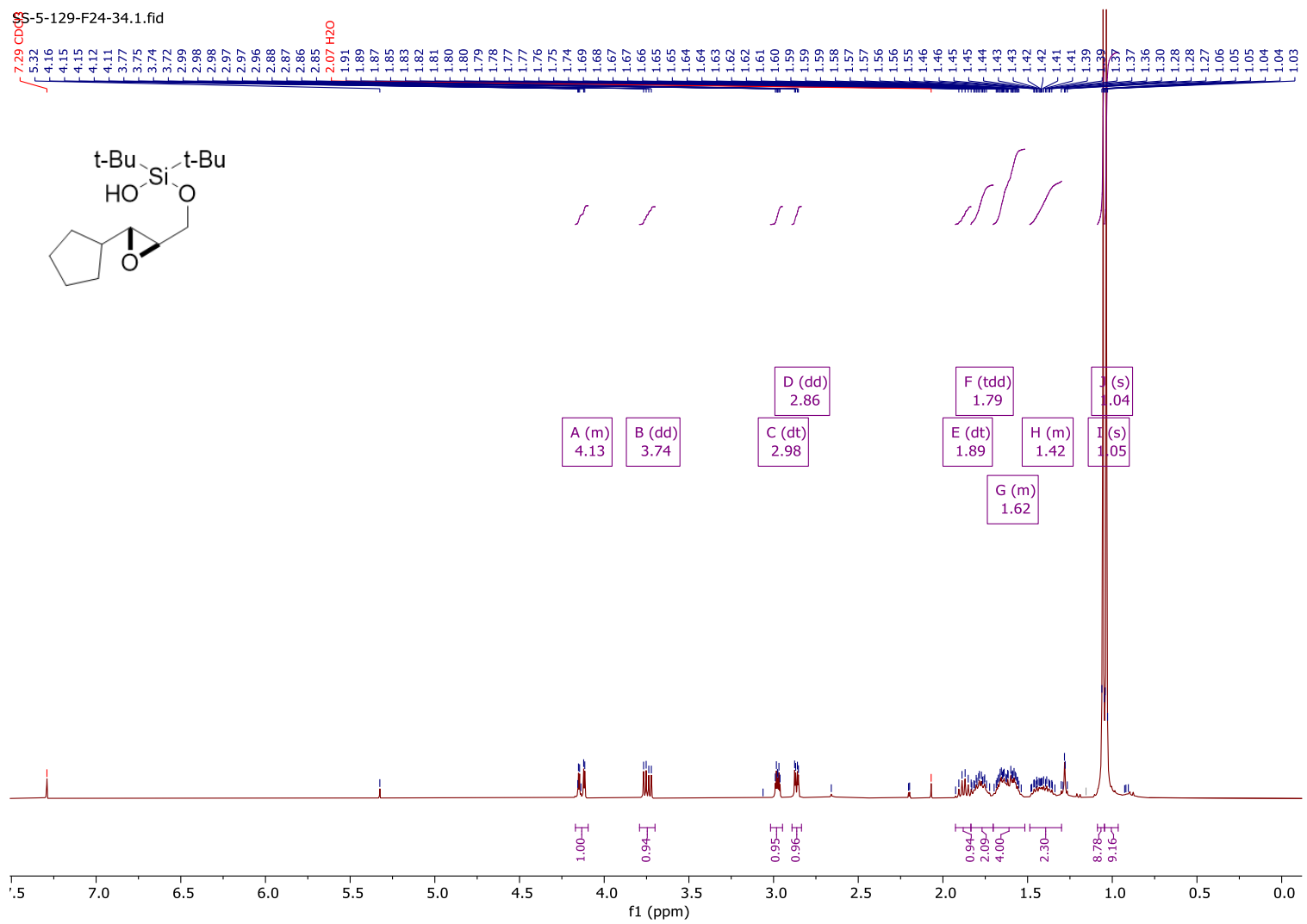

SS-5-129-F24-34-CARBON.1.fid
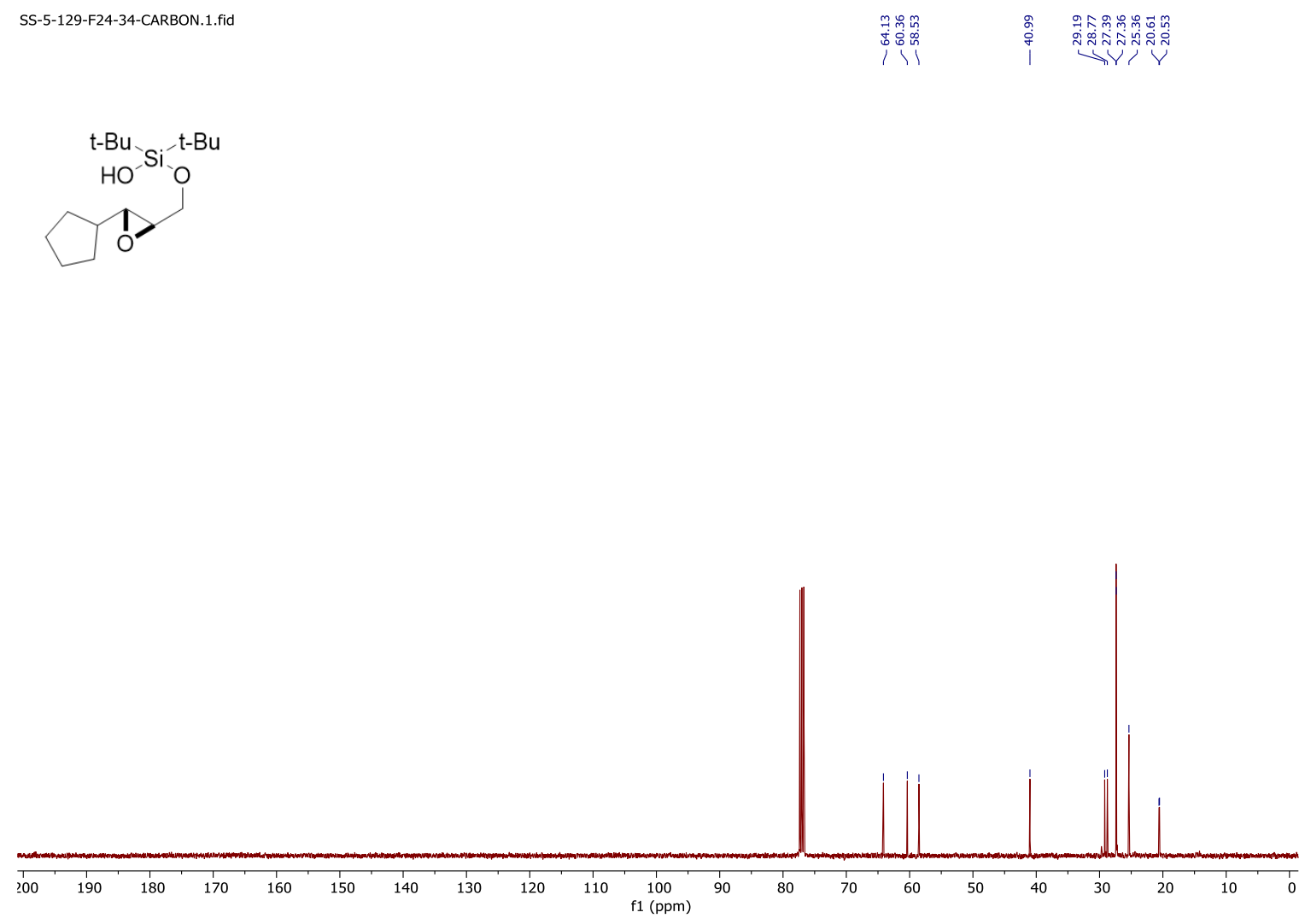
Compound $25\left({ }^{1} \mathrm{H}\right.$ NMR: $400 \mathrm{MHz},{ }^{13} \mathrm{C}\left\{{ }^{1} \mathrm{H}\right\}$ NMR: $\left.100 \mathrm{MHz}\right)$

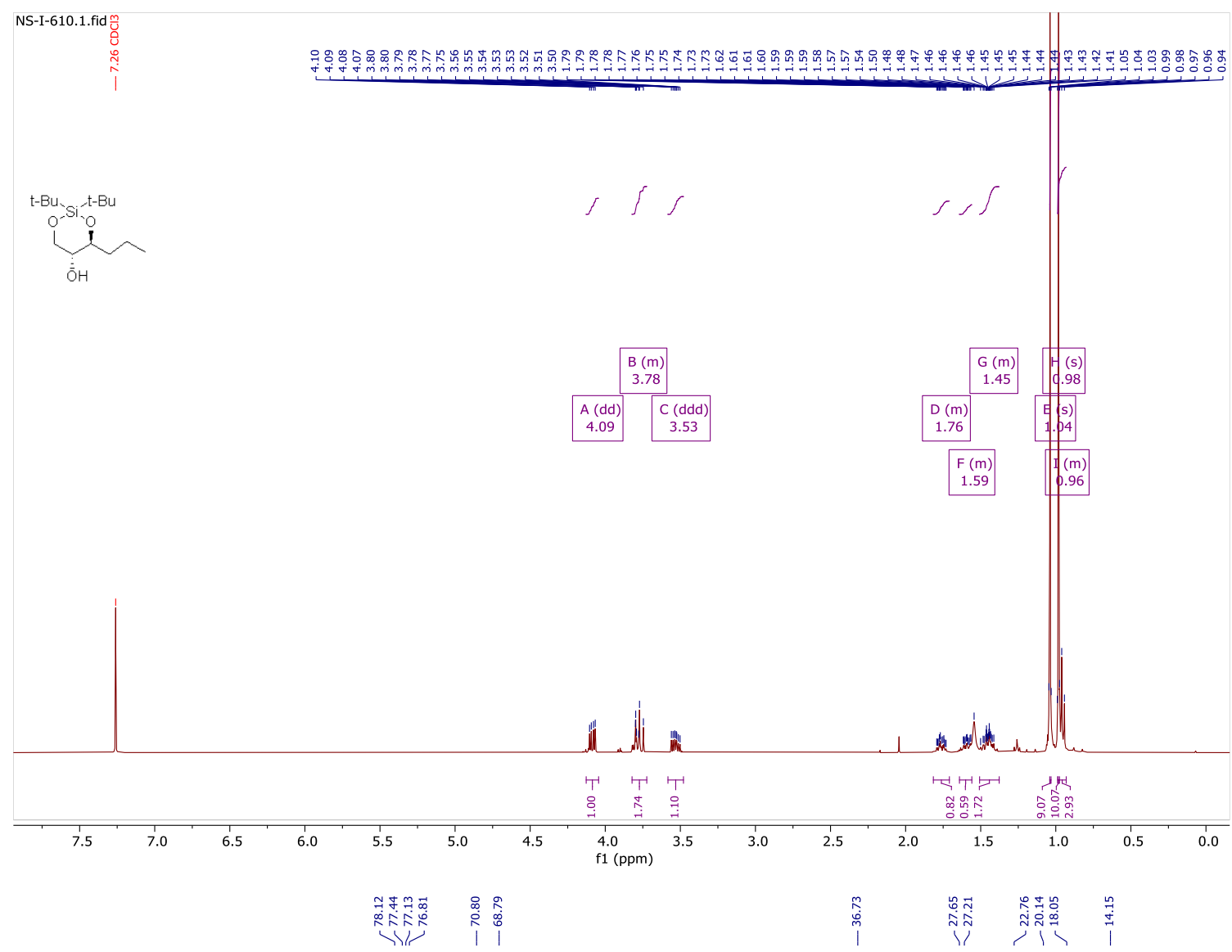

(1)

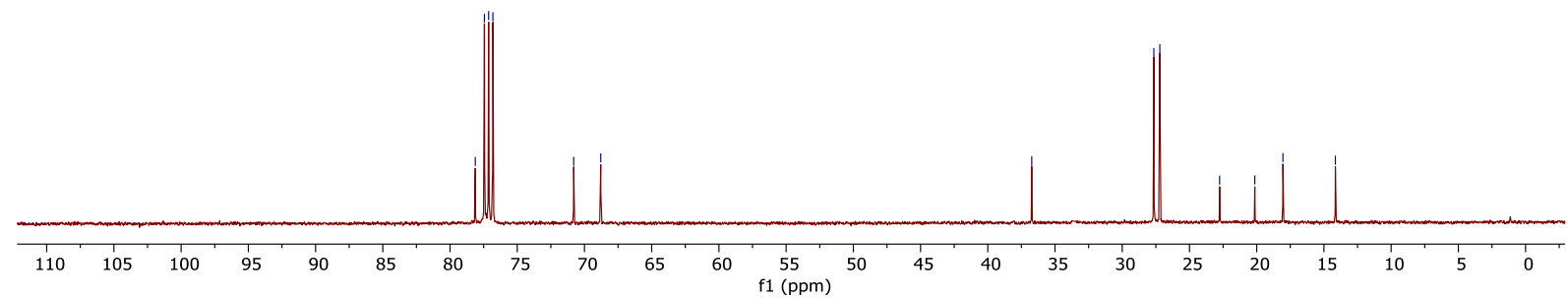



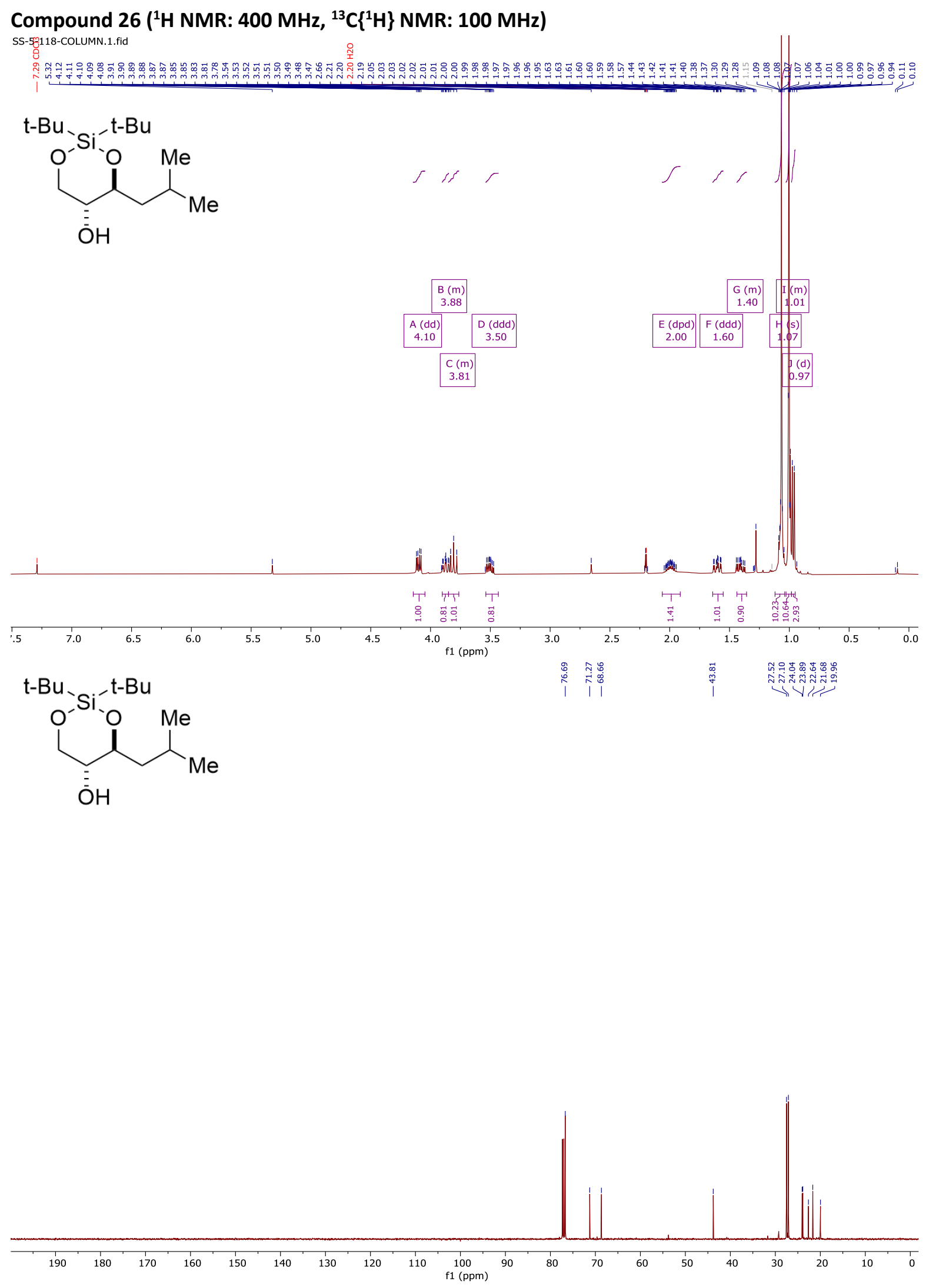
Compound $27\left({ }^{1} \mathrm{H}\right.$ NMR: $400 \mathrm{MHz},{ }^{13} \mathrm{C}\left\{{ }^{1} \mathrm{H}\right\}$ NMR: $100 \mathrm{MHz}$ )
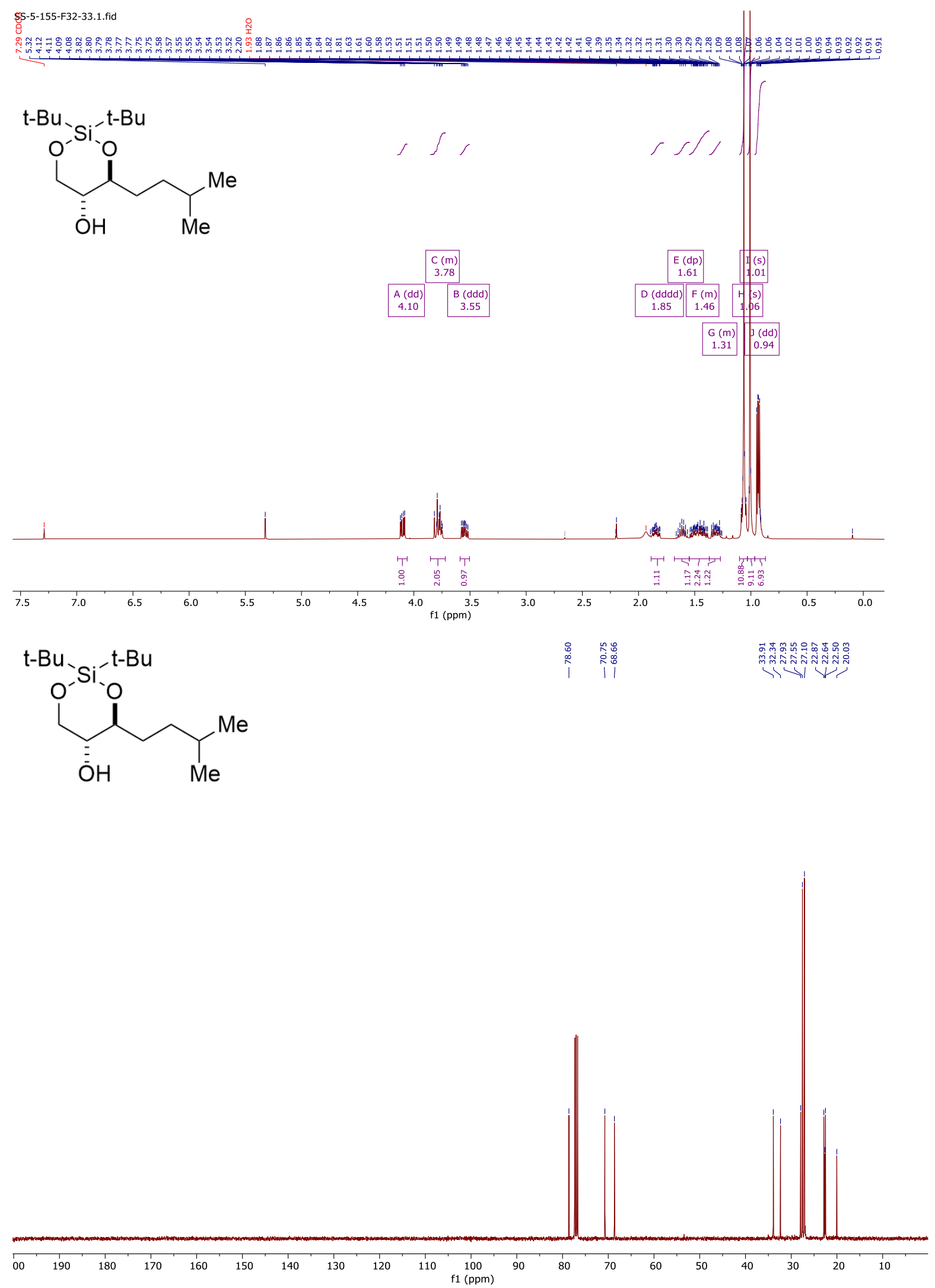
Compound $28\left({ }^{1} \mathrm{H}\right.$ NMR: $400 \mathrm{MHz},{ }^{13} \mathrm{C}\left\{{ }^{1} \mathrm{H}\right\}$ NMR: $\left.100 \mathrm{MHz}\right)$
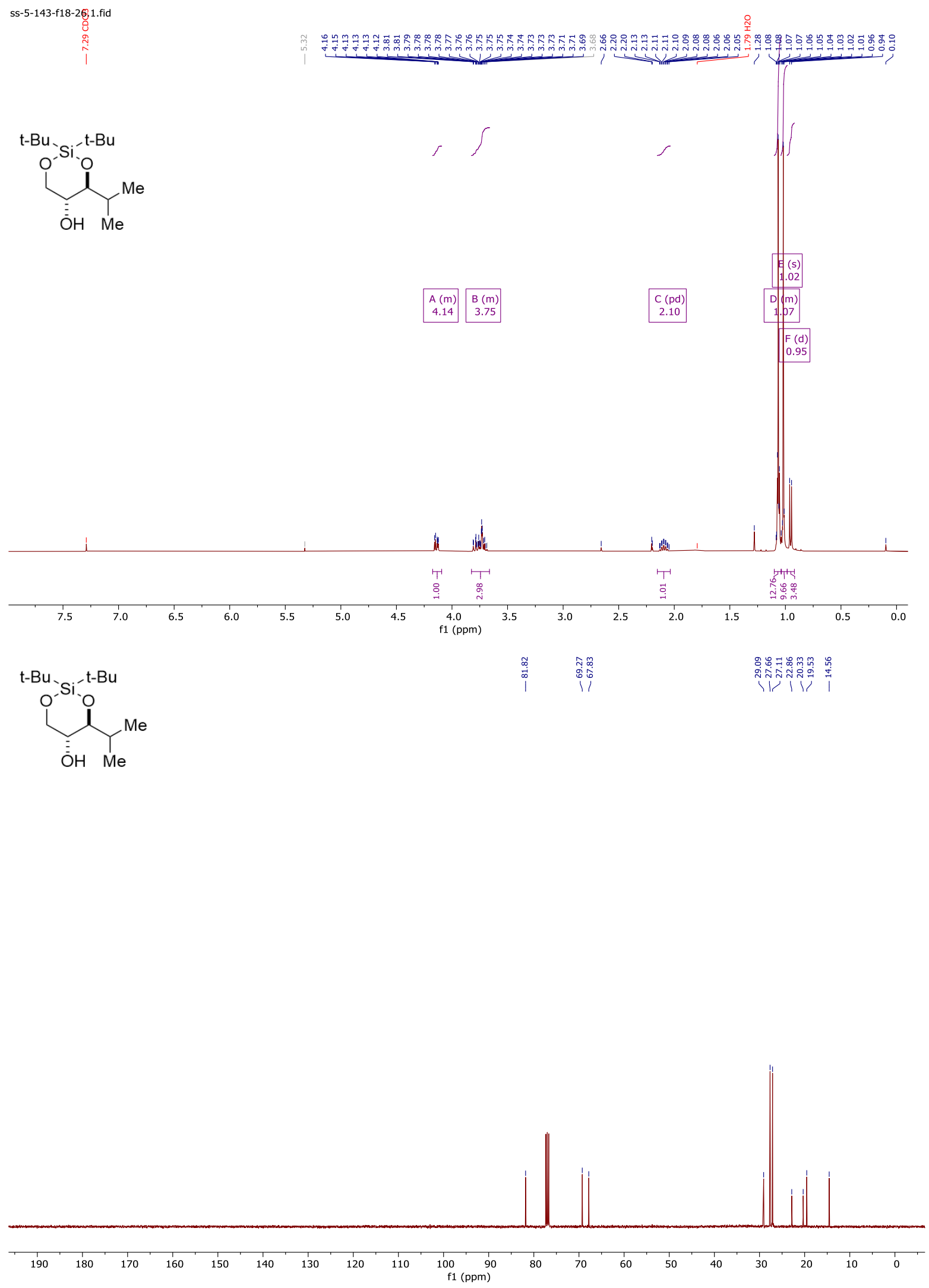
Compound $29\left({ }^{1} \mathrm{H}\right.$ NMR: $400 \mathrm{MHz},{ }^{13} \mathrm{C}\left\{{ }^{1} \mathrm{H}\right\}$ NMR: $\left.100 \mathrm{MHz}\right)$

SS-5-140B-F33.1.fid

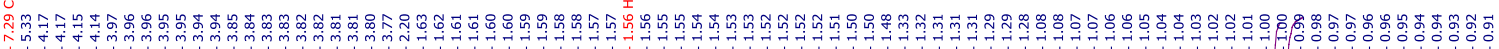

$\underbrace{\mathrm{C}-\mathrm{Bu}}_{\mathrm{Be}}$

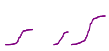
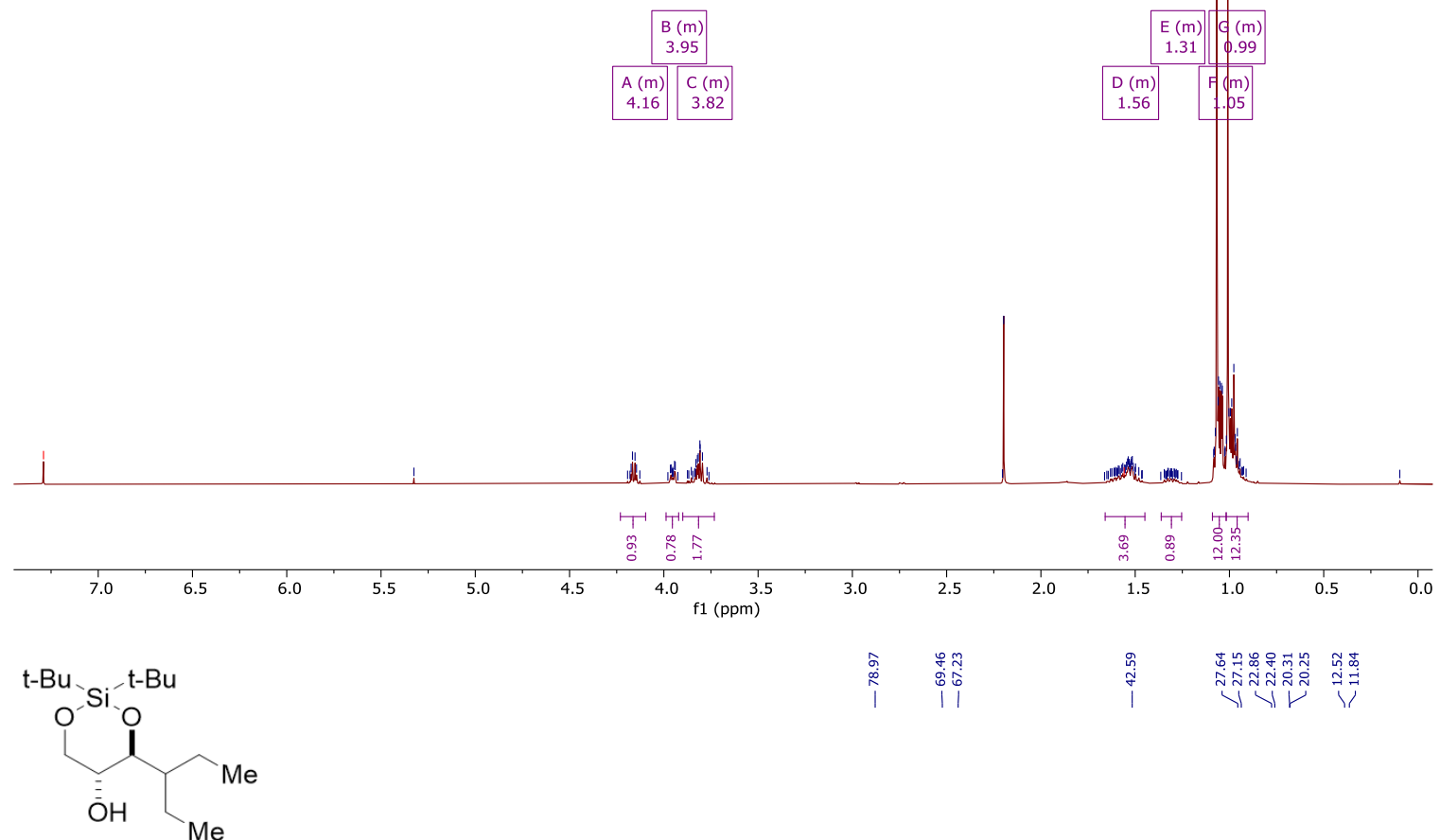

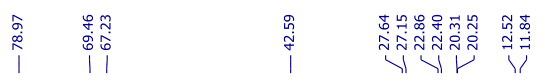

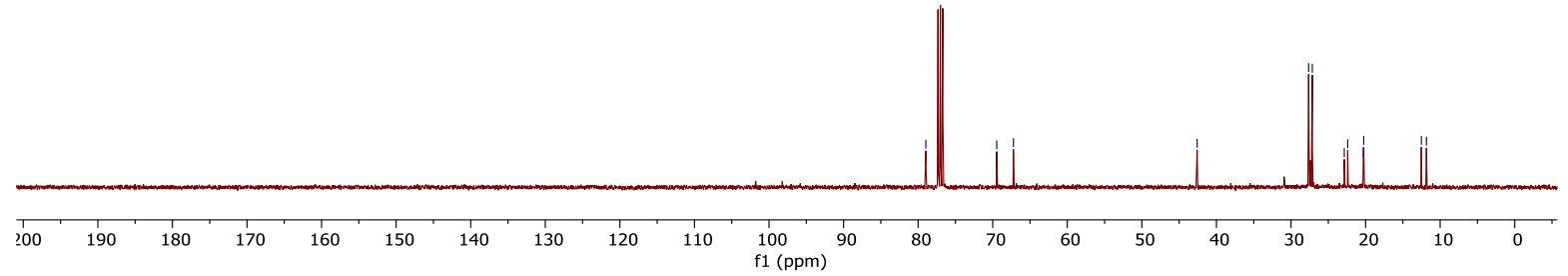




\section{Compound 30 ( ${ }^{1} \mathrm{H}$ NMR: $400 \mathrm{MHz},{ }^{13} \mathrm{C}\left\{{ }^{1} \mathrm{H}\right\}$ NMR: $100 \mathrm{MHz}$ )}

ร్రి-5-115b-f38-42.1. fid

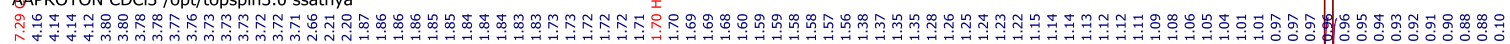
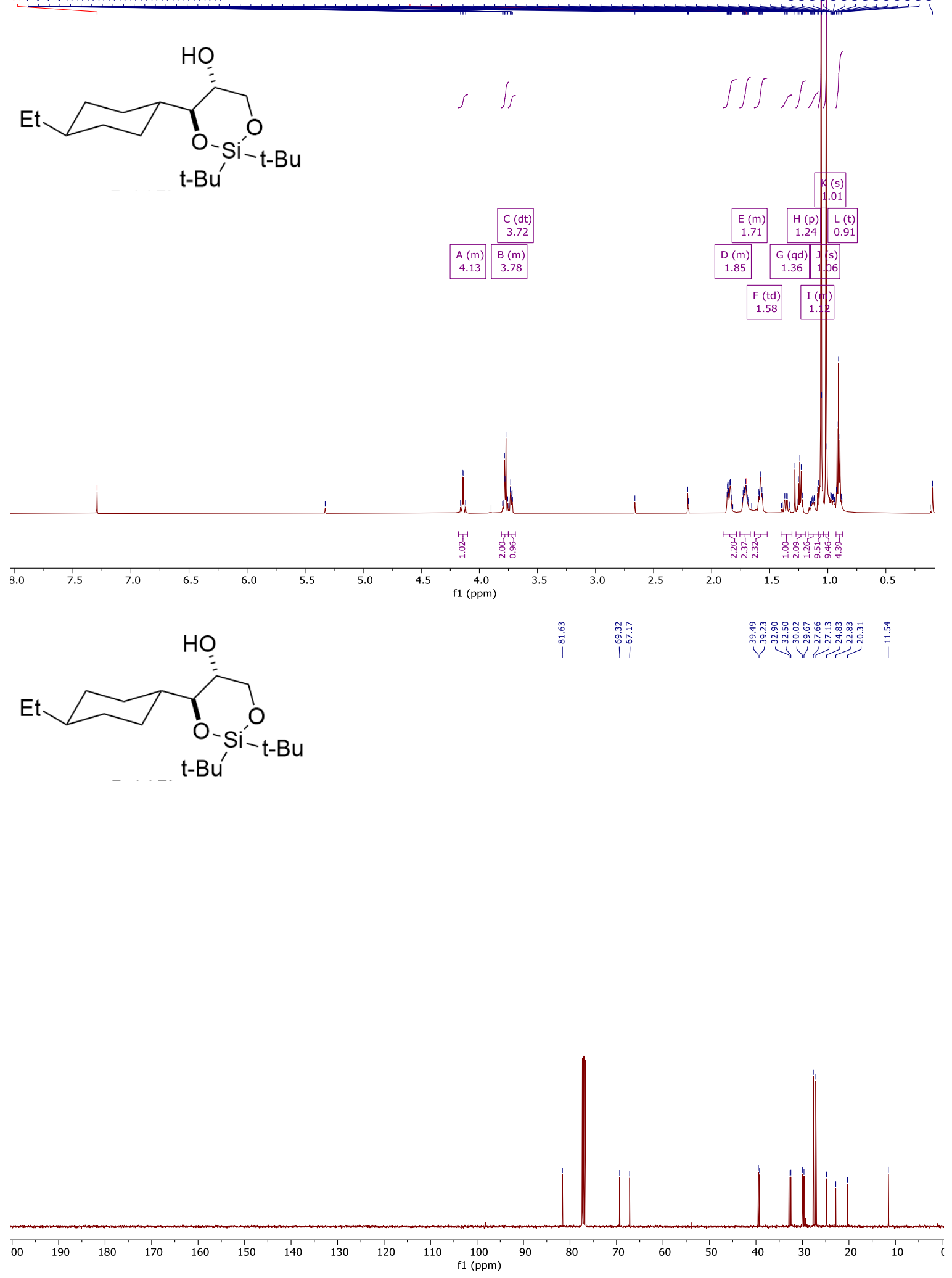
Compound $31\left({ }^{1} \mathrm{H}\right.$ NMR: $400 \mathrm{MHz},{ }^{13} \mathrm{C}\left\{{ }^{1} \mathrm{H}\right\}$ NMR: $\left.125 \mathrm{MHz}\right)$

2ู-111-f57-60.1.fid

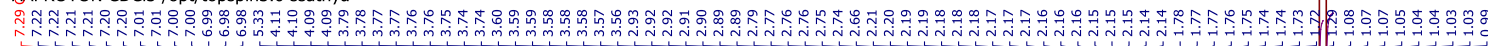
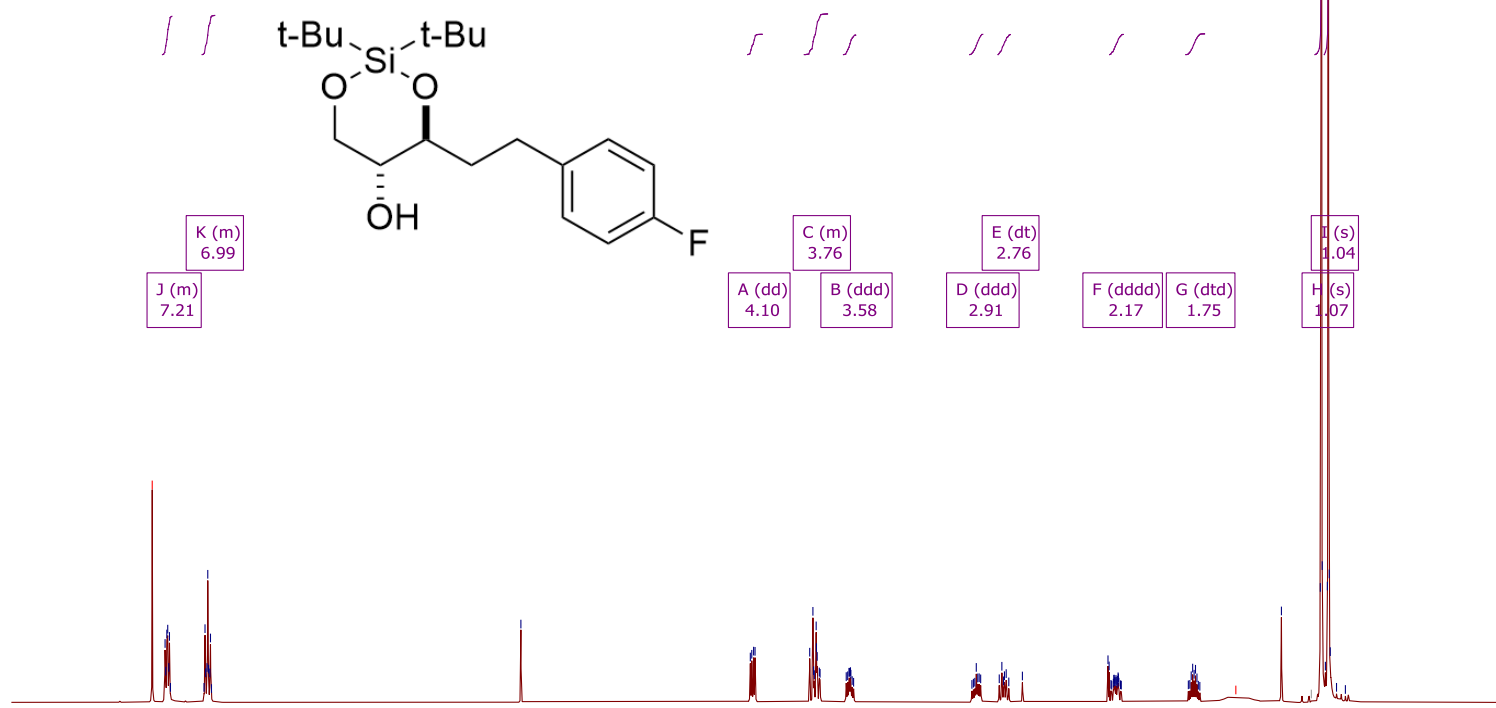

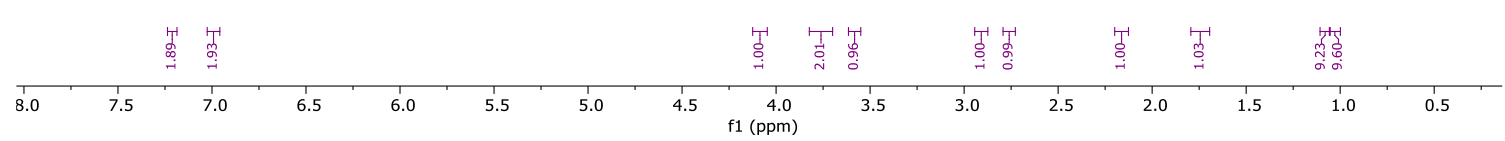

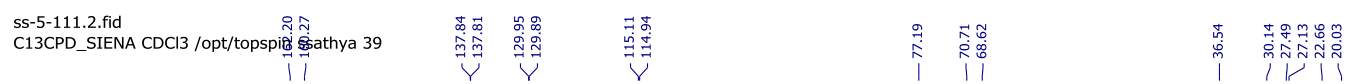

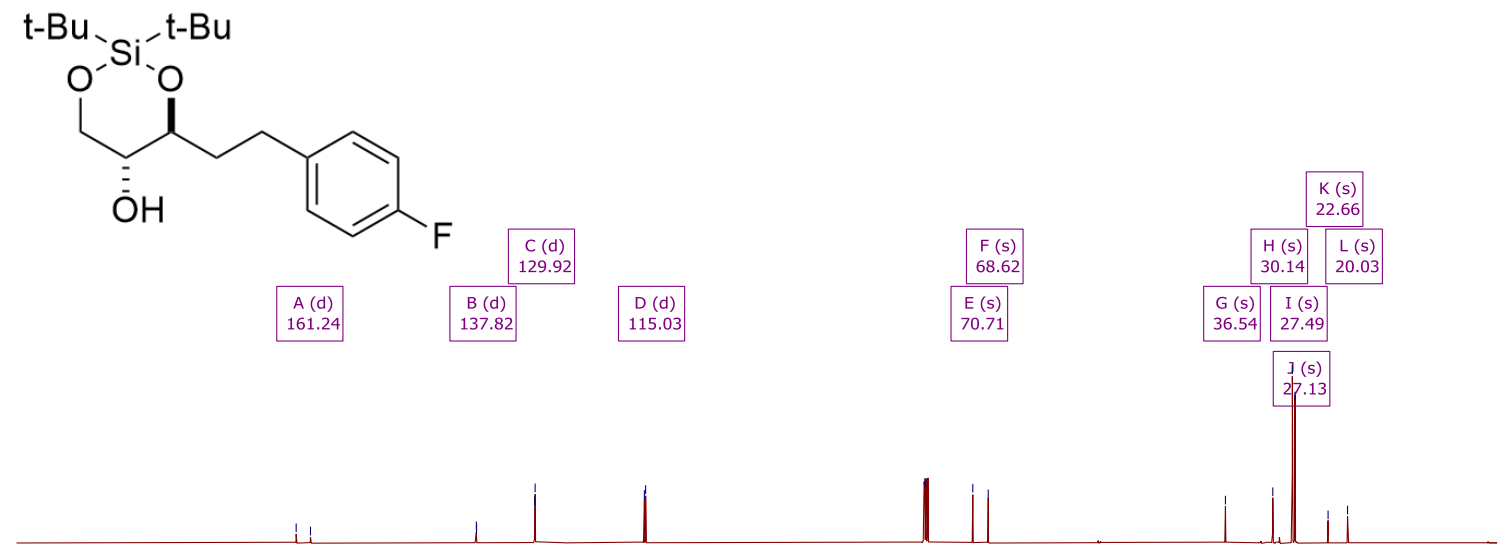

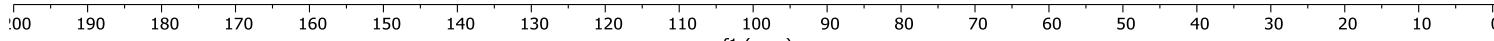


Compound $32\left({ }^{1} \mathrm{H}\right.$ NMR: $400 \mathrm{MHz},{ }^{13} \mathrm{C}\left\{{ }^{1} \mathrm{H}\right\}$ NMR: $\left.100 \mathrm{MHz}\right)$
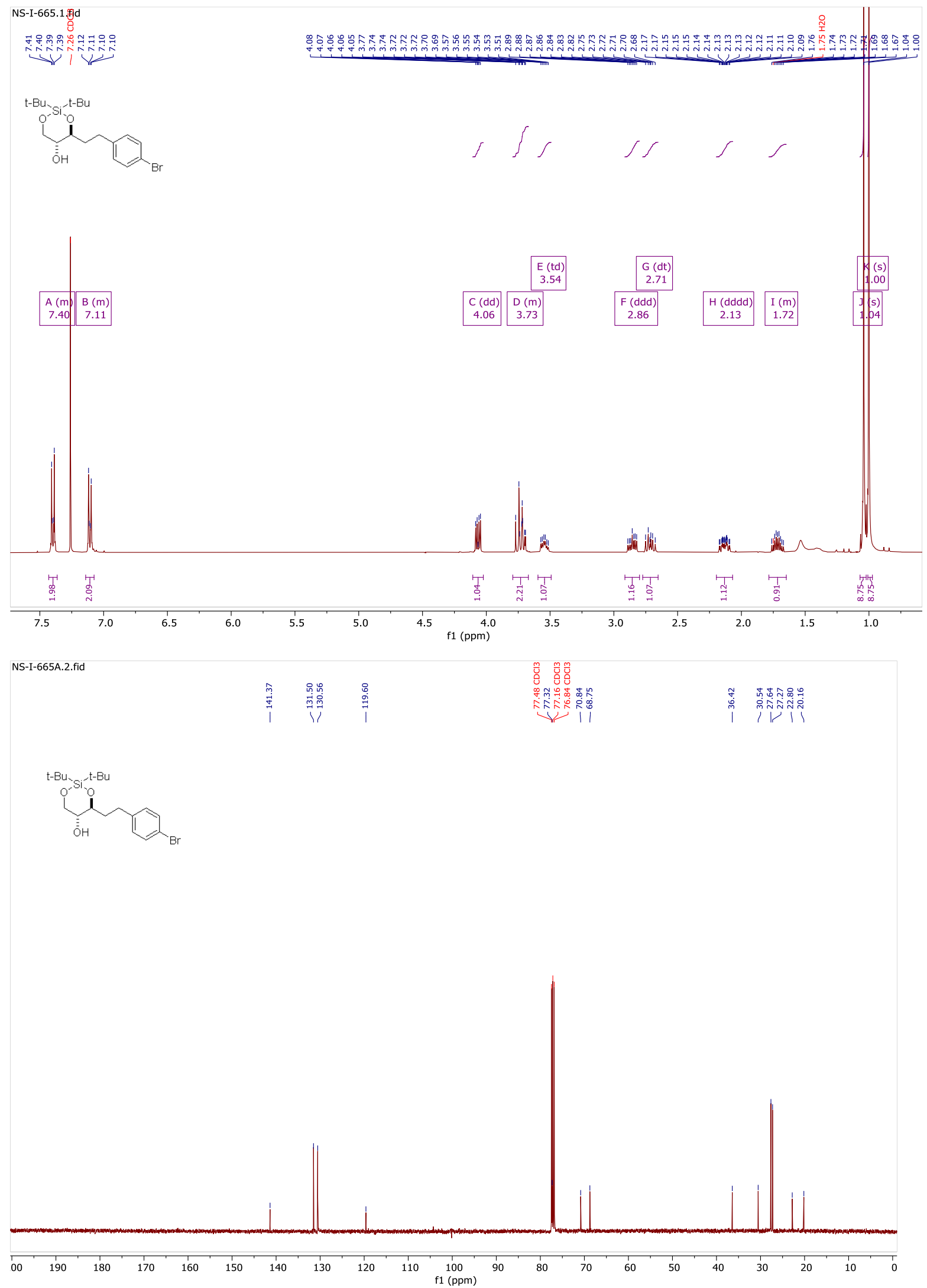
Compound $33\left({ }^{1} \mathrm{H}\right.$ NMR: $400 \mathrm{MHz},{ }^{13} \mathrm{C}\left\{{ }^{1} \mathrm{H}\right\}$ NMR: $\left.125 \mathrm{MHz}\right)$

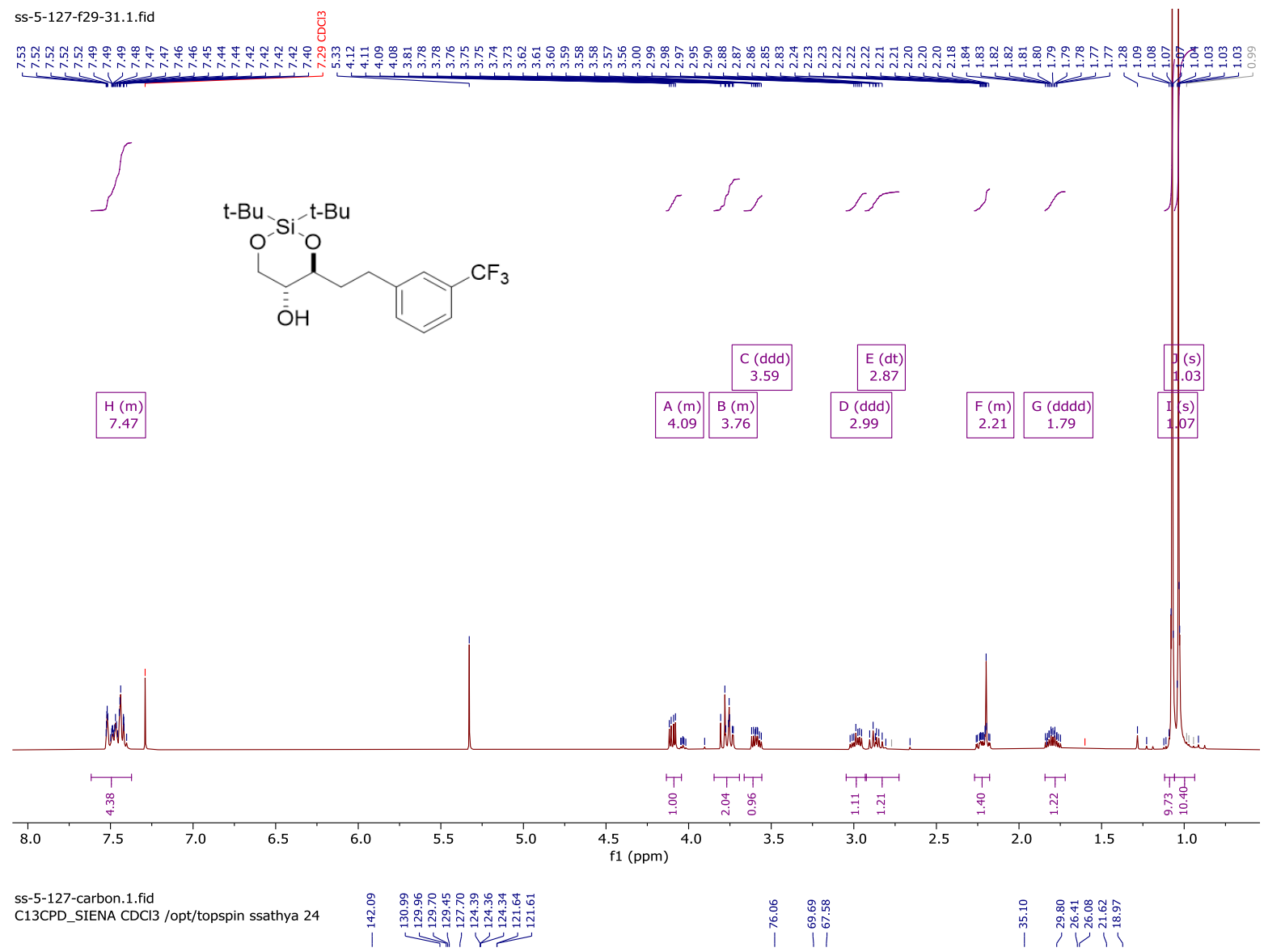<smiles>C[13C](C)(C)[Si][Ge](C)(C)C</smiles>
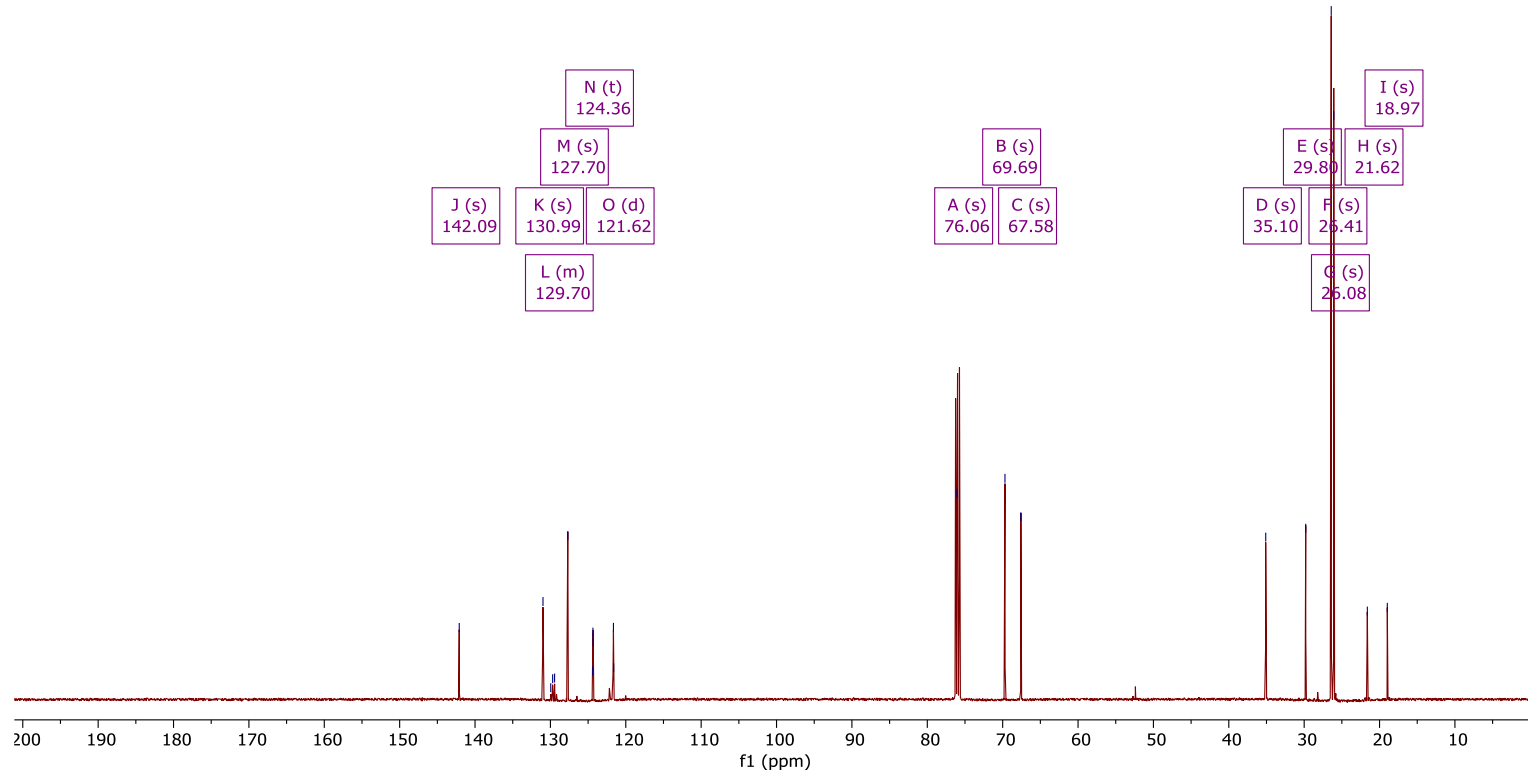
Compound $34\left({ }^{1} \mathrm{H}\right.$ NMR: $400 \mathrm{MHz},{ }^{13} \mathrm{C}\left\{{ }^{1} \mathrm{H}\right\}$ NMR: $\left.100 \mathrm{MHz}\right)$
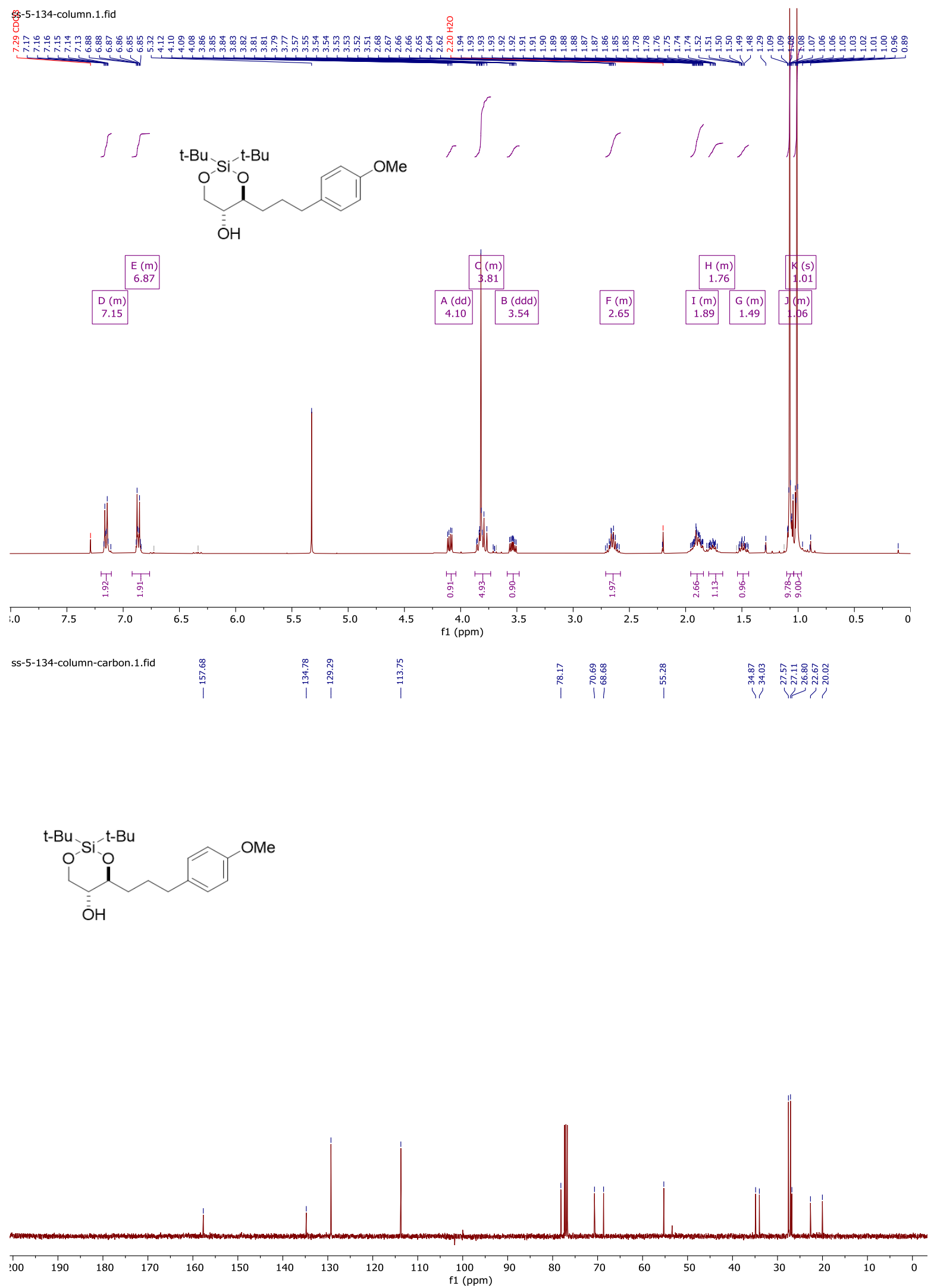
Compound $35\left({ }^{1} \mathrm{H}\right.$ NMR: $400 \mathrm{MHz},{ }^{13} \mathrm{C}\left\{{ }^{1} \mathrm{H}\right\}$ NMR: $\left.100 \mathrm{MHz}\right)$ ss-5-123-column.1.fid

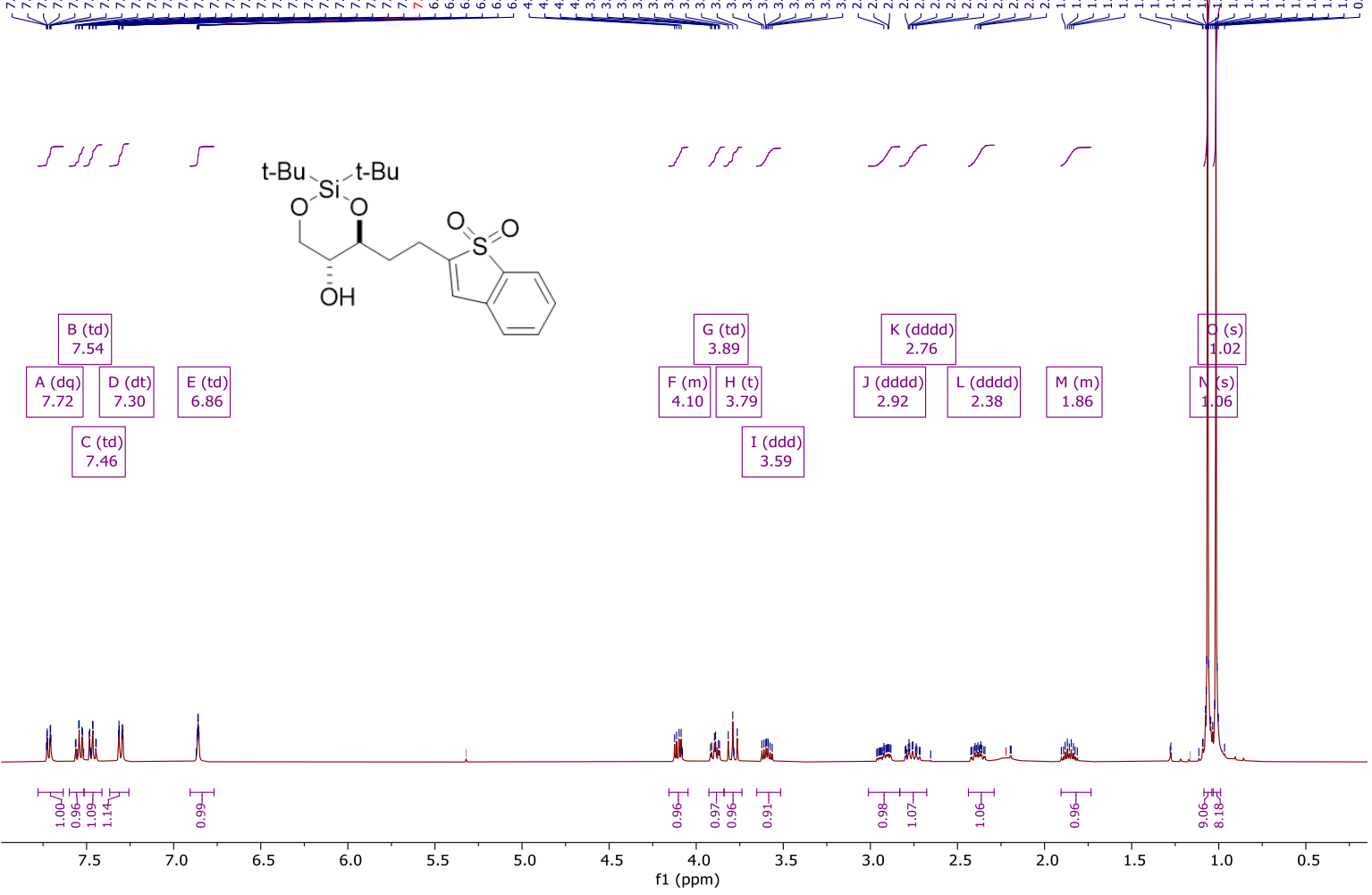

ss-5-123-column-carbon.1.fid
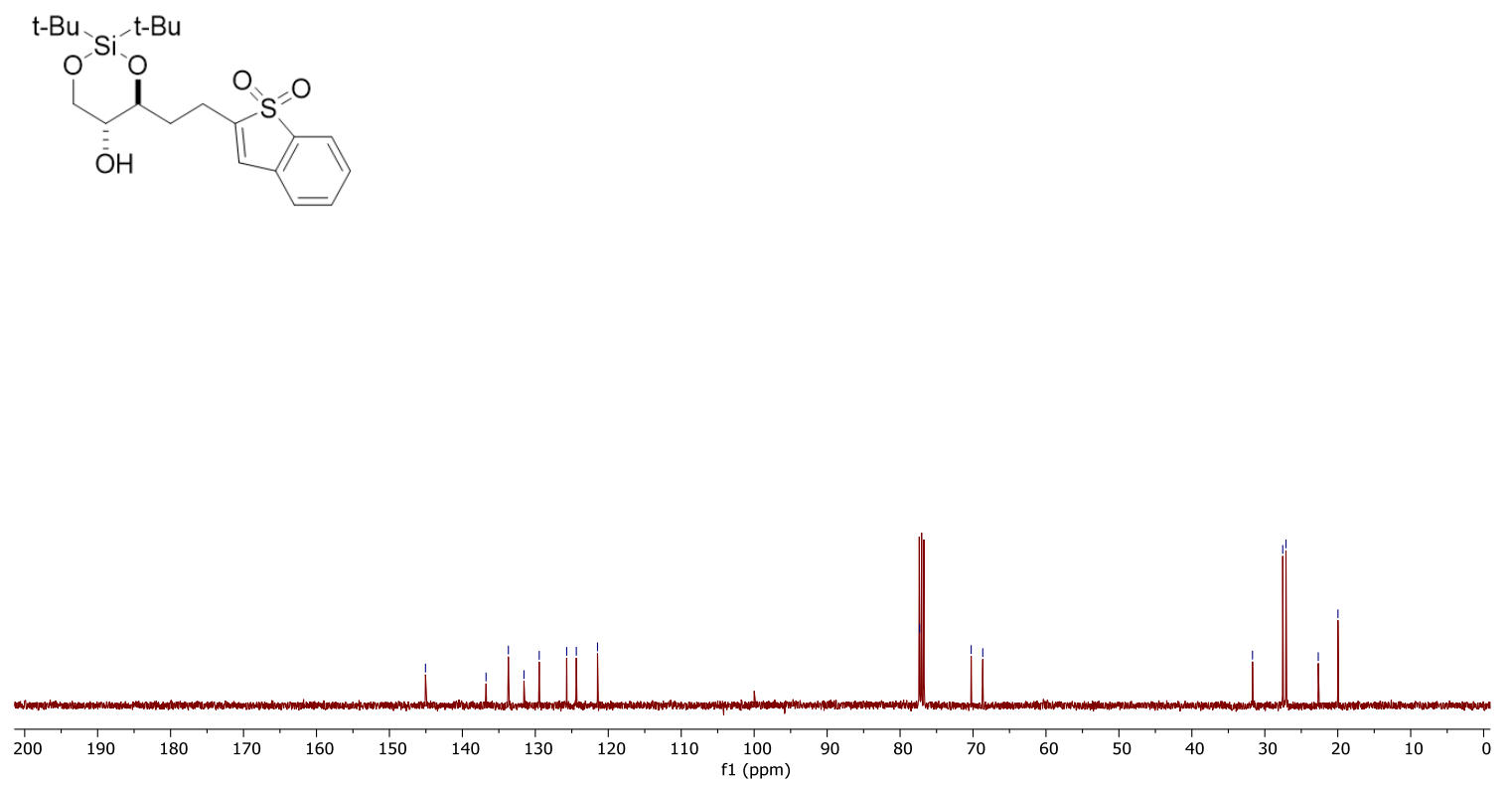
Compound 36 ( ${ }^{1} \mathrm{H}$ NMR: $400 \mathrm{MHz},{ }^{13} \mathrm{C}\left\{{ }^{1} \mathrm{H}\right\}$ NMR: $100 \mathrm{MHz}$ )
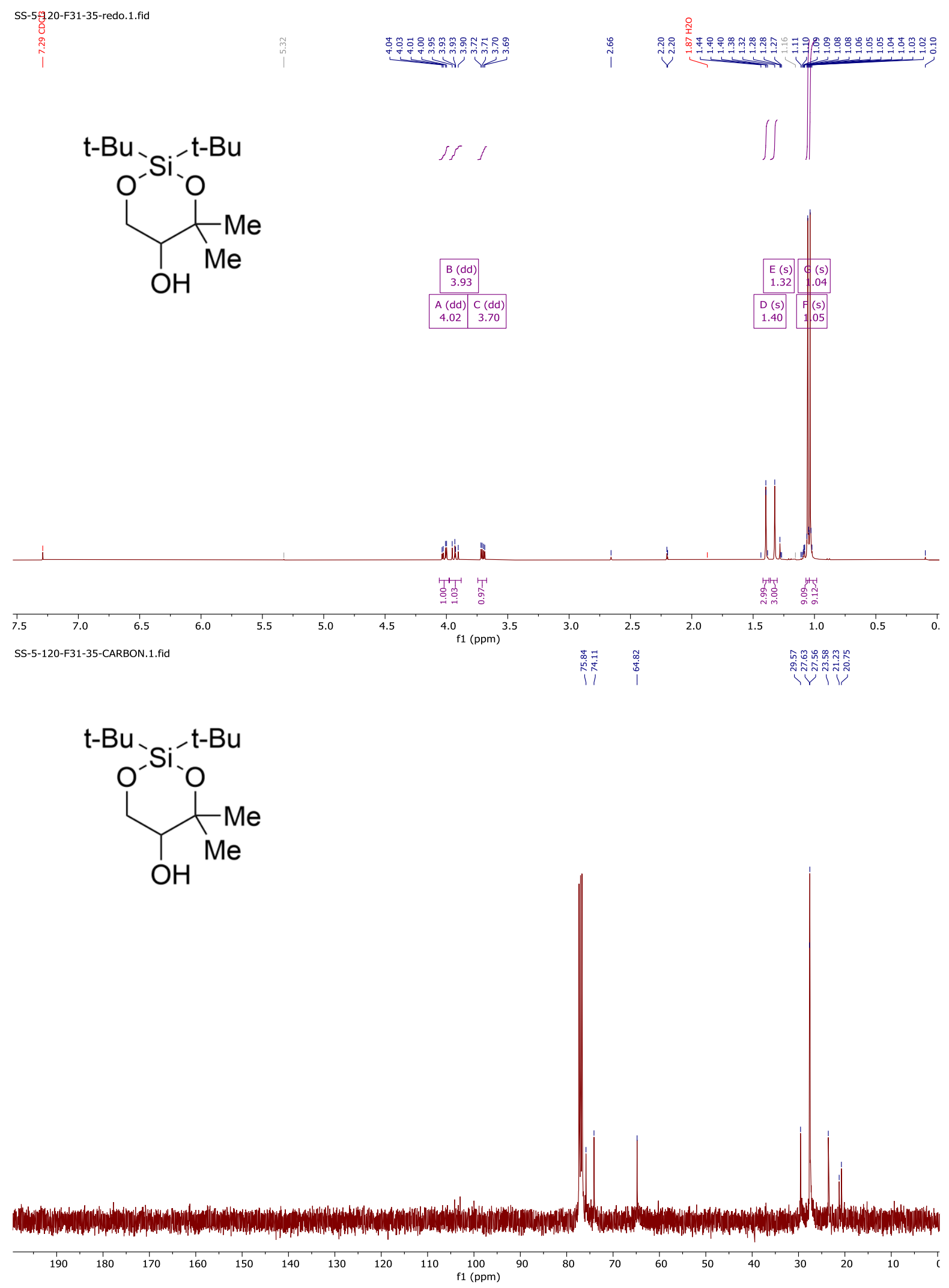
Compound $37\left({ }^{1} \mathrm{H}\right.$ NMR: $400 \mathrm{MHz},{ }^{13} \mathrm{C}\left\{{ }^{1} \mathrm{H}\right\}$ NMR: $100 \mathrm{MHz}$ )

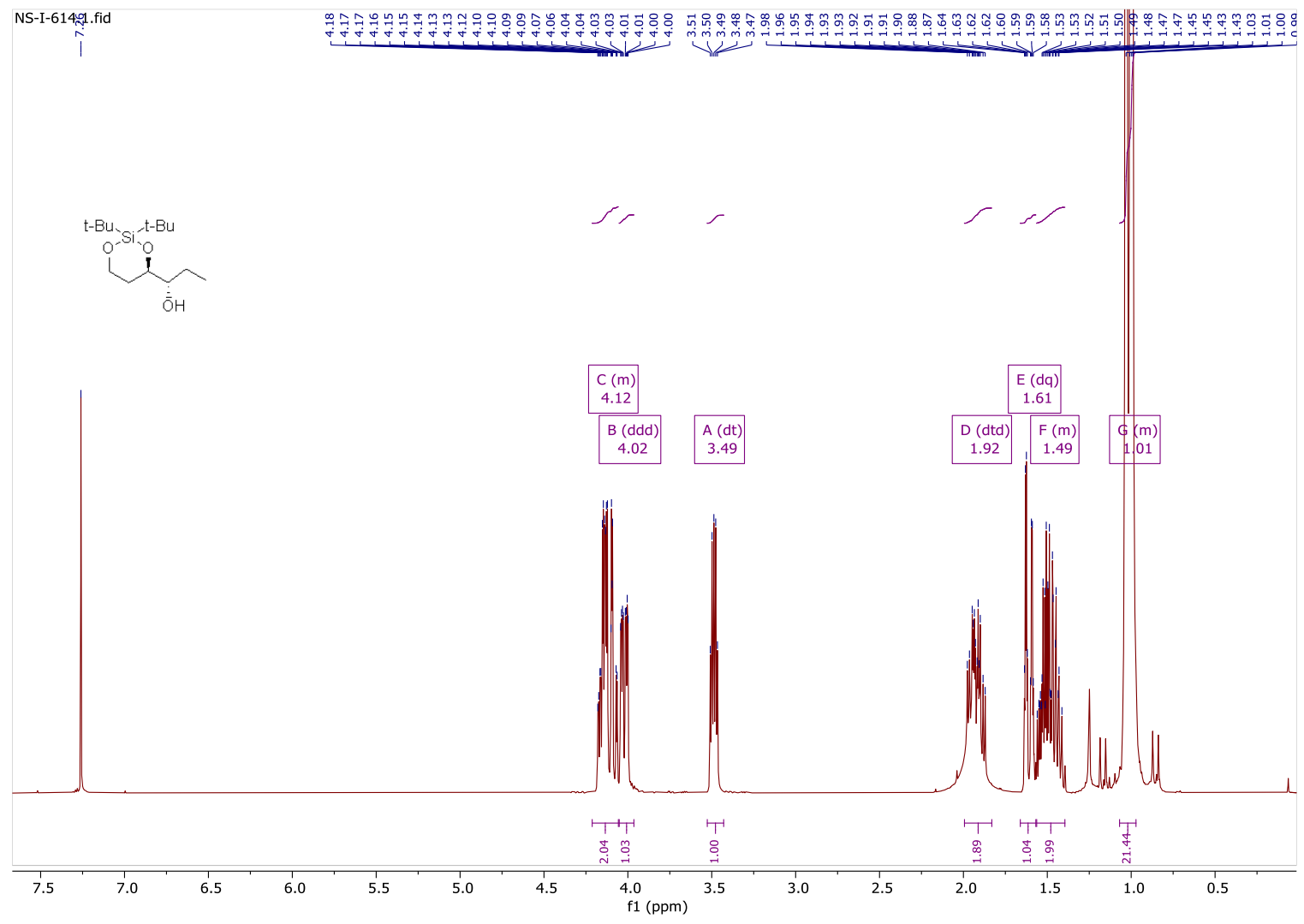

NS-I-614.2.fid
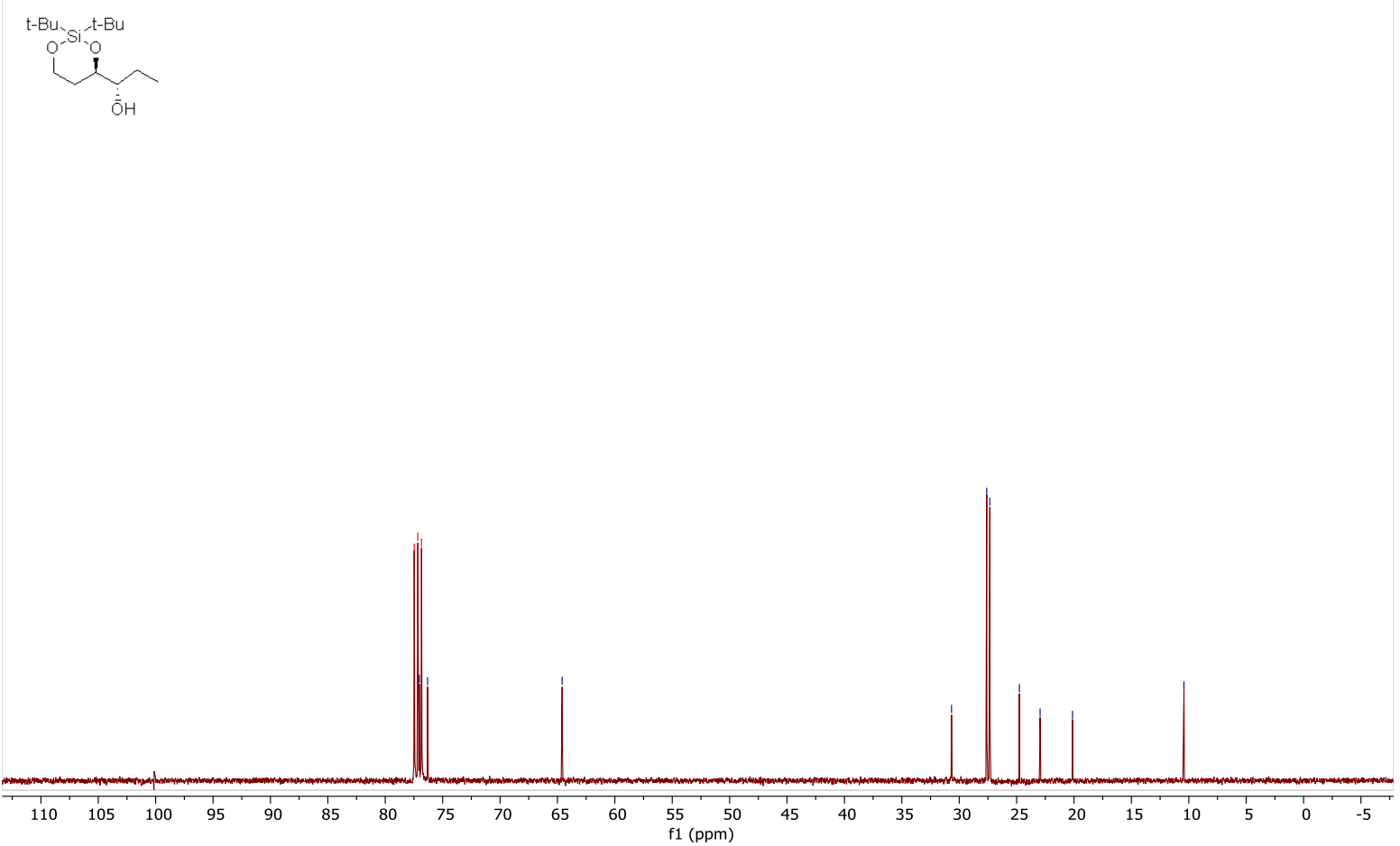
Compound $38\left({ }^{1} \mathrm{H}\right.$ NMR: $400 \mathrm{MHz},{ }^{13} \mathrm{C}\left\{{ }^{1} \mathrm{H}\right\}$ NMR: $\left.100 \mathrm{MHz}\right)$
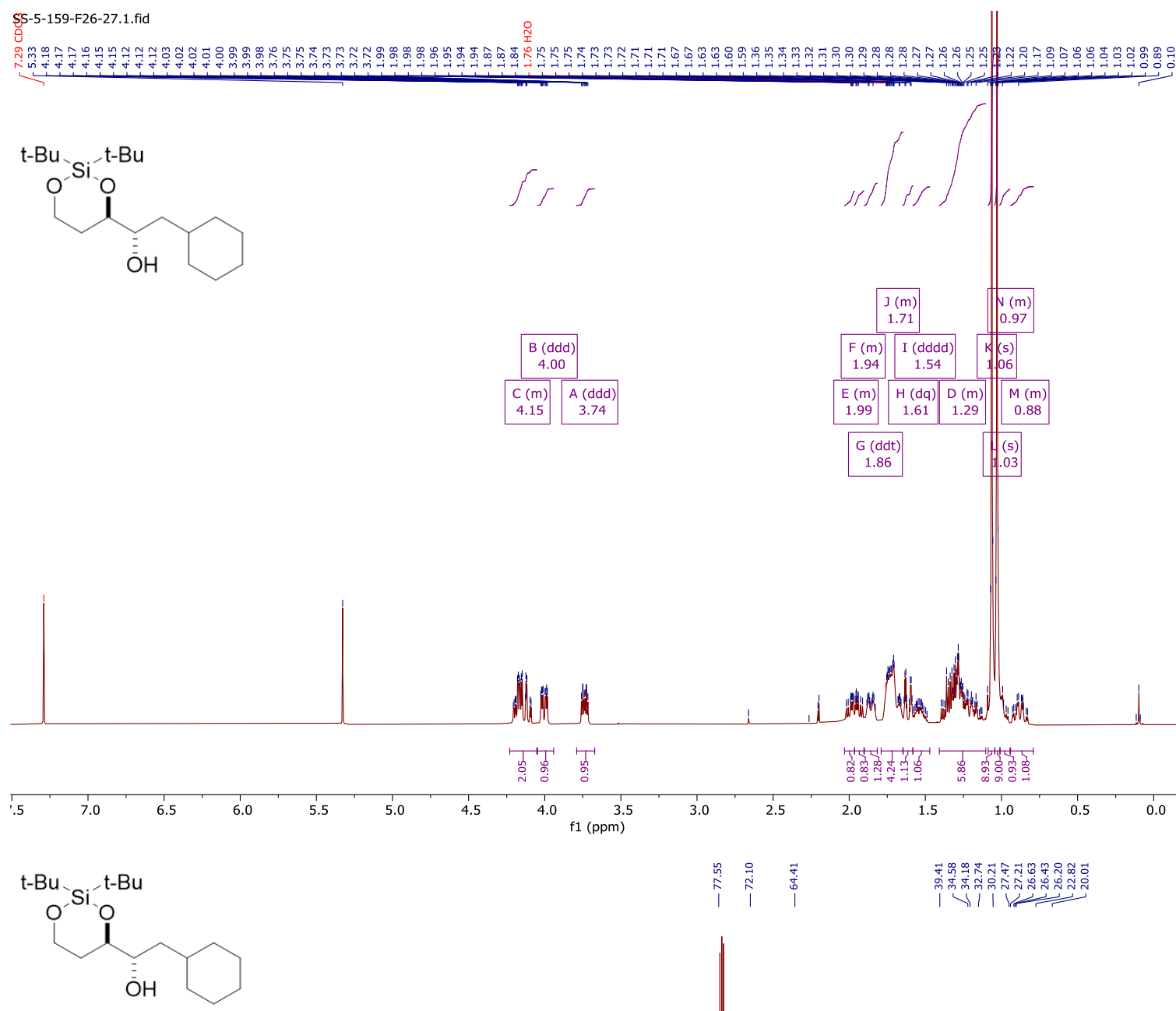

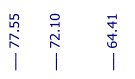

F⿻日禸

(1)

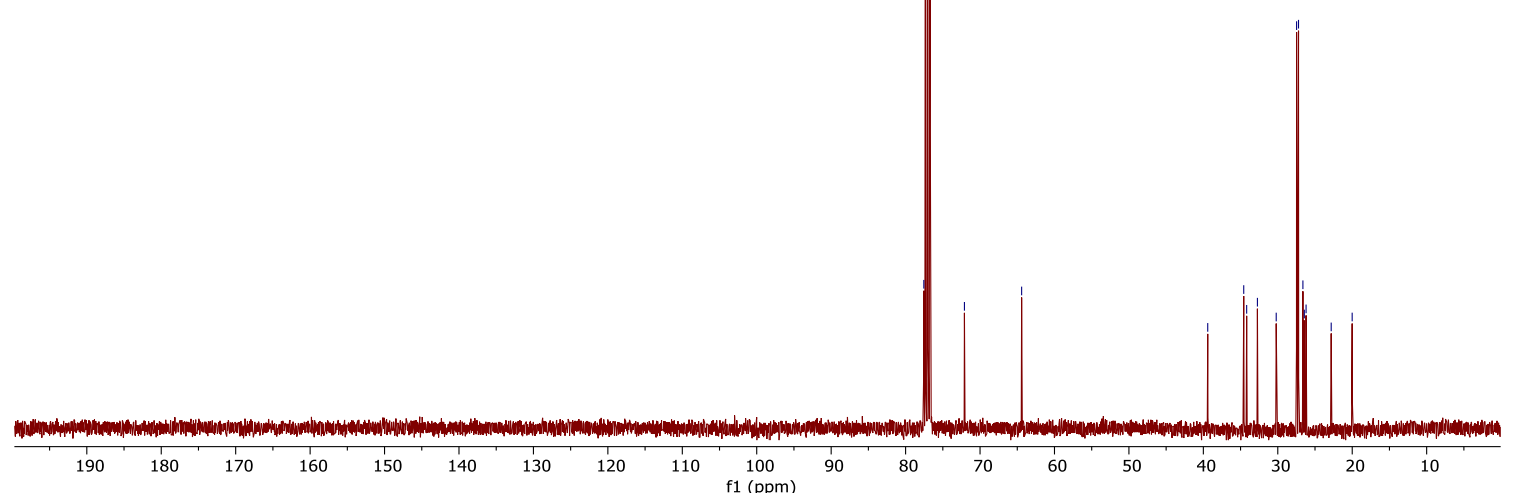


Compound 39 ( ${ }^{1} \mathrm{H}$ NMR: $400 \mathrm{MHz},{ }^{13} \mathrm{C}\left\{{ }^{1} \mathrm{H}\right\}$ NMR: $100 \mathrm{MHz}$ )
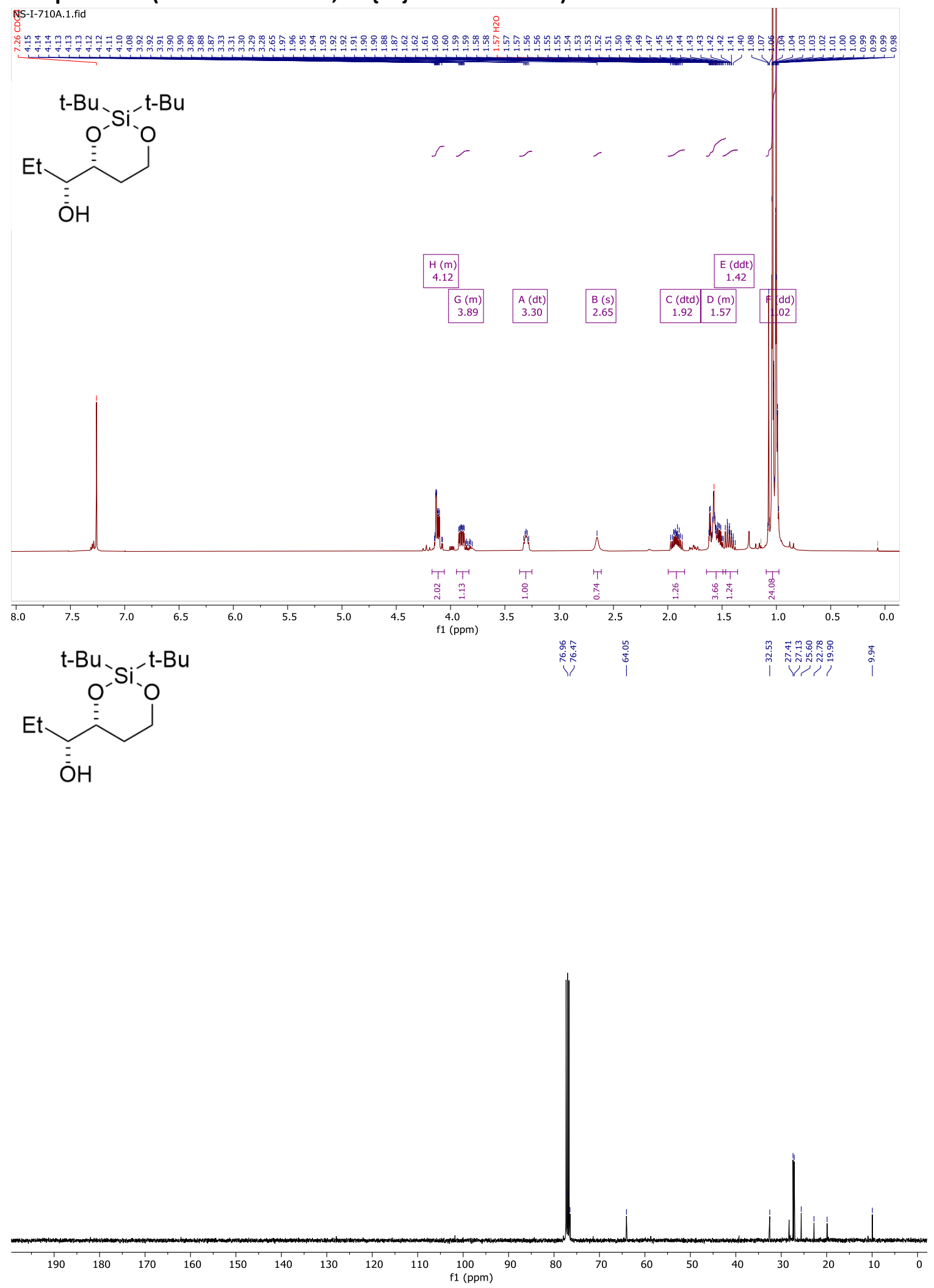
Compound $40\left({ }^{1} \mathrm{H}\right.$ NMR: $400 \mathrm{MHz},{ }^{13} \mathrm{C}\left\{{ }^{1} \mathrm{H}\right\}$ NMR: $\left.100 \mathrm{MHz}\right)$

NS-I 12.1. fid
PROFON CDCl3 /opt/topspin snaga 53

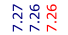

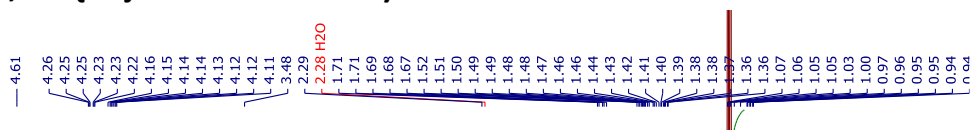

$\overbrace{\mathrm{OH}}^{\mathrm{t}-\mathrm{Bu}}$
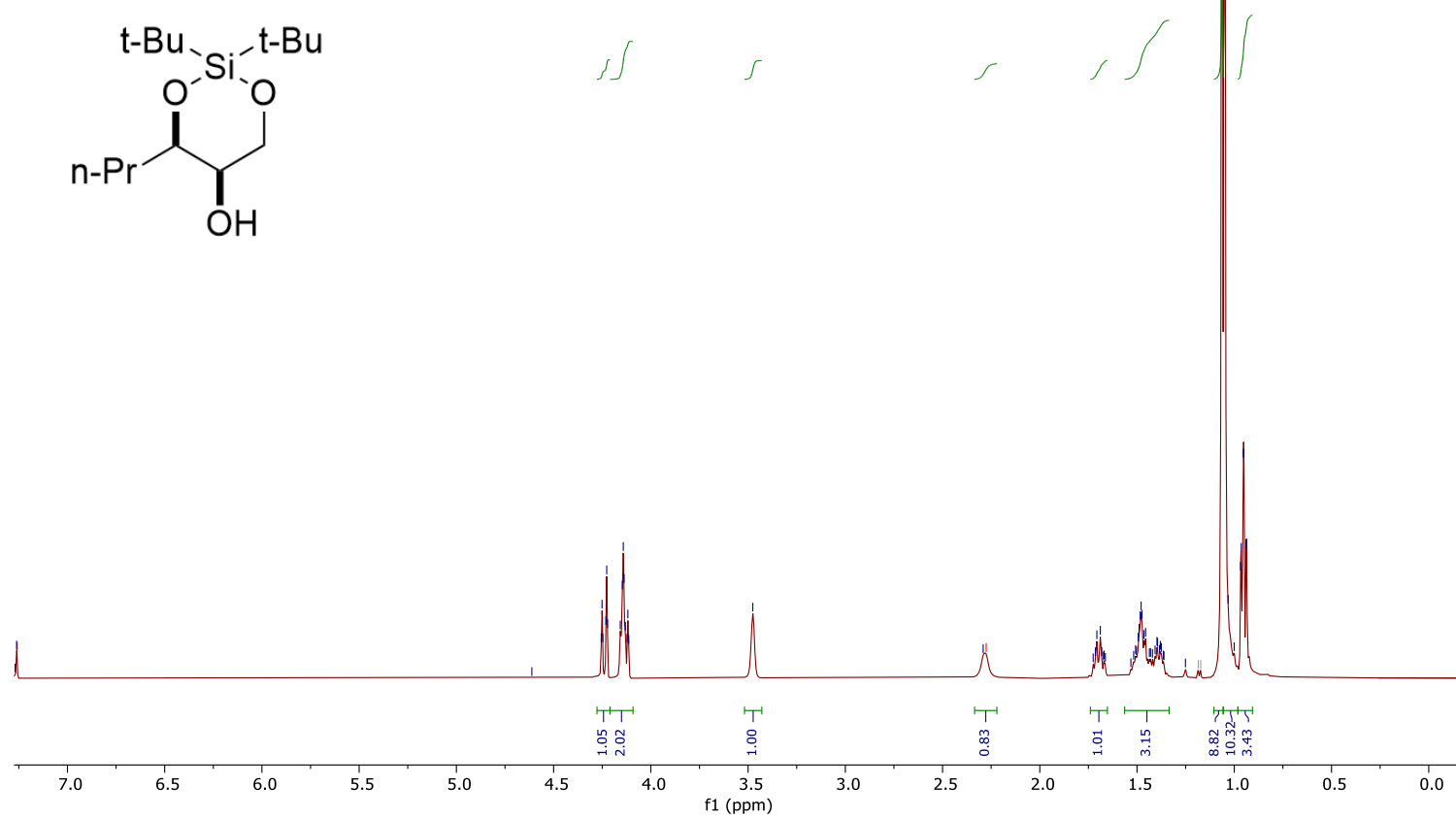

NS-I-712.2.fid

C13CPD_SIENA CDCl3 /opt/topspin snaga 53

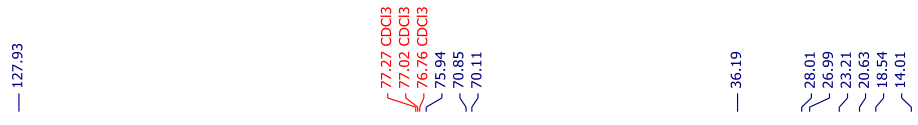

$\overbrace{\mathrm{OH}}^{\mathrm{t}-\mathrm{Bu}}$

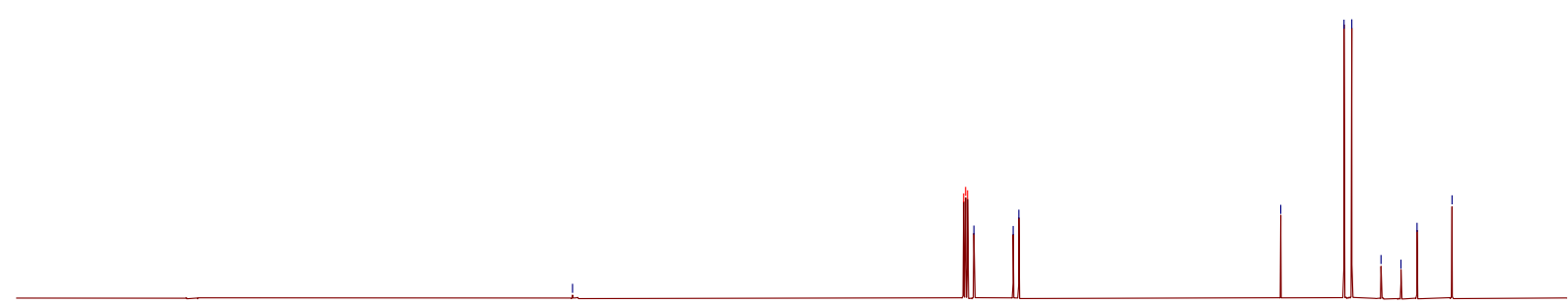

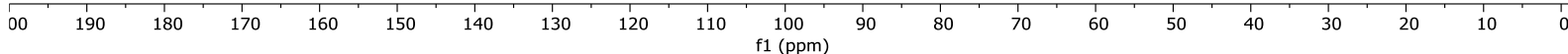


Compound $41\left({ }^{1} \mathrm{H}\right.$ NMR: $400 \mathrm{MHz},{ }^{13} \mathrm{C}\left\{{ }^{1} \mathrm{H}\right\}$ NMR: $\left.100 \mathrm{MHz}\right)$
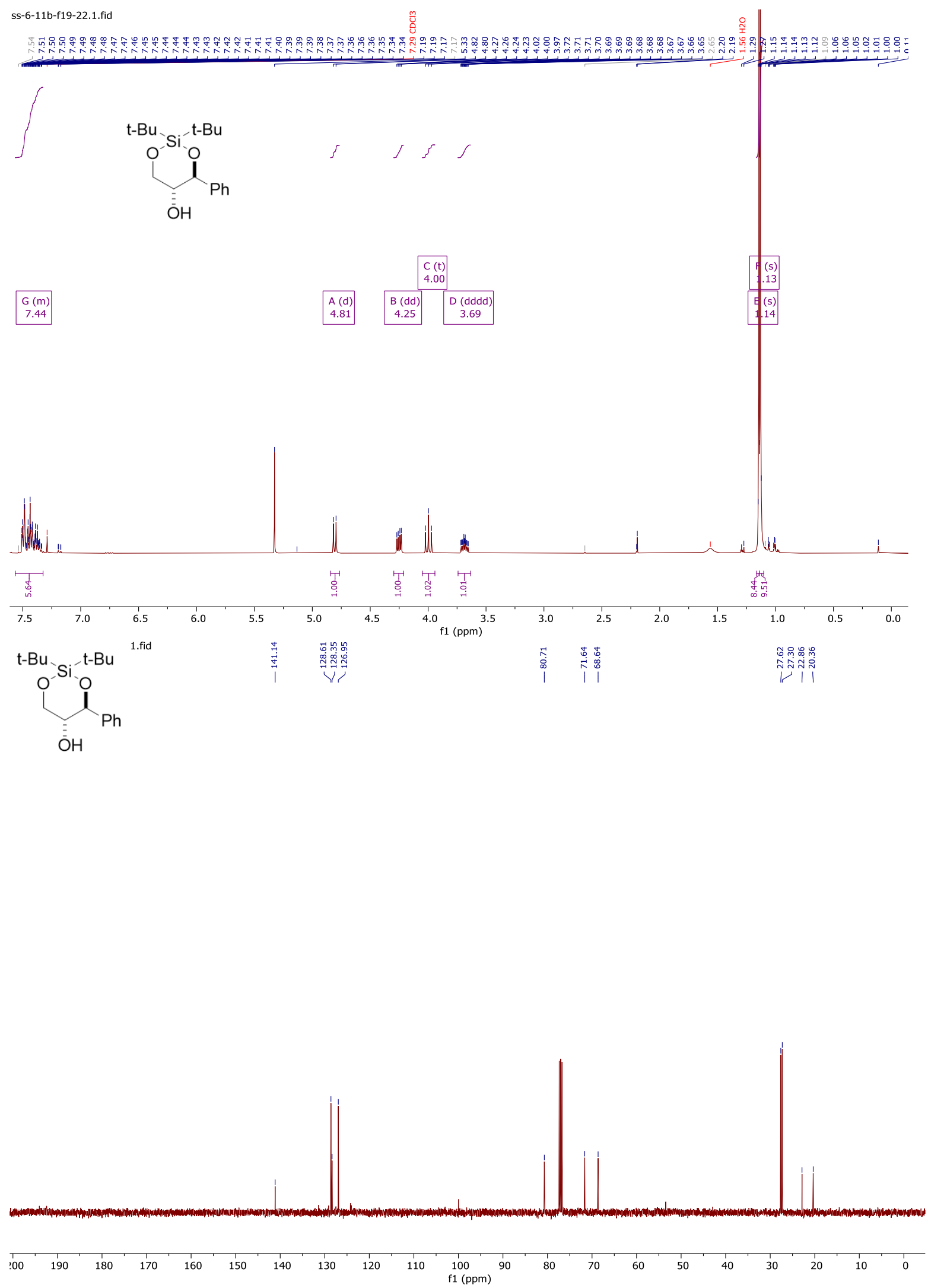
Compound $42\left({ }^{1} \mathrm{H}\right.$ NMR: $400 \mathrm{MHz},{ }^{13} \mathrm{C}\left\{{ }^{1} \mathrm{H}\right\}$ NMR: $\left.100 \mathrm{MHz}\right)$

ss-6-21b-f30-35. हुfid

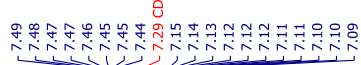

l)

$\int^{\mathrm{t}-\mathrm{Bu}}-\mathrm{Si}_{-}^{-\mathrm{t}-\mathrm{Bu}}$<smiles>O[C@@H]1CO[SiH2]OC1c1ccc(F)cc1</smiles>

$E(m) \quad F(m)$

$7.47 \quad 7.12$

L $2,2 s$

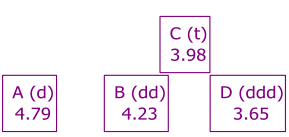

$\begin{array}{ccc}A(d) & B(d d) \\ 4.79 & D\end{array} \quad \begin{gathered}D \\ 3.65\end{gathered}$

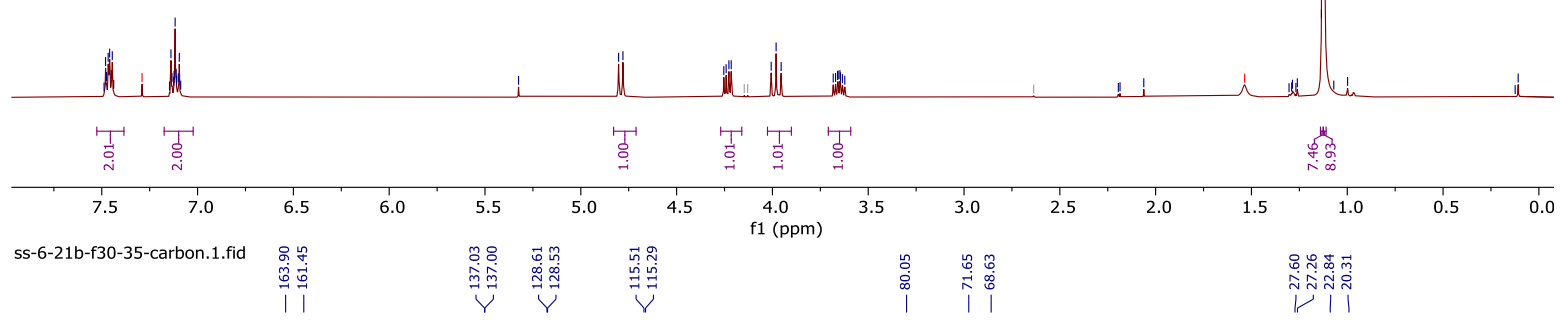<smiles>C[C@H]1CCC([C@H](O)CO[Si](CC(C)(C)C)(C(C)(C)C)C(C)(C)C)OC1</smiles>

\begin{tabular}{|c|c|c|c|}
\hline & & $\begin{array}{c}C(d) \\
128.57\end{array}$ & \\
\hline $\begin{array}{c}A(d) \\
162.67\end{array}$ & $\begin{array}{c}B(d) \\
137.02\end{array}$ & & $\begin{array}{c}D(d) \\
115.40\end{array}$ \\
\hline
\end{tabular}

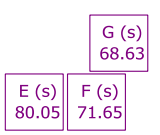

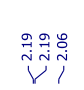

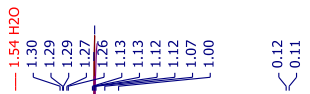


Compound $43\left({ }^{1} \mathrm{H}\right.$ NMR: $400 \mathrm{MHz},{ }^{13} \mathrm{C}\left\{{ }^{1} \mathrm{H}\right\}$ NMR: $\left.100 \mathrm{MHz}\right)$ SS-6-15-CoLUMN.1.fid

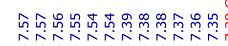

t-Bu, ${ }^{-1-i^{-}-B u}$<smiles>O[C@H]1[Ge]OC[C@H](O)[C@H]1c1ccc(Br)cc1</smiles>
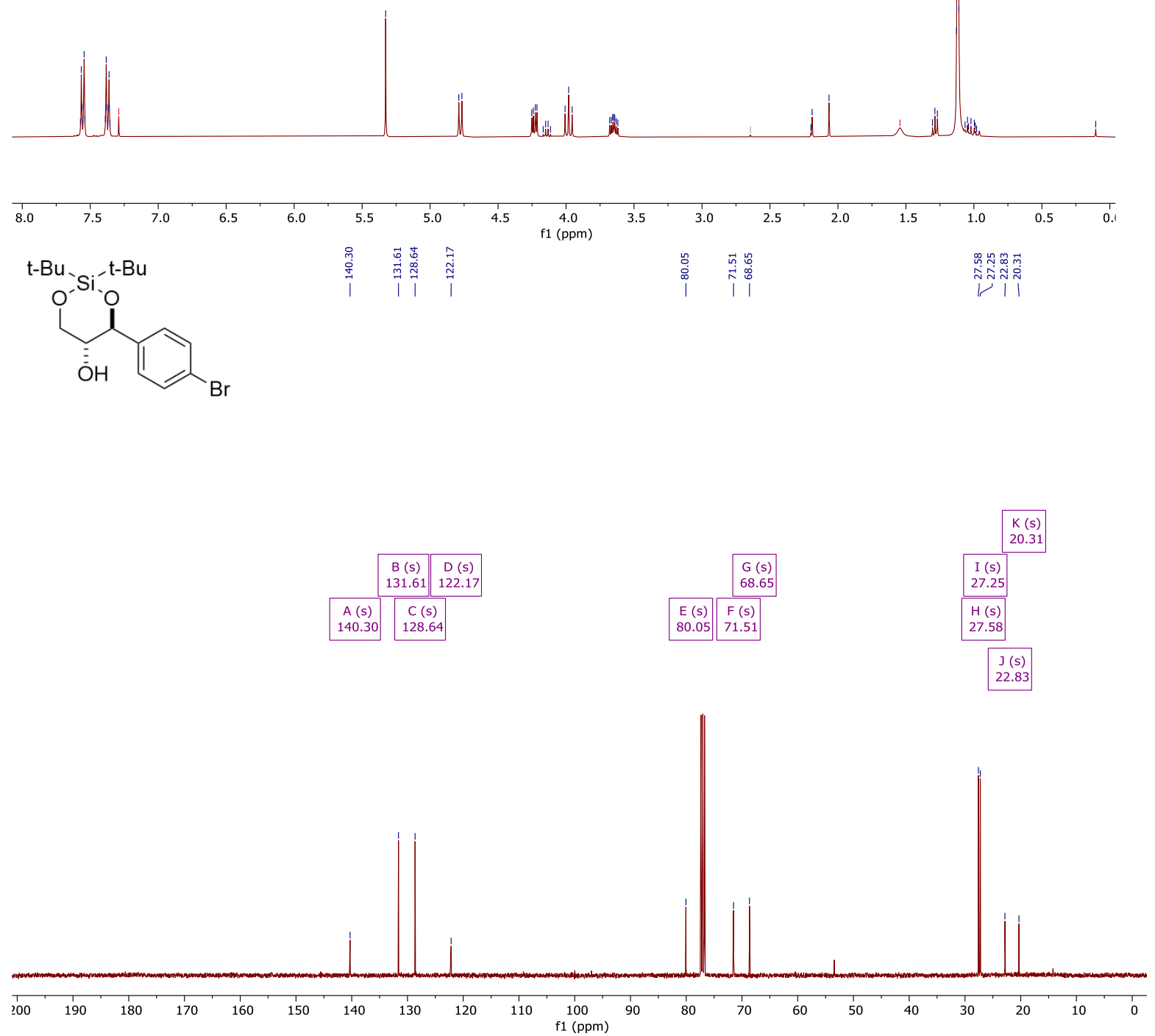
Compound $44\left({ }^{1} \mathrm{H}\right.$ NMR: $400 \mathrm{MHz},{ }^{13} \mathrm{C}\left\{{ }^{1} \mathrm{H}\right\}$ NMR: $\left.100 \mathrm{MHz}\right)$ Ss-6-28-28-33.1.fid

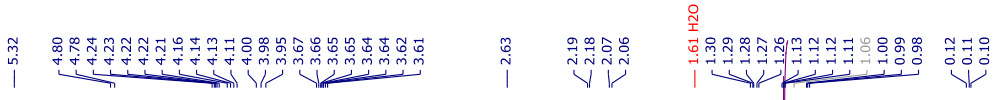
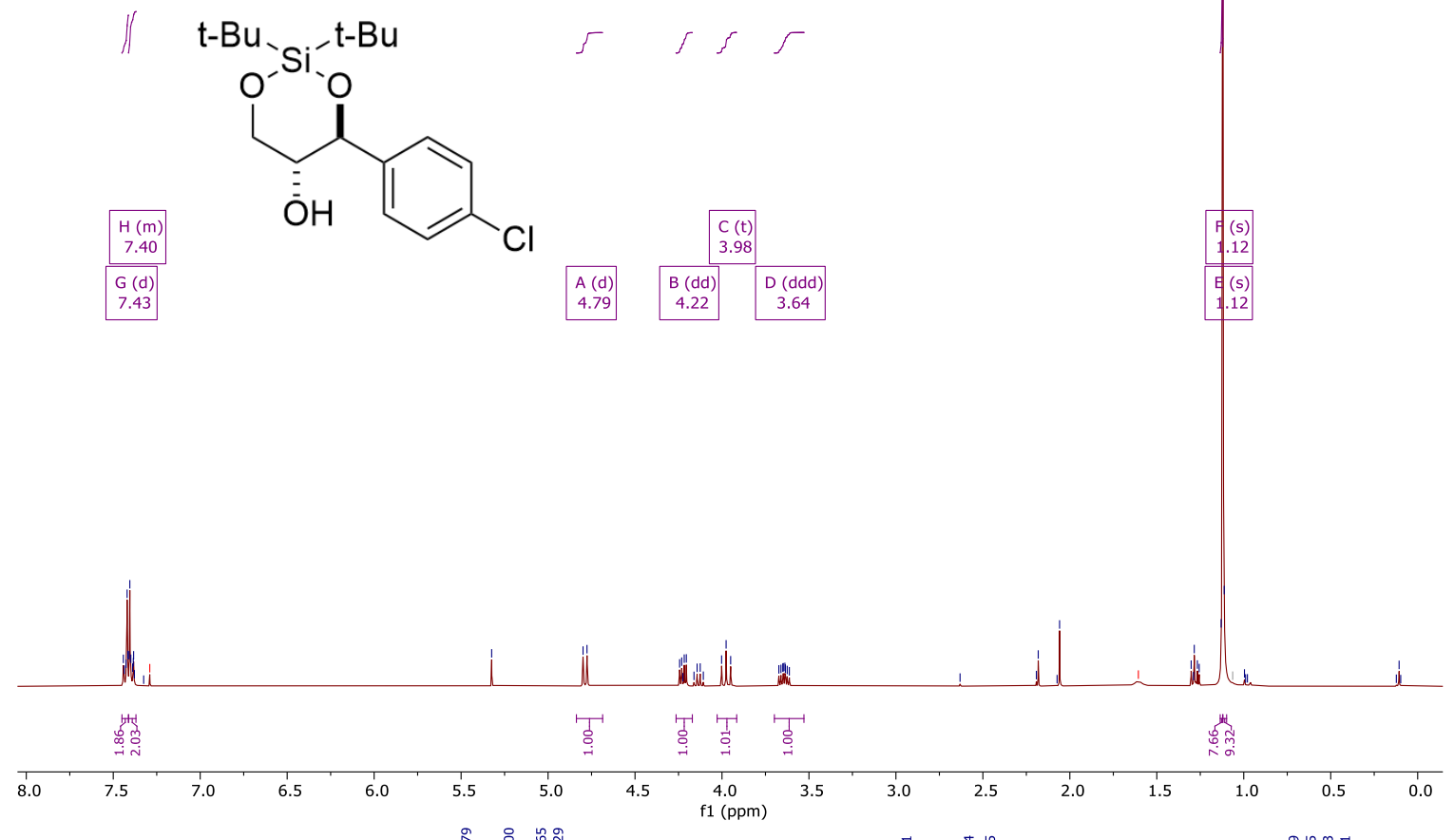<smiles>CC(C)(C)[Si]1(C(C)(C)C)OC[C@@H](O)C(c2ccc(Cl)cc2)O1</smiles>

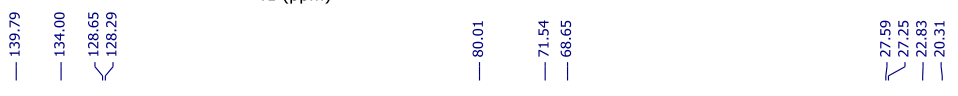

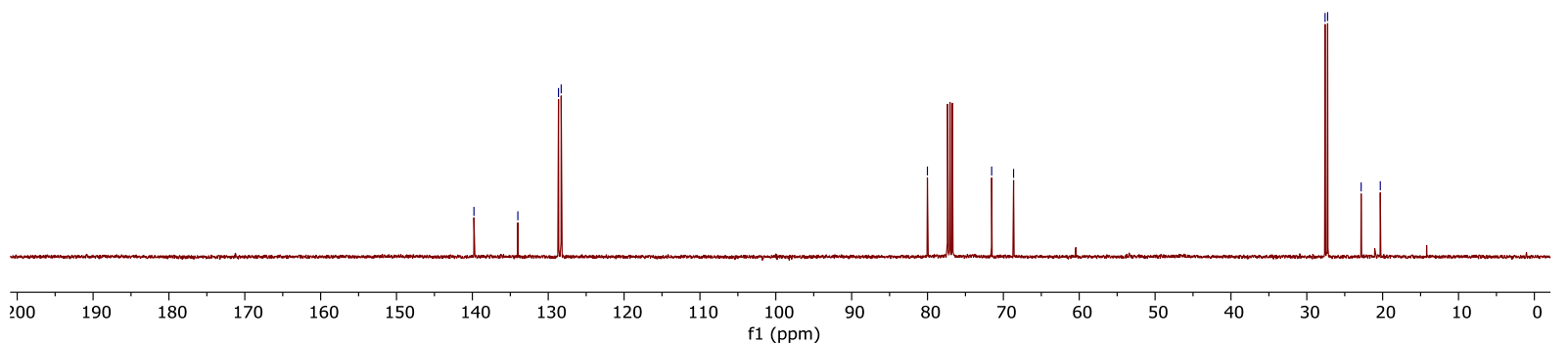


Compound $45\left({ }^{1} \mathrm{H}\right.$ NMR: $400 \mathrm{MHz},{ }^{13} \mathrm{C}\left\{{ }^{1} \mathrm{H}\right\}$ NMR: $\left.100 \mathrm{MHz}\right)$

ss-6-38-f29-33.1.fid

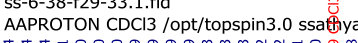

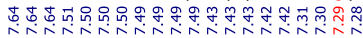

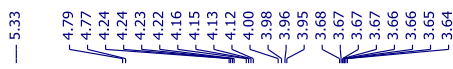

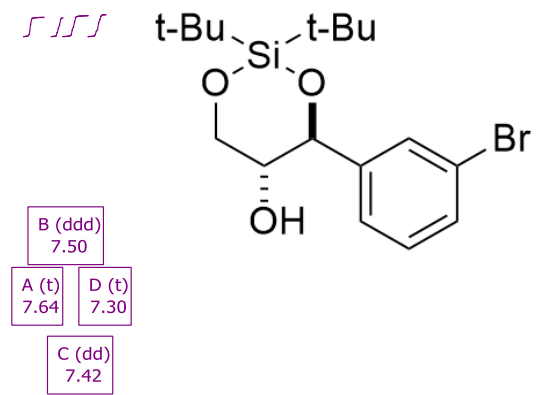
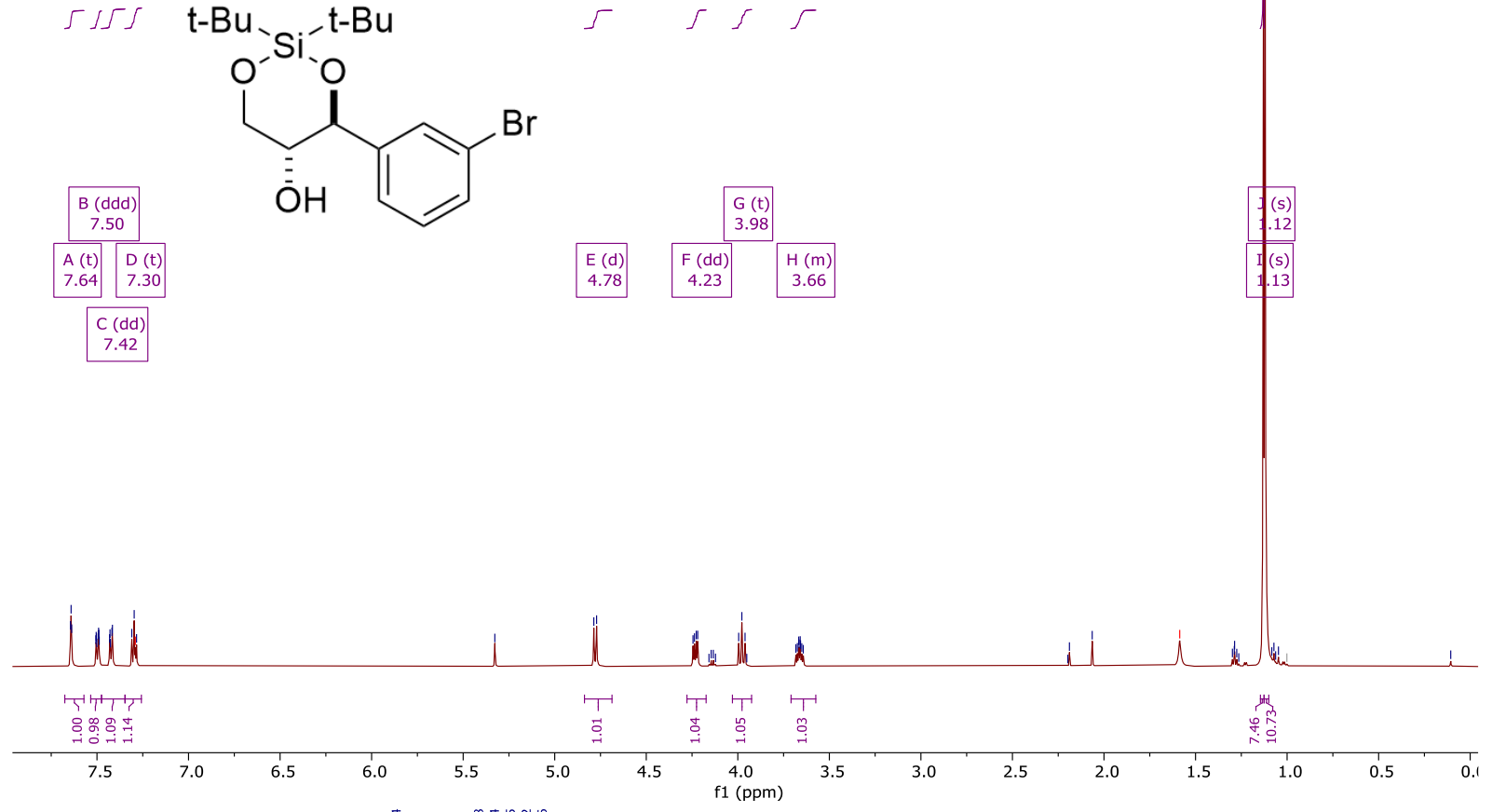

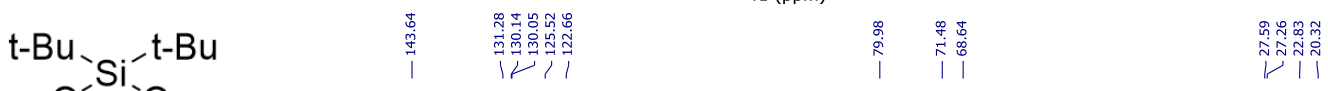<smiles>O[C@@H]1CO[SiH2]OC1c1cccc(Br)c1</smiles>

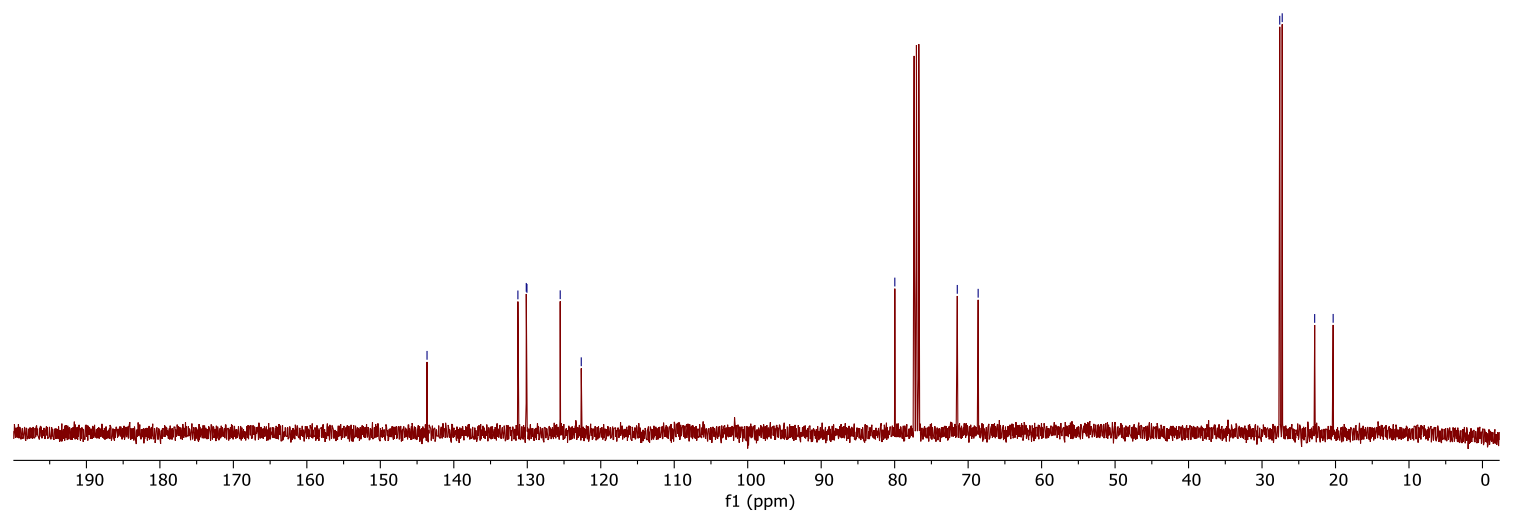




\section{Compound $46\left({ }^{1} \mathrm{H}\right.$ NMR: $400 \mathrm{MHz},{ }^{13} \mathrm{C}\left\{{ }^{1} \mathrm{H}\right\}$ NMR: $\left.100 \mathrm{MHz}\right)$}
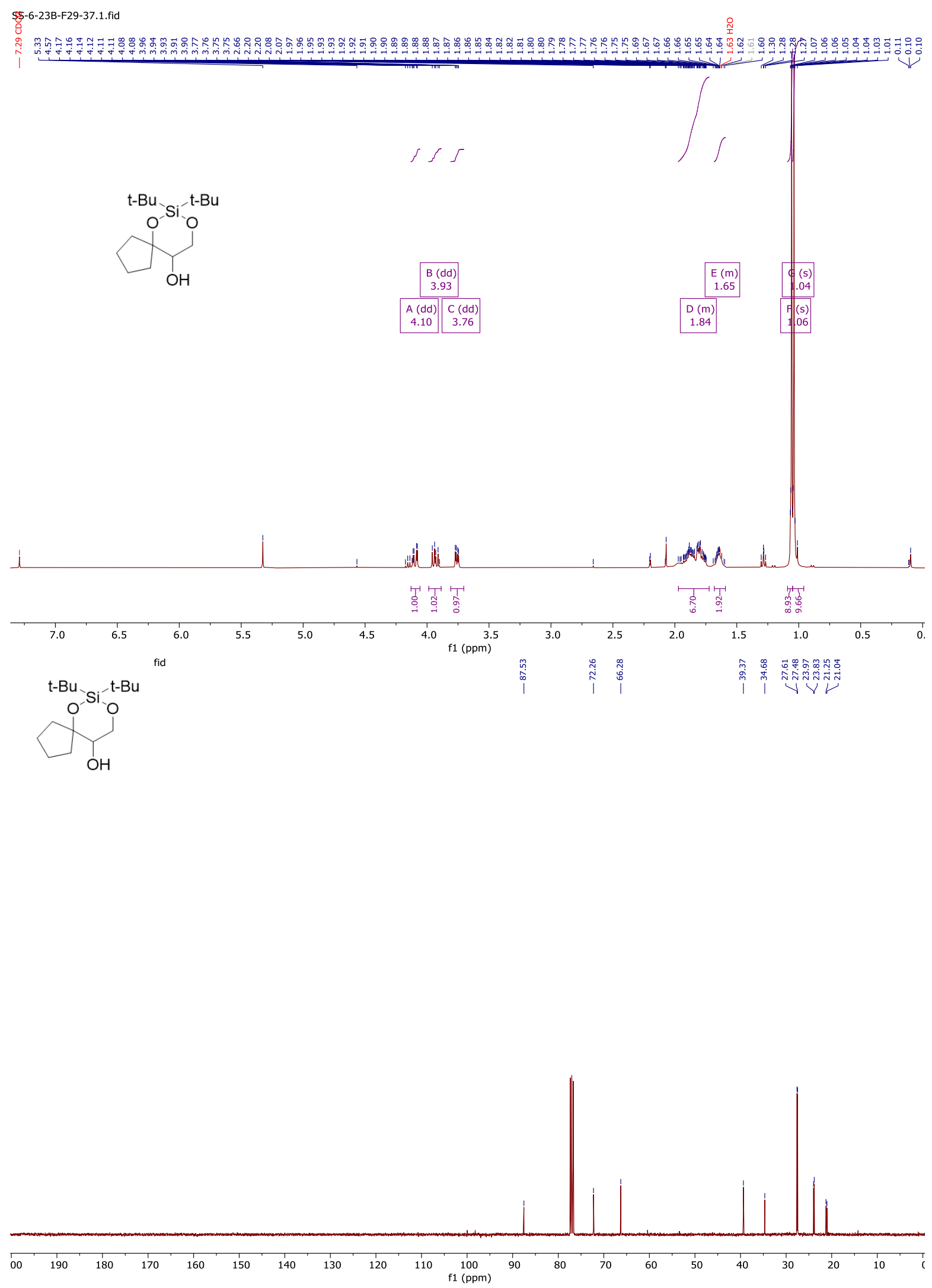
Compound $47\left({ }^{1} \mathrm{H}\right.$ NMR: $400 \mathrm{MHz},{ }^{13} \mathrm{C}\left\{{ }^{1} \mathrm{H}\right\}$ NMR: $100 \mathrm{MHz}$ )
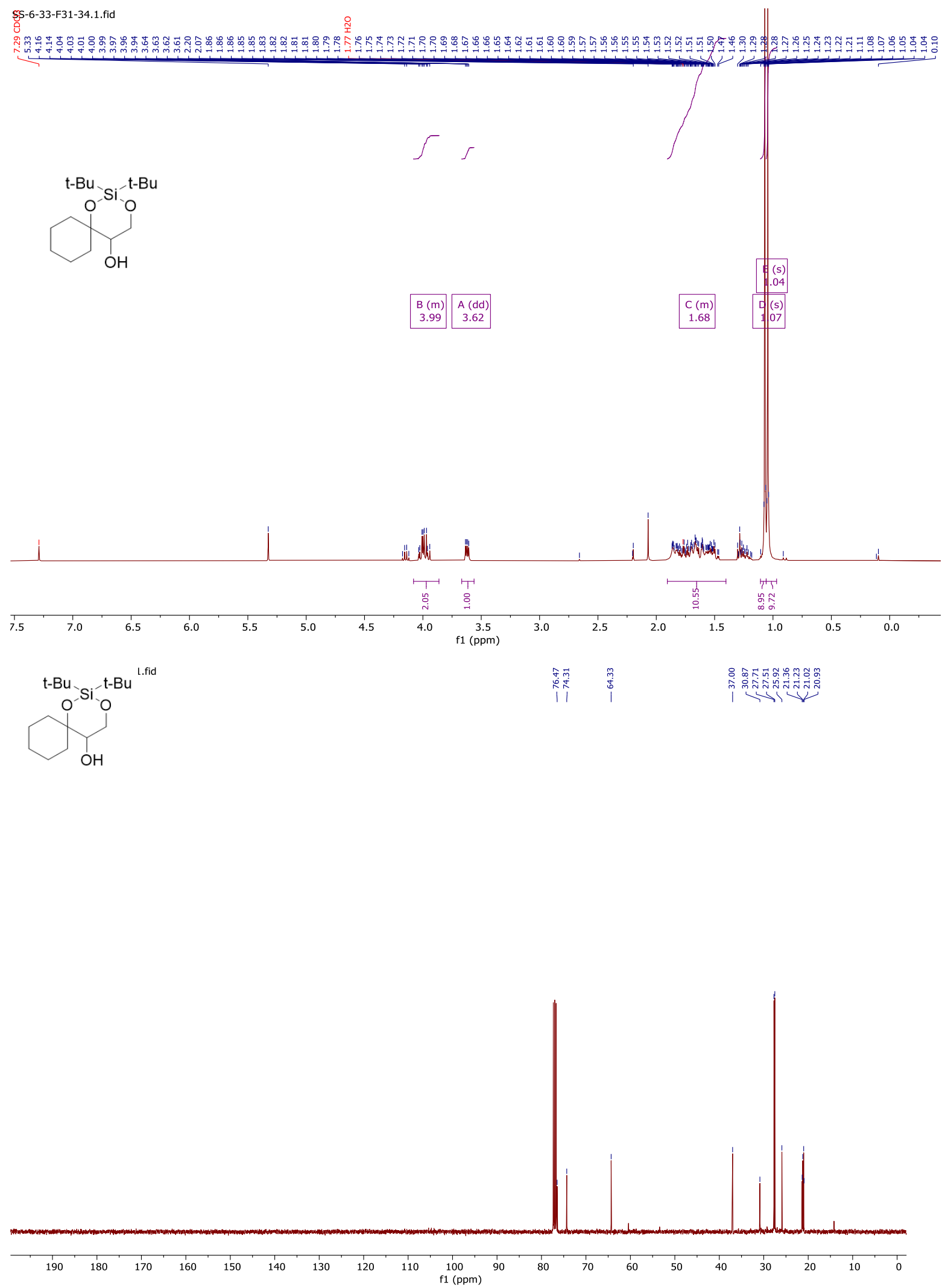
Compound $48\left({ }^{1} \mathrm{H}\right.$ NMR: $400 \mathrm{MHz},{ }^{13} \mathrm{C}\left\{{ }^{1} \mathrm{H}\right\}$ NMR: $\left.100 \mathrm{MHz}\right)$
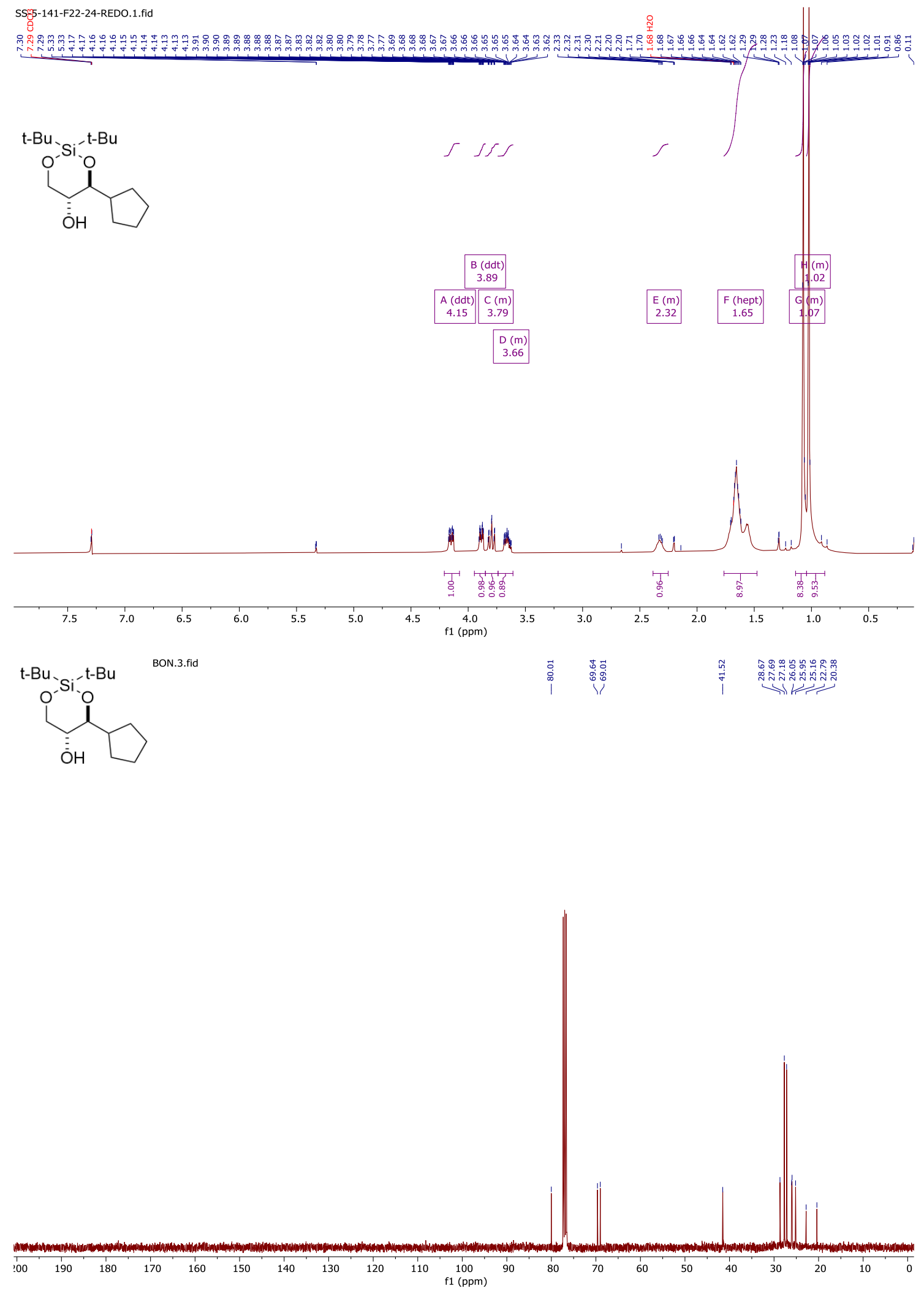
Compound 49 ( ${ }^{1} \mathrm{H}$ NMR: $400 \mathrm{MHz},{ }^{13} \mathrm{C}\left\{{ }^{1} \mathrm{H}\right\}$ NMR: $100 \mathrm{MHz}$ )

NS-I-g⿴囗十)

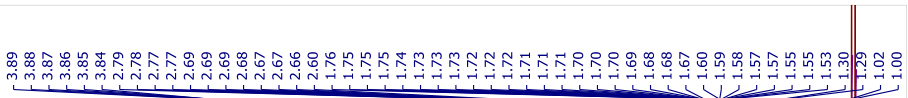

$\mathrm{Me}_{\mathrm{O}}^{\mathrm{t}-\mathrm{Bu}}$

$\iint S$

$\iiint$

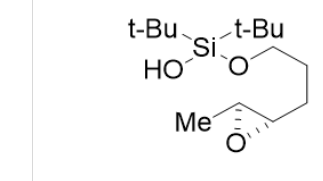

$(m)$

3.87

\begin{tabular}{|c|}
\hline $\begin{array}{c}\text { C (ddd) } \\
2.68\end{array}$ \\
\hline \begin{tabular}{c} 
B (dd) \\
2.78 \\
\hline$D(s)$ \\
2.60 \\
\hline
\end{tabular}
\end{tabular}

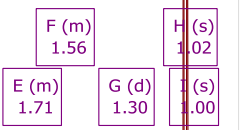

lal

N.

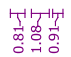

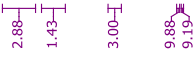

$\begin{array}{lllllllllllllllllllllllllllllllllllllllllllllllll}7.4 & 7.2 & 7.0 & 6.8 & 6.6 & 6.4 & 6.2 & 6.0 & 5.8 & 5.6 & 5.4 & 5.2 & 5.0 & 4.8 & 4.6 & 4.4 & 4.2 & 4.0 & 3.8 & 3.6 & 3.4 & 3.2 & 3.0 & 2.8 & 2.6 & 2.4 & 2.2 & 2.0 & 1.8 & 1.6 & 1.4 & 1.2 & 1.0 & 0 .\end{array}$

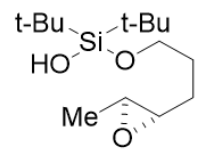

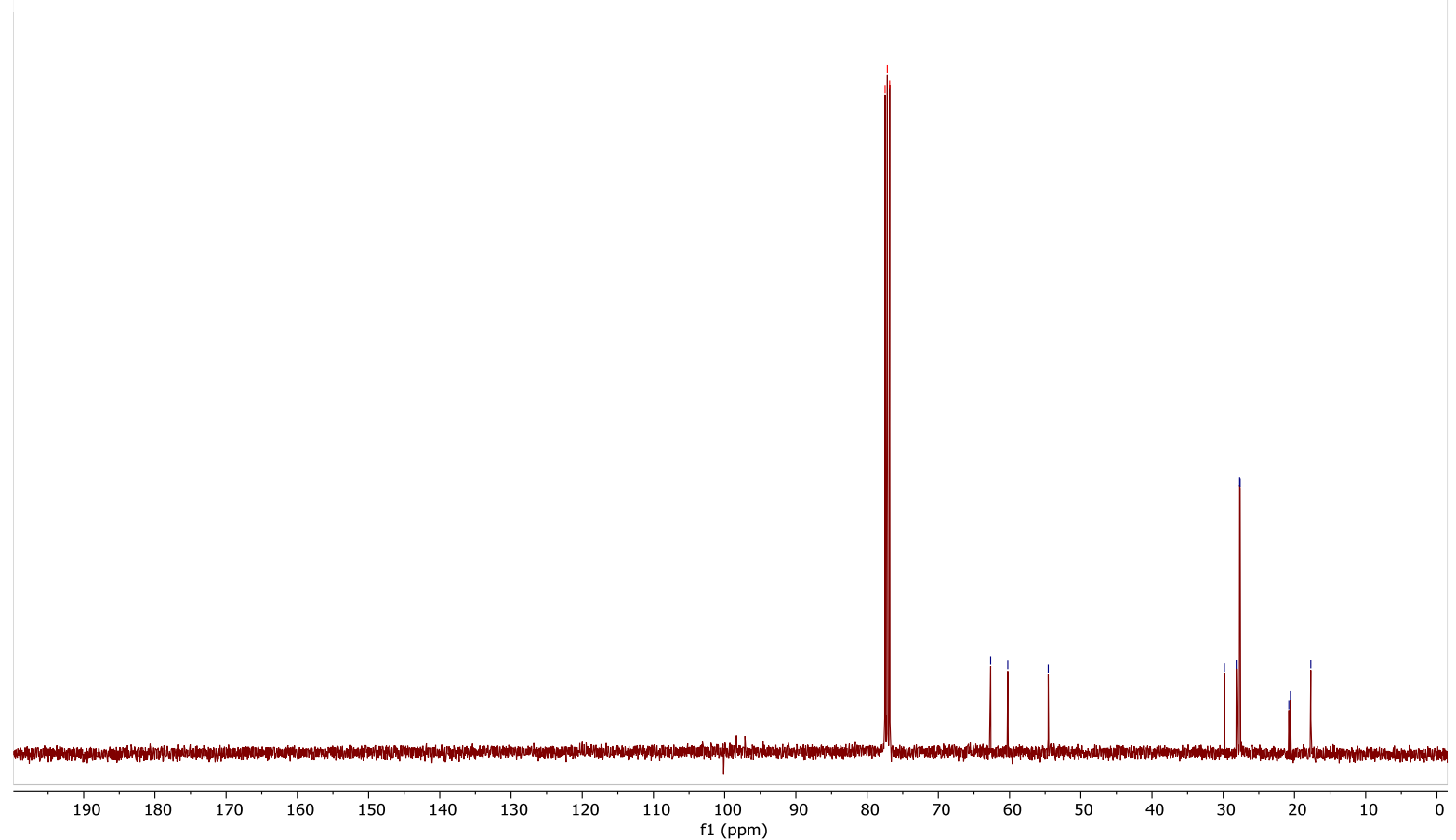


Compound 50 ( ${ }^{1} \mathrm{H}$ NMR: $400 \mathrm{MHz},{ }^{13} \mathrm{C}\left\{{ }^{1} \mathrm{H}\right\}$ NMR: $100 \mathrm{MHz}$ ) NS-I-g.g20.1.fid

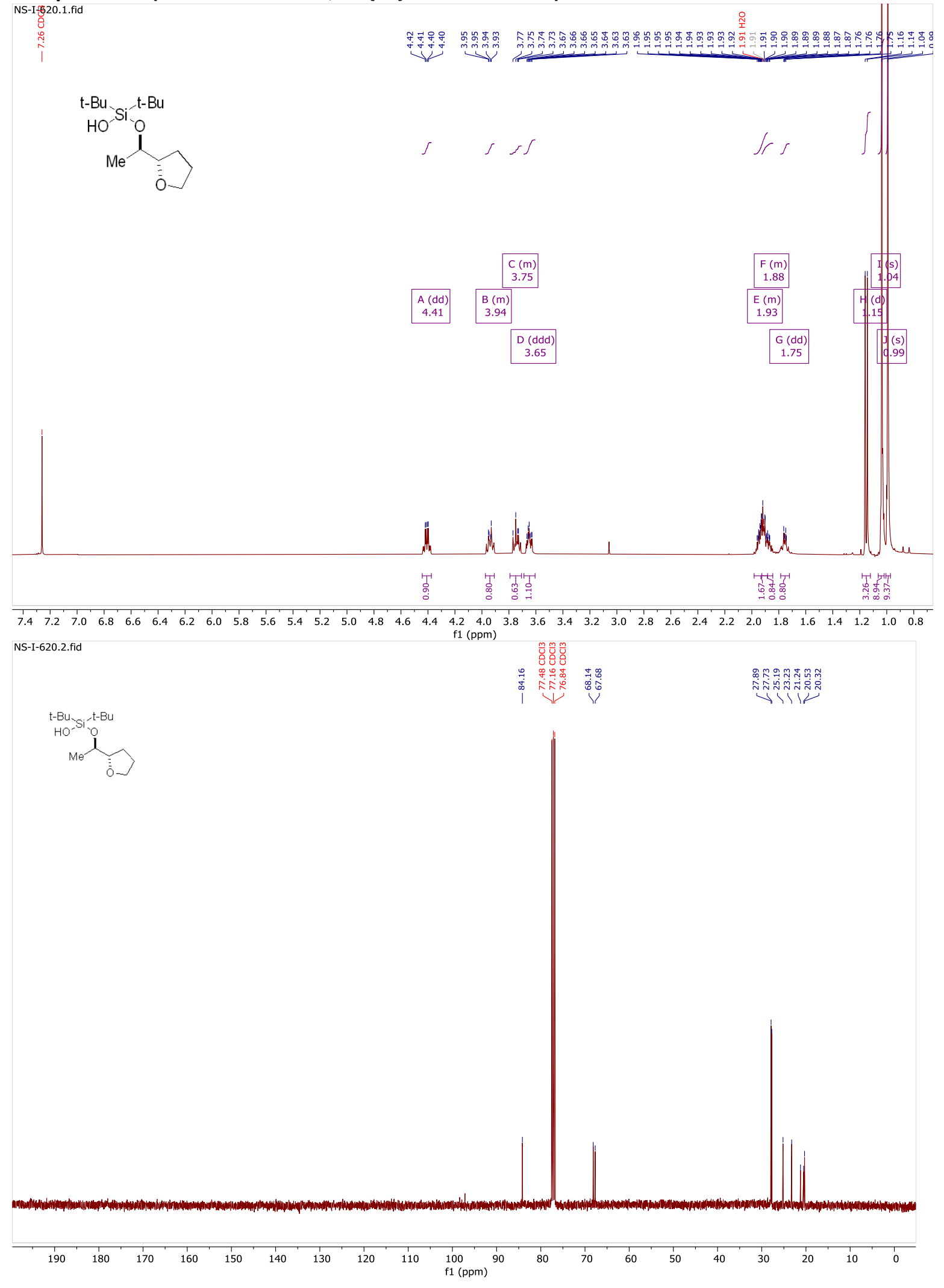


Compound $51\left({ }^{1} \mathrm{H}\right.$ NMR: $400 \mathrm{MHz},{ }^{13} \mathrm{C}\left\{{ }^{1} \mathrm{H}\right\}$ NMR: $\left.100 \mathrm{MHz}\right)$

NS-I-7æ?:1.fid

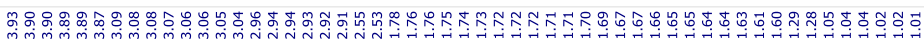

$\mathrm{t}^{\mathrm{Bu}}$

$\int$

S $S$

$\int$
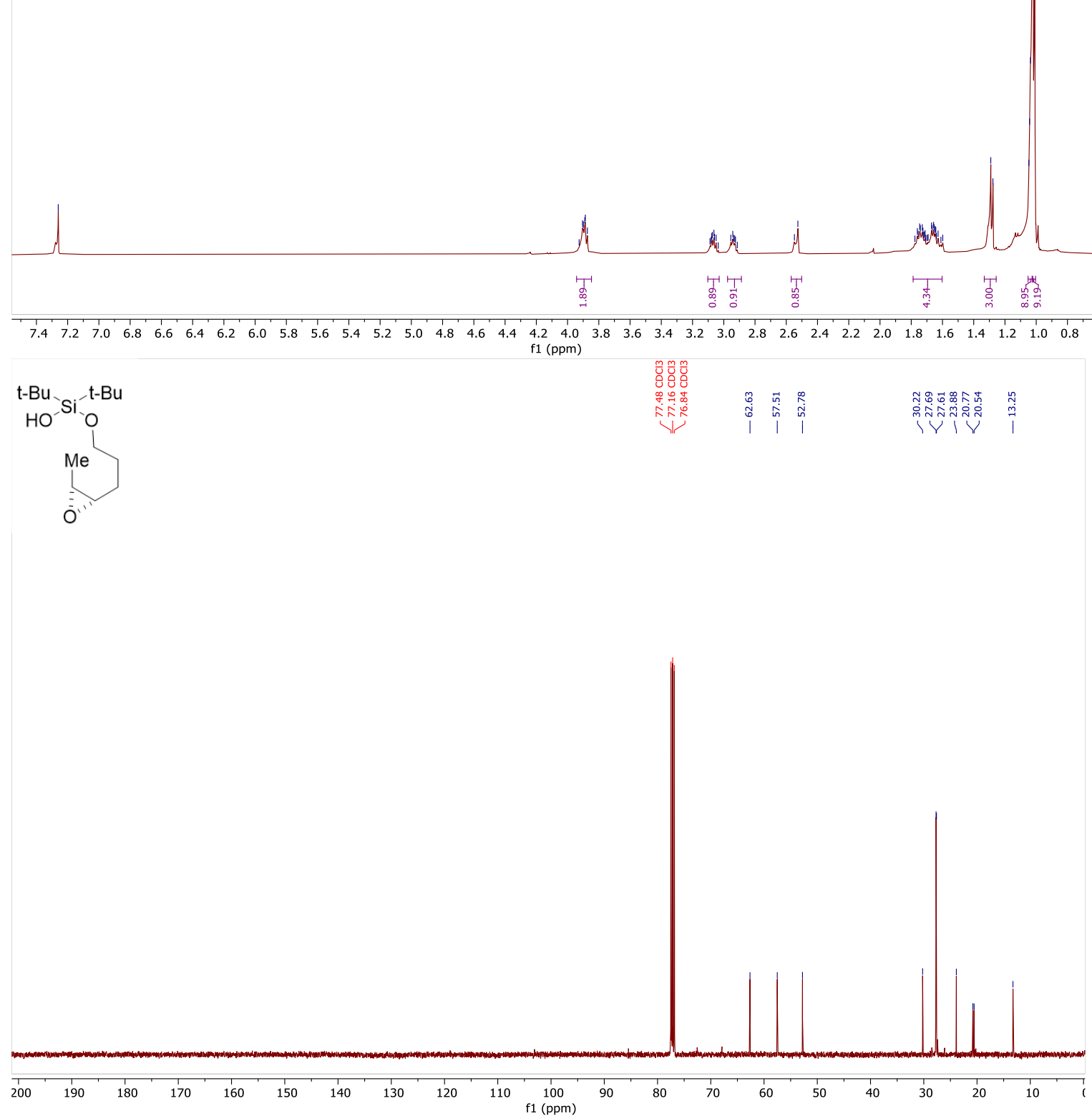
Compound $52\left({ }^{1} \mathrm{H}\right.$ NMR: $400 \mathrm{MHz},{ }^{13} \mathrm{C}\left\{{ }^{1} \mathrm{H}\right\}$ NMR: $\left.100 \mathrm{MHz}\right)$

NS-I-728.1.fid

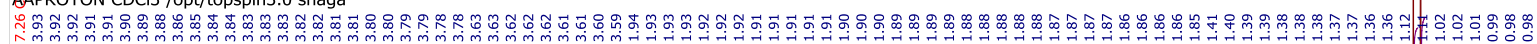
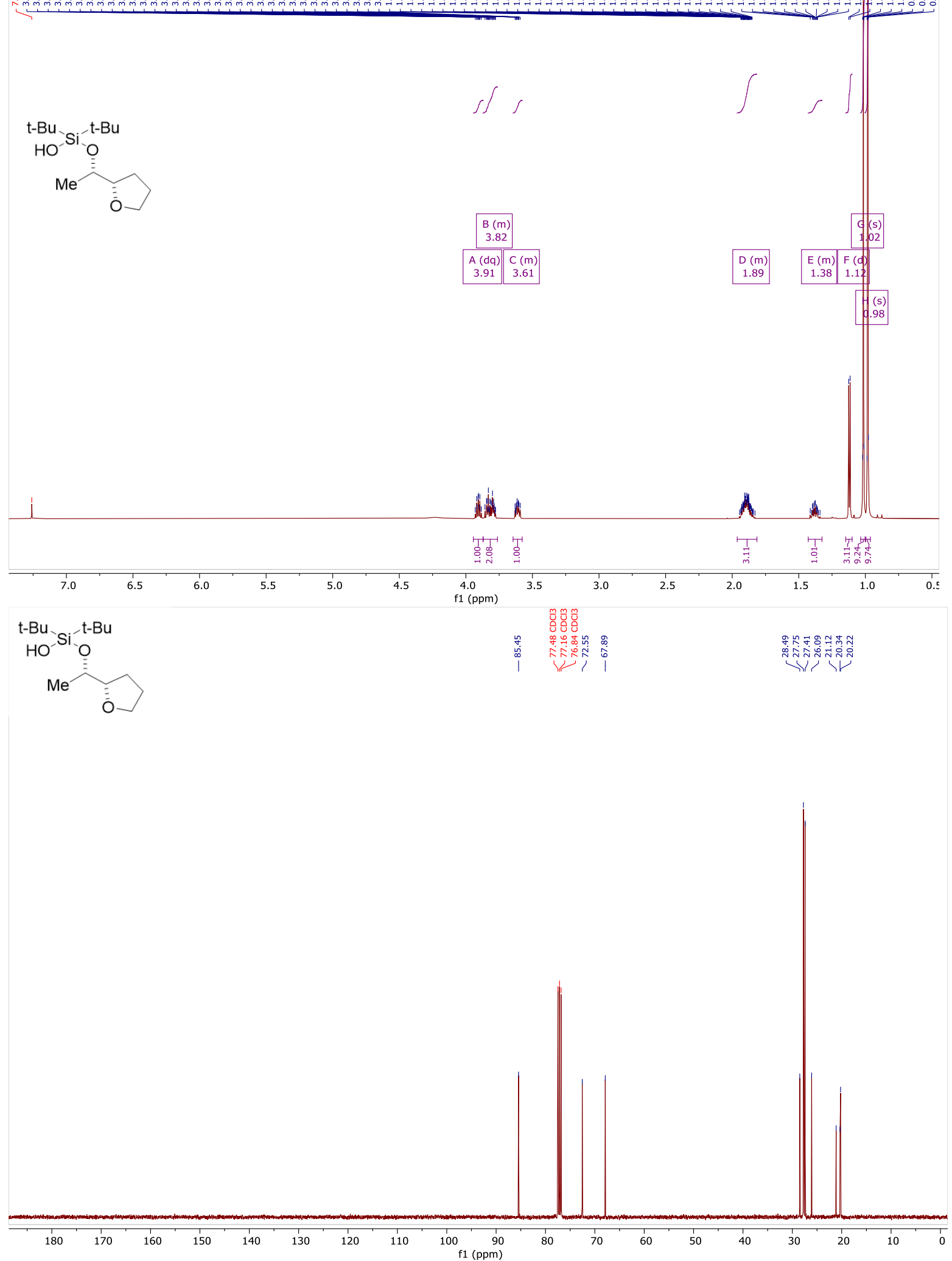
Compound $54\left({ }^{1} \mathrm{H}\right.$ NMR: $400 \mathrm{MHz},{ }^{13} \mathrm{C}\left\{{ }^{1} \mathrm{H}\right\}$ NMR: $\left.100 \mathrm{MHz}\right)$ NS-I-659.1.fid
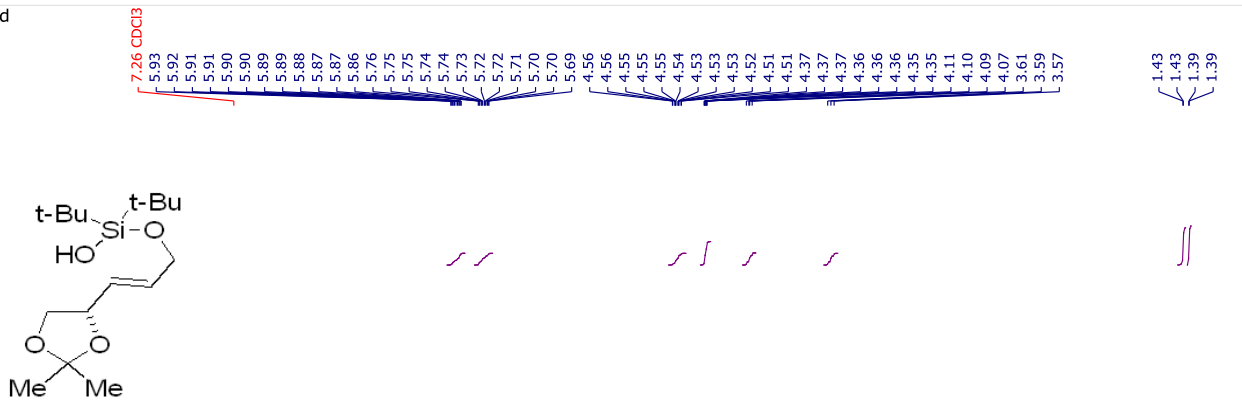

\begin{tabular}{|c|}
\hline $\begin{array}{c}\text { B (ddt) } \\
5.73\end{array}$ \\
\hline A (dtd) \\
5.89 \\
\hline
\end{tabular}
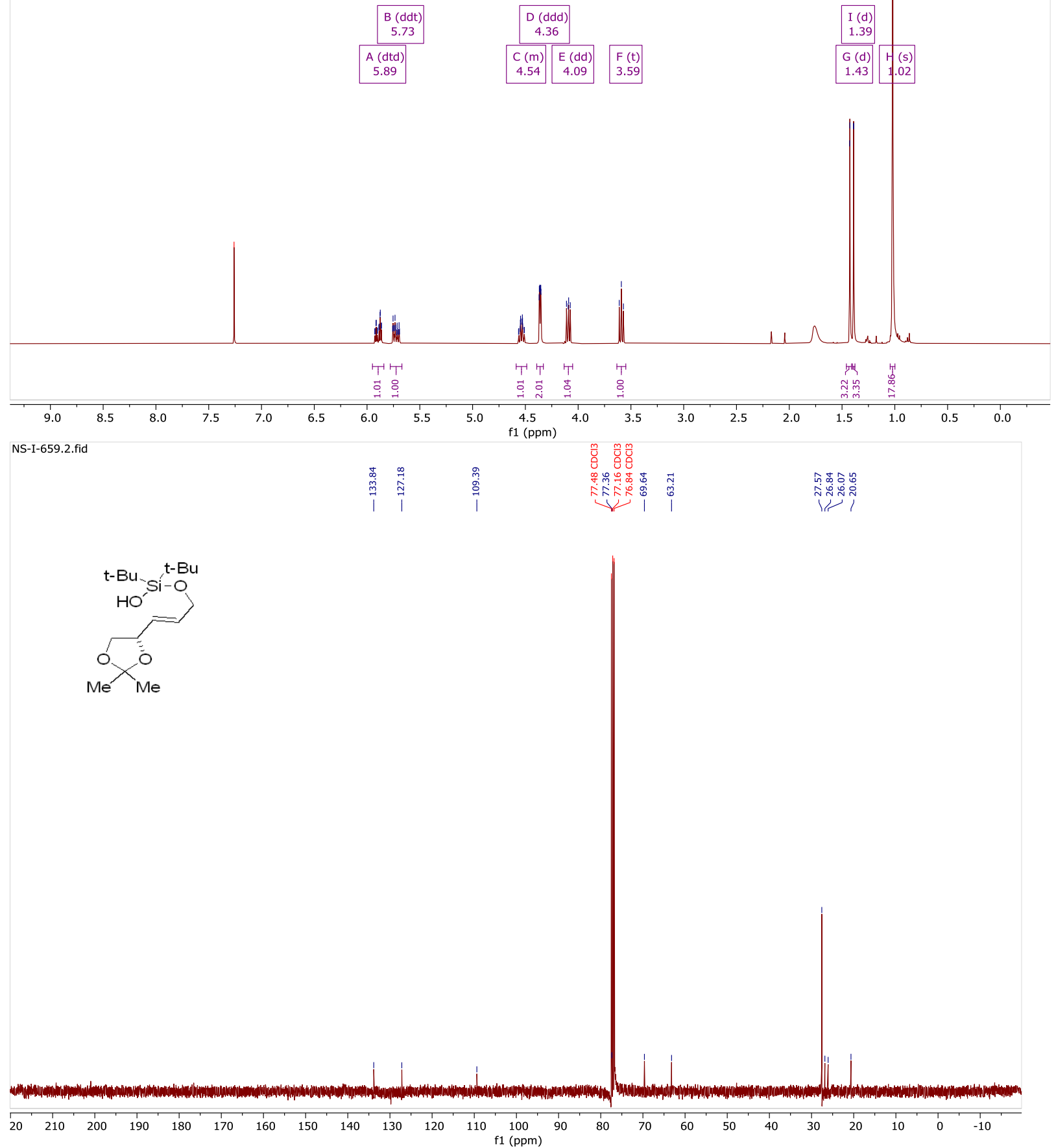
Compound $55\left({ }^{1} \mathrm{H}\right.$ NMR: $400 \mathrm{MHz},{ }^{13} \mathrm{C}\left\{{ }^{1} \mathrm{H}\right\}$ NMR: $\left.100 \mathrm{MHz}\right)$ ss-6-53-f36-41:

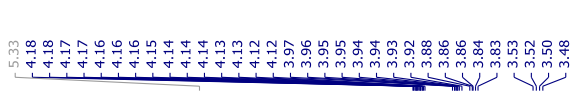
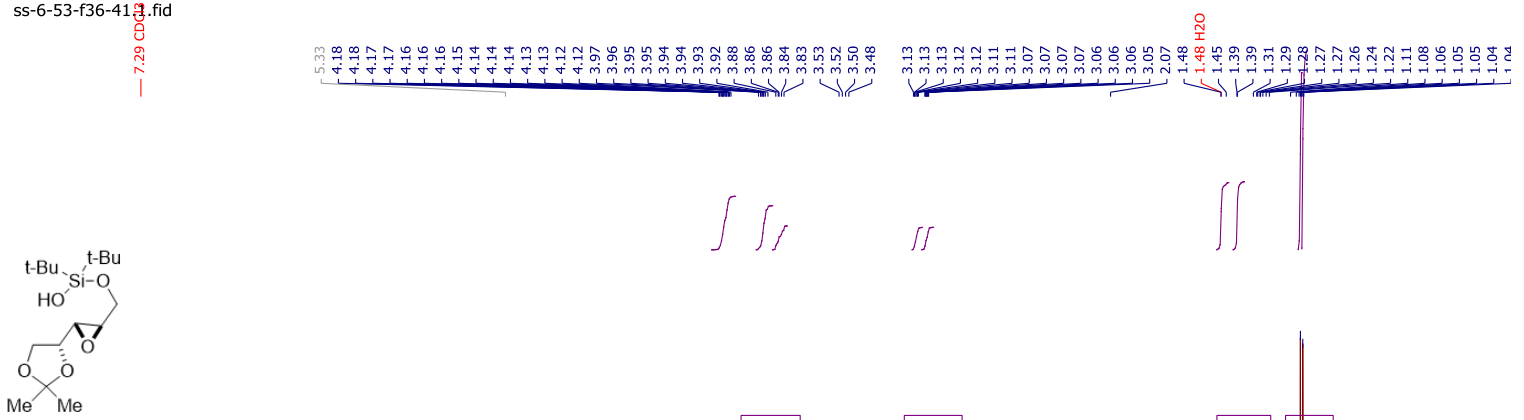

$\iint f$
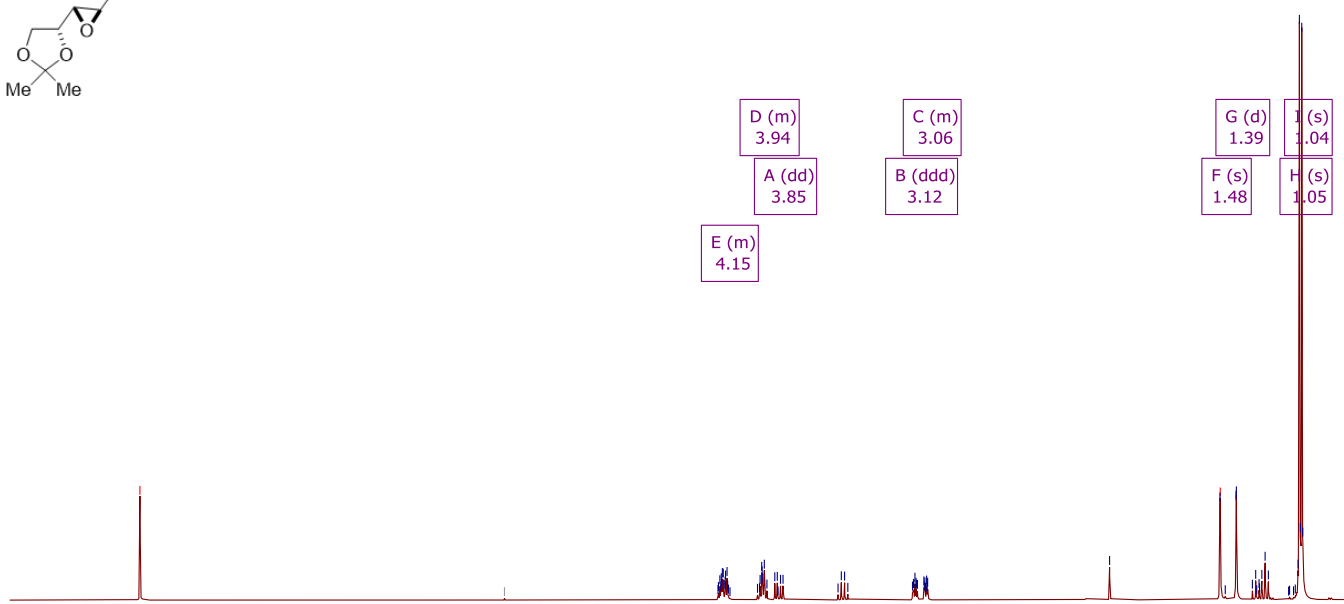

\begin{tabular}{|c|c|c|c|c|c|c|c|c|c|c|c|c|c|c|c|}
\hline & & & & & & & 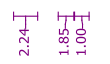 & & 整 & & & 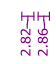 & 紅 & & \\
\hline 7.5 & 7.0 & 6.5 & $\begin{array}{l}1 \\
6.0\end{array}$ & 5.5 & 5.0 & 4.5 & $\begin{array}{c}4.0 \\
\text { f1 (ppm) }\end{array}$ & 3.5 & 3.0 & 2.5 & 2.0 & 1.5 & 1.0 & 0.5 & $\frac{T}{0.0}$ \\
\hline $\mathrm{t}-\mathrm{Bu} \cdot{ }_{-\mathrm{si}}^{\mathrm{t}-\mathrm{Bu}}$ & 1.fid & & & & & & 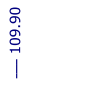 & & I & $\begin{array}{ll}0 & 0 \\
0 & 0 \\
0 & 0 \\
1 & 1\end{array}$ & $\begin{array}{l}\text { ․ำ } \\
\text { in } \\
11\end{array}$ & & & 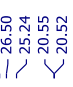 & \\
\hline
\end{tabular}

$\mathrm{HO}^{\prime}>$

O

$\mathrm{Me} \mathrm{Me}$

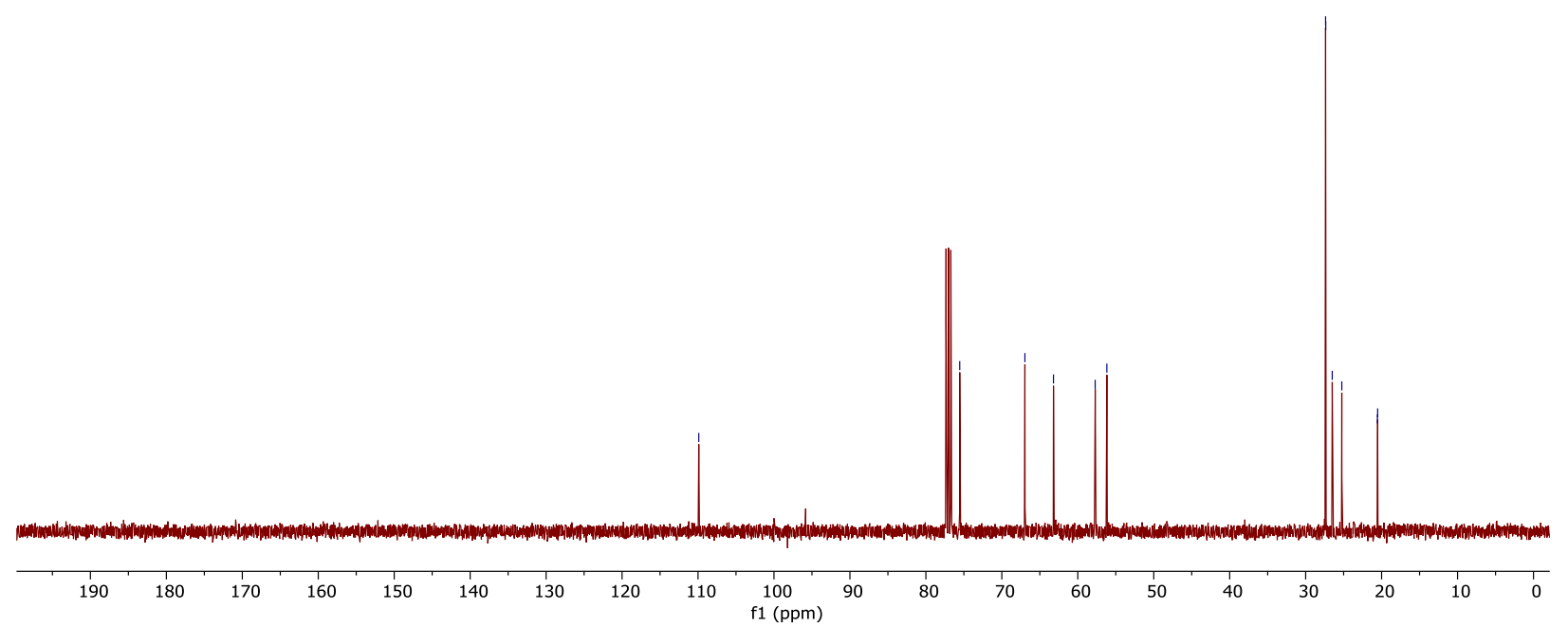


Compound $56\left({ }^{1} \mathrm{H}\right.$ NMR: $400 \mathrm{MHz},{ }^{13} \mathrm{C}\left\{{ }^{1} \mathrm{H}\right\}$ NMR: $\left.100 \mathrm{MHz}\right)$
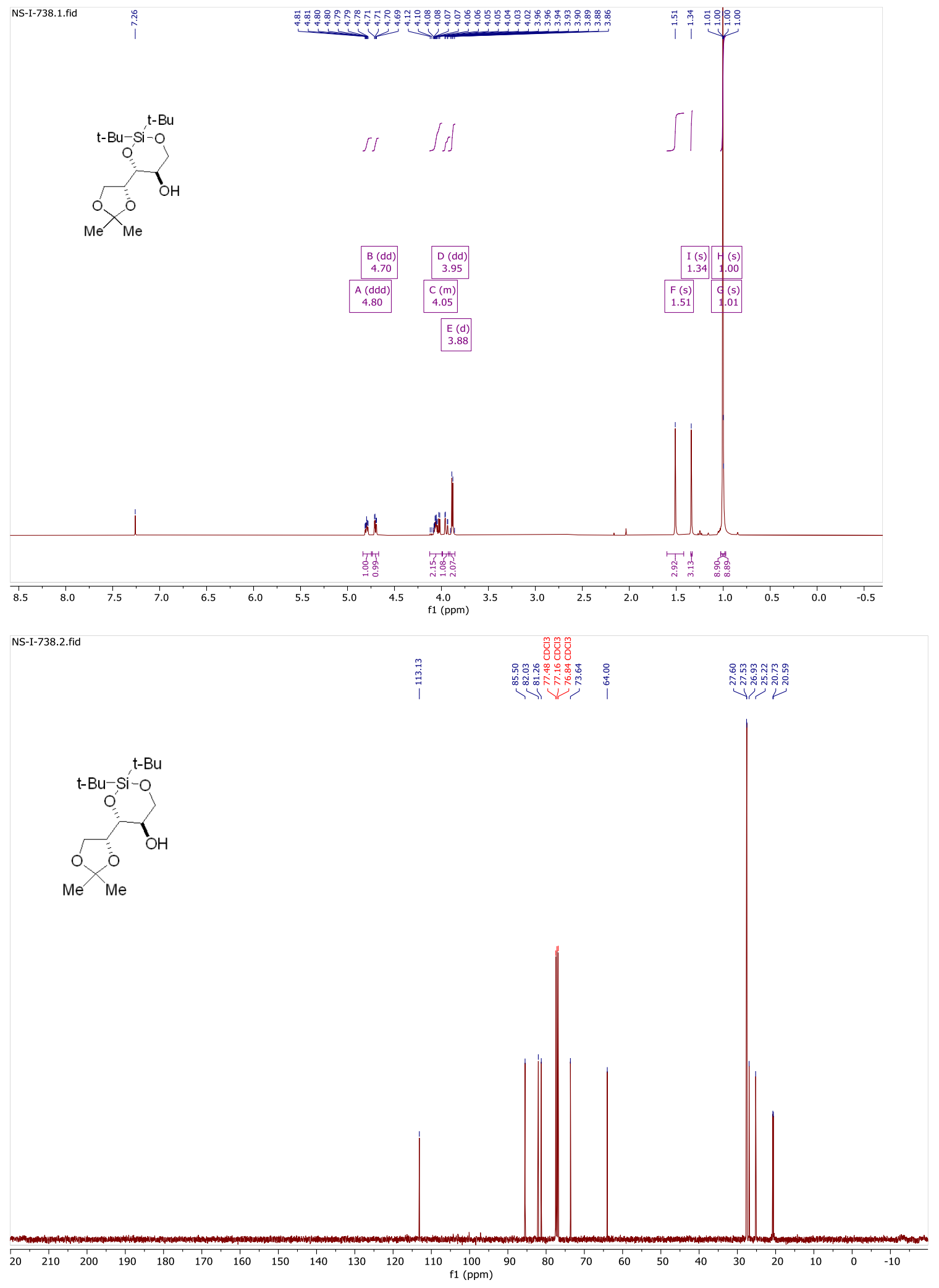
Compound 57 ( ${ }^{1} \mathrm{H}$ NMR: $400 \mathrm{MHz},{ }^{13} \mathrm{C}\left\{{ }^{1} \mathrm{H}\right\}$ NMR: $100 \mathrm{MHz}$ )
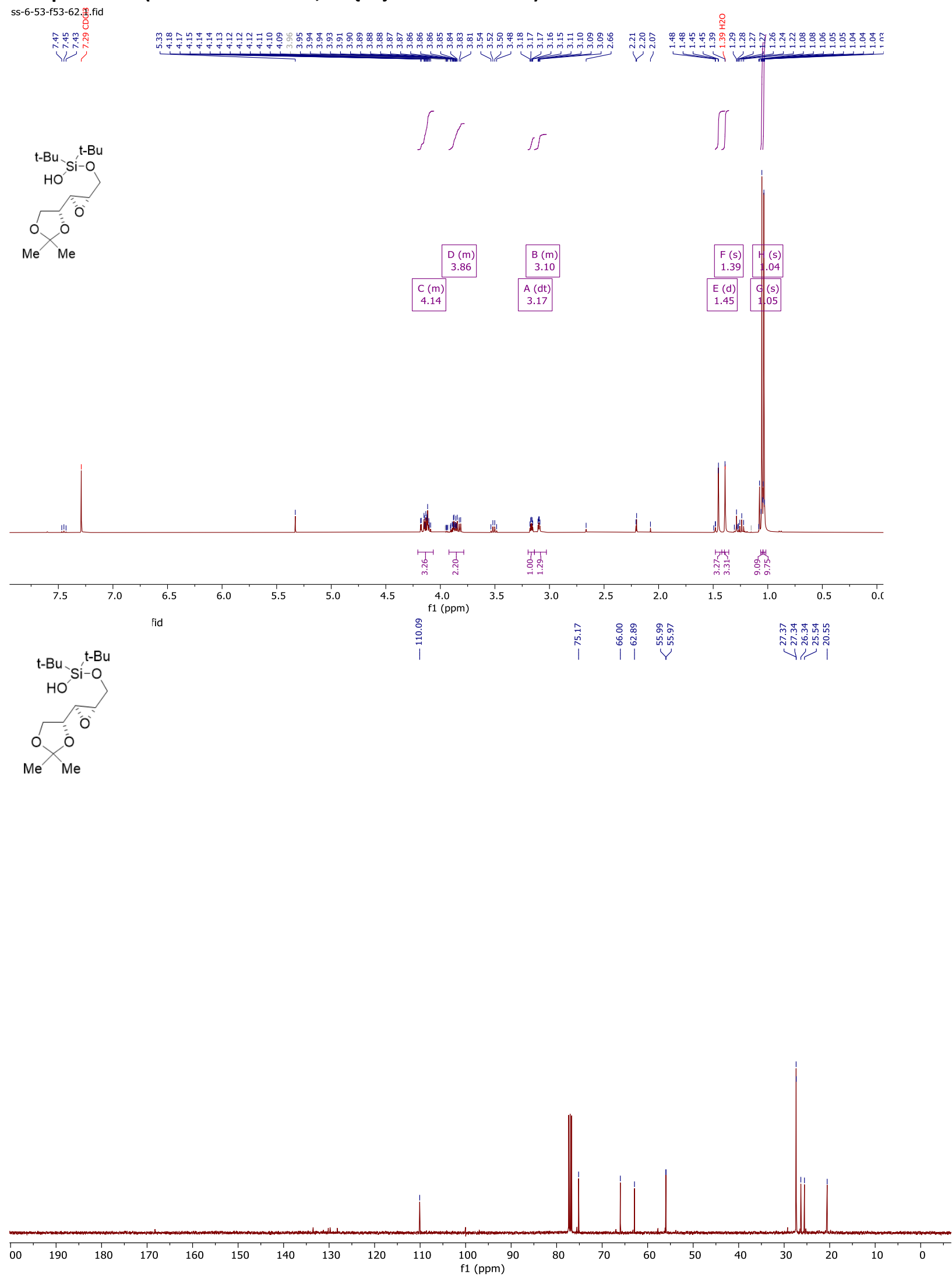
Compound $58\left({ }^{1} \mathrm{H}\right.$ NMR: $400 \mathrm{MHz},{ }^{13} \mathrm{C}\left\{{ }^{1} \mathrm{H}\right\}$ NMR: $\left.100 \mathrm{MHz}\right)$
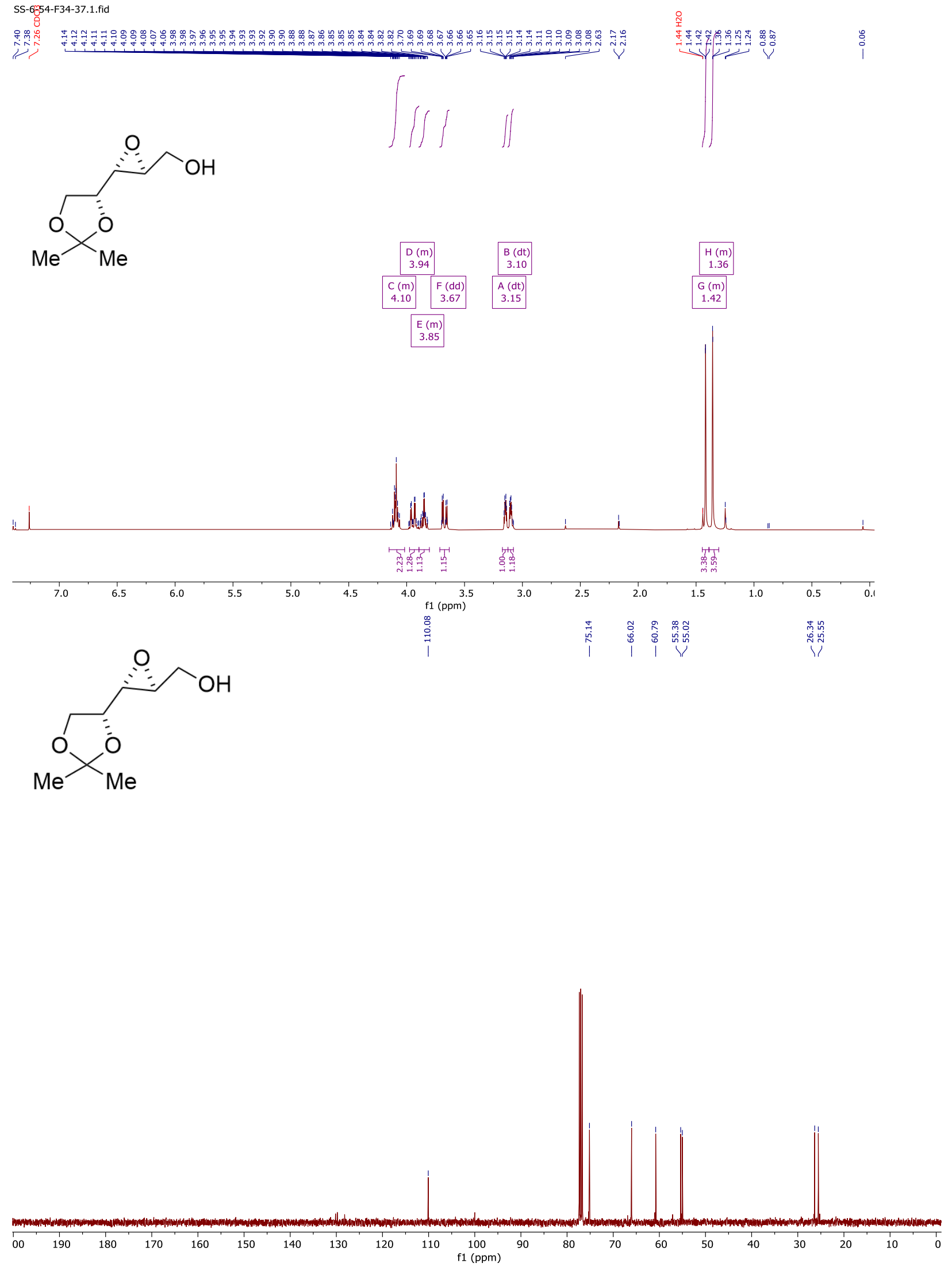